REPRESENTATION THEORY

An Electronic Journal of the American Mathematical Society

Volume 16, Pages 1-87 (January 11, 2012)

S $1088-4165(2012) 00406-X$

\title{
ON THE CLASSIFICATION OF IRREDUCIBLE REPRESENTATIONS OF AFFINE HECKE ALGEBRAS WITH UNEQUAL PARAMETERS
}

\author{
MAARTEN SOLLEVELD
}

\begin{abstract}
Let $\mathcal{R}$ be a root datum with affine Weyl group $W$, and let $\mathcal{H}=$ $\mathcal{H}(\mathcal{R}, q)$ be an affine Hecke algebra with positive, possibly unequal, parameters $q$. Then $\mathcal{H}$ is a deformation of the group algebra $\mathbb{C}[W]$, so it is natural to compare the representation theory of $\mathcal{H}$ and of $W$.

We define a map from irreducible $\mathcal{H}$-representations to $W$-representations and we show that, when extended to the Grothendieck groups of finite dimensional representations, this map becomes an isomorphism, modulo torsion. The map can be adjusted to a (nonnatural) continuous bijection from the dual space of $\mathcal{H}$ to that of $W$. We use this to prove the affine Hecke algebra version of a conjecture of Aubert, Baum and Plymen, which predicts a strong and explicit geometric similarity between the dual spaces of $\mathcal{H}$ and $W$.

An important role is played by the Schwartz completion $\mathcal{S}=\mathcal{S}(\mathcal{R}, q)$ of $\mathcal{H}$, an algebra whose representations are precisely the tempered $\mathcal{H}$-representations. We construct isomorphisms $\zeta_{\epsilon}: \mathcal{S}\left(\mathcal{R}, q^{\epsilon}\right) \rightarrow \mathcal{S}(\mathcal{R}, q)(\epsilon>0)$ and injection $\zeta_{0}: \mathcal{S}(W)=\mathcal{S}\left(\mathcal{R}, q^{0}\right) \rightarrow \mathcal{S}(\mathcal{R}, q)$, depending continuously on $\epsilon$.

Although $\zeta_{0}$ is not surjective, it behaves like an algebra isomorphism in many ways. Not only does $\zeta_{0}$ extend to a bijection on Grothendieck groups of finite dimensional representations, it also induces isomorphisms on topological $K$-theory and on periodic cyclic homology (the first two modulo torsion). This proves a conjecture of Higson and Plymen, which says that the $K$-theory of the $C^{*}$-completion of an affine Hecke algebra $\mathcal{H}(\mathcal{R}, q)$ does not depend on the parameter(s) $q$.
\end{abstract}

\section{INTRODUCTION}

Let $\mathcal{R}=\left(X, R_{0}, Y, R_{0}^{\vee}, F_{0}\right)$ be a based root datum with finite Weyl group $W_{0}$ and (extended) affine Weyl group $W=W_{0} \ltimes X$. For every parameter function $q: W \rightarrow \mathbb{C}^{\times}$there is an affine Hecke algebra $\mathcal{H}=\mathcal{H}(\mathcal{R}, q)$.

The most important and most studied case is when $q$ takes the same value on all simple (affine) reflections in $W$. If this value is a prime power, then $\mathcal{H}$ is isomorphic to the convolution algebra of Iwahori-bi-invariant functions on a reductive $p$-adic group with root datum $\mathcal{R}^{\vee}=\left(Y, R_{0}^{\vee}, X, R_{0}, F_{0}^{\vee}\right)$ IwMa. Suppose that the complex Lie group $G_{\mathbb{C}}$ with root datum $\mathcal{R}$ has a simply connected derived group. Then the generic Hecke algebra obtained by replacing $q$ with a formal variable $\mathbf{q}$ is known $\left[\mathrm{KaLu1}, \mathrm{ChGi}\right.$ to be isomorphic to the $G_{\mathbb{C}} \times \mathbb{C}^{\times}$-equivariant $K$-theory of the Steinberg variety of $G_{\mathbb{C}}$. Via this geometric interpretation Kazhdan and Lusztig KaLu2 classified and constructed all irreducible representations of $\mathcal{H}(\mathcal{R}, q)$ (when $q \in \mathbb{C}^{\times}$is not a root of unity), in accordance with the local Langlands program.

Received by the editors September 27, 2010 and, in revised form, May 31, 2011.

2010 Mathematics Subject Classification. Primary 20C08; Secondary 20 G25.

(C) 2012 American Mathematical Society 
On the other hand, the definition of affine Hecke algebras with generators and relations allows one to choose the values of $q$ on non-conjugate simple reflections independently. Although this might appear to be an innocent generalization, much less is known about affine Hecke algebras with unequal parameters. The reason is that Lusztig's constructions in equivariant $K$-theory KaLu1 allow only one deformation parameter. Kato Kat2 invented a more complicated variety, called an exotic nilpotent cone, which plays a similar role for the three parameter affine Hecke algebra of type $C_{n}^{(1)}$. From this, one can extract a classification of the tempered dual, for an arbitrary parameter function $q$ CiKa.

Like equal parameter affine Hecke algebras, those with unequal parameters also arise as intertwining algebras in the smooth representation theory of reductive $p$ adic groups. One can encounter them if one looks at nonsupercuspidal Bernstein components (in the smooth dual) Lus7, Mor. Even for split groups unequal parameters occur, albeit not in the principal series [Roc1]. It is expected that every Bernstein component of a $p$-adic group can be described with an affine Hecke algebra or a slight variation on it. In [BaMo1, BaMo2, $\mathrm{BaCi}$ ] it is shown, in increasing generality, that under certain conditions the equivalence between the module category of an affine Hecke algebra and a Bernstein block (in the category of smooth modules) respects unitarity. Thus affine Hecke algebras are an important tool for the classification of both the smooth dual and the unitary smooth dual of a reductive $p$-adic group.

The degenerate versions of affine Hecke algebras are usually called graded Hecke algebras. Their role in the representation theory of reductive $p$-adic groups [Lus6, Lus7, BaMo2, $\mathrm{Ciu}$, is related to affine Hecke algebras in the way that Lie algebras stand to Lie groups. They have a very simple presentation, which makes is possible to let them act on many function spaces. Therefore one encounters graded Hecke algebras (with possibly unequal parameters) also independently from affine Hecke algebras, for instance, in certain systems of differential equations $\mathrm{HeOp}$ and in the study of the unitary dual of a real reductive group $\mathrm{CiTr}$.

In view of the above connections, it is of considerable interest to classify the dual of an affine or graded Hecke algebra with unequal parameters. Since $\mathcal{H}(\mathcal{R}, q)$ is a deformation of the group algebra $\mathbb{C}[W]$, it is natural to expect strong similarities between the duals $\operatorname{Irr}(\mathcal{H}(\mathcal{R}, q))$ and $\operatorname{Irr}(W)$. Indeed, for equal parameters the Deligne-Langlands-Kazhdan-Lusztig parametrization provides a bijection between these duals [KaLu2, Lus5]. For unequal parameters we approach the issue via harmonic analysis on affine Hecke algebras, which forces us to consider only parameter functions $q$ with values in $\mathbb{R}_{>0}$. We will assign to every irreducible $\mathcal{H}$-representation $\pi$ in a natural way a representation $\zeta^{*}(\pi)$ of the extended affine Weyl group $W$. Although this construction does not always preserve irreducibility, it has many nice properties, of which the most important is:

Theorem 1 (see Theorem 2.3.1). The collection of representations $\left\{\zeta^{*}(\pi): \pi \in\right.$ $\operatorname{Irr}(\mathcal{H}(\mathcal{R}, q))\}$ forms a $\mathbb{Q}$-basis of the representation ring of $W$.

Since the Springer correspondence for finite Weyl groups realizes $\operatorname{Irr}\left(W_{0}\right)$, via Kazhdan-Lusztig theory, as a specific subset of $\operatorname{Irr}(\mathcal{H}(\mathcal{R}, q))$, Theorem 1 can be regarded as an affine Springer correspondence without irreducibility. By picking suitable irreducible subquotients of the $\zeta^{*}(\pi)$ one can refine Theorem 1 to a bijection $\operatorname{Irr}(\mathcal{H}(\mathcal{R}, q)) \rightarrow \operatorname{Irr}(W)$. This is related to a conjecture of Aubert, Baum and Plymen ABP1, ABP2 (ABP-conjecture for short) which we sketch here. 
Recall that $W=W_{0} \ltimes X$ and let $T$ be the complex torus $\operatorname{Hom}_{\mathbb{Z}}\left(X, \mathbb{C}^{\times}\right)$. Clifford theory says that the irreducible $W$-representations with an $X$-weight $t \in T$ are in natural bijection with the irreducible representations of the isotropy group $W_{0, t}$. Write $T^{w}=\{t \in T: w(t)=t\}$. The extended quotient of $T$ by $W_{0}$ is defined as

$$
\widetilde{T} / W_{0}=\bigsqcup_{w \in W_{0}}\{w\} \times T^{w} / W_{0},
$$

with respect to the $W_{0}$-action $w \cdot\left(w^{\prime}, t\right)=\left(w w^{\prime} w^{-1}, w t\right)$. We endow $\widetilde{T} / W_{0}$ with the topology coming from the discrete topology on $W_{0}$ and the Zariski topology on $T$. It is a model for $\operatorname{Irr}(W)$ with the Jacobson topology, in the sense that there exist continuous bijections $\widetilde{T} / W_{0} \rightarrow \operatorname{Irr}(W)$ which respect the projections to $T / W_{0}$.

The Bernstein presentation (included as Theorem 1.2.1) shows that $\mathbb{C}[X] \cong \mathcal{O}(T)$ is naturally isomorphic to a commutative subalgebra $\mathcal{A} \subset \mathcal{H}(\mathcal{R}, q)$, and that the center of $\mathcal{H}(\mathcal{R}, q)$ is $\mathcal{A}^{W_{0}} \cong \mathcal{O}\left(T / W_{0}\right)$. Hence we have a natural map $\operatorname{Irr}(\mathcal{H}(\mathcal{R}, q)) \rightarrow$ $T / W_{0}$, sending a representation to its central character. It is continuous with respect to the Jacobson topology on $\operatorname{Irr}(\mathcal{H}(\mathcal{R}, q))$. A simplified version of the ABPconjecture for affine Hecke algebras reads:

Theorem 2 (see Theorem 5.4.2). Let $\mathcal{H}(\mathcal{R}, q)$ be an affine Hecke algebra with positive, possibly unequal, parameters. Let $\mathcal{Q}(\mathcal{R})$ be the variety of parameter functions $W \rightarrow \mathbb{C}^{\times}$, endowed with the Zariski topology. There exist a continuous bijection $\mu: \widetilde{T} / W_{0} \rightarrow \operatorname{Irr}(\mathcal{H}(\mathcal{R}, q))$ and a map $h: \widetilde{T} / W_{0} \times \mathcal{Q}(\mathcal{R}) \rightarrow T$ such that:

- $h$ is locally constant in the first argument;

- for fixed $c \in \widetilde{T} / W_{0}, h(c, v)$ is a monomial in the variables $v(s)^{ \pm 1}$, where $s$ runs through all simple affine reflections;

- the central character of $\mu\left(W_{0}(w, t)\right)$ is $W_{0} h\left(W_{0}(w, t), q^{1 / 2}\right) t$.

The author hopes that Theorem 2 will be useful in the local Langlands program. This could be the case for a Bernstein component $\mathfrak{s}$ of a reductive $p$-adic group $G$ for which $\operatorname{Mod}_{\mathfrak{s}}(G)$ is equivalent to $\operatorname{Mod}(\mathcal{H}(\mathcal{R}, q)$ ) (see Section 1.6 for the notation). Recall that a Langlands parameter for $G$ is a certain kind of group homomorphism $\phi: \mathcal{W}_{\mathbb{F}} \ltimes \mathbb{C} \rightarrow{ }^{L} G$, where ${ }^{L} G$ is the Langlands dual group and $\mathcal{W}_{\mathbb{F}}$ is the Weil group of the local field $\mathbb{F}$ over which $G$ is a variety. Let us try to extract a Langlands parameter from $W_{0}(w, t) \in \widetilde{T} / W_{0}$. The image of the distinguished Frobenius element of $\mathcal{W}_{\mathbb{F}}$ describes the central character, so modulo conjugacy it should be $h\left(W_{0}(w, t), q^{1 / 2}\right) t \in T \subset{ }^{L} G$.

Problematic is that the map $\mu$ from Theorem 2 is not canonical, its construction involves some arbitrary choices, which could lead to a different $w \in W_{0}$. Yet it is precisely this element $w$ that should determine the unipotent conjugacy class that contains the image of $\mathbb{C} \backslash\{0\}$ under $\phi$. Recently, Lusztig [Lus9] defined a map from conjugacy classes in a Weyl group to unipotent classes in the associated complex Lie group. Whether this map yields a suitable unipotent class for our hypothetical $\phi$ remains to be seen, for this it is probably necessary to find a more canonical construction of $\mu$. The rest of such a Langlands parameter $\phi$ is completely beyond affine Hecke algebras, it will have to depend on number theoretic properties of the Bernstein component $\mathfrak{s}$.

Now we describe the most relevant results needed for Theorems 10 and 2 A large step towards the determination of $\operatorname{Irr}(\mathcal{H}(\mathcal{R}, q))$ is the Langlands classification (see Theorem 2.2.4), which reduces the problem to the tempered duals of parabolic subalgebras of $\mathcal{H}(\mathcal{R}, q)$. This is of course a well-known result, for graded Hecke 
algebras due to Evens [Eve, but the proof for affine Hecke algebras has not been published before.

In line with Harish-Chandra's results for reductive groups, every tempered irreducible $\mathcal{H}(\mathcal{R}, q)$-representation appears as a direct summand of a representation that is induced from a discrete series representation of a parabolic subalgebra, a point of view advocated by Opdam Opd2. We note that in this setting tempered and discrete series representations can be defined very easily via the $\mathcal{A}$-weights of a representation. For affine Hecke algebras with irreducible root data, Opdam and the author classified the discrete series in OpSo2, but we emphasize that we do not use that classification in the present paper.

To every set of simple roots $P \subset F_{0}$ we associate a root subsystem $R_{P} \subset R_{0}$ and a parabolic subalgebra $\mathcal{H}_{P} \subset \mathcal{H}$, which is generated by $\mathcal{H}\left(W\left(R_{P}\right), q\right)$ and by a commutative subalgebra $\mathcal{A}_{P} \cong \mathbb{C}[X / X \cap \mathbb{Q} P]$. Induction from $\mathcal{H}_{P}$ allows an induction parameter in $T^{P}$, the subtorus of $T$ orthogonal to $P^{\vee}$. So we consider induction data $\xi=(P, \delta, t)$ where $P \subset F_{0}, t \in T^{P}$ and $\delta$ is a discrete series representation of $\mathcal{H}_{P}$. Such a triple gives rise to a parabolically induced representation

$$
\pi(\xi)=\pi(P, \delta, t)=\operatorname{Ind}_{\mathcal{H}^{P}}^{\mathcal{H}}\left(\delta \circ \phi_{t}\right) .
$$

Here $\mathcal{H}^{P} \subset \mathcal{H}$ is more or less a central extension of $\mathcal{H}_{P}$ by $\mathcal{O}\left(T^{P}\right)$ and $\phi_{t}: \mathcal{H}^{P} \rightarrow$ $\mathcal{H}_{P}$ is a twisted projection. The representation $\pi(\xi)$ is tempered if and only if $t \in T_{u n}^{P}$, the unitary part of $T^{P}$. For the classification of the dual it remains to decompose all tempered parabolically induced representations and to determine when they have constituents in common.

These phenomena are governed by intertwining operators between such representations $\mathrm{Opd} 2$, Section 4]. It is already nontrivial to show that these operators are well defined on all $\pi(\xi)$ with $t \in T_{u n}^{P}$, and it is even more difficult to see that they span $\operatorname{Hom}_{\mathcal{H}}\left(\xi, \xi^{\prime}\right)$. For reductive groups this is known as Harish-Chandra's completeness theorem, and for affine Hecke algebras it is a deep result due to Delorme and Opdam DeOp1. The intertwining operators can be collected in a groupoid $\mathcal{G}$, which acts on the space $\Xi$ of induction data $(P, \delta, t)$. With these tools one can obtain a partial classification of the dual of $\mathcal{H}(\mathcal{R}, q)$ :

Theorem 3 (see Theorem 3.3.2). There exists a natural map $\operatorname{Irr}(\mathcal{H}(\mathcal{R}, q)) \rightarrow$ $\Xi / \mathcal{G}, \rho \mapsto \mathcal{G} \xi^{+}(\rho)$, such that:

- the map is surjective and has finite fibers;

- $\rho$ is a subquotient of $\pi\left(\xi^{+}(\rho)\right)$;

- $\rho$ does not occur in $\pi(\xi)$ when $\xi$ is larger than $\xi^{+}(\rho)$, where the size of $\xi=(P, \delta, t)$ is the norm of the absolute value of the central character of $\delta$.

We note that this part of the paper is rather similar to [Sol5] for graded Hecke algebras. The results here are somewhat stronger, and most of them cannot be derived quickly from their counterparts in [Sol5].

Yet all this does not suffice for Theorem 1 because we do not have much control over the number of irreducible constituents of parabolically induced representations. Ultimately the proof of Theorem 1 is reduced to irreducible tempered representations with central character in $\operatorname{Hom}_{\mathbb{Z}}\left(X, \mathbb{R}_{>0}\right)$. The author dealt with this case in Sol6, via the periodic cyclic homology of graded Hecke algebras.

What we discussed so far corresponds more or less to Sections 1-3 of the article. From Theorem 1 to Theorem 2 is not a long journey, but we put Theorem 2 at 
the end of the paper because we prove it together with other parts of the ABPconjecture.

Sections 4 and 5 are of a more analytic nature. The main object of study is the Schwartz algebra $\mathcal{S}(\mathcal{R}, q)$ of $\mathcal{H}(\mathcal{R}, q)$ Opd2, DeOp1, the analogue of the HarishChandra-Schwartz algebra of a reductive $p$-adic group. By construction a $\mathcal{H}(\mathcal{R}, q)$ representation extends continuously to $\mathcal{S}(\mathcal{R}, q)$ if and only if it is tempered. The Schwartz algebra is the ideal tool for the harmonic analysis of affine Hecke algebras, among others because it admits a very nice Plancherel theorem (due to Delorme and Opdam, see DeOp1] or Theorem [3.2.2), because the discrete series of $\mathcal{H}(\mathcal{R}, q)$ is really discrete in the dual of $\mathcal{S}(\mathcal{R}, q)$, and because the inclusion $\mathcal{H}(\mathcal{R}, q) \rightarrow \mathcal{S}(\mathcal{R}, q)$ preserves Ext-groups of tempered representations OpSo1.

If we vary the parameter function $q$, we obtain families of algebras $\mathcal{H}(\mathcal{R}, q)$ and $\mathcal{S}(\mathcal{R}, q)$. It is natural to try to connect the representation theory of $\mathcal{H}(\mathcal{R}, q)$ with that of $\mathcal{H}\left(\mathcal{R}, q^{\prime}\right)$ for $q^{\prime}$ close to $q$. For general parameter deformations this is too difficult at present, but we can achieve it for deformations of the form $q \mapsto q^{\epsilon}$ with $\epsilon \in \mathbb{R}$. On central characters of representations, this "scaling" of $q$ fits well with the map

$$
\sigma_{\epsilon}: T \rightarrow T, t \mapsto t|t|^{\epsilon-1} .
$$

Notice that $\sigma_{\epsilon}(t)=t$ for $t \in T_{u n}=\operatorname{Hom}_{\mathbb{Z}}\left(X, S^{1}\right)$. Let $\left\{N_{w}: w \in W\right\}$ be the standard basis of $\mathcal{H}(\mathcal{R}, q)$ and let $\operatorname{Mod}_{f, W_{0} t}(\mathcal{H}(\mathcal{R}, q))$ be the category of finite dimensional $\mathcal{H}(\mathcal{R}, q)$-representations with central character $W_{0} t \in T / W_{0}$.

Theorem 4 (see Corollary 4.2.2). There exists a family of additive functors,

$$
\tilde{\sigma}_{\epsilon, t}: \operatorname{Mod}_{f, W_{0} t}(\mathcal{H}(\mathcal{R}, q)) \rightarrow \operatorname{Mod}_{f, W_{0} \sigma_{\epsilon}(t)}\left(\mathcal{H}\left(\mathcal{R}, q^{\epsilon}\right)\right) \quad \epsilon \in[-1,1],
$$

such that:

- for all $(\pi, V) \in \operatorname{Mod}_{f, W_{0} t}(\mathcal{H}(\mathcal{R}, q))$ and all $w \in W$, the map $\epsilon \mapsto \tilde{\sigma}_{\epsilon, t}(\pi)\left(N_{w}\right)$ $\in \operatorname{End}_{\mathbb{C}}(V)$ is analytic;

- for $\epsilon \neq 0, \tilde{\sigma}_{\epsilon, t}$ is an equivalence of categories;

- $\tilde{\sigma}_{\epsilon, t}$ preserves unitarity.

For $\epsilon>0$ this can already be found in $\mathrm{Opd} 2$, the most remarkable part is precisely that it extends continuously to $\epsilon=0$, that is, to the algebra $\mathcal{H}\left(\mathcal{R}, q^{0}\right)=$ $\mathbb{C}[W]$. In general the functors $\tilde{\sigma}_{\epsilon, t}$ cannot be constructed if we work only inside the algebras $\mathcal{H}\left(\mathcal{R}, q^{\epsilon}\right)$, they are obtained via localizations of these algebras at certain sets of central characters. We can do much better if we replace the algebras by their Schwartz completions:

Theorem 5 (see Theorem 4.4.2). For $\epsilon \in[0,1]$ there exist homomorphisms of Fréchet ${ }^{*}$-algebras $\zeta_{\epsilon}: \mathcal{S}\left(\mathcal{R}, q^{\epsilon}\right) \rightarrow \mathcal{S}(\mathcal{R}, q)$, such that:

- $\zeta_{\epsilon}$ is an isomorphism for all $\epsilon>0$, and $\zeta_{1}$ is the identity;

- for all $w \in W$ the map $\epsilon \mapsto \zeta_{\epsilon}\left(N_{w}\right)$ is piecewise analytic;

- for every irreducible tempered $\mathcal{H}(\mathcal{R}, q)$-representation $\pi$ with central character $W_{0} t$, the $\mathcal{S}\left(\mathcal{R}, q^{\epsilon}\right)$-representations $\tilde{\sigma}_{\epsilon, t}\left(N_{w}\right)$ and $\pi \circ \zeta_{\epsilon}$ are equivalent.

There are some similarities with the role played by Lusztig's asymptotic Hecke algebra [Lus3] in KaLu2. In both settings an algebra is constructed, which contains $\mathcal{H}(\mathcal{R}, q)$ for a family of parameter functions $q$. The asymptotic Hecke algebra is of finite type over $\mathcal{O}\left(T / W_{0}\right)$, so it is only a little larger than $\mathcal{H}(\mathcal{R}, q)$. So far it has only been constructed for equal parameter functions $q$, but Lusztig [Lus8] 
conjectures that it also exists for unequal parameter functions. On the hand, the algebra $\mathcal{S}(\mathcal{R}, q)$ is of finite type over $C^{\infty}\left(T_{u n}\right)$, so it is much larger than $\mathcal{H}(\mathcal{R}, q)$. Although $\zeta_{\epsilon}$ is an isomorphism for $\epsilon \in(0,1]$, the algebras $\mathcal{H}\left(\mathcal{R}, q^{\epsilon}\right)$ are embedded in $\mathcal{S}(\mathcal{R}, q)$ in a nontrivial way, in most cases $\zeta_{\epsilon}\left(\mathcal{H}\left(\mathcal{R}, q^{\epsilon}\right)\right)$ is not contained in $\mathcal{H}(\mathcal{R}, q)$.

Of particular interest is the homomorphism

$$
\zeta_{0}: \mathcal{S}(W)=\mathcal{S}\left(\mathcal{R}, q^{0}\right) \rightarrow \mathcal{S}(\mathcal{R}, q) .
$$

It cannot be an isomorphism, but it is injective and for all irreducible tempered $\mathcal{H}(\mathcal{R}, q)$-representations $\pi$ with central character $W_{0} t$ we have $\pi \circ \zeta_{0} \cong \tilde{\sigma}_{0, t}(\pi) \cong$ $\zeta^{*}(\pi)$. Together with Theorem 1 this results in:

Corollary 6 (see Corollary 4.4.3). The functor $\operatorname{Mod}(\mathcal{S}(\mathcal{R}, q)) \rightarrow \operatorname{Mod}(\mathcal{S}(W))$ : $\pi \mapsto \pi \circ \zeta_{0}$ induces an isomorphism between the Grothendieck groups of finite dimensional representations, tensored with $\mathbb{Q}$.

So Theorem 1 does not stand alone, but forms the end of a continuous family of representations (of a family of algebras). Actually the author first discovered the algebra homomorphism $\zeta_{0}$ and only later realized that the corresponding map on representations can also be obtained in another way, thus gaining in naturality.

Apart from representation theory, the aforementioned results have some interesting consequences in the noncommutative geometry of affine Hecke algebras. Let $C^{*}(\mathcal{R}, q)$ be the $C^{*}$-completion of $\mathcal{H}(\mathcal{R}, q)$. It contains $\mathcal{S}(\mathcal{R}, q)$ and $\zeta_{\epsilon}$ extends to a $C^{*}$-algebra homomorphism $\zeta_{\epsilon}: C^{*}\left(\mathcal{R}, q^{\epsilon}\right) \rightarrow C^{*}(\mathcal{R}, q)$, for which Theorem 5 remains valid. It follows quickly from this and Corollary 6 that $\zeta_{0}$ induces an isomorphism on topological $K$-theory; see Theorem 5.1.4. More precisely,

$$
K_{*}\left(\zeta_{0}\right) \otimes \operatorname{id}_{\mathbb{Q}}: K_{*}\left(C^{*}(W) \rtimes \Gamma\right) \otimes_{\mathbb{Z}} \mathbb{Q} \rightarrow K_{*}\left(C^{*}(\mathcal{R}, q) \rtimes \Gamma\right) \otimes_{\mathbb{Z}} \mathbb{Q}
$$

is an isomorphism, while for equal parameters the argument also goes through without $\otimes_{\mathbb{Z}} \mathbb{Q}$. This solves a conjecture that was posed first by Higson and Plymen Ply1, BCH.

Furthermore, $C^{*}(\mathcal{R}, q)$ and $\mathcal{S}(\mathcal{R}, q)$ have the same topological $K$-theory, and via the Chern character the complexification of the latter is isomorphic to the periodic cyclic homology of $\mathcal{S}(\mathcal{R}, q)$. As already proved in Sol4, $\mathcal{H}(\mathcal{R}, q)$ and $\mathcal{S}(\mathcal{R}, q)$ have the same periodic cyclic homology, so we obtain a commutative diagram

$$
\begin{aligned}
& H P_{*}(\mathbb{C}[W]) \rightarrow H P_{*}(\mathcal{S}(W)) \leftarrow K_{*}(\mathcal{S}(W)) \otimes_{\mathbb{Z}} \mathbb{C} \rightarrow K_{*}\left(C^{*}(W)\right) \otimes_{\mathbb{Z}} \mathbb{C} \\
& \downarrow \quad \downarrow H P_{*}\left(\zeta_{0}\right) \quad \downarrow K_{*}\left(\zeta_{0}\right) \quad \downarrow K_{*}\left(\zeta_{0}\right) \\
& H P_{*}(\mathcal{H}(\mathcal{R}, q)) \rightarrow H P_{*}(\mathcal{S}(\mathcal{R}, q)) \leftarrow K_{*}(\mathcal{S}(\mathcal{R}, q)) \otimes_{\mathbb{Z}} \mathbb{C} \rightarrow K_{*}\left(C^{*}(\mathcal{R}, q)\right) \otimes_{\mathbb{Z}} \mathbb{C},
\end{aligned}
$$

where all the arrows are natural isomorphisms (see Corollary 5.2.2). Notice that the Schwartz algebra $\mathcal{S}(\mathcal{R}, q)$ forms a bridge between the purely algebraic $\mathcal{H}(\mathcal{R}, q)$ and the much more analytic $C^{*}(\mathcal{R}, q)$.

For the sake of clarity, the introduction is written in less generality than the actual paper. Most notably, we can always extend our affine Hecke algebras by a group $\Gamma$ of automorphisms of the Dynkin diagram of $\mathcal{R}$. On the one hand this generality is forced upon us, in particular, by Lusztig's first reduction theorem (see Theorem 2.1.2), which necessarily involves diagram automorphisms. On the other hand, one advantage of having $\mathcal{H}(\mathcal{R}, q) \rtimes \Gamma$ instead of just $\mathcal{H}(\mathcal{R}, q)$ is that our proof of the Aubert-Baum-Plymen conjecture applies to clearly more Bernstein components of reductive $p$-adic groups.

For most of the results of this paper, the extension from $\mathcal{H}(\mathcal{R}, q)$ to $\mathcal{H}(\mathcal{R}, q) \rtimes \Gamma$ is easy, mainly a matter of some extra notation. An exception is the Langlands 
classification, which hitherto was only known for commutative groups of diagram automorphisms [BaJa1]. In our generalization (see Corollary 2.2.5) we add a new ingredient to the Langlands data, and we show how to save the uniqueness part.

A substantial part of this article is based on the author's PhD thesis [Sol3], which was written under the supervision of Opdam. We refrain from indicating all the things that stem from [Sol3] among others because some of proofs in Sol3] were not worked out with the accuracy needed for research papers. Moreover, in the years after writing this thesis many additional insights were obtained, so that in the end actually no part of [Sol3] reached this article unscathed. The technical Section 4 comes closest. It should also be mentioned that the conjecture (2) formed a central part of the author's $\mathrm{PhD}$ research. At that time it was still too difficult for the author, mainly because Theorem 1 was not yet available.

\section{Preliminaries}

This section serves mainly to introduce some definitions and notation that we will use later on. The results that we recall can be found in several other sources, such as Lus6, Ree1, Opd2. By default, our affine Hecke algebras are endowed with unequal parameters and may be extended with a group of automorphisms of the underlying root datum.

In the section dedicated to $p$-adic groups we recall what is known about the (conjectural) relation between Bernstein components and affine Hecke algebras. On one hand, this motivates the generality that we work in, on the other hand we will use it in Section [5.4 to translate a conjecture of Aubert, Baum and Plymen to the setting of affine Hecke algebras.

1.1. Root systems. Let $\mathfrak{a}$ be a finite dimensional real vector space and let $\mathfrak{a}^{*}$ be its dual. Let $Y \subset \mathfrak{a}$ be a lattice and $X=\operatorname{Hom}_{\mathbb{Z}}(Y, \mathbb{Z}) \subset \mathfrak{a}^{*}$ the dual lattice. Let

$$
\mathcal{R}=\left(X, R_{0}, Y, R_{0}^{\vee}, F_{0}\right)
$$

be a based root datum. Thus $R_{0}$ is a reduced root system in $X, R_{0}^{\vee} \subset Y$ is the dual root system, $F_{0}$ is a basis of $R_{0}$ and the set of positive roots is denoted $R_{0}^{+}$. Furthermore, we are given a bijection $R_{0} \rightarrow R_{0}^{\vee}, \alpha \mapsto \alpha^{\vee}$ such that $\left\langle\alpha, \alpha^{\vee}\right\rangle=2$ and such that the corresponding reflections $s_{\alpha}: X \rightarrow X$ (resp. $s_{\alpha}^{\vee}: Y \rightarrow Y$ ) stabilize $R_{0}$ (resp. $R_{0}^{\vee}$ ). We do not assume that $R_{0}$ spans $\mathfrak{a}^{*}$.

The reflections $s_{\alpha}$ generate the Weyl group $W_{0}=W\left(R_{0}\right)$ of $R_{0}$, and $S_{0}:=\left\{s_{\alpha}\right.$ : $\left.\alpha \in F_{0}\right\}$ is the collection of simple reflections. We have the affine Weyl group and its extended version:

$$
\begin{aligned}
& W^{\text {aff }}=W^{\text {aff }}(\mathcal{R})=\mathbb{Z} R_{0} \rtimes W_{0}, \\
& W=W(\mathcal{R})=X \rtimes W_{0} .
\end{aligned}
$$

Both can be considered as groups of affine transformations of $\mathfrak{a}^{*}$. We denote the translation corresponding to $x \in X$ by $t_{x}$. As is well known, $W^{\text {aff }}$ is a Coxeter group, and the basis of $R_{0}$ gives rise to a set $S^{\text {aff }}$ of simple (affine) reflections. More explicitly, let $F_{M}^{\vee}$ be the set of maximal elements of $R_{0}^{\vee}$, with respect to the dominance ordering coming from $F_{0}$. Then

$$
S^{\text {aff }}=S_{0} \cup\left\{t_{\alpha} s_{\alpha}: \alpha \in F_{M}\right\} .
$$


We write

$$
\begin{aligned}
& X^{+}:=\left\{x \in X:\left\langle x, \alpha^{\vee}\right\rangle \geq 0 \forall \alpha \in F_{0}\right\}, \\
& X^{-}:=\left\{x \in X:\left\langle x, \alpha^{\vee}\right\rangle \leq 0 \forall \alpha \in F_{0}\right\}=-X^{+} .
\end{aligned}
$$

It is easily seen that the center of $W$ is the lattice

$$
Z(W)=X^{+} \cap X^{-} \text {. }
$$

We say that $\mathcal{R}$ is semisimple if $Z(W)=0$ or equivalently if $R_{0}$ spans $\mathfrak{a}^{*}$. Thus a root datum is semisimple if and only if the corresponding reductive algebraic group is so.

The length function $\ell$ of the Coxeter system $\left(W^{\text {aff }}, S^{\text {aff }}\right)$ extends naturally to $W$, such that Opd1, (1.3)]

$$
\ell\left(w t_{x}\right)=\ell(w)+\sum_{\alpha \in R_{0}^{+}}\left\langle x, \alpha^{\vee}\right\rangle \quad w \in W_{0}, x \in X^{+} .
$$

The elements of length zero form a subgroup $\Omega \subset W$, and $W=W^{\text {aff }} \rtimes \Omega$. With $\mathcal{R}$ we also associate some other root systems. There is the nonreduced root system

$$
R_{n r}:=R_{0} \cup\left\{2 \alpha: \alpha^{\vee} \in 2 Y\right\} .
$$

Obviously, we put $(2 \alpha)^{\vee}=\alpha^{\vee} / 2$. Let $R_{1}$ be the reduced root system of long roots in $R_{n r}$ :

$$
R_{1}:=\left\{\alpha \in R_{n r}: \alpha^{\vee} \notin 2 Y\right\} .
$$

We denote the collection of positive roots in $R_{0}$ by $R_{0}^{+}$, and similarly for other root systems.

1.2. Affine Hecke algebras. There are three equivalent ways to introduce a complex parameter function for $\mathcal{R}$.

(1) A map $q: S^{\text {aff }} \rightarrow \mathbb{C}^{\times}$such that $q(s)=q\left(s^{\prime}\right)$ if $s$ and $s^{\prime}$ are conjugate in $W$.

(2) A function $q: W \rightarrow \mathbb{C}^{\times}$such that

$$
\begin{aligned}
& q(\omega)=1 \quad \text { if } \ell(\omega)=0, \\
& q(w v)=q(w) q(v) \quad \text { if } w, v \in W \quad \text { and } \quad \ell(w v)=\ell(w)+\ell(v) .
\end{aligned}
$$

(3) A $W_{0}$-invariant map $q: R_{n r}^{\vee} \rightarrow \mathbb{C}^{\times}, \alpha^{\vee} \mapsto q_{\alpha^{\vee}}$.

One goes from (2) to (1) by restriction, while the relation between (2) and (3) is given by

$$
\begin{array}{lll}
q_{\alpha^{\vee}}=q\left(s_{\alpha}\right)=q\left(t_{\alpha} s_{\alpha}\right) & \text { if } & \alpha \in R_{0} \cap R_{1}, \\
q_{\alpha^{\vee}}=q\left(t_{\alpha} s_{\alpha}\right) & \text { if } & \alpha \in R_{0} \backslash R_{1}, \\
q_{\alpha^{\vee} / 2}=q\left(s_{\alpha}\right) q\left(t_{\alpha} s_{\alpha}\right)^{-1} & \text { if } & \alpha \in R_{0} \backslash R_{1} .
\end{array}
$$

We speak of equal parameters if $q(s)=q\left(s^{\prime}\right) \forall s, s^{\prime} \in S^{\text {aff }}$ and of positive parameters if $q(s) \in \mathbb{R}_{>0} \forall s \in S^{\text {aff }}$.

We fix a square root $q^{1 / 2}: S^{\text {aff }} \rightarrow \mathbb{C}^{\times}$. The affine Hecke algebra $\mathcal{H}=\mathcal{H}(\mathcal{R}, q)$ is the unique associative complex algebra with basis $\left\{N_{w}: w \in W\right\}$ and multiplication rules

$$
\begin{array}{ll}
N_{w} N_{v}=N_{w v} & \text { if } \quad \ell(w v)=\ell(w)+\ell(v), \\
\left(N_{s}-q(s)^{1 / 2}\right)\left(N_{s}+q(s)^{-1 / 2}\right)=0 & \text { if } \quad s \in S^{\text {aff }} .
\end{array}
$$

In the literature one also finds this algebra defined in terms of the elements $q(s)^{1 / 2} N_{s}$, in which case the multiplication can be described without square roots. This explains why $q^{1 / 2}$ does not appear in the notation $\mathcal{H}(\mathcal{R}, q)$. 
Notice that $N_{w} \mapsto N_{w^{-1}}$ extends to a $\mathbb{C}$-linear anti-automorphism of $\mathcal{H}$, so $\mathcal{H}$ is isomorphic to its opposite algebra. The span of the $N_{w}$ with $w \in W_{0}$ is a finite dimensional Iwahori-Hecke algebra, which we denote by $\mathcal{H}\left(W_{0}, q\right)$.

Now we describe the Bernstein presentation of $\mathcal{H}$. For $x \in X^{+}$we put $\theta_{x}:=$ $N_{t_{x}}$. The corresponding semigroup morphism $X^{+} \rightarrow \mathcal{H}(\mathcal{R}, q)^{\times}$extends to a group homomorphism

$$
X \rightarrow \mathcal{H}(\mathcal{R}, q)^{\times}: x \mapsto \theta_{x} .
$$

Theorem 1.2.1 (Bernstein presentation). (a) The sets $\left\{N_{w} \theta_{x}: w \in W_{0}, x \in\right.$ $X\}$ and $\left\{\theta_{x} N_{w}: w \in W_{0}, x \in X\right\}$ are bases of $\mathcal{H}$.

(b) The subalgebra $\mathcal{A}:=\operatorname{span}\left\{\theta_{x}: x \in X\right\}$ is isomorphic to $\mathbb{C}[X]$.

(c) The center of $Z(\mathcal{H}(\mathcal{R}, q))$ of $\mathcal{H}(\mathcal{R}, q)$ is $\mathcal{A}^{W_{0}}$, where we define the action of $W_{0}$ on $\mathcal{A}$ by $w\left(\theta_{x}\right)=\theta_{w x}$.

(d) For $f \in \mathcal{A}$ and $\alpha \in F_{0} \cap R_{1}$,

$$
f N_{s_{\alpha}}-N_{s_{\alpha}} s_{\alpha}(f)=\left(q\left(s_{\alpha}\right)^{1 / 2}-q\left(s_{\alpha}\right)^{-1 / 2}\right)\left(f-s_{\alpha}(f)\right)\left(\theta_{0}-\theta_{-\alpha}\right)^{-1},
$$

while for $\alpha \in F_{0} \backslash R_{1}$ :

$$
f N_{s_{\alpha}}-N_{s_{\alpha}} s_{\alpha}(f)=\left(q\left(s_{\alpha}\right)^{1 / 2}-q\left(s_{\alpha}\right)^{-1 / 2}+\left(q_{\alpha^{\vee}}^{1 / 2}-q_{\alpha^{\vee}}^{-1 / 2}\right) \theta_{-\alpha}\right) \frac{f-s_{\alpha}(f)}{\theta_{0}-\theta_{-2 \alpha}} .
$$

Proof. These results are due to Bernstein; see [Lus6, §3].

The following lemma was claimed in the proof of [Opd1, Lemma 3.1].

Lemma 1.2.2. For $x \in X^{+}$,

$$
\operatorname{span}\left\{N_{u} \theta_{x} N_{v}: u, v \in W_{0}\right\}=\operatorname{span}\left\{N_{w}: w \in W_{0} t_{x} W_{0}\right\} .
$$

Let $W_{x}$ be the stabilizer of $x$ in $W_{0}$ and let $W^{x}$ be a set of representatives for $W_{0} / W_{x}$. Then the elements $N_{u} \theta_{x} N_{v}$ with $u \in W^{x}$ and $v \in W_{0}$ form a basis of (1.5).

Proof. By (1.1) $\ell\left(u t_{x}\right)=\ell(u)+\ell\left(t_{x}\right)$, so $N_{u} \theta_{x}=N_{u t_{x}}$. Recall the Bruhat ordering on a Coxeter group, for example from [Hum, Sections 5.9 and 5.10]. With induction to $\ell(v)$ it follows from the multiplication rules (1.4) that

$$
N_{w} N_{v}-N_{w v} \in \operatorname{span}\left\{N_{w \tilde{v}}: \tilde{v}<v \text { in the Bruhat ordering }\right\} .
$$

Hence the sets $\left\{N_{u} \theta_{x} N_{v}: v \in W_{0}\right\}$ and $\left\{N_{w}: w \in u t_{x} W_{0}\right\}$ have the same span. They have the same cardinality and by definition the latter set is linearly independent, so the former is linearly independent as well. Clearly, $W_{0} t_{x} W_{0}=$ $\bigsqcup_{u \in W^{x}} u t_{x} W_{0}$, so

$$
\begin{aligned}
& \operatorname{span}\left\{N_{w}: w \in W_{0} t_{x} W_{0}\right\}=\bigoplus_{u \in W^{x}} \operatorname{span}\left\{N_{w}: w \in u t_{x} W_{0}\right\} \\
& \quad=\bigoplus_{u \in W^{x}} \operatorname{span}\left\{N_{u} \theta_{x} N_{v}: v \in W_{0}\right\}=\operatorname{span}\left\{N_{u} \theta_{x} N_{v}: u \in W^{x}, v \in W_{0}\right\} .
\end{aligned}
$$

The number of generators on the second line side equals the dimension of the first line, so they form a basis.

Let $T$ be the complex algebraic torus

$$
T=\operatorname{Hom}_{\mathbb{Z}}\left(X, \mathbb{C}^{\times}\right) \cong Y \otimes_{\mathbb{Z}} \mathbb{C}^{\times},
$$


so $\mathcal{A} \cong \mathcal{O}(T)$ and $Z(\mathcal{H})=\mathcal{A}^{W_{0}} \cong \mathcal{O}\left(T / W_{0}\right)$. From Theorem 1.2.1 we see that $\mathcal{H}$ is of finite rank over its center. Let $\mathfrak{t}=\operatorname{Lie}(T)$ and $\mathfrak{t}^{*}$ be the complexifications of $\mathfrak{a}$ and $\mathfrak{a}^{*}$. The direct sum $\mathfrak{t}=\mathfrak{a} \oplus i \mathfrak{a}$ corresponds to the polar decomposition

$$
T=T_{r s} \times T_{u n}=\operatorname{Hom}_{\mathbb{Z}}\left(X, \mathbb{R}_{>0}\right) \times \operatorname{Hom}_{\mathbb{Z}}\left(X, S^{1}\right),
$$

where $T_{r s}$ is the real split (or positive) part of $T$ and $T_{u n}$ the unitary part. The exponential map exp $: \mathfrak{t} \rightarrow T$ is bijective on the real parts, and we denote its inverse by $\log : T_{r s} \rightarrow \mathfrak{a}$.

An important role in the harmonic analysis of $\mathcal{H}(\mathcal{R}, q)$ is played by the Macdonald $c$-functions $c_{\alpha} \in \mathbb{C}(T)$ (cf. [Lus6, 3.8] and Opd1, Section 1.7]), defined as

$$
c_{\alpha}=\frac{\theta_{\alpha}+q\left(s_{\alpha}\right)^{-1 / 2} q_{\alpha^{\vee}}^{1 / 2}}{\theta_{\alpha}+1} \frac{\theta_{\alpha}-q\left(s_{\alpha}\right)^{-1 / 2} q_{\alpha^{\vee}}^{-1 / 2}}{\theta_{\alpha}-1} .
$$

Notice that $c_{\alpha}=1$ if and only if $q\left(s_{\alpha}\right)=q\left(t_{\alpha} s_{\alpha}\right)=1$. For $\alpha \in R_{0} \cap R_{1}$ we have $q\left(s_{\alpha}\right)=q_{\alpha}$, so (1.6) simplifies to $c_{\alpha}=\left(\theta_{\alpha}-q\left(s_{\alpha}\right)^{-1}\right)\left(\theta_{\alpha}-1\right)^{-1}$. With these $c$-functions we can rephrase Theorem 1.2.1 d as

$$
f N_{s_{\alpha}}-N_{s_{\alpha}} s_{\alpha}(f)=q\left(s_{\alpha}\right)^{-1 / 2}\left(f-s_{\alpha}(f)\right)\left(q\left(s_{\alpha}\right) c_{\alpha}-1\right) .
$$

An automorphism of the Dynkin diagram of the based root system $\left(R_{0}, F_{0}\right)$ is a bijection $\gamma: F_{0} \rightarrow F_{0}$ such that

$$
\left\langle\gamma(\alpha), \gamma(\beta)^{\vee}\right\rangle=\left\langle\alpha, \beta^{\vee}\right\rangle \quad \forall \alpha, \beta \in F_{0} .
$$

Such a $\gamma$ naturally induces automorphisms of $R_{0}, R_{0}^{\vee}, W_{0}$ and $W^{\text {aff }}$. It is easy to classify all diagram automorphisms of $\left(R_{0}, F_{0}\right)$ : they permute the irreducible components of $R_{0}$ of a given type, and the diagram automorphisms of a connected Dynkin diagram can be seen immediately.

We will assume that the action of $\gamma$ on $W^{\text {aff }}$ has been extended in some way to $W$. For example, this is the case if $\gamma$ belongs to the Weyl group of some larger root system contained in $X$. We regard two diagram automorphisms as the same if and only if their actions on $W$ are equal.

Let $\Gamma$ be a finite group of diagram automorphisms of $\left(R_{0}, F_{0}\right)$ and assume that $q_{\alpha^{\vee}}=q_{\gamma\left(\alpha^{\vee}\right)}$ for all $\gamma \in \Gamma, \alpha \in R_{n r}$. Then $\Gamma$ acts on $\mathcal{H}$ by algebra automorphisms $\psi_{\gamma}$ that satisfy

$$
\begin{aligned}
& \psi_{\gamma}\left(N_{w}\right)=N_{\gamma(w)} \quad w \in W, \\
& \psi_{\gamma}\left(\theta_{x}\right)=\theta_{\gamma(x)} \quad x \in X .
\end{aligned}
$$

Hence one can form the crossed product algebra $\Gamma \ltimes \mathcal{H}=\mathcal{H} \rtimes \Gamma$, whose natural basis is indexed by the group $\left(X \rtimes W_{0}\right) \rtimes \Gamma=X \rtimes\left(W_{0} \rtimes \Gamma\right)$. It follows easily from (1.8) and Theorem 1.2.1.c that $Z(\mathcal{H} \rtimes \Gamma)=\mathcal{A}^{W_{0} \rtimes \Gamma}$. We say that the central character of an (irreducible) $\mathcal{H} \rtimes \Gamma$-representation is positive if it lies in $T^{r s} /\left(W_{0} \rtimes \Gamma\right)$.

We always assume that we have an $\Gamma \ltimes W_{0}$-invariant inner product on $\mathfrak{a}$. The length function of $W^{\text {aff }}$ also extends to $X \rtimes W_{0} \rtimes \Gamma$, and the subgroup of elements of length zero becomes

$$
\{w \in W \rtimes \Gamma: \ell(w)=0\}=\Gamma \ltimes\{w \in W: \ell(w)=0\}=\Gamma \ltimes \Omega .
$$

More generally, one can consider a finite group $\Gamma^{\prime}$ that acts on $\mathcal{R}$ by diagram automorphisms. Then the center of $\mathcal{H} \rtimes \Gamma^{\prime}$ can be larger than $\mathcal{A}^{W_{0} \rtimes \Gamma^{\prime}}$, but apart from that, the structure is the same. 
Another variation arises when $\Gamma \rightarrow \operatorname{Aut}(\mathcal{H})$ is not a group homomorphism, but a homomorphism twisted by a 2-cocycle $\kappa: \Gamma \times \Gamma \rightarrow \mathbb{C}^{\times}$. Instead of $\mathcal{H} \rtimes \Gamma$ one can construct the algebra $\mathcal{H} \otimes \mathbb{C}[\Gamma, \kappa]$, whose multiplication is defined by

$$
\begin{array}{ll}
N_{\gamma} N_{\gamma^{\prime}} & =\kappa\left(\gamma, \gamma^{\prime}\right) N_{\gamma \gamma^{\prime}}, \quad \gamma, \gamma^{\prime} \in \Gamma, h \in \mathcal{H} . \\
N_{\gamma} h N_{\gamma}^{-1} & =\gamma(h),
\end{array}
$$

By [Mor, Section 7] such algebras can appear in relevant examples, although no explicit nontrivial cocycles are known. Let $\Gamma^{*}$ be the Schur multiplier of $\Gamma$, also known as representation group CuRe. It is a central extension of $\Gamma$ that classifies projective $\Gamma$-representations, and its group algebra $\mathbb{C}\left[\Gamma^{*}\right]$ contains $\mathbb{C}[\Gamma, \kappa]$ as a direct summand. The algebra $\mathcal{H} \rtimes \Gamma^{*}$ is well defined and contains $\mathcal{H} \otimes \mathbb{C}[\Gamma, \kappa]$ as a direct summand. Thus we can reduce the study of affine Hecke algebras with twisted group actions to the case of honest group actions.

1.3. Graded Hecke algebras. Graded Hecke algebras are also known as degenerate (affine) Hecke algebras. They were introduced by Lusztig in [Lus6]. We call

$$
\tilde{\mathcal{R}}=\left(\mathfrak{a}^{*}, R_{0}, \mathfrak{a}, R_{0}^{\vee}, F_{0}\right)
$$

a degenerate root datum. We pick complex numbers $k_{\alpha}$ for $\alpha \in F_{0}$, such that $k_{\alpha}=k_{\beta}$ if $\alpha$ and $\beta$ are in the same $W_{0}$-orbit. The graded Hecke algebra associated to these data is the complex vector space

$$
\mathbb{H}=\mathbb{H}(\tilde{\mathcal{R}}, k)=S\left(\mathfrak{t}^{*}\right) \otimes \mathbb{C}\left[W_{0}\right],
$$

with multiplication defined by the following rules:

- $\mathbb{C}\left[W_{0}\right]$ and $S\left(\mathfrak{t}^{*}\right)$ are canonically embedded as subalgebras;

- for $x \in \mathfrak{t}^{*}$ and $s_{\alpha} \in S$ we have the cross relation

$$
x \cdot s_{\alpha}-s_{\alpha} \cdot s_{\alpha}(x)=k_{\alpha}\left\langle x, \alpha^{\vee}\right\rangle .
$$

Multiplication with any $\epsilon \in \mathbb{C}^{\times}$defines a bijection $m_{\epsilon}: \mathfrak{t}^{*} \rightarrow \mathfrak{t}^{*}$, which clearly extends to an algebra automorphism of $S\left(\mathfrak{t}^{*}\right)$. From the cross relation (1.10) we see that it extends even further to an algebra isomorphism,

$$
m_{\epsilon}: \mathbb{H}(\tilde{\mathcal{R}}, \epsilon k) \rightarrow \mathbb{H}(\tilde{\mathcal{R}}, k),
$$

which is the identity on $\mathbb{C}\left[W_{0}\right]$.

The $c$-functions are considerably easier than for affine Hecke algebras:

$$
\tilde{c}_{\alpha}=\left(\alpha+k_{\alpha}\right) \alpha^{-1}=1+k_{\alpha} \alpha^{-1} \in \mathbb{C}(\mathfrak{t}) .
$$

They can be used to rewrite (1.10) as

$$
x s_{\alpha}-s_{\alpha} s_{\alpha}(x)=\left(x-s_{\alpha}(x)\right)\left(\tilde{c}_{\alpha}-1\right) \quad \alpha \in F_{0}, x \in \mathfrak{t}^{*} .
$$

Let $\Gamma$ be a group of diagram automorphisms of $\tilde{\mathcal{R}}$ and assume that $k_{\gamma(\alpha)}=k_{\alpha}$ for all $\alpha \in R_{0}, \gamma \in \Gamma$. Then $\Gamma$ acts on $\mathbb{H}$ by the algebra automorphisms

$$
\begin{aligned}
& \psi_{\gamma}: \mathbb{H} \rightarrow \mathbb{H}, \\
& \psi_{\gamma}\left(x s_{\alpha}\right)=\gamma(x) s_{\gamma(\alpha)} \quad x \in \mathfrak{t}^{*}, \alpha \in \Pi .
\end{aligned}
$$

By [Sol6, Proposition 5.1.a] the center of the resulting crossed product algebra is

$$
Z(\mathbb{H} \rtimes \Gamma)=S\left(\mathfrak{t}^{*}\right)^{W_{0} \rtimes \Gamma}=\mathcal{O}\left(\mathfrak{t} /\left(W_{0} \rtimes \Gamma\right)\right) .
$$

We say that the central character of an $\mathbb{H} \rtimes \Gamma$-representation is real if it lies in $\mathfrak{a} /\left(W_{0} \rtimes \Gamma\right)$. 
1.4. Parabolic subalgebras. For a set of simple roots $P \subset F_{0}$ we introduce the notations

$$
\begin{array}{ll}
R_{P}=\mathbb{Q} P \cap R_{0} & R_{P}^{\vee}=\mathbb{Q} R_{P}^{\vee} \cap R_{0}^{\vee}, \\
\mathfrak{a}_{P}=\mathbb{R} P^{\vee} & \mathfrak{a}^{P}=\left(\mathfrak{a}_{P}^{*}\right)^{\perp}, \\
\mathfrak{a}_{P}^{*}=\mathbb{R} P & \mathfrak{a}^{P *}=\left(\mathfrak{a}_{P}\right)^{\perp}, \\
\mathfrak{t}_{P}=\mathbb{C} P^{\vee} & \mathfrak{t}^{P}=\left(\mathfrak{t}_{P}^{*}\right)^{\perp}, \\
\mathfrak{t}_{P}^{*}=\mathbb{C} P & \mathfrak{t}^{P *}=\left(\mathfrak{t}_{P}\right)^{\perp}, \\
X_{P}=X /\left(X \cap\left(P^{\vee}\right)^{\perp}\right) & X^{P}=X /(X \cap \mathbb{Q} P), \\
Y_{P}=Y \cap \mathbb{Q} P^{\vee} & Y^{P}=Y \cap P^{\perp}, \\
T_{P}=\operatorname{Hom}_{\mathbb{Z}}\left(X_{P}, \mathbb{C}^{\times}\right) & T^{P}=\operatorname{Hom}_{\mathbb{Z}}\left(X^{P}, \mathbb{C}^{\times}\right), \\
\mathcal{R}_{P}=\left(X_{P}, R_{P}, Y_{P}, R_{P}^{\vee}, P\right) & \mathcal{R}^{P}=\left(X, R_{P}, Y, R_{P}^{\vee}, P\right), \\
\tilde{\mathcal{R}}_{P}=\left(\mathfrak{a}_{P}^{*}, R_{P}, \mathfrak{a}_{P}, R_{P}^{\vee}, P\right) & \tilde{\mathcal{R}}^{P}=\left(\mathfrak{a}^{*}, R_{P}, \mathfrak{a}, R_{P}^{\vee}, P\right) .
\end{array}
$$

We denote the image of $x \in X$ in $X_{P}$ by $x_{P}$. Although $T_{r s}=T_{P, r s} \times T_{r s}^{P}$, the product $T_{u n}=T_{P, u n} T_{u n}^{P}$ is not direct, because the intersection

$$
K_{P}:=T_{P, u n} \cap T_{u n}^{P}=T_{P} \cap T^{P}
$$

can have more than one element (but only finitely many).

We define parameter functions $q_{P}$ and $q^{P}$ on the root data $\mathcal{R}_{P}$ and $\mathcal{R}^{P}$, as follows. Restrict $q$ to a function on $\left(R_{P}\right)_{n r}^{\vee}$ and use (1.3) to extend it to $W\left(\mathcal{R}_{P}\right)$ and $W\left(\mathcal{R}^{P}\right)$. Similarly the restriction of $k$ to $P$ is a parameter function for the degenerate root data $\tilde{\mathcal{R}}_{P}$ and $\tilde{\mathcal{R}}^{P}$, and we denote it by $k_{P}$ or $k^{P}$. Now we can define the parabolic subalgebras

$$
\begin{array}{ll}
\mathcal{H}_{P}=\mathcal{H}\left(\mathcal{R}_{P}, q_{P}\right), & \mathcal{H}^{P}=\mathcal{H}\left(\mathcal{R}^{P}, q^{P}\right), \\
\mathbb{H}_{P}=\mathbb{H}\left(\tilde{\mathcal{R}}_{P}, k_{P}\right), & \mathbb{H}^{P}=\mathbb{H}\left(\tilde{\mathcal{R}}^{P}, k^{P}\right) .
\end{array}
$$

We notice that $\mathbb{H}^{P}=S\left(\mathfrak{t}^{P *}\right) \otimes \mathbb{H}_{P}$, a tensor product of algebras. Despite our terminology $\mathcal{H}^{P}$ and $\mathcal{H}_{P}$ are not subalgebras of $\mathcal{H}$, but they are close. Namely, $\mathcal{H}\left(\mathcal{R}^{P}, q^{P}\right)$ is isomorphic to the subalgebra of $\mathcal{H}(\mathcal{R}, q)$ generated by $\mathcal{A}$ and $\mathcal{H}\left(W\left(R_{P}\right), q_{P}\right)$. We denote the image of $x \in X$ in $X_{P}$ by $x_{P}$ and we let $\mathcal{A}_{P} \subset \mathcal{H}_{P}$ be the commutative subalgebra spanned by $\left\{\theta_{x_{P}}: x_{P} \in X_{P}\right\}$. There is natural surjective quotient map

$$
\mathcal{H}^{P} \rightarrow \mathcal{H}_{P}: \theta_{x} N_{w} \mapsto \theta_{x_{P}} N_{w} .
$$

Suppose that $\gamma \in \Gamma \ltimes W_{0}$ satisfies $\gamma(P)=Q \subseteq F_{0}$. Then there are algebra isomorphisms

$$
\begin{array}{lll}
\psi_{\gamma}: \mathcal{H}_{P} \rightarrow \mathcal{H}_{Q}, & \theta_{x_{P}} N_{w} & \mapsto \theta_{\gamma\left(x_{P}\right)} N_{\gamma w \gamma^{-1}}, \\
\psi_{\gamma}: \mathcal{H}^{P} \rightarrow \mathcal{H}^{Q}, & \theta_{x} N_{w} & \mapsto \theta_{\gamma x} N_{\gamma w \gamma^{-1}}, \\
\psi_{\gamma}: \mathbb{H}_{P} \rightarrow \mathbb{H}_{Q}, \quad f_{P} w & \mapsto\left(f_{P} \circ \gamma^{-1}\right) w, \\
\psi_{\gamma}: \mathbb{H}_{P} \rightarrow \mathbb{H}_{Q}, \quad f w & \mapsto\left(f \circ \gamma^{-1}\right) w,
\end{array}
$$

where $f_{P} \in \mathcal{O}\left(\mathfrak{t}_{P}\right)$ and $f \in \mathcal{O}(\mathfrak{t})$. Sometimes we will abbreviate $W \rtimes \Gamma$ to $W^{\prime}$ and $W_{0} \rtimes \Gamma$ to $W_{0}^{\prime}$. For example, the group

$$
W_{P}^{\prime}:=\left\{\gamma \in \Gamma \ltimes W_{0}: \gamma(P)=P\right\}
$$

acts on the algebras $\mathcal{H}_{P}$ and $\mathcal{H}^{P}$. Although $W_{F_{0}}^{\prime}=\Gamma$, for proper subsets $P \subsetneq F_{0}$ the group $W_{P}^{\prime}$ need not be contained in $\Gamma$. In other words, in general $W_{P}^{\prime}$ strictly contains the group

$$
\Gamma_{P}:=\{\gamma \in \Gamma: \gamma(P)=P\}=W_{P}^{\prime} \cap \Gamma .
$$


To avoid confusion we do not use the notation $W_{P}$. Instead the parabolic subgroup of $W_{0}$ generated by $\left\{s_{\alpha}: \alpha \in P\right\}$ will be denoted $W\left(R_{P}\right)$. Suppose that $\gamma \in W_{0}^{\prime}$ stabilizes either the root system $R_{P}$, the lattice $\mathbb{Z} P$ or the vector space $\mathbb{Q} P \subset \mathfrak{a}^{*}$. Then $\gamma(P)$ is a basis of $R_{P}$, so $\gamma(P)=w(P)$ and $w^{-1} \gamma \in W_{P}^{\prime}$ for a unique $w \in$ $W\left(R_{P}\right)$. Therefore

$$
W_{\mathbb{Z} P}^{\prime}:=\left\{\gamma \in W_{0}^{\prime}: \gamma(\mathbb{Z} P)=\mathbb{Z} P\right\} \text { equals } W\left(R_{P}\right) \rtimes W_{P}^{\prime} .
$$

For all $x \in X$ and $\alpha \in P$ we have

$$
x-s_{\alpha}(x)=\left\langle x, \alpha^{\vee}\right\rangle \alpha \in \mathbb{Z} P,
$$

so $t\left(s_{\alpha}(x)\right)=t(x)$ for all $t \in T^{P}$. Hence $t(w(x))=t(x)$ for all $w \in W\left(R_{P}\right)$, and we can define an algebra automorphism

$$
\phi_{t}: \mathcal{H}^{P} \rightarrow \mathcal{H}^{P}, \quad \phi_{t}\left(\theta_{x} N_{w}\right)=t(x) \theta_{x} N_{w} \quad t \in T^{P} .
$$

In particular, for $t \in K_{P}$ this descends to an algebra automorphism

$$
\psi_{t}: \mathcal{H}_{P} \rightarrow \mathcal{H}_{P}, \quad \theta_{x_{P}} N_{w} \mapsto t\left(x_{P}\right) \theta_{x_{P}} N_{w} \quad t \in K_{P} .
$$

We can regard any representation $\left(\sigma, V_{\sigma}\right)$ of $\mathcal{H}\left(\mathcal{R}_{P}, q_{P}\right)$ as a representation of $\mathcal{H}\left(\mathcal{R}^{P}, q^{P}\right)$ via the quotient map (1.16). Thus we can construct the $\mathcal{H}$-representation

$$
\pi(P, \sigma, t):=\operatorname{Ind}_{\mathcal{H}\left(\mathcal{R}^{P}, q^{P}\right)}^{\mathcal{H}(\mathcal{R}, q)}\left(\sigma \circ \phi_{t}\right) .
$$

Representations of this form are said to be parabolically induced. Similarly, for any $\mathbb{H}_{P}$-representation $\left(\rho, V_{\rho}\right)$ and any $\lambda \in \mathfrak{t}^{P}$ there is an $\mathbb{H}^{P}$-representation $\left(\rho_{\lambda}, V_{\rho} \otimes\right.$ $\left.\mathbb{C}_{\lambda}\right)$. The corresponding parabolically induced representation is

$$
\pi(P, \rho, \lambda):=\operatorname{Ind}_{\mathbb{H}^{P}}^{\mathbb{H}}\left(\rho_{\lambda}\right)=\operatorname{Ind}_{\mathbb{H}^{P}}^{\mathbb{H}}\left(V_{\rho} \otimes \mathbb{C}_{\lambda}\right) .
$$

1.5. Analytic localization. A common technique in the study of Hecke algebras is localization at one or more characters of the center. There are several ways to implement this. Lusztig Lus6 takes a maximal ideal $I$ of $Z(\mathcal{H})$ and completes $\mathcal{H}$ with respect to the powers of this ideal. This has the effect of considering only those $\mathcal{H}$-representations which admit the central character corresponding to $I$.

For reasons that will become clear only in Section 4 , we prefer to localize with analytic functions on subvarieties of $T / W_{0}^{\prime}$. Let $U \subset T$ be a nonempty $W_{0}^{\prime}$-invariant subset and let $C^{a n}(U)$ (respectively $C^{m e}(U)$ ) be the algebra of holomorphic (respectively meromorphic) functions on $U$. There is a natural embedding

$$
Z(\mathcal{H} \rtimes \Gamma)=\mathcal{A}^{W_{0}^{\prime}} \cong \mathcal{O}(T)^{W_{0}^{\prime}} \rightarrow C^{a n}(U)^{W_{0}^{\prime}}
$$

and isomorphisms of topological algebras

$$
C^{a n}(U)^{W_{0}^{\prime}} \otimes_{\mathcal{A}^{W_{0}^{\prime}}} \mathcal{A} \cong C^{a n}(U), \quad C^{m e}(U)^{W_{0}^{\prime}} \otimes_{\mathcal{A}^{W_{0}^{\prime}}} \mathcal{A} \cong C^{m e}(U) .
$$

Thus we can construct the algebras

$$
\begin{aligned}
& \mathcal{H}^{a n}(U) \rtimes \Gamma:=C^{a n}(U)^{W_{0}^{\prime}} \otimes_{Z(\mathcal{H} \rtimes \Gamma)} \mathcal{H} \rtimes \Gamma \cong C^{a n}(U) \otimes_{\mathbb{C}} \mathbb{C}\left[W_{0}^{\prime}\right], \\
& \mathcal{H}^{m e}(U) \rtimes \Gamma:=C^{m e}(U)^{W_{0}^{\prime}} \otimes_{Z(\mathcal{H} \rtimes \Gamma)} \mathcal{H} \rtimes \Gamma \cong C^{m e}(U) \otimes_{\mathbb{C}} \mathbb{C}\left[W_{0}^{\prime}\right] .
\end{aligned}
$$

The isomorphisms on the right-hand side are in the category of topological vector spaces, the algebra structure on the left-hand side is determined by

$$
\left(f_{1} \otimes h_{1}\right)\left(f_{2} \otimes h_{2}\right)=f_{1} f_{2} \otimes h_{1} h_{2} \quad f_{i} \in C^{m e}(U)^{W_{0}^{\prime}}, h_{i} \in \mathcal{H} \rtimes \Gamma .
$$

By Opd2, Proposition 4.5] $Z\left(\mathcal{H}^{a n}(U) \rtimes \Gamma\right) \cong C^{a n}(U)^{W_{0}^{\prime}}$, and similarly with meromorphic functions. 
For any $T^{\prime} \subset T$, let $\operatorname{Mod}_{f, T^{\prime}}(\mathcal{H} \rtimes \Gamma)$ be the category of finite dimensional $\mathcal{H} \rtimes \Gamma$ modules all whose $\mathcal{A}$-weights lie in $T^{\prime}$. By $\mathrm{Opd} 2$, Proposition 4.3] $\operatorname{Mod}_{f}\left(\mathcal{H}^{a n}(U) \rtimes\right.$ $\Gamma)$ is naturally equivalent to $\operatorname{Mod}_{f, U}(\mathcal{H} \rtimes \Gamma)$. (On the other hand, $\mathcal{H}^{m e}(U) \rtimes \Gamma$ does not have any nonzero finite dimensional representations over $\mathbb{C}$.)

Of course graded Hecke algebras can localized in exactly the same way, and the resulting algebras have analogous properties. By (1.14) the center of the algebra $\mathbb{H} \rtimes \Gamma$ is isomorphic to $\mathcal{O}\left(\mathfrak{t} / W_{0}^{\prime}\right)=\mathcal{O}(\mathfrak{t})^{W_{0}^{\prime}}$. For nonempty open $W_{0}^{\prime}$-invariant subsets $V$ of $\mathfrak{t}$ we get the algebras

$$
\begin{aligned}
& \mathbb{H}^{a n}(V) \rtimes \Gamma:=C^{a n}(V)^{W_{0}^{\prime}} \otimes_{Z(\mathbb{H} \rtimes \Gamma)} \mathbb{H} \rtimes \Gamma \cong C^{a n}(V) \otimes_{\mathbb{C}} \mathbb{C}\left[W_{0}^{\prime}\right], \\
& \mathbb{H}^{m e}(V) \rtimes \Gamma:=C^{m e}(V)^{W_{0}^{\prime}} \otimes_{Z(\mathbb{H} \rtimes \Gamma)} \mathbb{H} \rtimes \Gamma \cong C^{m e}(V) \otimes_{\mathbb{C}} \mathbb{C}\left[W_{0}^{\prime}\right] .
\end{aligned}
$$

Let $\mathbb{C}\left(T / W_{0}^{\prime}\right)$ be the quotient field of $Z(\mathcal{H} \rtimes \Gamma) \cong \mathcal{O}\left(T / W_{0}^{\prime}\right)$ and consider

$$
\mathbb{C}\left(T / W_{0}^{\prime}\right) \otimes_{Z(\mathcal{H} \rtimes \Gamma)} \mathcal{H} \rtimes \Gamma .
$$

As a vector space this is $\mathbb{C}(T) \otimes_{\mathcal{A}} \mathcal{H} \rtimes \Gamma \cong \mathbb{C}(T) \otimes_{\mathbb{C}} \mathbb{C}\left[W_{0}^{\prime}\right]$, while its multiplication is given by (1.23). Similarly, we let $\mathbb{C}\left(\mathfrak{t} / W_{0}^{\prime}\right)$ be the quotient field of $\mathcal{O}\left(\mathfrak{t} / W_{0}^{\prime}\right)$ and we construct the algebra

$$
\mathbb{C}\left(\mathfrak{t} / W_{0}^{\prime}\right) \otimes_{Z(\mathbb{H} \rtimes \Gamma)} \mathbb{H} \rtimes \Gamma .
$$

Its underlying vector space is

$$
\mathbb{C}(\mathfrak{t}) \otimes_{\mathcal{O}(\mathfrak{t})} \mathbb{H} \rtimes \Gamma \cong \mathbb{C}(\mathfrak{t}) \otimes_{\mathbb{C}} \mathbb{C}\left[W_{0}^{\prime}\right],
$$

and its multiplication is the obvious analogue of (1.23). Given a simple root $\alpha \in F_{0}$ we define elements $\imath_{s_{\alpha}}^{0} \in \mathbb{C}\left(T / W_{0}\right) \otimes_{Z(\mathcal{H})} \mathcal{H}$ and $\tilde{\imath}_{s_{\alpha}} \in \mathbb{C}\left(\mathfrak{t} / W_{0}\right) \otimes_{Z(\mathbb{H})} \mathbb{H}$ by

$$
\begin{aligned}
q\left(s_{\alpha}\right) c_{\alpha}\left(1+\imath_{s_{\alpha}}^{o}\right) & =1+q\left(s_{\alpha}\right)^{1 / 2} N_{s_{\alpha}}, \\
\tilde{c}_{\alpha}\left(1+\tilde{\imath}_{s_{\alpha}}\right) & =1+s_{\alpha} .
\end{aligned}
$$

Proposition 1.5.1. The elements $\imath_{s_{\alpha}}^{0}$ and $\tilde{\imath}_{s_{\alpha}}$ have the following properties:

(a) The map $s_{\alpha} \mapsto \imath_{s_{\alpha}}^{0}$ (respectively $s_{\alpha} \mapsto \tilde{\imath}_{s_{\alpha}}$ ) extends to a group homomorphism from $W_{0}^{\prime}$ to the multiplicative group of $\mathbb{C}\left(T / W_{0}^{\prime}\right) \otimes_{Z(\mathcal{H} \rtimes \Gamma)} \mathcal{H} \rtimes \Gamma$ (respectively $\left.\mathbb{C}\left(\mathfrak{t} / W_{0}^{\prime}\right) \otimes_{Z(\mathbb{H} \rtimes \Gamma)} \mathbb{H} \rtimes \Gamma\right)$.

(b) For $w \in W_{0}^{\prime}$ and $f \in \mathbb{C}(T) \cong \mathbb{C}\left(T / W_{0}^{\prime}\right) \otimes_{\mathcal{O}\left(T / W_{0}^{\prime}\right)} \mathcal{A}$ (respectively $\tilde{f} \in \mathbb{C}(\mathfrak{t})$ ) we have $\imath_{w}^{0} f \imath_{w^{-1}}^{0}=w(f)$ (respectively $\tilde{\imath}_{w} \tilde{f} \tilde{\imath}_{w^{-1}}=w(\tilde{f})$ ).

(c) The maps

$$
\begin{aligned}
& \mathbb{C}(T) \rtimes W_{0}^{\prime} \rightarrow \mathbb{C}\left(T / W_{0}^{\prime}\right) \otimes_{Z(\mathcal{H} \rtimes \Gamma)} \mathcal{H} \rtimes \Gamma: f w \quad \mapsto \quad f \imath_{w}^{0}, \\
& \mathbb{C}(\mathfrak{t}) \rtimes W_{0}^{\prime} \rightarrow \mathbb{C}\left(\mathfrak{t} / W_{0}^{\prime}\right) \otimes_{Z(\mathbb{H} \rtimes \Gamma)} \mathbb{H} \rtimes \Gamma: \tilde{f} w \quad \mapsto \quad \tilde{f} \tilde{\imath}_{w},
\end{aligned}
$$

are algebra isomorphisms.

(d) Let $P \subset F_{0}$ and $\gamma \in W_{0}^{\prime}$ be such that $\gamma(P) \subset F_{0}$. The automorphisms $\psi_{\gamma}$ from (1.17) satisfy

$$
\begin{array}{ll}
\psi_{\gamma}(h)=\imath_{\gamma}^{0} h \imath_{\gamma^{-1}}^{0} & \\
\psi_{\gamma}(\tilde{h})=\tilde{\imath}_{\gamma} \tilde{h} \tilde{\imath}_{\gamma^{-1}} & \tilde{h} \in \mathcal{H}^{P} \text { or } h \in \mathcal{H}_{P},
\end{array}
$$

Proof. (a), (b) and (c) with $W_{0}$ instead of $W_{0}^{\prime}$ can be found in Lus6, Section 5]. Notice that Lusztig calls these elements $\tau_{w}$ and $\tilde{\tau}_{w}$, while we follow the notation of Opd2, Section 4]. We extend this to $W_{0}^{\prime}$ by defining, for $\gamma \in \Gamma$ and $w \in W_{0}$ :

$$
\imath_{\gamma w}^{0}:=\gamma \imath_{w}^{0} \quad \text { and } \quad \tilde{\imath}_{\gamma w}=\gamma \tilde{\imath}_{w} .
$$

For (d) see [Lus6, Section 8] or [Sol5, Lemma 3.2]. 
We remark that by construction all the $\imath_{w}^{0}$ lie in the subalgebra $\mathbb{C}\left(T / T^{F_{0}}\right) \mathcal{H}\left(W_{0}, q\right)$ $\rtimes \Gamma$ and that the $\tilde{\imath}_{w}$ lie in the subalgebra $\mathbb{C}\left(\mathfrak{t} / \mathfrak{t}^{F_{0}}\right) \mathbb{C}\left[W_{0}^{\prime}\right]$. As was noticed in Opd2, Theorem 4.6], Proposition 1.5.1 can easily be generalized:

Corollary 1.5.2. Proposition 1.5 .1 remains valid under any of the following replacements:

- $\mathbb{C}(T)$ by $C^{m e}(U)$ or, if all the functions $c_{\alpha}$ are invertible on $U$, by $C^{a n}(U)$;

- $\mathbb{C}(\mathfrak{t})$ by $C^{m e}(V)$ or, if all the functions $\tilde{c}_{\alpha}$ are invertible on $V$, by $C^{a n}(V)$.

In particular,

$$
C^{m e}(U) \rtimes W_{0}^{\prime} \rightarrow C^{m e}(U)^{W_{0}^{\prime}} \otimes_{\mathcal{A}^{W_{0}^{\prime}}} \mathcal{H} \rtimes \Gamma: f w \rightarrow f \imath_{w}^{o}
$$

is an isomorphism of topological algebras.

1.6. The relation with reductive $p$-adic groups. Here we discuss how affine Hecke algebras arise in the representation theory of reductive $p$-adic groups. This section is rather sketchy, it mainly serves to provide some motivation and to prepare for our treatment of the Aubert-Baum-Plymen conjecture in Section 5.4. The main sources for this section are [BeDe, BeRu, Roc2, Hei, IwMa, Mor].

Let $\mathbb{F}$ be a nonarchimedean local field with a finite residue field. Let $\mathbf{G}$ be a connected reductive algebraic group defined over $\mathbb{F}$ and let $G=\mathbf{G}(\mathbb{F})$ be its group of $\mathbb{F}$-rational points. One briefly calls $G$ a reductive $p$-adic group, even though the characteristic of $\mathbb{F}$ is allowed to be positive.

An important theme, especially in relation with the arithmetic Langlands program, is the study of the category $\operatorname{Mod}(G)$ of smooth $G$-representations on complex vector spaces. A first step to simplify this problem is the Bernstein decomposition, which we recall now. Let $P$ be a parabolic subgroup of $G$ and $M$ a Levi subgroup of $P$. Although $G$ and $M$ are unimodular, the modular function $\delta_{P}$ of $P$ is in general not constant. Let $\sigma$ be an irreducible supercuspidal representation of $M$. In this situation we call $(M, \sigma)$ a cuspidal pair, and with parabolic induction we construct the $G$-representation

$$
I_{P}^{G}(\sigma):=\operatorname{Ind}_{P}^{G}\left(\delta_{P}^{1 / 2} \otimes \sigma\right) .
$$

This means that first we inflate $\sigma$ to $P$ and then we apply the normalized smooth induction functor. The normalization refers to the twist by $\delta_{P}^{1 / 2}$, which is useful to preserve unitarity. Let $\operatorname{Irr}(G)$ be the collection of irreducible representations in $\operatorname{Mod}(G)$, modulo equivalence. For every $\pi \in \operatorname{Irr}(G)$ there is a cuspidal pair $(M, \sigma)$, uniquely determined up to $G$-conjugacy, such that $\pi$ is a subquotient of $I_{P}^{G}(\sigma)$.

Let $G^{0}$ be the normal subgroup of $G$ generated by all compact subgroups. Recall that a character $\chi: G \rightarrow \mathbb{C}^{\times}$is called unramified if its kernel contains $G^{0}$. The group $X_{u r}(G)$ of unramified characters forms a complex algebraic torus whose character lattice is naturally isomorphic to the lattice $X^{*}(G)$ of algebraic characters $G \rightarrow \mathbb{F}^{\times}$. We say that two cuspidal pairs $(M, \sigma)$ and $\left(M^{\prime}, \sigma^{\prime}\right)$ are inertially equivalent if there exist $g \in G$ and $\chi^{\prime} \in X_{u r}\left(M^{\prime}\right)$ such that

$$
M^{\prime}=g M g^{-1} \quad \text { and } \quad \sigma^{\prime} \otimes \chi^{\prime} \cong \sigma^{g},
$$

where $\sigma^{g}\left(m^{\prime}\right)=\sigma\left(g^{-1} m^{\prime} g\right)$. With an inertial equivalence class $\mathfrak{s}=[M, \sigma]_{G}$ one associates a full subcategory $\operatorname{Mod}_{\mathfrak{s}}(G)$ of $\operatorname{Mod}(G)$. Its objects are by definition those smooth representations $\pi$ with the property that for every irreducible subquotient $\rho$ of $\pi$ there is a $(M, \sigma) \in \mathfrak{s}$ such that $\rho$ is a subrepresentation of $I_{P}^{G}(\sigma)$. The collection $\mathfrak{B}(G)$ of inertial equivalence classes is countably infinite (unless $G=\{1\}$ ). 
The Bernstein decomposition [BeDe, Proposition 2.10] states that

$$
\operatorname{Mod}(G)=\prod_{\mathfrak{s} \in \mathfrak{B}(G)} \operatorname{Mod}_{\mathfrak{s}}(G),
$$

a direct product of categories. The subcategories $\operatorname{Mod}_{\mathfrak{s}}(G)$ (or rather their subsets of irreducible representations) are also called the Bernstein components of the smooth dual of $G$.

The Hecke algebra $\mathcal{H}(G)$ is the vector space of locally constant, compactly supported functions on $G$, endowed with the convolution product. $\operatorname{Mod}(G)$ is naturally equivalent to the category $\operatorname{Mod}(\mathcal{H}(G)$ ) of essential $\mathcal{H}(G)$-modules. (A module $V$ is called essential if $\mathcal{H}(G) V=V$, which is not automatic because $\mathcal{H}(G)$ does not have a unit.) Corresponding to (1.27) there is a decomposition

$$
\mathcal{H}(G)=\bigoplus_{\mathfrak{s} \in \mathfrak{B}(G)} \mathcal{H}(G)_{\mathfrak{s}}
$$

of the Hecke algebra of $G$ into two-sided ideals. In several cases $\mathcal{H}(G)_{\mathfrak{s}}$ is known to be Morita equivalent to an affine Hecke algebra.

In the classical case $I \mathrm{wMa}$, Bor $G$ is split and $\operatorname{Mod}_{\mathfrak{s}}(G)$ is the category of Iwahori spherical representations. That is, those smooth $G$-representations $V$ that are generated by $V^{I}$, where $I$ is an Iwahori subgroup of $G$. Then $\mathcal{H}(G)_{\mathfrak{s}}$ is Morita equivalent to the algebra $\mathcal{H}(G, I)$ of $I$-bi-invariant functions in $\mathcal{H}(G)$, and $\mathcal{H}(G, I)$ is isomorphic to an affine Hecke algebra $\mathcal{H}(\mathcal{R}, q)$. The root datum $\mathcal{R}=\left(X, R_{0}, Y, R_{0}^{\vee}, F_{0}\right)$ is dual to the root datum of $(\mathbf{G}, \mathbf{T})$, where $\mathbf{T}(\mathbb{F})$ is a split maximal torus of $G=\mathbf{G}(\mathbb{F})$. More explicitly:

- $X$ is the cocharacter lattice of $\mathbf{T}$;

- $Y$ is the character lattice of $\mathbf{T}$;

- $R_{0}^{\vee}$ is the root system of $(\mathbf{G}, \mathbf{T})$;

- $R_{0}$ is the set coroots of $(\mathbf{G}, \mathbf{T})$;

- $F_{0}$ and $F_{0}^{\vee}$ are determined by $I$;

- $q$ is the cardinality of the residue field of $\mathbb{F}$.

For a general inertial equivalence class $\mathfrak{s}=[M, \sigma]_{G}$ it is expected that $\mathcal{H}(G)_{\mathfrak{s}}$ is Morita equivalent to an affine Hecke algebra or to a closely related kind of algebra. We discuss some ingredients of this conjectural relation between the representation theory of reductive $p$-adic groups and that of affine Hecke algebras.

Let $\sigma^{0}$ be an irreducible subrepresentation of $\left.\sigma\right|_{M^{0}}$ and define $\Sigma=\operatorname{ind}_{M^{0}}^{M}\left(\sigma^{0}\right)$. According to $\mathrm{BeRu}$, Theorem 23] $I_{P}^{G}(\Sigma)$ is a finitely generated projective generator of the category $\operatorname{Mod}_{\mathfrak{s}}(G)$. By $\operatorname{BeRu}$, Lemma 22] $\operatorname{Mod}_{\mathfrak{s}}(G)=\operatorname{Mod}\left(\mathcal{H}(G)_{\mathfrak{s}}\right)$ is naturally equivalent to the category of right $\operatorname{End}_{G}\left(I_{P}^{G}(\Sigma)\right)$-modules. So if $\operatorname{End}_{G}\left(I_{P}^{G}(\Sigma)\right)$ would be isomorphic to its opposite algebra (which is likely), then it is Morita equivalent to $\mathcal{H}(G)_{\mathfrak{s}}$.

Let us describe the center of $\operatorname{End}_{G}\left(I_{P}^{G}(\Sigma)\right)$. The map

$$
X_{u r}(M) \rightarrow \operatorname{Irr}_{[M, \sigma]_{M}}(M): \chi \mapsto \chi \otimes \sigma
$$

is surjective and its fibers are cosets of a finite subgroup $\operatorname{Stab}(\sigma) \subset X_{u r}(M)$. Let

$$
M_{\sigma}:=\bigcap_{\chi \in \operatorname{Stab}(\sigma)} \operatorname{ker}(\chi) \subset M .
$$

Roche [Roc2, Proposition 5.3] showed that $\operatorname{End}_{M}(\Sigma)$ is a free $\mathcal{O}\left(X_{u r}\left(M_{\sigma}\right)\right)$-module of rank $m^{2}$, where $m$ is the multiplicity of $\sigma^{0}$ in $\left.\sigma\right|_{M^{0}}$. Moreover, the center of 
$\operatorname{End}_{M}(\Sigma)$ is isomorphic to $\mathcal{O}\left(X_{u r}\left(M_{\sigma}\right)\right)$, and $\operatorname{End}_{M}(\Sigma)$ embeds in $\operatorname{End}_{G}\left(I_{P}^{G}(\Sigma)\right)$ by functoriality. The group

$$
N_{G}(M, \sigma):=\left\{g \in G: g M g^{-1}=M, \sigma^{g} \cong \chi \otimes \sigma \text { for some } \chi \in X_{u r}(M)\right\}
$$

acts on the family of representations $\left\{I_{P}^{G}(\chi \otimes \Sigma): \chi \in X_{u r}(M)\right\}$, and via this on $X_{u r}\left(M_{\sigma}\right)$. The subgroup $M \subset N_{G}(M, \sigma)$ acts trivially, so we get an action of the finite group

$$
W_{\sigma}:=N_{G}(M, \sigma) / M
$$

According to BeDe, Théorème 2.13], the center of $\operatorname{End}_{G}\left(I_{P}^{G}(\Sigma)\right)$ is isomorphic to $\mathcal{O}\left(X_{u r}\left(M_{\sigma}\right)\right)^{W_{\sigma}}=\mathcal{O}\left(X_{u r}\left(M_{\sigma}\right) / W_{\sigma}\right)$. By $\left[\underline{\operatorname{Roc} 2}\right.$, Lemma 7.3] $\operatorname{End}_{G}\left(I_{P}^{G}(\Sigma)\right)$ is a free $\operatorname{End}_{M}(\Sigma)$-module of rank $\left|W_{\sigma}\right|$.

Next we indicate how to associate a root datum to $\operatorname{End}_{G}\left(I_{P}^{G}(\Sigma)\right)$. See Hei, Section 6] for more details in the case of classical groups. Let $A$ be the maximal split torus of $Z(M)$, let $X^{*}(A)$ be its character lattice and $X_{*}(A)$ its cocharacter lattice. There are natural isomorphisms,

$$
X_{u r}(M) \cong X^{*}(A) \otimes_{\mathbb{Z}} \mathbb{C}^{\times} \cong \operatorname{Hom}\left(X_{*}(A), \mathbb{C}^{\times}\right) .
$$

In $X^{*}(A)$ we have the root system $R(G, A)$ and in $X_{*}(A)$ we have the set $R^{\vee}(G, A)$ of coroots of $(G, A)$. The parabolic subgroup $P$ determines positive systems $R(P, A)$ and $R^{\vee}(P, A)$. Altogether we constructed a (nonreduced) based root datum,

$$
\mathcal{R}_{M}:=\left(X_{*}(A), R^{\vee}(G, A), X^{*}(A), R(G, A), R^{\vee}(P, A)\right),
$$

from which one can of course deduce a reduced based root datum.

Yet $\mathcal{R}_{M}$ is not good enough, it does not take $\sigma$ into account. Put

$$
\begin{aligned}
& X_{\sigma}:=\operatorname{Hom}\left(X_{u r}\left(M_{\sigma}\right), \mathbb{C}^{\times}\right) \cong \operatorname{Hom}\left(X_{u r}\left(M_{\sigma} \cap A\right), \mathbb{C}^{\times}\right) \subset X_{*}(A), \\
& Y_{\sigma}:=\operatorname{Hom}\left(X_{\sigma}, \mathbb{Z}\right) \cong X^{*}\left(M_{\sigma} \cap A\right) \cong X^{*}(A) \text {. }
\end{aligned}
$$

Assume for simplicity that $\left.\sigma\right|_{M^{0}}$ is multiplicity free, or equivalently that $\operatorname{End}_{M}(\Sigma)=$ $\mathcal{O}\left(X_{u r}\left(M_{\sigma}\right)\right)$. Then the above says that $\operatorname{End}_{G}\left(I_{P}^{G}(\Sigma)\right)$ is a free module of rank $\left|W_{\sigma}\right|$ over $\mathbb{C}\left[X_{\sigma}\right]$. We want to associate a root system to $W_{\sigma}$. In general, $W_{\sigma}$ does not contain $W(G, A)=N_{G}(M) / M$, so we have to replace $R(G, A)$ by

$$
R_{\sigma, n r}:=\left\{\alpha \in R(G, A): s_{\alpha} \in W_{\sigma}\right\},
$$

and $R^{\vee}(G, A)$ by $R_{\sigma, n r}^{\vee}$. Let $R_{\sigma}^{\vee}$ be the reduced root system of $R_{\sigma, n r}^{\vee}$ and $R_{\sigma}$ the dual root system, which consists of the nonmultipliable roots in $R_{\sigma, n r}$. Let $F_{\sigma}$ be the unique basis of $R_{\sigma}$ contained in $R(P, A)$. Then $W\left(R_{\sigma}\right)$ is a normal subgroup of $W_{\sigma}$ and

$$
W_{\sigma} \cong W\left(R_{\sigma}\right) \rtimes \Gamma_{\sigma} \quad \text { where } \quad \Gamma_{\sigma}=\left\{w \in W_{\sigma}: w\left(F_{\sigma}\right)=F_{\sigma}\right\} .
$$

As $\sigma$ is not explicit, it is difficult to say which diagram automorphism groups $\Gamma_{\sigma}$ can occur here. A priori there does not seem to be any particular restriction.

All this suggests that, if $\operatorname{End}_{G}\left(I_{P}^{G}(\Sigma)\right)$ is isomorphic to some affine Hecke algebra, then to

$$
\mathcal{H}_{\sigma} \rtimes \Gamma_{\sigma}=\mathcal{H}\left(\mathcal{R}_{\sigma}, q_{\sigma}\right) \rtimes \Gamma_{\sigma}:=\mathcal{H}\left(X_{\sigma}, R_{\sigma}^{\vee}, Y_{\sigma}, R_{\sigma}, F_{\sigma}^{\vee}, q_{\sigma}\right) \rtimes \Gamma_{\sigma} .
$$

In fact, it also possible that the $\Gamma_{\sigma}$-action is twisted by a cocycle [Mor, Section 7], but we ignore this subtlety here. We note that little would change upon replacing $G$ by a disconnected group, that would only lead to a larger group of diagram automorphisms. 
We note that this description of $\operatorname{End}_{G}\left(I_{P}^{G}(\Sigma)\right)$ is compatible with parabolic induction. Every parabolic subgroup $Q \subset G$ containing $P$ gives rise to a subalgebra

$$
\operatorname{End}_{Q}\left(I_{P}^{Q}(\Sigma)\right) \subset \operatorname{End}_{G}\left(I_{P}^{G}(\Sigma)\right),
$$

which via (1.28) and

$$
R_{\sigma, n r}^{Q}=R(Q, A) \subset R(P, A)=R_{\sigma, n r}
$$

corresponds to a parabolic subalgebra $\mathcal{H}_{\sigma}^{Q} \rtimes \Gamma_{\sigma, Q} \subset \mathcal{H}_{\sigma} \rtimes \Gamma_{\sigma}$.

By analogy with the Iwahori case the numbers $q_{\sigma}(w)$ are related to the affine Coxeter complex of $X_{*}(A) \rtimes W\left(R^{\vee}(G, A)\right)$. After fixing a fundamental chamber $C_{0}$, every $w \in X_{*}(A) \rtimes W_{\sigma}$ determines a chamber $w\left(C_{0}\right)$. This affine Coxeter complex can be regarded as a subset of the Bruhat-Tits building of $G$, so $C_{0}$ has a stabilizer $K \subset G$. In view of [Mor, Section 6], a good candidate for $q_{\sigma}(w)$ is $[K w K: K]$. In particular, for a simple reflection $s_{\alpha} \in W\left(R_{\sigma}^{\vee}\right)$ this works out to $q_{\sigma}\left(s_{\alpha}\right)=q^{d_{\alpha}}$, where $q$ is cardinality of the residue field of $\mathbb{F}$ and $d_{\alpha}$ is the dimension of the $\alpha$ weight space in the $A$-representation $\operatorname{Lie}(G)$. Hence $q_{\sigma}$ is a positive parameter function and, if $A$ is not a split maximal torus of $G, q_{\sigma}$ tends to be nonconstant on the set of simple reflections.

As said before, most of the above is conjectural. The problem is that in general it is not known whether one can construct elements $N_{w}\left(w \in W_{\sigma}\right)$ that satisfy the multiplication rules of an extended affine Hecke algebra. To that end one has to study the intertwining operators between parabolically induced representations very carefully.

Let us list the cases in which it is proven that $\operatorname{End}_{G}\left(I_{P}^{G}(\Sigma)\right)$ is isomorphic to an (extended) affine Hecke algebra:

- $G$ split, $\mathfrak{s}$ the Iwahori-spherical component IwMa, Bor;

- $G=G L_{n}(\mathbb{F}) \mathfrak{s}$ arbitrary — from the work of Bushnell and Kutzko on types BuKu1, BuKu2, BuKu3;

- $G=S L_{n}(\mathbb{F})$, many $\mathfrak{s}$ GoRo (for general $\mathfrak{s}$ the Hecke algebra is known to have a closely related shape);

- $G$ a (special) orthogonal group, a symplectic group or an inner form of $G L_{n}(\mathbb{F}), \mathfrak{s}$ arbitrary [Hei];

- $G=G S p_{4}(\mathbb{F})$ or $G=U(2,1), \mathfrak{s}$ arbitrary Moy1, Moy2;

- $G$ classical, certain $\mathfrak{s}$ Kim1, Kim2, Blo;

- $G$ split (with mild restrictions on the residual characteristic), $\mathfrak{s}$ in the principal series [Roc1];

- $G$ arbitrary, $\sigma$ induced from a level 0 cuspidal representation of a parahoric subgroup of $G$ Mor, MoPr, Lus7;

Of course there is a lot of overlap in this list. For $G L_{n}, S L_{n}, G S p_{4}$ and $U(2,1)$ the above references do much more, they classify the smooth dual of $G$. In the level 0 case, Morris Mor showed that the parameters $q_{\alpha}$ are the same as those for analogous Hecke algebras of finite Chevalley groups. Those parameters were determined explicitly in Lus1, and often they are not equal on all simple roots.

Apart from this list, there are many inertial equivalence classes $\mathfrak{s}$ for which $\operatorname{End}_{G}\left(I_{P}^{G}(\Sigma)\right)$ is Morita equivalent to a commutative algebra. This is the case for supercuspidal $G$-representations $\sigma$ such that $\left.\sigma\right|_{G^{0}}$ is multiplicity-free, and more generally it tends to happen when $R_{\sigma}$ is empty. 


\section{Classification of irReducible Representations}

This section leads to the main result of the paper (Theorem 2.3.1). It is an affine analogue of the Springer correspondence. Together with Kazhdan-Lusztig-theory, the classical Springer correspondence parametrizes the irreducible representations of a finite Weyl group with certain representations of an affine Hecke algebra with equal parameters BaMo1. This correspondence is known to remain valid for affine or graded Hecke algebras with certain specific unequal parameters Ciu.

We construct a natural map from irreducible $\mathcal{H}$-representations to representations of the extended affine Weyl group $W$. Not all representations in the image are irreducible, but the image does form a $\mathbb{Q}$-basis of the representation ring of $W$.

The proof proceeds by reduction to a result that the author previously obtained for graded Hecke algebras [Sol6]. To carry out this reduction, we need variations on three well-known results in representation theory of Hecke algebras. The first two are due to Lusztig and allow one to descend from affine Hecke algebras to graded Hecke algebras. We adjust these results to make them more suitable for affine Hecke algebras with arbitrary positive parameters.

Third, there is the Langlands classification (Theorem 2.2.4), which comes from reductive groups and reduces the classification of irreducible representations to that of irreducible tempered ones. For affine Hecke algebras it did not appear in the literature before, although it was of course known to experts. Because we want to include diagram automorphisms in our affine Hecke algebras, we need a more refined version of the Langlands classification (Corollary 2.2.5). It turns out that one has to add an extra ingredient to the Langlands parameters, and that the unicity claim has to be changed accordingly.

However, these results do not suffice to complete the proof of Theorem 2.3.1 for nontempered representations, that will be done in the next chapter.

2.1. Two reduction theorems. The study of irreducible representations of $\mathcal{H} \rtimes \Gamma$ is simplified by two reduction theorems, which are essentially due to Lusztig Lus6]. The first one reduces to the case of modules whose central character is positive on the lattice $\mathbb{Z} R_{1}$. The second one relates these to modules of an associated graded Hecke algebra.

Given $t \in T$ and $\alpha \in R_{0}$, Lus6, Lemma 3.15] tells us that

$$
s_{\alpha}(t)=t \text { if and only if } \alpha(t)= \begin{cases}1 & \text { if } \alpha^{\vee} \notin 2 Y, \\ \pm 1 & \text { if } \alpha^{\vee} \in 2 Y .\end{cases}
$$

We define $R_{t}:=\left\{\alpha \in R_{0}: s_{\alpha}(t)=t\right\}$. The collection of long roots in $R_{t, n r}$ is $\left\{\beta \in R_{1}: \beta(t)=1\right\}$. Let $F_{t}$ be the unique basis of $R_{t}$ that is contained in $R_{0}^{+}$. Then

$$
W_{F_{t}, t}^{\prime}:=\left\{w \in W_{0} \rtimes \Gamma: w(t)=t, w\left(F_{t}\right)=F_{t}\right\}
$$

is a group of automorphisms of the Dynkin diagram of $\left(R_{t}, F_{t}\right)$. Moreover, the isotropy group of $t$ in $W_{0} \rtimes \Gamma$ is

$$
W_{t}^{\prime}=\left(W_{0} \rtimes \Gamma\right)_{t}=W\left(R_{t}\right) \rtimes W_{F_{t}, t}^{\prime} .
$$

We can define a parameter function $q_{t}$ for the based root datum

$$
\mathcal{R}_{t}:=\left(X, R_{t}, Y, R_{t}^{\vee}, F_{t}\right)
$$

via restriction from $R_{n r}^{\vee}$ to $R_{t, n r}^{\vee}$. 
Since $F_{t}$ does not have to be a subset of $F_{0}, \mathcal{R}_{t}$ does not always fit in the setting of Subsection 1.4, but this can be fixed without many problems. For $u \in T_{u n}$ we define

$$
P(u):=F_{0} \cap \mathbb{Q} R_{u} .
$$

Then $R_{P(u)}$ is a parabolic root subsystem of $R_{0}$ that contains $R_{u}$ as a subsystem of full rank. Although this definition would also make sense for general elements of $T$, we use it only for $T_{u n}$, to avoid a clash with the notation of [Opd2, Section 4.1]. We note that the lattice

$$
\mathbb{Z} P(u)=\mathbb{Z} R_{0} \cap \mathbb{Q} R_{u}
$$

can be strictly larger than $\mathbb{Z} R_{u}$.

To study $\mathcal{H}$-representations with central character $W_{0}^{\prime} u c$ we need a well-chosen neighborhood of $u c \in T_{u n} T_{r s}$.

Condition 2.1.1. Let $B \subset \mathfrak{t}$ be such that

(a) $B$ is an open ball centred around $0 \in \mathfrak{t}$;

(b) $\Im(\alpha(b))<\pi$ for all $\alpha \in R_{0}, b \in B$;

(c) $\exp : B \rightarrow \exp (B)$ is a diffeomorphism (this follows from (b) if $R_{0}$ spans $\left.\mathfrak{a}^{*}\right)$

(d) if $c_{\alpha}(t) \in\{0, \infty\}$ for some $\alpha \in R_{0}, t \in u c \exp \bar{B}$, then $c_{\alpha}(u c) \in\{0, \infty\}$;

(e) if $w \in W_{0}^{\prime}$ and $w(u c \exp \bar{B}) \cap u c \exp \bar{B} \neq \emptyset$, then $w(u c)=u c$.

Since $W_{0}^{\prime}$ acts isometrically on $\mathfrak{t}$, (a) implies that $B$ is $W_{0}^{\prime}$-invariant. There always exist balls satisfiying these conditions, and if we have one such $B$, then $\epsilon B$ with $\epsilon \in(0,1]$ also works.

We will phrase our first reduction theorem in such a way that it depends mainly on the unitary part of the central character, it will decompose a representation in subspaces corresponding to the points of the orbit $W_{0}^{\prime} u$. We note that $R_{u c} \subset R_{u}$ and $W_{u c}^{\prime} \subset W_{u}^{\prime}$. Given $B$ satisfying the above conditions, we define

$$
U=W_{0}^{\prime} u c \exp (B), U_{P(u)}=W_{\mathbb{Z} P(u)}^{\prime} u c \exp (B) \text { and } U_{u}=W_{u}^{\prime} u c \exp (B) .
$$

We are interested in the algebras $\mathcal{H}(\mathcal{R}, q)^{\text {an }}(U) \rtimes \Gamma, \mathcal{H}\left(\mathcal{R}^{P(u)}, q^{P(u)}\right)^{\text {an }}\left(U_{P(u)}\right) \rtimes$ $W_{P(u)}^{\prime}$ and $\mathcal{H}\left(\mathcal{R}_{u}, q_{u}\right)^{a n}\left(U_{u}\right) \rtimes W_{F_{u}, u}^{\prime}$. Their respective centers

$$
C^{a n}(U)^{W_{0}^{\prime}}, C^{a n}\left(U_{P(u)}\right)^{W_{\mathbb{Z} P(u)}^{\prime}} \text { and } C^{a n}\left(U_{u}\right)^{W_{u}^{\prime}}
$$

are naturally isomorphic, via the embeddings $U_{u} \subset U_{P(u)} \subset U$. For any subset $\varpi \subset W_{0}^{\prime} u c$ we define $1_{\varpi} \in C^{a n}(U)$ by

$$
1_{\varpi}(t)= \begin{cases}1 & \text { if } t \in \varpi \exp (B), \\ 0 & \text { if } t \in U \backslash \varpi \exp (B) .\end{cases}
$$

Theorem 2.1.2 (First reduction theorem).

(a) There are natural isomorphisms of $C^{\text {an }}(U)^{W_{0}^{\prime}}$-algebras

$$
\begin{array}{ll}
\mathcal{H}\left(\mathcal{R}^{P(u)}, q^{P(u)}\right)^{a n}\left(U_{P(u)}\right) \rtimes W_{P(u)}^{\prime} & \cong 1_{W_{\mathbb{Z} P(u)}^{\prime} u c}\left(\mathcal{H}^{a n}(U) \rtimes \Gamma\right) 1_{W_{\mathbb{Z} P(u)}^{\prime} u c}, \\
\left.\mathcal{H}\left(\mathcal{R}_{u}, q_{u}\right)^{a n}\left(U_{u}\right) \rtimes W_{F_{u}, u}^{\prime a n}(U) \rtimes \Gamma\right) 1_{W_{u}^{\prime} u c .} & \cong 1_{W_{u}^{\prime} u c}\left(\mathcal{H}^{a n}(U)\right.
\end{array}
$$

(b) These can be extended (not naturally) to isomorphisms of $C^{a n}(U)^{W_{0}^{\prime}}$-algebras

$$
\begin{aligned}
& \mathcal{H}^{a n}(U) \rtimes \Gamma \cong M_{\left[W_{0}^{\prime}: W_{\mathbb{Z} P(u)}^{\prime}\right.}\left(1_{W_{\mathbb{Z} P(u)}^{\prime} u c}\left(\mathcal{H}^{a n}(U) \rtimes \Gamma\right) 1_{W_{\mathbb{Z} P(u)}^{\prime} u c}\right), \\
& \mathcal{H}^{a n}(U) \rtimes \Gamma \cong M_{\left[W_{0}^{\prime}: W_{u}^{\prime}\right]}\left(1_{W_{u}^{\prime} u c}\left(\mathcal{H}^{a n}(U) \rtimes \Gamma\right) 1_{W_{u}^{\prime} u c}\right),
\end{aligned}
$$


where $M_{n}(A)$ denotes the algebra of $n \times n$-matrices with coefficients in an algebra $A$.

(c) The following maps are natural equivalences of categories:

$$
\begin{aligned}
& \operatorname{Mod}_{f, U}(\mathcal{H}(\mathcal{R}, q) \rtimes \Gamma) \leftrightarrow \operatorname{Mod}_{f, U_{P(u)}}\left(\mathcal{H}^{P(u)} \rtimes W_{P(u)}^{\prime}\right) \leftrightarrow \operatorname{Mod}_{f, U_{u}}\left(\mathcal{H}\left(\mathcal{R}_{u}, q_{u}\right) \rtimes W_{F_{u}, u}^{\prime}\right), \\
& V \mapsto 1_{W_{Z P(u)}^{\prime} u c} V \mapsto 1_{W_{u}^{\prime} u c} V, \\
& \operatorname{Ind}_{\mathcal{H}\left(\mathcal{R}_{u}, q_{u}\right) \rtimes W_{F_{u}, u}^{\prime}}^{\mathcal{H} \rtimes \Gamma}(\pi) \longleftrightarrow \operatorname{Ind}_{\mathcal{H}\left(\mathcal{R}_{u}, q_{u}\right) \rtimes W_{F_{u}, u}^{\prime}}^{\mathcal{H}^{P(u)} \rtimes W_{P(u)}^{\prime}}(\pi) \leftrightarrow \pi .
\end{aligned}
$$

Proof. (a) This is a variation on Opd2, Theorem 4.10], which itself varied on [Lus6, Theorem 8.6]. Compared to Lusztig we replaced $Y \otimes\left\langle v_{0}\right\rangle \subset T$ by $T_{r s}=Y \otimes \mathbb{R}_{>0}$, we substituted his $R_{t}$ by a larger root system, we added the group $\Gamma$ and we localized with analytic functions instead of formal completions at one central character.

The first change is allowed because we localize only on $T$, and not simultaneously in the $q$-direction like Lusztig. Hence the structure of the subgroup $\left\langle v_{0}\right\rangle \subset \mathbb{C}^{\times}$ becomes irrelevant to the arguments of [Lus6, Section 8]. With this modification Lusztig's $R_{t}$ becomes our $R_{u}$ and his equivalence classes $c \in \mathcal{P}$ correspond to the orbits $W_{u}^{\prime} u c \subset W_{0}^{\prime} u c$. The root system $R_{P(u)}$ and the orbits $W_{\mathbb{Z} P(u)}^{\prime} u c \subset W_{0}^{\prime} u c$ fall into the same framework, so the constructions from [Lus6, Section 8] can still be carried out. Since $\mathbb{Z} R_{u} \subset \mathbb{Z} R_{P(u)}$ there are fewer orbits in the second case, so it actually becomes somewhat easier.

By [Lus6, Lemma 8.13.b] Lusztig's version of the isomorphisms (a) sends $\gamma \in \Gamma(c)$ to $1_{c} \imath_{\gamma}^{0} 1_{c}$, for some $c \in \mathcal{P}$. Translated to our setting this means that we can include the appropriate diagram automorphisms by defining

$$
\begin{array}{ccccc}
W_{P(u)}^{\prime} & \ni & \gamma & 1_{W_{\mathbb{Z} P(u)}^{\prime} u c} l_{\gamma}^{0} 1_{W_{\mathbb{Z} P(u)}^{\prime} u c}, \\
W_{F_{u}, u}^{\prime} & \ni & \gamma & 1_{W_{u}^{\prime} u c} l_{\gamma}^{0} 1_{W_{u}^{\prime} u c}
\end{array}
$$

Finally, that Lusztig's arguments also apply with analytic localization was already checked by Opdam Opd2, Section 4.1].

(b) Knowing (a), this can proved just as in [Lus6, 4.16].

(c) By Opd2, Proposition 4.3] the categories in the statement are the categories of finite dimensional modules of the algebras figuring in (a) and (b). Therefore the maps in (c) are just the standard equivalences between the module categories of $B$ and $M_{n}(B)$, translated with (a) and (b).

Remark 2.1.3. This reduction theorem more or less forces one to consider diagram automorphisms: the groups $W_{\mathbb{Z} P(u)}^{\prime}$ and $W_{F_{u}, u}^{\prime}$ can be nontrivial even if $\Gamma=\{\mathrm{id}\}$.

The notation with induction functors in part (c) is a little sloppy, since $W_{F_{u}, u}^{\prime}$ need not be contained in $\Gamma$ or in $W_{\mathbb{Z} P(u)}^{\prime}$. In such cases these induction functors are defined via part (a).

The first reduction theorem also enables us to make sense of $\operatorname{Ind}_{\mathcal{H}\left(\mathcal{R}^{P}, q^{P}\right) \rtimes \Gamma_{P}^{\prime}}^{\mathcal{H} x}$ for any $P \subset F_{0}$ and any subgroup $\Gamma_{P}^{\prime} \subset W_{P}^{\prime}$. Namely, first induce from $\mathcal{H}\left(\mathcal{R}^{P}, q^{P}\right) \rtimes \Gamma_{P}^{\prime}$ to $\mathcal{H}\left(\mathcal{R}^{P}, q^{P}\right) \rtimes W_{\mathbb{Z} P}^{\prime}$, then choose $u \in T_{u n}$ such that $P(u)=P$ and finally use (c).

By (2.1) we have $\alpha(u)=1$ for all $\alpha \in R_{1} \cap \mathbb{Q} R_{t}$, so $\alpha(t)=\alpha(u) \alpha(c)>0$ for such roots. By definition $u$ is fixed by $W_{u}^{\prime}$, so Theorem 2.1.2 allows us to restrict our attention to $\mathcal{H} \rtimes \Gamma$-modules whose central character is positive on the sublattice $\mathbb{Z} R_{1} \subseteq X$. 
Next we want to reduce to graded Hecke algebras. We define a parameter function $k_{u}$ for the degenerate root datum $\tilde{\mathcal{R}}_{u}=\left(\mathfrak{a}, R_{u}, \mathfrak{a}^{*}, R_{u}^{\vee}, F_{u}\right)$ by

$$
k_{u, \alpha}=\left(\log q\left(s_{\alpha}\right)+\alpha(u) \log q\left(t_{\alpha} s_{\alpha}\right)\right) / 2 .
$$

Recall that $\alpha \in R_{u}$ implies that $s_{\alpha}(u)=u$ and $\alpha(u)^{2}=1$. We will see in (4.4) that for this choice of $k_{u}$ the function $\tilde{c}_{\alpha}$ can be regarded as the first order approximation of $q\left(s_{\alpha}\right) c_{\alpha}$ in a neighborhood of $q=1$ and $u \in T$.

Let us pick $u \in T_{u n}^{W_{0}^{\prime}}$, so $\alpha(u)= \pm 1$ for all $\alpha \in R_{0}$. Then the map

$$
\exp _{u}: \mathfrak{t} \rightarrow T, \lambda \mapsto u \exp (\lambda)
$$

is $W_{0}^{\prime}$-equivariant. To find a relation between $\mathcal{H}(\mathcal{R}, q) \rtimes \Gamma$ and $\mathbb{H}\left(\tilde{\mathcal{R}}_{u}, k_{u}\right) \rtimes \Gamma$, we first extend these algebras with analytic localization. For every open nonempty $W_{0}^{\prime}$-invariant $V \subset \mathfrak{t}$ we can define an algebra homomorphism

$$
\begin{aligned}
\Phi_{u}: \mathcal{H}^{m e}\left(\exp _{u}(V)\right) \rtimes \Gamma & \rightarrow \mathbb{H}\left(\tilde{\mathcal{R}}_{u}, k_{u}\right)^{m e}(V) \rtimes \Gamma, \\
f \imath_{w}^{0} & \mapsto\left(f \circ \exp _{u}\right) \tilde{\imath}_{w} .
\end{aligned}
$$

Theorem 2.1.4 (Second reduction theorem). Let $u \in T_{u n}^{W_{0}^{\prime}}$ and let $V$ be as above, such that, moreover, $\exp _{u}$ is injective on $V$.

(a) The map $\exp _{u}$ induces an isomorphism $C^{a n}\left(\exp _{u}(V)\right)^{W_{0}^{\prime}} \rightarrow C^{a n}(V)^{W_{0}^{\prime}}$.

(b) Suppose that every $\lambda \in V$ satisfies

$$
\begin{aligned}
& \langle\alpha, \lambda\rangle,\langle\alpha, \lambda\rangle+k_{u, \alpha} \quad \notin 2 \pi i \mathbb{Z} \backslash\{0\} \quad \text { for } \alpha \in R_{0} \cap R_{1}, \\
& \langle\alpha, \lambda\rangle,\langle\alpha, \lambda\rangle+k_{u, \alpha} \quad \notin \quad \pi i \mathbb{Z} \backslash\{0\} \quad \text { for } \alpha \in R_{0} \backslash R_{1} \text {. }
\end{aligned}
$$

Then $\Phi_{u}$ restricts to an isomorphism of $C^{a n}(V)^{W_{0}^{\prime}}$-algebras

$$
\Phi_{u}: \mathcal{H}^{a n}\left(\exp _{u}(V)\right) \rtimes \Gamma \rightarrow \mathbb{H}\left(\tilde{\mathcal{R}}_{u}, k_{u}\right)^{a n}(V) \rtimes \Gamma .
$$

Proof. (a) This is clear, it serves mainly to formulate (b).

(b) The case $\Gamma=\{\mathrm{id}\}$ is essentially [Lus6, Theorem 9.3]. The difference is that our conditions on $\lambda$ replace the conditions [Lus6, 9.1]. The general case follows easily under the assumption that $\Gamma$ fixes $u$.

Given $\mathfrak{t}^{\prime} \subset \mathfrak{t}$ we denote by $\operatorname{Mod}_{f, \mathfrak{t}^{\prime}}(\mathbb{H}(\tilde{\mathcal{R}}, k) \rtimes \Gamma)$ the category of finite dimensional $\mathbb{H}(\tilde{\mathcal{R}}, k) \rtimes \Gamma$-modules all whose $\mathcal{O}(\mathfrak{t})$-weights lie in $\mathfrak{t}^{\prime}$.

Corollary 2.1.5. Let $q$ be positive and let $u c \in T_{u n} T_{r s}$. The following categories are equivalent:

(a) $\operatorname{Mod}_{f, W_{0}^{\prime} u c}(\mathcal{H}(\mathcal{R}, q) \rtimes \Gamma)$ and $\operatorname{Mod}_{f,\left(W\left(R_{u}\right) \rtimes W_{F_{u}, u}^{\prime}\right) \log (c)}\left(\mathbb{H}\left(\tilde{\mathcal{R}}_{u}, k_{u}\right) \rtimes W_{F_{u}, u}^{\prime}\right)$,

(b) $\operatorname{Mod}_{f, W_{0}^{\prime} u T_{r s}}(\mathcal{H}(\mathcal{R}, q) \rtimes \Gamma)$ and $\operatorname{Mod}_{f, \mathfrak{a}}\left(\mathbb{H}\left(\tilde{\mathcal{R}}_{u}, k_{u}\right) \rtimes W_{F_{u}, u}^{\prime}\right)$.

These equivalences are compatible with parabolic induction.

Proof. (a) follows from Theorems 2.1.2 b and 2.1.4 b. Notice that the conditions (2.6) are automatically satisfied because $q$ is positive and $\log (c) \in \mathfrak{a}$, so $k_{u, \alpha} \in \mathbb{R}$ and $\langle\alpha, \log (c)\rangle \in \mathbb{R}$. If we sum that equivalence over all $W_{0}^{\prime} c \in T_{r s} / W_{0}^{\prime}$, we find (b). By [BaMo2, Theorem 6.2] or [Sol5, Proposition 5.3.a] these equivalences of categories are compatible with parabolic induction. 
2.2. The Langlands classification. In this section we discuss Langlands' classification of irreducible representations. Basically it reduces from general representations to tempered ones, and from there to the discrete series. Actually, Langlands proved this only in the setting of real reductive groups, but it holds just as well for $p$-adic reductive groups, affine Hecke algebras and graded Hecke algebras. We will only write down the results for affine Hecke algebras, the graded Hecke algebra case is completely analogous and can be found in [Eve, KrRa, Sol5.

An important tool to study $\mathcal{H}$-representations is restriction to the commutative subalgebra $\mathcal{A} \cong \mathcal{O}(T)$. We say that $t \in T$ is a weight of $(\pi, V)$ if there exists a $v \in V \backslash\{0\}$ such that $\pi(a) v=a(t) v$ for all $a \in \mathcal{A}$. It is easy to describe how the collection of $\mathcal{A}$-weights behaves under parabolic induction. Recall that

$$
W^{P}:=\left\{w \in W_{0}: w(P) \subset R_{0}^{+}\right\}
$$

is the set of minimal length representatives of $W_{0} / W\left(R_{P}\right)$.

Lemma 2.2.1. Let $\Gamma_{P}^{\prime}$ be a subgroup of $\Gamma_{P}$ and let $\sigma$ be a representation of $\mathcal{H}^{P} \rtimes \Gamma_{P}^{\prime}$. The $\mathcal{A}$-weights of $\operatorname{Ind}_{\mathcal{H}^{P} \rtimes \Gamma_{P}^{\prime}}^{\mathcal{H} \rtimes \Gamma}(\sigma)$ are the elements $\gamma w(t) \in T$, where $\gamma \in \Gamma, w \in W^{P}$ and $t$ is an $\mathcal{A}$-weight of $\sigma$.

Proof. From [BaMo1, Theorem 6.4] and the proof of Opd2, Proposition 4.20] we see that this holds in the case $\Gamma=\Gamma_{P}^{\prime}=\{\mathrm{id}\}$. For the general case we only have to observe that the operation $\pi \mapsto \pi \circ \psi_{\gamma}^{-1}$ on $\mathcal{H}$-representations has the effect $t \mapsto \gamma(t)$ on all $\mathcal{A}$-weights $t$.

Temperedness of a representation is defined via its $\mathcal{A}$-weights. Given $P \subseteq F_{0}$, we have the following positive cones in $\mathfrak{a}$ and in $T_{r s}$ :

$$
\begin{array}{llll}
\mathfrak{a}^{+} & =\left\{\mu \in \mathfrak{a}:\langle\alpha, \mu\rangle \geq 0 \forall \alpha \in F_{0}\right\}, & & T^{+}=\exp \left(\mathfrak{a}^{+}\right), \\
\mathfrak{a}_{P}^{+} & =\left\{\mu \in \mathfrak{a}_{P}:\langle\alpha, \mu\rangle \geq 0 \forall \alpha \in P\right\}, & & T_{P}^{+}=\exp \left(\mathfrak{a}_{P}^{+}\right), \\
\mathfrak{a}^{P+} & =\left\{\mu \in \mathfrak{a}^{P}:\langle\alpha, \mu\rangle \geq 0 \forall \alpha \in F_{0} \backslash P\right\}, & T^{P+} & =\exp \left(\mathfrak{a}^{P+}\right), \\
\mathfrak{a}^{P++} & =\left\{\mu \in \mathfrak{a}^{P}:\langle\alpha, \mu\rangle>0 \forall \alpha \in F_{0} \backslash P\right\}, & & T^{P++}=\exp \left(\mathfrak{a}^{P++}\right) .
\end{array}
$$

The anti-dual of $\mathfrak{a}^{*+}:=\left\{x \in \mathfrak{a}^{*}:\left\langle x, \alpha^{\vee}\right\rangle \geq 0 \forall \alpha \in F_{0}\right\}$ is

$$
\mathfrak{a}^{-}=\left\{\lambda \in \mathfrak{a}:\langle x, \lambda\rangle \leq 0 \forall x \in \mathfrak{a}^{*+}\right\}=\left\{\sum_{\alpha \in F_{0}} \lambda_{\alpha} \alpha^{\vee}: \lambda_{\alpha} \leq 0\right\} .
$$

Similarly, we define

$$
\mathfrak{a}_{P}^{-}=\left\{\sum_{\alpha \in P} \lambda_{\alpha} \alpha^{\vee} \in \mathfrak{a}_{P}: \lambda_{\alpha} \leq 0\right\} .
$$

The interior $\mathfrak{a}^{--}$of $\mathfrak{a}^{-}$equals $\left\{\sum_{\alpha \in F_{0}} \lambda_{\alpha} \alpha^{\vee}: \lambda_{\alpha}<0\right\}$ if $F_{0}$ spans $\mathfrak{a}^{*}$, and is empty otherwise. We write $T^{-}=\exp \left(\mathfrak{a}^{-}\right)$and $T^{--}=\exp \left(\mathfrak{a}^{--}\right)$. Let $t=|t| \cdot t|t|^{-1} \in$ $T_{r s} \times T_{u n}$ be the polar decomposition of $t$.

An $\mathcal{H}$-representation is called tempered if $|t| \in T^{-}$for all its $\mathcal{A}$-weights $t$, and anti-tempered if $|t|^{-1} \in T^{-}$for all such $t$. For infinite dimensional representations this is not entirely satisfactory, but we postpone a more detailed discussion to Section 3.2. Since all irreducible $\mathcal{H}$-representations have finite dimension, this vagueness does not cause any problems. Notice that our definition mimics HarishChandra's definition of admissible smooth tempered representations of reductive $p$-adic groups [Wal, Section III.2]. In that setting the crucial condition says that all exponents of such a representation must lie in certain cone. 
More restrictively we say that an irreducible $\mathcal{H}$-representation belongs to the discrete series (or simply: is discrete series) if $|t| \in T^{--}$, for all its $\mathcal{A}$-weights $t$. In particular, the discrete series is empty if $F_{0}$ does not span $\mathfrak{a}^{*}$. This is the analogue of Casselman's criterium for square integrable representations of semisimple $p$-adic groups [Cas, Theorem 4.4.6].

The notions tempered and discrete series apply equally well to $\mathcal{H} \rtimes \Gamma$, since that algebra contains $\mathcal{A}$ and the action of $\Gamma$ on $T$ preserves $T^{-}$. It follows more or less directly from the definitions that the correspondence of Theorem 2.1.4 preserves temperedness and provides a bijection between discrete series representations with the appropriate central characters, see [Slo2, (2.11)].

It easy to detect temperedness for $\mathcal{H}(\mathcal{R}, 1) \rtimes \Gamma=\mathbb{C}\left[W^{\prime}\right]=\mathbb{C}\left[X \rtimes W_{0}^{\prime}\right]$.

Lemma 2.2.2. A finite dimensional $\mathbb{C}\left[X \rtimes W_{0}^{\prime}\right]$-representation is tempered if and only if all its $\mathcal{A}$-weights lie in $T_{\text {un }}$.

This algebra has no discrete series representations, unless $X=0$.

Proof. Suppose that $V$ is a representation of this algebra, and that $t \in T$ is an $\mathcal{A}$-weight with weight space $V_{t}$. For every $g \in W_{0}^{\prime}, g V_{t}=V_{g(t)}$ is the $g(t)$-weight space of $V$, which shows that every element of the orbit $W_{0}^{\prime} t$ is an $\mathcal{A}$-weight of $V$. But $W_{0}^{\prime}|t|$ can only be contained in $T^{-}$if it equals the single element $1 \in T_{r s}$. Hence $V$ can only be tempered if $|t|=1$ for all its weights, or equivalently if all its weights lie in $T_{u n}$. By definition the latter condition also suffices for temperedness.

Unless $X=0$, the condition $|t|=1$ implies $|t| \notin T^{--}$, so $\mathbb{C}\left[X \rtimes W_{0}^{\prime}\right]$ has no discrete series representations.

The Langlands classification looks at parabolic subalgebras of $\mathcal{H}$ and irreducible representations of those that are essentially tempered. We will describe such representations with two data: a tempered representation and a "complementary" part of the central character. This is justified by the following result.

Lemma 2.2.3. Let $P \subset F_{0}, t_{P} \in T_{P}$ and $t^{P} \in T^{P}$.

(a) The map $\sigma \mapsto \sigma \circ \phi_{t^{P}}$ defines an equivalence between the categories of $\mathcal{H}_{P^{-}}$ representations with central character $W\left(R_{P}\right) t_{P} \in T_{P} / W\left(R_{P}\right)$ and of $\mathcal{H}^{P}$ representations with central character $W\left(R_{P}\right) t_{P} t^{P} \in T / W\left(R_{P}\right)$.

(b) Every irreducible $\mathcal{H}^{P}$-representation is of the form $\sigma \circ \phi_{t^{P}}$, where $\sigma$ is an irreducible $\mathcal{H}_{P}$-representation and $t^{P} \in T^{P}$. Both these data are unique modulo twists coming from $K_{P}=T^{P} \cap T_{P}$, as in (1.21).

Proof. (a) The kernel of $\phi_{t^{P}}$ followed by the quotient map $\mathcal{H}^{P} \rightarrow \mathcal{H}_{P}$ is generated (as an ideal) by $\left\{\theta_{x}-t^{P}(x): x \in X \cap\left(P^{\vee}\right)^{\perp}\right\}$. If $\rho$ is an $\mathcal{H}^{P}$-representation with central character $W\left(R_{P}\right) t_{P} t^{P}$, then the kernel of $\rho$ clearly contains these generators, so $\rho$ factors via $\phi_{t^{P}}$ and this quotient map.

(b) Let $\rho$ be an irreducible $\mathcal{H}^{P}$-representation with central character $W\left(R_{P}\right) t \in$ $T / W\left(R_{P}\right)$. Decompose $t=t_{P} t^{P} \in T_{P} T^{P}$. Then part (a) yields a unique irreducible $\mathcal{H}_{P}$-representation $\sigma$ such that $\rho=\sigma \circ \phi_{t^{P}}$. The only freedom in this constuction comes from elements $u \in K_{P}$. If we replace $t^{P}$ by $u t^{P}$, then part (a) again gives a unique $\sigma^{\prime}$ with $\rho=\sigma^{\prime} \circ \phi_{u t^{P}}$, and its follows directly that $\sigma^{\prime} \circ \psi_{u}=\sigma$.

A Langlands datum for $\mathcal{H}$ is a triple $(P, \sigma, t)$ such that

- $P \subseteq F_{0}$ and $\sigma$ is an irreducible tempered $\mathcal{H}_{P}$-representation;

- $t \in T^{P}$ and $|t| \in T^{P++}$. 
We say that two Langlands data $(P, \sigma, t)$ and $\left(P^{\prime}, \sigma^{\prime}, t^{\prime}\right)$ are equivalent if $P=P^{\prime}$ and the $\mathcal{H}^{P}$-representations $\sigma \circ \phi_{t}$ and $\sigma^{\prime} \circ \phi_{t^{\prime}}$ are equivalent.

Theorem 2.2.4 (Langlands classification).

(a) For every Langlands datum $(P, \sigma, t)$ the $\mathcal{H}$-representation $\pi(P, \sigma, t)=$ $\operatorname{Ind}_{\mathcal{H}^{P}}^{\mathcal{H}}\left(\sigma \circ \phi_{t}\right)$ has a unique irreducible quotient $L(P, \sigma, t)$.

(b) For every irreducible $\mathcal{H}$-representation $\pi$ there exists a Langlands datum $(P, \sigma, t)$, unique up to equivalence, such that $\pi \cong L(P, \sigma, t)$.

Proof. The author learned this result from a preliminary version of DeOp2, but unfortunately Delorme and Opdam did not include it in the final version. Yet the proof in the setting of affine Hecke algebras is much easier than for reductive groups. It is basically the same as the proof of Evens Eve for graded Hecke algebras; see also KrRa, Section 2.4]. In particular, part (b) can be proved as in these references, with an application of Lemma 2.2.3. For later use we rephrase the proof of (a) in our notation.

The dominance ordering on $\mathfrak{a}$ is defined by

$$
\lambda \leq \mu \text { if and only if }\langle\lambda, \alpha\rangle \leq\langle\mu, \alpha\rangle \text { for all } \alpha \in F_{0} .
$$

For $\alpha \in F_{0}$ we define $\delta_{\alpha} \in \mathfrak{a}_{F_{0}}$ by

$$
\left\langle\beta, \delta_{\alpha}\right\rangle=\left\{\begin{array}{lll}
1 & \text { if } & \alpha=\beta, \\
0 & \text { if } \quad \alpha \neq \beta \in F_{0} .
\end{array}\right.
$$

According to Langlands [Lan, Lemma 4.4], for every $\lambda \in \mathfrak{a}$ there is a unique subset $F(\lambda) \subset F_{0}$ such that $\lambda$ can be written as

$$
\lambda=\lambda^{F_{0}}+\sum_{\alpha \in F_{0} \backslash F(\lambda)} c_{\alpha} \delta_{\alpha}+\sum_{\alpha \in F(\lambda)} d_{\alpha} \alpha^{\vee} \text { with } \lambda^{F_{0}} \in \mathfrak{a}^{F_{0}}, c_{\alpha}>0, d_{\alpha} \leq 0 .
$$

We put $\lambda_{0}=\sum_{\alpha \in F_{0} \backslash F(\lambda)} c_{\alpha} \delta_{\alpha} \in \mathfrak{a}^{+}$. According to [KrRa, (2.13)],

$$
(w \mu)_{0}<\mu_{0} \text { for all } \mu \in \mathfrak{a}_{P}^{-} \oplus \mathfrak{a}^{P++}, w \in W^{P} \backslash\{1\} .
$$

By the definition of a Langlands datum $\log |s| \in \mathfrak{a}_{P}^{-} \oplus \mathfrak{a}^{P++}$ for every $\mathcal{A}$-weight $s$ of $\sigma \circ \phi_{t}$. Choose $s$ such that $(\log |s|)_{0}$ is maximal with respect to the dominance order. By Lemma 2.2.1 and (2.13) $(\log |s|)_{0}$ is also maximal for $s$ regarded as an $\mathcal{A}$-weight of $\pi(P, \sigma, t)$.

Suppose that $\rho$ is an $\mathcal{H}$-submodule of $\pi(P, \sigma, t)$ of which $s$ is an $\mathcal{A}$-weight. By the maximality of $s, \rho$ must contain the $s$-weight space of $\sigma \circ \phi_{t}$. The irreduciblity of $\sigma$ implies that $\rho$ contains the $\mathcal{H}^{P}$-submodule $1 \otimes_{\mathcal{H}^{P}} V_{\sigma} \subset \operatorname{Ind}_{\mathcal{H}^{P}}^{\mathcal{H}}\left(\sigma \circ \phi_{t}\right)$, and therefore $\rho=\pi(P, \sigma, t)$. Thus the sum of all proper submodules is again proper, which means that $\pi(P, \sigma, t)$ has a unique maximal submodule and a unique irreducible quotient.

Theorem 2.2 .4 can be regarded as the analogue of the Langlands classification for connected reductive $p$-adic groups. For disconnected reductive groups the classification is no longer valid as such, it has to be modified. In the case that the component group is abelian, this is worked out in [BaJa1, via reduction to cyclic component groups.

We work with a diagram automorphism group $\Gamma$ which is more general than a component group and does not have to be abelian. For use in Section 2.3 we have to extend Theorem 2.2 .4 to this setting. 
There is a natural action of $\Gamma$ on Langlands data, by

$$
\gamma(P, \sigma, t)=\left(\gamma(P), \sigma \circ \psi_{\gamma}^{-1}, \gamma(t)\right) .
$$

Every Langlands datum yields a packet of irreducible quotients, and all data in one $\Gamma$-orbit lead to the same packet. For $\gamma \in \Gamma_{P}$ the Langlands classification for $\mathcal{H}^{P}$ shows that the irreducible $\mathcal{H}^{P}$-representations $\sigma \circ \psi_{\gamma} \circ \phi_{\gamma(t)}$ and $\sigma \circ \phi_{t}$ are equivalent if and only if $\gamma(P, \sigma, t)=(P, \sigma, t)$.

To get a more precise statement one needs Clifford theory, as for example in RaRa or CuRe, Section 53]. Let $\Gamma_{P, \sigma, t}$ be the isotropy group of the Langlands datum $(P, \sigma, t)$. In Sol5, Appendix A] a 2-cocycle $\kappa$ of $\Gamma_{P, \sigma, t}$ is constructed, giving rise to a twisted group algebra $\mathbb{C}\left[\Gamma_{P, \sigma, t}, \kappa\right]$. We define a Langlands datum for $\mathcal{H} \rtimes \Gamma$ as a quadruple $(P, \sigma, t, \rho)$, where

- $(P, \sigma, t)$ is a Langlands datum for $\mathcal{H}$;

- $\rho$ is an irreducible representation of $\mathbb{C}\left[\Gamma_{P, \sigma, t}, \kappa\right]$.

The action (2.14) extends naturally to Langlands data for $\mathcal{H} \rtimes \Gamma$, since $\psi_{\gamma}^{-1}$ induces an isomorphism between the relevant twisted group algebras.

From such a Langlands datum we can construct the $\mathcal{H}_{P} \rtimes \Gamma_{P, \sigma, t}$-representation $\sigma \otimes \rho$ and the $\mathcal{H} \rtimes \Gamma$-representation

$$
\pi^{\Gamma}(P, \sigma, t, \rho):=\operatorname{Ind}_{\mathcal{H}^{P} \rtimes \Gamma_{P, \sigma, t}}^{\mathcal{H} \rtimes \Gamma}\left(\left(\sigma \circ \phi_{t}\right) \otimes \rho\right)=\operatorname{Ind}_{\mathcal{H}^{P} \rtimes \Gamma_{P, \sigma, t}}^{\mathcal{H} \rtimes \Gamma}\left((\sigma \otimes \rho) \circ \phi_{t}\right) .
$$

If $Q \supset P$, then $(P, \sigma, t, \rho)$ can also be considered as a Langlands datum for $\mathcal{H}^{Q} \rtimes \Gamma_{Q}$, and we denote the corresponding $\mathcal{H}^{Q} \rtimes \Gamma_{Q}$-representation by $\pi^{Q, \Gamma_{Q}}(P, \sigma, t, \rho)$. In particular, $\pi^{P, \Gamma_{P}}(P, \sigma, t, \rho)$ is an irreducible $\mathcal{H}^{P} \rtimes \Gamma_{P}$-representation.

Corollary 2.2.5 (extended Langlands classification).

(a) The $\mathcal{H} \rtimes \Gamma$-representation $\pi^{\Gamma}(P, \sigma, t, \rho)$ has a unique irreducible quotient $L^{\Gamma}(P, \sigma, t, \rho)$.

(b) For every irreducible $\mathcal{H} \rtimes \Gamma$-representation $\pi$ there exists a Langlands datum $(P, \sigma, t, \rho)$, unique modulo the action of $\Gamma$, such that $\pi \cong L^{\Gamma}(P, \sigma, t, \rho)$.

(c) $L^{\Gamma}(P, \sigma, t, \rho)$ and $\pi^{\Gamma}(P, \sigma, t, \rho)$ are tempered if and only if $P=F_{0}$ and $t \in$ $T_{u n}^{F_{0}}$.

Proof. (a) and (b) By [Sol5, Theorem A.1] the $\mathcal{H} \rtimes \Gamma$-representation

$$
\operatorname{Ind}_{\mathcal{H} \rtimes \Gamma_{P, \sigma, t}}^{\mathcal{H} \rtimes \Gamma}(L(P, \sigma, t) \otimes \rho)
$$

is irreducible, and every irreducible $\mathcal{H} \rtimes \Gamma$-representation is of this form, for a Langlands datum which is unique modulo $\Gamma$. By construction (2.16) is a quotient of $\pi(P, \sigma, t, \rho)$. It is the unique irreducible quotient by Theorem 2.2.4 a and because $\rho$ is irreducible.

(c) If $P \subsetneq F_{0}$, then $L(P, \sigma, t, \rho)$ and $\pi^{\Gamma}(P, \sigma, t, \rho)$ are never tempered. Indeed, $|t| \notin T^{-}$, so $|r t| \notin T^{-}$for any $\mathcal{A}_{P}$-weight $r$ of $\sigma$. But the construction of $L(P, \sigma, t)$ in the proof of 2.2.4 a is precisely such that the $\mathcal{A}$-weight $r t$ of $\pi(P, \sigma, t)$ survives to the Langlands quotient. Since the group $\Gamma$ preserves $T^{-}$, its presence does not affect temperedness.

Now assume that $P=F_{0}$. Since $T^{F^{0}++} \subset T_{r s}^{F_{0}}$ and $T^{-} \cap T_{r s}^{F_{0}}=\{1\}$, this representation can only be tempered if $|t|=1$. In that case $\sigma$ and $\pi^{\Gamma}(P, \sigma, t, \rho)$ have the same absolute values of $\mathcal{A}$-weights, modulo $\Gamma$. But $\Gamma T^{-}=T^{-}$, so the temperedness of $\pi^{\Gamma}(P, \sigma, t, \rho)$ and $L^{\Gamma}(P, \sigma, t, \rho)$ follows from that of $\sigma$. 
For connected reductive $p$-adic groups the Langlands quotient always appears with multiplicity one in the standard representation of which it is a quotient. Although not stated explicitly in most sources, that is already part of the proof, see Kon or [Sol4, Theorem 2.15]. This also holds for reductive $p$-adic groups with a cyclic component group BaJa2.

Closer examination of the proof of Theorem 2.2.4 allows us to generalize and improve upon this in our setting. Let $W\left(R_{P}\right) r_{\sigma} \in T_{P} / W\left(R_{P}\right)$ be the central character of $\sigma$. Then $\left|r_{\sigma}\right| \in T_{P, r s}=\exp \left(\mathfrak{a}_{P}\right)$, so we can define

$$
c c_{P}(\sigma):=W\left(R_{P}\right) \log \left|r_{\sigma}\right| \in \mathfrak{a}_{P} / W\left(R_{P}\right) .
$$

Since the inner product on $\mathfrak{a}$ is $W_{0}^{\prime}$-invariant, the number $\left\|c c_{P}(\sigma)\right\|$ is well defined.

Lemma 2.2.6. Let $(P, \sigma, t, \rho)$ and $\left(P, \sigma^{\prime}, t, \rho^{\prime}\right)$ be Langlands data for $\mathcal{H} \rtimes \Gamma$.

(a) The functor $\operatorname{Ind}_{\mathcal{H}^{P} \rtimes \Gamma_{P}}^{\mathcal{H} \rtimes \Gamma_{P}}$ induces an isomorphism

$$
\begin{aligned}
& \operatorname{Hom}_{\mathcal{H}^{P} \rtimes \Gamma_{P}}\left(\pi^{P, \Gamma_{P}}(P, \sigma, t, \rho), \pi^{P, \Gamma_{P}}\left(P, \sigma^{\prime}, t, \rho^{\prime}\right)\right) \\
& \cong \operatorname{Hom}_{\mathcal{H} \rtimes \Gamma}\left(\pi^{\Gamma}(P, \sigma, t, \rho), \pi^{\Gamma}\left(P, \sigma^{\prime}, t, \rho^{\prime}\right)\right) .
\end{aligned}
$$

These spaces are one-dimensional if $(\sigma, t, \rho)$ and $\left(\sigma^{\prime}, t, \rho^{\prime}\right)$ are $\Gamma_{P}$-conjugate, and zero otherwise.

(b) Suppose that $L^{\Gamma}(Q, \tau, s, \nu)$ is a constituent of $\pi^{\Gamma}(P, \sigma, t, \rho)$, but not $L^{\Gamma}(P, \sigma, t, \rho)$. Then $P \subset Q$ and $\left\|c c_{P}(\sigma)\right\|<\left\|c c_{Q}(\tau)\right\|$.

Proof. (a) We use the notation from (2.12). For any weight $s$ of $\sigma \circ \phi_{t}$ we have $\log \left|s t^{-1}\right| \in \mathfrak{a}_{P}^{-}$and $(\log |s|)_{0}=(\log |t|)_{F_{0}}$, where the subscript $F_{0}$ refers to the decomposition of elements of $\mathfrak{t}$ with respect to $\mathfrak{t}=\mathfrak{t}_{F_{0}} \oplus \mathfrak{t}^{F_{0}}$. Let $s^{\prime}$ be a weight of $\sigma^{\prime} \circ \phi_{t}$. By (2.13)

$$
\left(w \log \left|s^{\prime}\right|\right)_{0}<\left(\log \left|s^{\prime}\right|\right)_{0}=(\log |t|)_{F_{0}} \quad \forall w \in W^{P} \backslash\{\text { id }\},
$$

with respect to the dominance order on $\mathfrak{a}_{F_{0}}^{*}$. Since $(\gamma \lambda)_{0}=\gamma\left(\lambda_{0}\right)$ for all $\lambda \in \mathfrak{a}$ and $\gamma \in \Gamma$, we get

$$
\left\|\left(\gamma w \log \left|s^{\prime}\right|\right)_{0}\right\|<\left\|(\log |t|)_{F_{0}}\right\| \quad \forall \gamma \in \Gamma, w \in W^{P} \backslash\{\operatorname{id}\} .
$$

In particular, $\gamma w\left(s^{\prime}\right)$ with $w \in W^{P}$ can only equal the weight $s$ of $\sigma \circ \phi_{t}$ if $w=1$. Let $v_{s} \in V_{\sigma \otimes \rho}$ be a nonzero weight vector. Since $\pi^{P, \Gamma_{P}}(P, \sigma, t, \rho)$ is an irreducible $\mathcal{H}^{P} \rtimes \Gamma_{P^{-}}$-representation, $1 \otimes v_{s} \in(\mathcal{H} \rtimes \Gamma) \otimes_{\mathcal{H}^{P} \rtimes \Gamma_{P, \sigma, t}} V_{\sigma \otimes \rho}$ is cyclic for $\pi^{\Gamma}(P, \sigma, t, \rho)$. Therefore the map

$$
\operatorname{Hom}_{\mathcal{H} \rtimes \Gamma}\left(\pi^{\Gamma}(P, \sigma, t, \rho), \pi^{\Gamma}\left(P, \sigma^{\prime}, t, \rho^{\prime}\right)\right) \rightarrow \pi^{\Gamma}\left(P, \sigma^{\prime}, t, \rho^{\prime}\right): f \mapsto f\left(1 \otimes v_{s}\right)
$$

is injective. By (2.19) the $s$-weight space of $\pi^{\Gamma}\left(P, \sigma^{\prime}, t, \rho^{\prime}\right)$ is contained in $1 \otimes V_{\sigma^{\prime}} \otimes \rho^{\prime}$. So $f\left(1 \otimes v_{s}\right) \in 1 \otimes V_{\sigma^{\prime} \otimes \rho^{\prime}}$ and multiplying by $\mathcal{H}^{P} \rtimes \Gamma_{P}$ yields

$$
f\left(\mathbb{C}\left[\Gamma_{P}\right] \otimes_{\Gamma_{P, \sigma, t}} V_{\sigma \otimes \rho}\right) \subset \mathbb{C}\left[\Gamma_{P}\right] \otimes_{\Gamma_{P, \sigma^{\prime}, t}} V_{\sigma^{\prime} \otimes \rho^{\prime}} .
$$

Thus any $f \in \operatorname{Hom}_{\mathcal{H} \rtimes \Gamma}\left(\pi^{\Gamma}(P, \sigma, t, \rho), \pi^{\Gamma}\left(P, \sigma^{\prime}, t, \rho^{\prime}\right)\right)$ lies in

$$
\operatorname{Ind}_{\mathcal{H}^{P} \rtimes \Gamma_{P}}^{\mathcal{H} \rtimes \Gamma_{P}}\left(\operatorname{Hom}_{\mathcal{H}^{P} \rtimes \Gamma_{P}}\left(\pi^{P, \Gamma_{P}}(P, \sigma, t, \rho), \pi^{P, \Gamma_{P}}\left(P, \sigma^{\prime}, t, \rho^{\prime}\right)\right)\right) .
$$

From (2.20) we see that this induction functor is injective on homomorphisms. The modules in (2.21) are irreducible, so the dimension of (2.21) is zero or one. By Corollary 2.2.5 b it is nonzero if and only if $(\sigma, t, \rho)$ and $\left(\sigma^{\prime}, t, \rho^{\prime}\right)$ are $\Gamma_{P}$-conjugate.

(b) The proofs of Theorem 2.2.4 a and Corollary 2.2.5 a show that $L^{\Gamma}(P, \sigma, t, \rho)$ is the unique irreducible subquotient of $\pi^{\Gamma}(P, \sigma, t, \rho)$ which has an $\mathcal{A}$-weight $t_{L}$ 
with $\left(\log \left|t_{L}\right|\right)_{0}=(\log |t|)_{F_{0}}$. Moreover, all $\mathcal{A}$-weights $s^{\prime}$ of proper submodules of $\pi^{\Gamma}(P, \sigma, t, \rho)$ satisfy $\left(\log \left|s^{\prime}\right|\right)_{0}<(\log |t|)_{F_{0}}$, with the notation of (2.12). In particular, for the subquotient $L^{\Gamma}(Q, \tau, s, \nu)$ of $\pi^{\Gamma}(P, \sigma, t, \rho)$ we find that

$$
(\log |s|)_{F_{0}}=\left(\log \left|s^{\prime}\right|\right)_{0}<(\log |t|)_{F_{0}} .
$$

Since $\log |s| \in \mathfrak{a}^{Q++}$ and $\log |t| \in \mathfrak{a}^{P++}$, this implies $P \subset Q$ and

$$
\left\|(\log |s|)_{F_{0}}\right\|<\left\|(\log |t|)_{F_{0}}\right\| \text {. }
$$

According to Lemma 2.2 .1 all constituents of $\pi^{\Gamma}(P, \sigma, t, \rho)$ have central character $W_{0}^{\prime}\left(r_{\sigma} t\right) \in T / W_{0}^{\prime}$. The same goes for $(Q, \tau, s, \nu)$, so $r_{\sigma} t$ and $r_{\tau} s$ lie in the same $W_{0} \rtimes \Gamma$-orbit and

$$
W_{0}^{\prime}\left(\log \left|r_{\sigma} t\right|\right)_{F_{0}}=W_{0}^{\prime}\left(\log \left|r_{\tau} s\right|\right)_{F_{0}} .
$$

By definition $(\log |t|)_{F_{0}} \perp \mathfrak{t}_{P}$ and $(\log |s|)_{F_{0}} \perp \mathfrak{t}_{Q}$, so

$$
\begin{aligned}
& \left\|\Re\left(c c_{P}(\sigma)\right)\right\|^{2}+\left\|(\log |t|)_{F_{0}}\right\|^{2}=\left\|\left(\log \left|r_{\sigma} t\right|\right)_{F_{0}}\right\|^{2} \\
& \quad=\left\|\left(\log \left|r_{\tau} s\right|\right)_{F_{0}}\right\|^{2}=\left\|\Re\left(c c_{Q}(\tau)\right)\right\|^{2}+\left\|(\log |s|)_{F_{0}}\right\|^{2} .
\end{aligned}
$$

Finally we use (2.22).

2.3. From $\mathcal{H}$-representations to $W$-representations. Given an algebra or group $A$, let $\operatorname{Irr}(A)$ be the collection of (equivalence classes of) irreducible complex $A$-representations. Let $G_{\mathbb{Z}}(A)=G_{\mathbb{Z}}\left(\operatorname{Mod}_{f}(A)\right)$ denote the Grothendieck group of the category of finite length complex representations of $A$, and write $G_{\mathbb{F}}(A)=G_{\mathbb{Z}}(A) \otimes_{\mathbb{Z}} \mathbb{F}$ for any field $\mathbb{F}$.

The classical Springer correspondence $[\mathrm{Spr}]$ realizes all irreducible representation of a finite reflection group $W_{0}$ in the top cohomology of the associated flag variety. Kazhdan-Lusztig theory (see KaLu2, [Xi]) allows one to interpret this as a bijection between $\operatorname{Irr}\left(W_{0}\right)$ and a certain collection of irreducible representations of an affine Hecke algebra with equal parameters. As such, the finite Springer correspondence is a specialisation of an affine Springer correspondence between $\operatorname{Irr}(W(\mathcal{R}))$ and $\operatorname{Irr}(\mathcal{H}(\mathcal{R}, q))$, see [Lus5, Section 8]. The proof of Kazhdan and Lusztig requires that $\mathcal{R}$ is of simply connected type and that $q$ is an equal parameter function whose value is either 1 or not a root of unity. Reeder [Ree2, Theorem 3.5.4] showed that the resulting parametrization of irreducible $\mathcal{H}(\mathcal{R}, q)$-modules remains valid without simple connectedness. We will prove an analogue of this result for all extended affine Hecke algebras with unequal (but positive) parameters.

For any $\mathbb{H} \rtimes \Gamma$-representation $\pi$, let $\left.\pi\right|_{W_{0} \rtimes \Gamma}=\left.\pi\right|_{W_{0}^{\prime}}$ be the restriction of $\pi$ to the subalgebra $\mathbb{C}\left[W_{0}^{\prime}\right]=\mathbb{C}\left[W_{0} \rtimes \Gamma\right] \subset \mathbb{H} \rtimes \Gamma$. Let $\operatorname{Irr}_{0}(\mathbb{H} \rtimes \Gamma)$ be the collection of (equivalence classes of) irreducible tempered $\mathbb{H} \rtimes \Gamma$-representations with real central character. In [Sol6, Theorem 6.5.c] the author proved that the set

$$
\left\{\left.\pi\right|_{W_{0} \rtimes \Gamma}: \pi \in \operatorname{Irr}_{0}(\mathbb{H} \rtimes \Gamma)\right\}
$$

is a $\mathbb{Q}$-basis of $G_{\mathbb{Q}}\left(W_{0} \rtimes \Gamma\right)$. When $\Gamma$ is trivial, this is a kind of Springer correspondence for finite Weyl groups. The only problem is that $\left.\pi\right|_{W_{0}}$ may be reducible, but that could be solved by picking a suitable (a priori not canonical) irreducible subrepresentation of $\left.\pi\right|_{W_{0}}$.

In fact it is known from [Ciu, Corollary 3.6] that in many cases the matrix that expresses (2.24) in terms of irreducible $W_{0} \rtimes \Gamma$-representations is unipotent and upper triangular (with respect to a suitable ordering). That would provide a natural 
Springer correspondence for graded Hecke algebras with arbitrary parameters, but unfortunately that improvement is still open in our generality.

Theorem 2.3.1. There exists a unique system of maps

$$
\zeta^{*}: \operatorname{Irr}(\mathcal{H} \rtimes \Gamma) \rightarrow \operatorname{Mod}_{f}\left(X \rtimes W_{0}^{\prime}\right),
$$

for all extended affine Hecke algebras $\mathcal{H} \rtimes \Gamma$ with positive parameters, such that:

(a) The image of $\zeta^{*}$ is a $\mathbb{Q}$-basis of $G_{\mathbb{Q}}\left(X \rtimes W_{0}^{\prime}\right)$.

(b) $\zeta^{*}$ preserves the unitary part of the central character.

(c) $\zeta^{*}(\pi)$ is tempered if and only if $\pi$ is tempered.

(d) Let $u \in T_{u n}$, let $\tilde{\pi} \in \operatorname{Irr}_{0}\left(\mathbb{H}\left(\tilde{\mathcal{R}}_{u}, k_{u}\right) \rtimes W_{F_{u}, u}^{\prime}\right)$ and let $\tilde{\pi} \circ \Phi_{u}$ be the $\mathcal{H}\left(\mathcal{R}_{u}, q_{u}\right)$ $\rtimes W_{F_{u}, u}^{\prime}$-representation associated to it via Theorem 2.1.4(b). Then

$$
\zeta^{*}\left(\operatorname{Ind}_{\mathcal{H}\left(\mathcal{R}_{u}, q_{u}\right) \rtimes W_{F_{u}, u}^{\prime}}^{\mathcal{H} \rtimes}\left(\tilde{\pi} \circ \Phi_{u}\right)\right)=\operatorname{Ind}_{X \rtimes W_{u}^{\prime}}^{X \rtimes W_{0}^{\prime}}\left(\left.\mathbb{C}_{u} \otimes \tilde{\pi}\right|_{W_{u}^{\prime}}\right),
$$

where $\mathbb{C}_{u}$ denotes the one-dimensional $X$-representation with character $u$.

(e) If $(P, \sigma, t, \rho)$ is a Langlands datum for $\mathcal{H} \rtimes \Gamma$, then

$$
\zeta^{*}\left(L^{\Gamma}(P, \sigma, t, \rho)\right)=\operatorname{Ind}_{X \rtimes\left(W\left(R_{P}\right) \rtimes \Gamma_{P, \sigma, t}\right)}^{X \rtimes W_{0}^{\prime}}\left(\zeta^{*}(\sigma \otimes \rho) \circ \phi_{t}\right) .
$$

Proof. In view of Corollary 2.1.5, properties (b) and (d) determine $\zeta^{*}$ uniquely for all irreducible tempered representations. A glance at Lemma 2.2.2 shows that $\zeta^{*}$ preserves temperedness.

Next, Corollary 2.2.5.b and property (e) determine $\zeta^{*}$ for all irreducible representations. By Corollary 2.2.5 every nontempered irreducible $\mathcal{H} \rtimes \Gamma$-representation $\pi$ is of the form $L(P, \sigma, t, \rho)$ for some Langlands datum with $t \notin T_{u n}$. By construction all $\mathcal{A}$-weights of $\zeta^{*}(\sigma \otimes \rho)$ lie in $T_{u n}$, so by property (e) the absolute values of $\mathcal{A}$-weights of $\zeta^{*}(\pi)$ lie in $W_{0}^{\prime}|t|$. Together with Lemma 2.2 .2 this shows that $\zeta^{*}(\pi)$ is not tempered.

Now we have (b)-(e), let us turn to (a). By Corollary 2.1.5 and the result mentioned in (2.24), (a) holds if we restrict to tempered representations on both sides. The proof that this restriction is unnecessary is more difficult, we postpone it to Section 3.4,

We will see in Corollary 4.4 .3 that on tempered representations $\zeta^{*}$ is given by composition with an algebra homomorphism between suitable completions of $\mathbb{C}[X \rtimes$ $\left.\left(W_{0} \rtimes \Gamma\right)\right]$ and $\mathcal{H} \rtimes \Gamma$. That is not possible for all irreducible representations, since sometimes $\zeta^{*}$ does not preserve the dimensions of representations. More precisely, $\zeta^{*}$ preserves the dimension of an irreducible tempered representation, since property (d) does so. By contrast, the right-hand side of property (e) has the same dimension as $\pi(P, \sigma, t, \rho)$. Thus $\zeta^{*}$ preserves the dimension of $L(P, \sigma, t, \rho)$ if this Langlands quotient equals $\pi(P, \sigma, t, \rho)$, and increases the dimension otherwise.

Since the principal series representations $M(t)=\operatorname{Ind}_{\mathcal{A}}^{\mathcal{H} \rtimes \Gamma} \mathbb{C}_{t}$ and $\operatorname{Ind}_{X}^{X \rtimes W_{0}^{\prime}} \mathbb{C}_{t}$ are irreducible for all $t$ in a Zariski-open dense subset of $T, \zeta^{*}$ preserves irreducibility on a large part of $\operatorname{Irr}(\mathcal{H} \rtimes \Gamma)$. To make a nice affine Springer correspondence out of $\zeta^{*}$, one would have to modify it so that it always respects irreducibility. This would boil down to refining (2.24) (preferably in a canonical way) to map that preserves irreducibility.

For some applications the following variation on $\zeta^{*}$ is more convenient: 
Corollary 2.3.2. Theorem 2.3.1 also holds with condition (e) replaced by

$$
\left(e^{\vee}\right) \quad \zeta^{\vee}\left(\pi^{\Gamma}(P, \sigma, t, \rho)\right)=\operatorname{Ind}_{X \rtimes\left(W\left(R_{P}\right) \rtimes \Gamma_{P, \sigma, t}\right)}^{X \rtimes W_{0}^{\prime}}\left(\zeta^{\vee}(\sigma \otimes \rho) \circ \phi_{t}\right) .
$$

The resulting map

$$
\zeta^{\vee}: G_{\mathbb{Z}}(\mathcal{H} \rtimes \Gamma) \rightarrow G_{\mathbb{Z}}\left(X \rtimes W_{0}^{\prime}\right)
$$

commutes with parabolic induction.

Proof. This follows from Theorem 2.3.1 and Lemma 2.2.6. $\mathrm{b}$.

The disadvantage of $\zeta^{\vee}$ compared to $\zeta^{*}$ is that it sends some irreducible $\mathcal{H} \rtimes$ $\Gamma$-representations to virtual $W \rtimes \Gamma$-representations. Suppose for example that a principal series representation $M(t)$ with $t \in T^{++}$has only one quotient $\pi$ and only one subrepresentation $\delta$, which is tempered. (This occurs already for $\mathcal{R}$ of type $A_{1}^{(1)}$.) Then

$$
\zeta^{\vee}(\pi)=\zeta^{\vee}(M(t))-\zeta^{\vee}(\delta)=\operatorname{Ind}_{X}^{X \rtimes W_{0}^{\prime}} \mathbb{C}_{t}-\zeta^{\vee}(\delta) \in G_{\mathbb{Z}}\left(X \rtimes W_{0}^{\prime}\right),
$$

and the right-hand side is no ordinary representation because $\zeta^{\vee}(\delta)$ is tempered and $\operatorname{Ind}_{X}^{X \rtimes W_{0}^{\prime}} \mathbb{C}_{t}$ has no $X$-weights in $T_{u n}$.

Of course there also exist versions of Theorem 2.3.1 and Corollary 2.3.2 for graded Hecke algebras. They can easily be deduced from the above using Theorem 2.1.4.b.

\section{Parabolically induced Representations}

Parabolic induction is a standard tool to create a large supply of interesting representations of reductive groups, Hecke algebras and related objects. In line with Harish-Chandra's philosophy of the cusp form, every irreducible tempered representation of an affine Hecke algebra can be obtained via unitary induction of a discrete series representation of a parabolic subalgebra. With the Langlands classification we can also reach irreducible representations that are not tempered.

Hence we consider induction data $\xi=(P, \delta, t)$, where $\delta$ is a discrete series representation of $\mathcal{H}_{P}$ and $t \in T^{P}$ is an induction parameter. With this we associate a representation $\pi(\xi)=\operatorname{Ind}_{\mathcal{H}^{P}}^{\mathcal{H}}\left(\delta \circ \phi_{t}\right)$. Among these are the principal series representations, which already exhaust the dual space of $\mathcal{H}$. But that is not very satisfactory, since a principal series representation can have many irreducible subquotients, and it is not so easy to determine them; see Ree1.

Instead we are mostly interested in induction data $\xi$ for which $|t|$ is positive (in an appropriate sense) and in irreducible quotients of $\pi(\xi)$, because the Langlands classification applies to these. In Theorem 3.3 .2 we construct, for every irreducible $\mathcal{H}$-representation $\rho$, an essentially unique induction datum $\xi^{+}(\rho)$, such that $\rho$ is a quotient of $\pi\left(\xi^{+}(\rho)\right)$. However, in general $\pi\left(\xi^{+}(\rho)\right)$ has more than one irreducible quotient.

Another important theme in this section are intertwining operators between induced representations of the form $\pi(\xi)$. Their definition and most important properties stem from the work of Opdam and Delorme Opd2, DeOp1. Like in the setting of reductive groups, it is already nontrivial to show that normalized intertwining operators are regular on unitary induced representations. Under favorable circumstances such intertwining operators span $\operatorname{Hom}_{\mathcal{H}}\left(\pi(\xi), \pi\left(\xi^{\prime}\right)\right)$. This was already known DeOp1] for unitary induction data $\xi, \xi^{\prime}$, in which case $\pi(\xi)$ and $\pi\left(\xi^{\prime}\right)$ are tempered representations. We generalize this to pairs of positive induction data 
(Theorem 3.3.1). Crucial in all these considerations is the Schwartz algebra $\mathcal{S}$ of $\mathcal{H}$, the analogue of the Harish-Chandra-Schwartz algebra of a reductive $p$-adic group.

For the geometry of the dual space of $\mathcal{H}$ it is important to understand the number $n(\xi)$ of irreducible $\mathcal{H}$-representations $\rho$ with $\xi^{+}(\rho)$ equivalent to $\xi$. This is governed by a groupoid $\mathcal{G}$ that keeps track of all intertwining operators. Indeed, if $t \mapsto \xi_{t}$ is a continuous path of induction data such that all $\xi_{t}$ have the same isotropy group in $\mathcal{G}$, then $n\left(\xi_{t}\right)$ is constant along this path (Proposition 3.4.1).

From this we deduce that the dual of $\mathcal{H}$ is a kind of complexification of the tempered dual of $\mathcal{H}$. As a topological space, the tempered dual is built from certain algebraic subvarieties of compact tori, each with a multiplicity. In this picture the dual of $\mathcal{H}$ is built from the corresponding complex subvarieties of complex algebraic tori, with the same multiplicities. This geometric description is used to finish the proof of Theorem 2.3.1.

3.1. Unitary representations and intertwining operators. As for Lie groups, the classification of the unitary dual of an affine Hecke algebra appears to be considerably more difficult than the classification of the full dual or of the tempered dual. This is an open problem that we will not discuss in this paper; cf. BaMo2, $\mathrm{BaCi}$. Nevertheless, we will use unitarity arguments, mainly to show that certain representations are completely reducible. The algebra $\mathcal{H} \rtimes \Gamma$ is endowed with a sesquilinear involution $*$ and a trace $\tau$, defined by

$$
\begin{aligned}
& \left(z N_{w} \gamma\right)^{*}=\bar{z} \gamma^{-1} N_{w^{-1}} \quad z \in \mathbb{C}, w \in W, \gamma \in \Gamma, \\
& \tau\left(z N_{w} \gamma\right)= \begin{cases}z & \text { if } \gamma=w=e, \\
0 & \text { otherwise. }\end{cases}
\end{aligned}
$$

Since $q$ is real-valued, this $*$ is anti-multiplicative and $\tau$ is positive. These give rise to an Hermitian inner product on $\mathcal{H} \rtimes \Gamma$ :

$$
\left\langle h, h^{\prime}\right\rangle_{\tau}=\tau\left(h^{*} h^{\prime}\right) \quad h, h^{\prime} \in \mathcal{H} \rtimes \Gamma .
$$

A short calculation using the multiplication rules (1.4) shows that the basis $\left\{N_{w} \gamma\right.$ : $w \in W, \gamma \in \Gamma\}$ of $\mathcal{H} \rtimes \Gamma$ is orthonormal for this inner product.

We note that $\Gamma$ acts on $\mathcal{H}$ by ${ }^{*}$-automorphisms, and that $\mathcal{H}, \mathcal{H}\left(W_{0}, q\right)$ and $\mathbb{C}[\Gamma]$ are ${ }^{*}$-subalgebras of $\mathcal{H} \rtimes \Gamma$. In general $\mathcal{A}$ is not a ${ }^{*}$-subalgebra of $\mathcal{H}$. For $x \in X$ Opd2, Proposition 1.12] tells us that

$$
\theta_{x}^{*}=N_{w_{0}} \theta_{-w_{0}(x)} N_{w_{0}}^{-1},
$$

where $w_{0}$ is the longest element of the Coxeter group $W_{0}$.

Let $\Gamma_{P}^{\prime}$ be a subgroup of $\Gamma_{P}$ and let $\tau$ be a $\mathcal{H}^{P} \rtimes \Gamma_{P}^{\prime}$-representation on an inner product space $V_{\tau}$. By default we will endow the vector space

$$
\mathcal{H} \rtimes \Gamma \otimes_{\mathcal{H}^{P} \rtimes \Gamma_{P}^{\prime}} V_{\tau} \cong \mathbb{C}\left[\Gamma W^{P}\right] \otimes_{\mathbb{C}\left[\Gamma_{P}^{\prime}\right]} V_{\tau}
$$

with the inner product

$$
\left\langle h \otimes v, h^{\prime} \otimes v^{\prime}\right\rangle=\tau\left(h^{*} h^{\prime}\right)\left\langle v, v^{\prime}\right\rangle \quad h, h^{\prime} \in \mathbb{C}\left[\Gamma W^{P}\right], v, v^{\prime} \in V_{\tau} .
$$

Recall that a representation $\pi$ of $\mathcal{H} \rtimes \Gamma$ on a Hilbert space is unitary if $\pi\left(h^{*}\right)$ is the adjoint operator $\pi(h)^{*}$ of $\pi(h)$, for all $h \in \mathcal{H} \rtimes \Gamma$. In particular, such representations are completely reducible.

Lemma 3.1.1. Let $\Gamma_{P}^{\prime}$ be a subgroup of $\Gamma_{P}$, let $\sigma$ be a finite dimensional $\mathcal{H}_{P} \rtimes \Gamma_{P^{-}}^{\prime}$ representation and let $t \in T^{P}$. 
(a) If $\sigma$ is unitary and $t \in T_{u n}^{P}$, then $\operatorname{Ind}_{\mathcal{H}^{P} \rtimes \Gamma_{P}^{\prime}}^{\mathcal{H} \rtimes \Gamma}\left(\sigma \circ \phi_{t}\right)$ is unitary with respect to the inner product (3.4).

(b) $\operatorname{Ind}_{\mathcal{H}^{P} \rtimes \Gamma_{P}^{\mathcal{H}} \times \Gamma_{P}^{\prime}}\left(\sigma \circ \phi_{t}\right)$ is (anti-)tempered if and only if $\sigma$ is (anti-)tempered and $t \in T_{u n}^{P}$.

Proof. Since $\Gamma$ acts by ${ }^{*}$-algebra automorphisms and $\Gamma \cdot T^{-}=T^{-}$, it does not disturb the properties unitarity and temperedness. Hence it suffices to prove the lemma in the case $\Gamma=\Gamma_{P}^{\prime}=\{$ id $\}$. Then (a) and the "if" part of (b) are Opd2, Propositions 4.19 and 4.20].

For the "only if" part of (b), suppose that $t \in T^{P} \backslash T_{u}^{P}$. Since $X \cap\left(P^{\vee}\right)^{\perp}$ is of finite index in $X^{P}=X /(X \cap \mathbb{Q} P)$, there exists $x \in X \cap\left(P^{\vee}\right)^{\perp}$ with $|t(x)| \neq 1$. Possibly replacing $x$ by $-x$, we may assume that $|t(x)|>1$. But $\delta\left(\phi_{t}\left(\theta_{x}\right)\right)(v)=x(t) v$ for all $v \in V_{\delta}$ and $x \in Z\left(X \rtimes W_{P}\right)$, so the $\mathcal{H}^{P}$-representation $\delta \circ \phi_{t}$ is not tempered. Hence its induction to $\mathcal{H}$ cannot be tempered. Similarly, if $\sigma$ is not tempered, then the restriction of $\sigma \circ \phi_{t}$ to $\mathcal{H}\left(X \cap \mathbb{Q} P, R_{P}, Y / Y \cap P^{\perp}, R_{P}^{\vee}, P, q_{P}\right)$ is not tempered.

The same proof works in the anti-tempered case, we only have to replace $|t(x)|>$ 1 by $|t(x)|<1$.

Remark. It is possible that $\operatorname{Ind}_{\mathcal{H}^{P} \rtimes \Gamma_{P}^{\prime}}^{\mathcal{H} \rtimes \Gamma}\left(\sigma \circ \phi_{t}\right)$ is unitary with respect to some inner product other than (3.4), if the conditions of part (a) are not met.

We intend to partition $\operatorname{Irr}(\mathcal{H} \rtimes \Gamma)$ into finite packets, each of which is obtained by inducing a discrete series representation of a parabolic subalgebra of $\mathcal{H}$. Thus our induction data are triples $(P, \delta, t)$, where

- $P \subset F_{0}$;

- $\left(\delta, V_{\delta}\right)$ is a discrete series representation of $\mathcal{H}_{P}$;

- $t \in T^{P}$.

Let $\Xi$ be the space of such induction data, where we regard $\delta$ only modulo equivalence of $\mathcal{H}_{P}$-representations. We say that $\xi=(P, \delta, t)$ is unitary if $t \in T_{u n}^{P}$, and we denote the space of unitary induction data by $\Xi_{u n}$. Similarly, we say that $\xi$ is positive if $|t| \in T^{P+}$, which we write as $\xi \in \Xi^{+}$. Notice that, in contrast to Langlands data, we do not require $|t|$ to be strictly positive. We have three collections of induction data:

$$
\Xi_{\text {un }} \subseteq \Xi^{+} \subseteq \Xi
$$

By default we endow these spaces with the topology for which $P$ and $\delta$ are discrete variables and $T^{P}$ carries its natural analytic topology. We will realize every irreducible $\mathcal{H} \rtimes \Gamma$-representation as a quotient of a well-chosen induced representation

$$
\pi^{\Gamma}(\xi):=\operatorname{Ind}_{\mathcal{H}}^{\mathcal{H} \rtimes \Gamma} \pi(P, \delta, t)=\operatorname{Ind}_{\mathcal{H}^{P}}^{\mathcal{H} \rtimes \Gamma}\left(\delta \circ \phi_{t}\right) .
$$

We note that for all $s \in T^{W_{0} \rtimes \Gamma}$ :

$$
\pi^{\Gamma}(P, \delta, t s)=\pi^{\Gamma}(P, \delta, t) \circ \phi_{s} .
$$

As vector space underlying $\pi^{\Gamma}(\xi)$ we will always take $\mathbb{C}\left[\Gamma W^{P}\right] \otimes V_{\delta}$. This space does not depend on $t$, which will allow us to speak of maps that are continuous, smooth, polynomial or even rational in the parameter $t \in T^{P}$.

The discrete series representations of affine Hecke algebras with irreducible root data were classified in OpSo2. Here we recall only how their central characters 
can be determined, which is related to the singularities of the elements $\imath_{s}^{0}$. Consider the $W_{0} \rtimes \Gamma$-invariant rational function

$$
\eta=\prod_{\alpha \in R_{0}} c_{\alpha}^{-1} \in \mathbb{C}(T)
$$

where $c_{\alpha}$ is as in (1.6). Notice that $\eta$ depends on the parameter function $q$, or more precisely on $q^{1 / 2}$. A coset $L$ of a subtorus of $T$ is said to be residual if the pole order of $\eta$ along $L$ equals $\operatorname{dim}_{\mathbb{C}}(T)-\operatorname{dim}_{\mathbb{C}}(L)$; see Opd4. A residual coset of dimension 0 is also called a residual point. Such points can exist only if $\mathcal{R}$ is semisimple, otherwise all residual cosets have dimension at least $\operatorname{rank}(Z(W))>0$.

According to Opd2, Lemma 3.31] the collection of central characters of discrete series representations of $\mathcal{H}(\mathcal{R}, q)$ is exactly the set of $W_{0}$-orbits of residual points for $(\mathcal{R}, q)$. Moreover, if $\delta$ is a discrete series representation of $\mathcal{H}_{P}$ with central character $W\left(R_{P}\right) r$, then $r T^{P}$ is a residual coset for $(\mathcal{R}, q)$ Opd2, Proposition 7.4]. Up to multiplication by an element of $W_{0}$, every residual coset is of this form Opd2, Proposition 7.3.v].

The map that assigns to $\xi \in \Xi$ the central character of $\pi(\xi) \in \operatorname{Mod}_{f}(\mathcal{H}(\mathcal{R}, q))$ is an algebraic morphism $\Xi \rightarrow T / W_{0}$. The above implies that the image of

$$
\left\{(P, \delta, t) \in \Xi:\left|F_{0} \backslash P\right|=d\right\}
$$

is the union of the $d$-dimensional residual cosets, modulo $W_{0}$.

Let $\delta_{\emptyset}$ be the unique onedimensional representation of $\mathcal{H}_{\emptyset}=\mathbb{C}$ and consider

$$
M(t):=\pi^{\Gamma}\left(\emptyset, \delta_{\emptyset}, t\right)=\operatorname{Ind}_{\mathcal{A}}^{\mathcal{H} \rtimes \Gamma}\left(\delta_{\emptyset} \circ \phi_{t}\right) \cong \operatorname{Ind}_{\mathcal{O}(T)}^{\mathcal{H} \rtimes \Gamma} \mathbb{C}_{t}, \quad t \in T .
$$

The family consisting of these representations is called the principal series of $\mathcal{H} \rtimes$ $\Gamma$ and is of considerable interest. For example, by Frobenius reciprocity every irreducible $\mathcal{H} \rtimes \Gamma$-representation is a quotient of some principal series representation.

Lemma 3.1.2. Suppose that $h \in \mathcal{H} \rtimes \Gamma$ and that $M(t, h)=0$ for all $t$ in some Zariski-dense subset of $T$. Then $h=0$.

Proof. Since $M(t, h) \in \operatorname{End}_{\mathbb{C}}\left(\mathbb{C}\left[\Gamma W_{0}\right]\right)$ depends algebraically on $t$, it is zero for all $t \in T$. Write $h=\sum_{\gamma w \in \Gamma \rtimes W_{0}} a_{\gamma w} N_{\gamma w}$ with $a_{\gamma w} \in \mathcal{A}$ and suppose that $h \neq 0$. Then we can find $w^{\prime} \in W_{0}$ that $a_{\gamma w^{\prime}} \neq 0$ for some $\gamma \in \Gamma$, and such that $\ell\left(w^{\prime}\right)$ is maximal for this property. From Theorem [1.2.1]d we see that

$$
M(t, h)\left(N_{e}\right)=\sum_{\gamma w \in \Gamma \rtimes W_{0}} b_{\gamma w} N_{\gamma w}
$$

for some $b_{\gamma w}$ with $b_{\gamma w^{\prime}}=a_{\gamma w^{\prime}} \neq 0$. Therefore $M(t, h)$ is not identically zero. This contradiction shows that the assumption $h \neq 0$ is untenable.

By Opd2, Corollary 2.23] discrete series representations are unitary. (Although Opdam only worked in the setting $\Gamma=\{\mathrm{id}\}$, his proof also applies with general $\Gamma$.) From this and Lemma 3.1.1 we observe:

Corollary 3.1.3. Let $\xi=(P, \delta, t) \in \Xi$. If $t \in T_{u n}^{P}$, then $\pi^{\Gamma}(\xi)$ is unitary and tempered. If $t \in T^{P} \backslash T_{u n}^{P}$, then $\pi^{\Gamma}(\xi)$ is not tempered.

For any subset $Q \subset F_{0}$, let $\Xi^{Q}, \pi^{Q}, \ldots$ denote the things $\Xi, \pi, \ldots$, but for the algebra $\mathcal{H}^{Q}$ instead of $\mathcal{H}$. For $\xi=(P, \delta, t) \in \Xi$ we define

$$
P(\xi):=\left\{\alpha \in R_{0}:|\alpha(t)|=1\right\} .
$$

Proposition 3.1.4. Let $\xi=(P, \delta, t) \in \Xi^{+}$. 
(a) The $\mathcal{H}^{P(\xi)} \rtimes \Gamma_{P(\xi)}$-representation $\pi^{P(\xi), \Gamma_{P(\xi)}}(\xi)$ is completely reducible.

(b) Every irreducible summand of $\pi^{P(\xi), \Gamma_{P(\xi)}}(\xi)$ is of the form $\pi^{P(\xi), \Gamma_{P(\xi)}}(P(\xi)$, $\left.\sigma, t^{P(\xi)}, \rho\right)$, where $\left(P(\xi), \sigma, t^{P(\xi)}, \rho\right)$ is a Langlands datum for $\mathcal{H} \rtimes \Gamma$ and $t^{P(\xi)} t^{-1} \in T_{P(\xi)}$.

(c) The irreducible quotients of $\pi^{\Gamma}(\xi)$ are the representations $L^{\Gamma}(P(\xi), \sigma$, $\left.t^{P(\xi)}, \rho\right)$, with $\left(P(\xi), \sigma, t^{P(\xi)}, \rho\right)$ coming from $(b)$.

(d) Every irreducible $\mathcal{H} \rtimes \Gamma$-representation is of the form described in (c).

(e) The functor $\operatorname{Ind}_{\mathcal{H}^{P(\xi)} \rtimes \Gamma_{P(\xi)}}^{\mathcal{H} \rtimes \Gamma}$ induces an isomorphism

$$
\operatorname{End}_{\mathcal{H}^{P(\xi)} \rtimes \Gamma_{P(\xi)}}\left(\pi^{P(\xi), \Gamma_{P(\xi)}}(\xi)\right) \cong \operatorname{End}_{\mathcal{H} \rtimes \Gamma}(\pi(\xi)) .
$$

Remarks. Part (a) holds for any $\xi \in \Xi$. In (b) $t^{P(\xi)}$ is uniquely determined modulo $K_{P(\xi)}$.

Proof. (a) By construction there exists $t^{P(\xi)} \in T^{P(\xi)}$ such that

$$
t\left(t^{P(\xi)}\right)^{-1} \in T_{P(\xi), u n}
$$

Then $\pi^{P(\xi)}(P, \delta, t) \circ \phi_{t^{P(\xi)}}^{-1}=\pi^{P(\xi)}\left(P, \delta, t\left(t^{P(\xi)}\right)^{-1}\right)$ is unitary by Corollary 3.1.3. In particular, it is completely reducible, which implies that $\pi^{P(\xi)}\left(P, \delta, t\left(t^{P(\xi)}\right)^{-1}\right)$ is also completely reducible. By [Sol5, Theorem A.1.c]

$$
\pi^{P(\xi), \Gamma_{P(\xi)}}(\xi)=\pi^{P(\xi), \Gamma_{P(\xi)}}(P, \delta, t)=\operatorname{Ind}_{\mathcal{H}^{P(\xi)}}^{\mathcal{H}^{P(\xi)} \rtimes \Gamma_{P(\xi)}} \pi^{P(\xi)}(P, \delta, t)
$$

remains completely irreducible.

(b) By Corollary $3.1 .3 \pi^{P(\xi)}\left(P, \delta, t\left(t^{P(\xi)}\right)^{-1}\right)$ is tempered and unitary, so by Lemma 2.2.6 all its irreducible summands are of the form

$$
\pi^{P(\xi)}(P(\xi), \sigma, k)=L^{P(\xi)}(P(\xi), \sigma, k) \text {, where } k \in T_{u n}^{P(\xi)} .
$$

Moreover, $\left.\pi^{P(\xi)}\left(P, \delta, t\left(t^{P(\xi)}\right)^{-1}\right)\right|_{\mathbb{C}\left[X \cap\left(P(\xi)^{\vee}\right)^{\perp}\right]}$ consists only of copies of the trivial $X \cap\left(P(\xi)^{\vee}\right)^{\perp}$-representation, so $k \in K_{P(\xi)}=T_{u n}^{P(\xi)} \cap T_{P(\xi), u n}$. Together with (3.9)

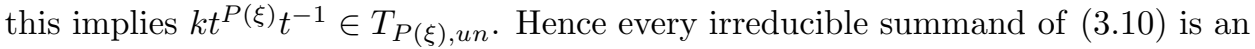
irreducible summand of some

$$
\operatorname{Ind}_{\mathcal{H}^{P(\xi)}}^{\mathcal{H}^{P(\xi)} \rtimes \Gamma_{P(\xi)}} \pi^{P(\xi)}\left(P(\xi), \sigma, k t^{P(\xi)}\right) .
$$

By Clifford theory (see the proof of Corollary 2.2.5) these are of the required form $\pi^{P(\xi), \Gamma_{P(\xi)}}\left(P(\xi), \sigma, k t^{P(\xi)}, \rho\right)$.

(c) Follows immediately from (b) and Corollary 2.2.5.

(d) By Corollary 2.2.5 b it suffices to show that every $\mathcal{H}^{P} \rtimes \Gamma_{P}$-representation of the form $\left(\sigma \circ \phi_{t}\right) \otimes \rho$ is a direct summand of some $\pi^{P, \Gamma_{P, \sigma, t}}\left(\xi^{+}\right)$. Without loss of generality, we may assume that $P=F_{0}$ and that $\Gamma_{P, \sigma, t}=\Gamma$. The $\mathcal{H}$-representation $\sigma \circ \phi_{t|t|^{-1}}$ is irreducible and tempered, so by DeOp1, Theorem 3.22] it is a direct summand of $\pi\left(\xi^{\prime}\right)$ for some $\xi^{\prime}=\left(P^{\prime}, \rho^{\prime}, t^{\prime}\right) \in \Xi_{u n}$. Then $\left(P^{\prime}, \rho^{\prime}, t^{\prime}|t|\right) \in \Xi^{+}$and $\sigma \circ \phi_{t}$ is a direct summand of $\pi\left(P^{\prime}, \rho^{\prime}, t^{\prime}|t|\right)$. By Clifford theory Sol5, Theorem A.1.b] the $\mathcal{H} \rtimes \Gamma$-representation $\left(\sigma \circ \phi_{t}\right) \otimes \rho$ is a direct summand of $\pi^{\Gamma}\left(P^{\prime}, \delta^{\prime}, t^{\prime}|t|\right)$.

(e) Follows from (a), (b) and Lemma 2.2.6 b.

The parabolically induced representations $\pi^{\Gamma}(\xi)$ are by no means all disjoint. The relations among them are described by certain intertwining operators, whose construction we recall from Opd1, Opd2. 
Suppose that $P, Q \subset F_{0}, u \in K_{P}, g \in \Gamma \ltimes W_{0}$ and $g(P)=Q$. Let $\delta$ and $\sigma$ be discrete series representations of, respectively, $\mathcal{H}_{P}$ and $\mathcal{H}_{Q}$, such that $\sigma$ is equivalent with $\delta \circ \psi_{u}^{-1} \circ \psi_{g}^{-1}$. Choose a unitary map $I_{\delta}^{g u}: V_{\delta} \rightarrow V_{\sigma}$ such that

$$
I_{\delta}^{g u}(\delta(h) v)=\sigma\left(\psi_{g} \circ \psi_{u}(h)\right)\left(I_{\delta}^{g u}(v)\right) \quad \forall v \in V_{\delta}, h \in \mathcal{H}_{P} .
$$

Notice that any two choices of $I_{\delta}^{g u}$ differ only by a complex number of norm 1 . In particular, $I_{\delta}^{g u}$ is a scalar if $\sigma=\delta$.

We obtain a bijection

$$
I_{g u}:\left(\mathbb{C}\left(T / W_{0}\right) \otimes_{Z(\mathcal{H})} \mathcal{H}\right) \rtimes \Gamma \otimes_{\mathcal{H}^{P}} V_{\delta} \rightarrow\left(\mathbb{C}\left(T / W_{0}\right) \otimes_{Z(\mathcal{H})} \mathcal{H}\right) \rtimes \Gamma \otimes_{\mathcal{H}^{Q}} V_{\sigma},
$$

$$
I_{g u}(h \otimes v)=h r_{g^{-1}}^{o} \otimes I_{\delta}^{g u}(v) .
$$

Theorem 3.1.5. (a) The map $I_{g u}$ defines an intertwining operator

$$
\pi^{\Gamma}(g u, P, \delta, t): \pi^{\Gamma}(P, \delta, t) \rightarrow \pi^{\Gamma}(Q, \sigma, g(u t)) .
$$

As a map $\mathbb{C}\left[\Gamma W^{P}\right] \otimes_{\mathbb{C}} V_{\delta} \rightarrow \mathbb{C}\left[\Gamma W^{Q}\right] \otimes_{\mathbb{C}} V_{\sigma}$ it is rational in $t \in T^{P}$ and constant on $T^{F_{0}}$-cosets.

(b) This map is regular and invertible on an open neighborhood of $T_{u n}^{P}$ in $T^{P}$ (with respect to the analytic topology).

(c) $\pi^{\Gamma}(g u, P, \delta, t)$ is unitary if $t \in T_{u n}^{P}$.

Remark. Due to the freedom in the choice of (3.11), for composable $g_{1}, g_{2} \in \mathcal{G}$ the product $\pi\left(g_{1}, g_{2} \xi\right) \pi\left(g_{2}, \xi\right)$ need not be equal to $\pi\left(g_{1} g_{2}, \xi\right)$. The difference is a locally constant function whose absolute value is 1 everywhere.

Proof. If $g=\gamma w \in \Gamma \ltimes W_{0}$, then $I_{g u}=I_{\gamma} \circ I_{w u}$, modulo this locally constant function. It follows directly from the definitions that the theorem holds for $I_{\gamma}$, so the difficult part is $I_{w u}$, which is dealt with in Opd2, Theorem 4.33 and Corollary 4.34].

The intertwining operators for reflections acting on the unitary principal series can be made reasonably explicit:

Lemma 3.1.6. Suppose that $\beta \in R_{0}$ and $t \in T_{u n}$. Then $\pi^{\Gamma}\left(s_{\beta}, \emptyset, \delta_{\emptyset}, t\right)$ is a scalar operator if and only if $c_{\beta}^{-1}(t)=0$.

Proof. Suppose that $\alpha \in F_{0}, t \in T$ and $c_{\alpha}^{-1}(t)=0$. Then 1.25) implies that $1+\imath_{s_{\alpha}}^{0}(t)=0$, regarded as an element of $\mathcal{H}\left(W_{0}, q\right)$. Hence $\pi^{\Gamma}\left(s_{\alpha}, \emptyset, \delta_{\emptyset}, t\right)$ is a scalar operator. Conversely, if $c_{\alpha}^{-1}(t) \neq 0$, then (1.25) shows that $1+\imath_{s_{\alpha}}^{0}(t)$ is not scalar, because the action of $1+q\left(s_{\alpha}\right)^{1 / 2} N_{s_{\alpha}}$ on $\mathcal{H}\left(W_{0}, q\right)$ has two different eigenvalues.

With Theorem 3.1.5 we can see that this is not specific for simple reflections. Find $w \in W_{0}$ such that $w(\beta)=\alpha$ is a simple root. Then $s_{\beta}=w^{-1} s_{\alpha} w$, so up to a nonzero scalar

$$
\pi^{\Gamma}\left(s_{\beta}, \emptyset, \delta_{\emptyset}, t\right)=\pi^{\Gamma}\left(w^{-1}, \emptyset, \delta_{\emptyset}, w t\right) \pi^{\Gamma}\left(s_{\alpha}, \emptyset, \delta_{\emptyset}, w t\right) \pi^{\Gamma}\left(w, \emptyset, \delta_{\emptyset}, t\right) .
$$

Now we notice that $c_{\beta}^{-1}(t)=0$ if and only if $c_{\alpha}^{-1}(w t)=0$, and that $\pi^{\Gamma}\left(w^{-1}, \emptyset, \delta_{\emptyset}, w t\right)$ $=\pi^{\Gamma}\left(w, \emptyset, \delta_{\emptyset}, t\right)^{-1}$ up to a scalar.

Thus it is possible to determine the $\mathcal{H}$-endomorphisms for unitary principal series representations, at least when the isotropy groups of points $t \in T_{u n}$ are generated by reflections. The reducibility and intertwining operators for nonunitary principal series are more complicated, and have been subjected to ample study Kat1, Rog, 
Ree1. For other parabolically induced representations the intertwining operators are less explicit. They can be understood better with the theory of R-groups DeOp2.

The action of these intertwining operators on the induction data space $\Xi$ is described most conveniently with a groupoid $\mathcal{G}$ that includes all pairs $(g, u)$ as above. The base space of $\mathcal{G}$ is the power set of $F_{0}$, and for $P, Q \subseteq F_{0}$ the collection of arrows from $P$ to $Q$ is

$$
\mathcal{G}_{P Q}=\left\{(g, u): g \in \Gamma \ltimes W_{0}, u \in K_{P}, g(P)=Q\right\} .
$$

Whenever it is defined, the multiplication in $\mathcal{G}$ is

$$
\left(g^{\prime}, u^{\prime}\right) \cdot(g, u)=\left(g^{\prime} g, g^{-1}\left(u^{\prime}\right) u\right) .
$$

Usually we will write elements of $\mathcal{G}$ simply as $g u$. This groupoid acts from the left on $\Xi$ by

$$
(g, u) \cdot(P, \delta, t):=\left(g(P), \delta \circ \psi_{u}^{-1} \circ \psi_{g}^{-1}, g(u t)\right),
$$

the action being defined if and only if $g(P) \subset F_{0}$. Since $T^{+} \supset T^{P+}$ is a fundamental domain for the action of $W_{0}$ on $T$, every element of $\Xi$ is $\mathcal{G}$-associate to an element of $\Xi^{+}$.

Although $\pi^{\Gamma}(g u(P, \delta, t))$ and $\pi^{\Gamma}(P, \delta, t)$ are not always isomorphic, the existence of rational intertwining operators has the following consequence:

Lemma 3.1.7. The $\mathcal{H} \rtimes \Gamma$-representations $\pi^{\Gamma}(g u(P, \delta, t))$ and $\pi^{\Gamma}(P, \delta, t)$ have the same irreducible subquotients, counted with multiplicity.

Proof. This is not hard, the proof in the graded Hecke algebra setting [Sol5, Lemma 3.4 ] also works here.

3.2. The Schwartz algebra. We recall the construction of various topological completions of $\mathcal{H} \mathrm{Opd2}$ : a Hilbert space, a $C^{*}$-algebra and a Schwartz algebra. The latter is the most relevant from the representation theoretic point of view. All tempered representations of $\mathcal{H}$ extend to its Schwartz completion, and a close study of this Schwartz algebra reveals facts about tempered representations for which no purely algebraic proof is known.

Let $L^{2}(\mathcal{R}, q)$ be Hilbert space completion of $\mathcal{H}$ with respect to the inner product (3.2). By means of the orthonormal basis $\left\{N_{w}: w \in W\right\}$ we can identify $L^{2}(\mathcal{R}, q)$ with the Hilbert space $L^{2}(W)$ of square integrable functions $W \rightarrow \mathbb{C}$.

For any $h \in \mathcal{H}$ the map $\mathcal{H}(\mathcal{R}, q) \rightarrow \mathcal{H}(\mathcal{R}, q): h^{\prime} \mapsto h h^{\prime}$ extends to a bounded linear operator on $L^{2}(\mathcal{R}, q)$. This realizes $\mathcal{H}(\mathcal{R}, q)$ as a *-subalgebra of $B\left(L^{2}(\mathcal{R}, q)\right)$. Its closure $C^{*}(\mathcal{R}, q)$ is a separable unital $C^{*}$-algebra, called the $C^{*}$-algebra of $\mathcal{H}$.

The Schwartz completion of $\mathcal{H}$ will, as a topological vector space, consist of all rapidly decaying functions on $W$, with respect to some length function. For this purpose the length function $\ell(w)$ of the Coxeter system ( $\left.W^{\text {aff }}, S^{\text {aff }}\right)$ is unsatisfactory, because its natural extension to $W$ is zero on $Z(W)$. To overcome this inconvenience, recall that

$$
X \otimes_{\mathbb{Z}} \mathbb{R}=\mathfrak{a}^{*}=\mathfrak{a}_{F_{0}}^{*} \oplus \mathfrak{a}^{* F_{0}}=\mathfrak{a}_{F_{0}}^{*} \oplus\left(Z(W) \otimes_{\mathbb{Z}} \mathbb{R}\right) .
$$

Thus we can decompose any $x \in X \subset \mathfrak{a}^{*}$ uniquely as $x=x_{F_{0}}+x^{F_{0}} \in \mathfrak{a}_{F_{0}}^{*} \oplus \mathfrak{a}^{* F_{0}}$. Now we define

$$
\mathcal{N}(w)=\ell(w)+\left\|w(0)^{F_{0}}\right\| \quad w \in W .
$$


Since $W^{\text {aff }} \oplus Z(W)$ is of finite index in $W$, the set $\{w \in W: \mathcal{N}(w)=0\}$ is finite. For $n \in \mathbb{N}$ we define the following norm on $\mathcal{H}$ :

$$
p_{n}\left(\sum_{w \in W} h_{w} N_{w}\right)=\sup _{w \in W}\left|h_{w}\right|(\mathcal{N}(w)+1)^{n} .
$$

The completion $\mathcal{S}=\mathcal{S}(\mathcal{R}, q)$ of $\mathcal{H}$ with respect to the family of norms $\left\{p_{n}: n \in \mathbb{N}\right\}$ is a nuclear Fréchet space. It consists of all (possibly infinite) sums $h=\sum_{w \in W} h_{w} N_{w}$ such that $p_{n}(h)<\infty$ for all $n \in \mathbb{N}$.

Theorem 3.2.1. There exist $C_{q}>0, d \in \mathbb{N}$ such that $\forall h, h^{\prime} \in \mathcal{S}(\mathcal{R}, q), n \in \mathbb{N}$,

$$
\begin{aligned}
& \|h\|_{B\left(L^{2}(\mathcal{R}, q)\right)} \leq C_{q} p_{d}(h), \\
& p_{n}\left(h \cdot h^{\prime}\right) \leq C_{q} p_{n+d}(h) p_{n+d}\left(h^{\prime}\right) .
\end{aligned}
$$

In particular, $\mathcal{S}(\mathcal{R}, q)$ is a unital locally convex ${ }^{*}$-algebra, and it is contained in $C^{*}(\mathcal{R}, q)$.

Proof. This was proven first with representation theoretic methods in Opd2, Section 6.2]. Later the author found a purely analytic proof [OpSo2, Theorem A.7].

It is easily seen that the action of $\Gamma$ on $\mathcal{H}$ preserves all the above norms. Hence the crossed product $\mathcal{S} \rtimes \Gamma=\mathcal{S}(\mathcal{R}, q) \rtimes \Gamma$ (respectively $\left.C^{*}(\mathcal{R}, q) \rtimes \Gamma\right)$ is a well-defined Fréchet algebra (respectively $C^{*}$-algebra). For $q=1$ we obtain the algebras,

$$
\begin{aligned}
& \mathcal{S}(\mathcal{R}, 1) \rtimes \Gamma=\mathcal{S}\left(W^{\prime}\right)=\mathcal{S}(X) \rtimes W_{0}^{\prime}=C^{\infty}\left(T_{\text {un }}\right) \rtimes W_{0}^{\prime}, \\
& C^{*}(\mathcal{R}, 1) \rtimes \Gamma=C^{*}\left(W^{\prime}\right)=C^{*}(X) \rtimes W_{0}^{\prime}=C\left(T_{\text {un }}\right) \rtimes W_{0}^{\prime},
\end{aligned}
$$

where $\mathcal{S}(X)$ denotes the algebra of rapidly decreasing functions on $X$.

We can use these topological completions to characterize discrete series and tempered representations. According to Opd2, Lemma 2.22], an irreducible $\mathcal{H} \rtimes \Gamma$ representation $\pi$ is discrete series if and only if it is contained in the left regular representation of $\mathcal{H} \rtimes \Gamma$ on $L^{2}(\mathcal{R}, q) \otimes \mathbb{C}[\Gamma]$, or equivalently if its character $\chi_{\pi}$ : $\mathcal{H} \rtimes \Gamma \rightarrow \mathbb{C}$ extends to a continuous linear functional on $L^{2}(\mathcal{R}, q) \otimes \mathbb{C}[\Gamma]$.

By Opd2, Lemma 2.20] a finite dimensional $\mathcal{H} \rtimes \Gamma$-representation is tempered if and only if it extends continuously to an $\mathcal{S} \rtimes \Gamma$-representation. More generally, suppose that $\pi$ is a representation of $\mathcal{H} \rtimes \Gamma$ on a Fréchet space $V$, possibly of infinite dimension. As in OpSo1, Proposition A.2], we define $\pi$ to be tempered if it induces a continuous map $(\mathcal{S} \rtimes \Gamma) \times V \rightarrow V$.

A crucial role in the harmonic analysis on affine Hecke algebra is played by a particular Fourier transform, which is based on the induction data space $\Xi$. Let $\mathcal{V} \Xi$ be the vector bundle over $\Xi$, whose fiber at $(P, \delta, t) \in \Xi$ is the representation space $\mathbb{C}\left[\Gamma \times W^{P}\right] \otimes V_{\delta}$ of $\pi^{\Gamma}(P, \delta, t)$. Let $\operatorname{End}\left(\mathcal{V}_{\Xi}^{\Gamma}\right)$ be the algebra bundle with fibers $\operatorname{End}_{\mathbb{C}}\left(\mathbb{C}\left[\Gamma \times W^{P}\right] \otimes V_{\delta}\right)$. The inner product (3.4) endows $\operatorname{End}_{\mathbb{C}}\left(\mathbb{C}\left[\Gamma \times W^{P}\right] \otimes V_{\delta}\right)$ and $\operatorname{End}\left(\mathcal{V}_{\Xi}^{\Gamma}\right)$ with a canonical involution *. Of course these vector bundles are trivial on every connected component of $\Xi$, but globally not even the dimensions need be constant. Since $\Xi$ has the structure of a complex algebraic variety, we can construct the algebra of polynomial sections of $\operatorname{End}\left(\mathcal{V}_{\Xi}^{\Gamma}\right)$ :

$$
\mathcal{O}\left(\Xi ; \operatorname{End}\left(\mathcal{V}_{\Xi}^{\Gamma}\right)\right):=\bigoplus_{P, \delta} \mathcal{O}\left(T^{P}\right) \otimes \operatorname{End}_{\mathbb{C}}\left(\mathbb{C}\left[\Gamma \times W^{P}\right] \otimes V_{\delta}\right) .
$$

Given a reasonable subset (preferably a submanifold) $\Xi^{\prime} \subset \Xi$, we define the algebras $L^{2}\left(\Xi^{\prime} ; \operatorname{End}\left(\mathcal{V}_{\Xi}^{\Gamma}\right)\right), C\left(\Xi^{\prime} ; \operatorname{End}\left(\mathcal{V}_{\Xi}^{\Gamma}\right)\right)$ and $C^{\infty}\left(\Xi^{\prime} ; \operatorname{End}\left(\mathcal{V}_{\Xi}^{\Gamma}\right)\right)$ in similar fashion. 
Furthermore, if $\mu$ is a sufficiently nice measure on $\Xi$ and $\Xi^{\prime}$ is compact, then the following formula defines a Hermitian form on $L^{2}\left(\Xi^{\prime} ; \operatorname{End}\left(\mathcal{V}_{\Xi}^{\Gamma}\right)\right)$ :

$$
\left\langle f_{1}, f_{2}\right\rangle_{\mu}:=\int_{\Xi^{\prime}} \operatorname{tr}\left(f_{1}(\xi)^{*} f_{2}(\xi)\right) \mathrm{d} \mu .
$$

The intertwining operators from Theorem 3.1.5 give rise to an action of the groupoid $\mathcal{G}$ on the algebra of rational sections of $\operatorname{End}\left(\mathcal{V}_{\Xi}^{\Gamma}\right)$, by

$$
(g \cdot f)(\xi)=\pi^{\Gamma}\left(g, g^{-1} \xi\right) f\left(g^{-1} \xi\right) \pi^{\Gamma}\left(g, g^{-1} \xi\right)^{-1},
$$

whenever $g^{-1} \xi \in \Xi$ is defined. This formula also defines groupoid actions of $\mathcal{G}$ on $C\left(\Xi^{\prime} ; \operatorname{End}\left(\mathcal{V}_{\Xi}^{\Gamma}\right)\right)$ and on $C^{\infty}\left(\Xi^{\prime} ; \operatorname{End}\left(\mathcal{V}_{\Xi}^{\Gamma}\right)\right)$, provided that $\Xi^{\prime}$ is a $\mathcal{G}$-stable submanifold of $\Xi$ on which all the intertwining operators are regular. Given a suitable collection $\Sigma$ of sections of $\left(\Xi^{\prime}, \operatorname{End}\left(\mathcal{V}_{\Xi}^{\Gamma}\right)\right)$, we write

$$
\Sigma^{\mathcal{G}}=\left\{f \in \Sigma:(g \cdot f)(\xi)=f(\xi) \text { for all } g \in \mathcal{G}, \xi \in \Xi^{\prime} \text { such that } g^{-1} \xi \text { is defined }\right\} .
$$

The Fourier transform for $\mathcal{H} \rtimes \Gamma$ is the algebra homomorphism

$$
\begin{aligned}
& \mathcal{F}: \mathcal{H} \rtimes \Gamma \rightarrow \mathcal{O}\left(\Xi ; \operatorname{End}\left(\mathcal{V}_{\Xi}^{\Gamma}\right)\right), \\
& \mathcal{F}(h)(\xi)=\pi(\xi)(h) .
\end{aligned}
$$

The very definition of intertwining operators shows that the image of $\mathcal{F}$ is contained in the algebra $\mathcal{O}\left(\Xi ; \operatorname{End}\left(\mathcal{V}_{\Xi}^{\Gamma}\right)\right)^{\mathcal{G}}$. The Fourier transform also extends continuously to various topological completions of $\mathcal{H} \rtimes \Gamma$ :

Theorem 3.2.2 (Plancherel theorem for affine Hecke algebras). The Fourier transform induces algebra homomorphisms:

$$
\begin{array}{rrr}
\mathcal{H}(\mathcal{R}, q) \rtimes \Gamma & \rightarrow & \mathcal{O}(\Xi ; \operatorname{End}(\mathcal{V} \Xi))^{\mathcal{G}}, \\
\mathcal{S}(\mathcal{R}, q) \rtimes \Gamma & \rightarrow & C^{\infty}\left(\Xi_{u n} ; \operatorname{End}(\mathcal{V} \Xi)\right)^{\mathcal{G}}, \\
C^{*}(\mathcal{R}, q) \rtimes \Gamma & \rightarrow & C\left(\Xi_{\text {un }} ; \operatorname{End}(\mathcal{V} \Xi)\right)^{\mathcal{G}} .
\end{array}
$$

The first one is injective, the second is an isomorphism of Fréchet ${ }^{*}$-algebras and the third is an isomorphism of $C^{*}$-algebras.

Furthermore, there exists a unique Plancherel measure $\mu_{P l}$ on $\Xi$ such that

- the support of $\mu_{P l}$ is $\Xi_{u n}$;

- $\mu_{P l}$ is $\mathcal{G}$-invariant;

- the restriction of $\mu_{p l}$ to a component $\left(P, \delta, T^{P}\right)$ is absolutely continuous with respect to the Haar measure of $T_{u n}^{P}$;

- the Fourier transform extends to a bijective isometry

$$
\left(L^{2}(\mathcal{R}, q) \otimes \mathbb{C}[\Gamma] ;\langle,\rangle_{\tau}\right) \rightarrow\left(L^{2}\left(\Xi_{\text {un }} ; \operatorname{End}\left(\mathcal{V}_{\Xi}^{\Gamma}\right)\right)^{\mathcal{G}} ;\langle,\rangle_{\mu_{P l}}\right) .
$$

Proof. Once again the essential case is $\Gamma=\{\mathrm{id}\}$, which is a very deep result proven by Delorme and Opdam; see Theorem 5.3 and Corollary 5.7 of DeOp1 and Opd2, Theorem 4.43].

To include $\Gamma$ in the picture we need a result of general nature. Let $A$ be any complex $\Gamma$-algebra and endow $A \otimes_{\mathbb{C}} \operatorname{End}(\mathbb{C}[\Gamma])$ with the $\Gamma$-action

$$
\gamma \cdot(a \otimes f)(v)=\gamma \cdot a \otimes f(v \gamma) \gamma^{-1} \quad a \in A, \gamma \in \Gamma, v \in \mathbb{C}[\Gamma], f \in \operatorname{End}(\mathbb{C}[\Gamma]) .
$$

There is a natural isomorphism

$$
A \rtimes \Gamma \cong\left(A \otimes_{\mathbb{C}} \operatorname{End}(\mathbb{C}[\Gamma])\right)^{\Gamma} .
$$


This is easy to show, but it appears to be one of those folklore results whose origins are hard to retrace. In any case a proof can be found in [Sol3, Lemma A.3]. For $A=C\left(\Xi_{\text {un }} ; \operatorname{End}\left(\mathcal{V}_{\Xi}^{\Gamma}\right)\right)$ the action (3.17) corresponds to the action of $\Gamma$ on $\operatorname{End}\left(\mathcal{V}_{\Xi}^{\Gamma}\right)$ described in (3.16). The greater part of the theorem follows from (3.18) and the case $\Gamma=\{\mathrm{id}\}$. It only remains to see how the inner products $\langle,\rangle_{\tau}$ and $\langle,\rangle_{\mu_{P l}}$ behave when $\Gamma$ is included. Let us distinguish the new inner products with a subscript $\Gamma$. On the Hecke algebra side it is easy, as formula (3.1) does not change, so

$$
\left\langle N_{\gamma} h, N_{\gamma^{\prime}} h^{\prime}\right\rangle_{\Gamma, \tau}=\left\{\begin{array}{lll}
\left\langle h, h^{\prime}\right\rangle_{\tau} & \text { if } \gamma=\gamma^{\prime}, \\
0 & \text { if } \gamma \neq \gamma^{\prime}
\end{array}\right.
$$

On the spectral side the inclusion of $\Gamma$ means that we replace every $\mathcal{H}$-representation $\pi(\xi)$ by $\operatorname{Ind}_{\mathcal{H}}^{\mathcal{H} \rtimes \Gamma} \pi(\xi)$. In such an induced representation the elements of $\Gamma$ permute the $\mathcal{H}$-subrepresentations $\gamma \pi(\xi)$, while $h \in \mathcal{H}$ acts by $\pi\left(\gamma^{-1}(h)\right)$ on $\gamma \pi(\xi)$. The action of $\Gamma$ on $\mathcal{H}$ preserves the trace and the ${ }^{*}$, so

$$
\operatorname{tr}\left(\pi^{\Gamma}\left(\xi, N_{\gamma} h\right)^{*} \pi^{\Gamma}\left(\xi, N_{\gamma^{\prime}} h^{\prime}\right)\right)= \begin{cases}\operatorname{tr}\left(\pi(\xi, h)^{*} \pi\left(\xi, h^{\prime}\right)\right) & \text { if } \gamma=\gamma^{\prime}, \\ 0 & \text { if } \gamma \neq \gamma^{\prime} .\end{cases}
$$

In view of (3.15), this means that the $L^{2}$-extension of $\mathcal{F}$ is an isometry with respect to the Plancherel measure $\mu_{\Gamma, P l}=|\Gamma|^{-1} \mu_{P l}$.

Corollary 3.2.3. The center of $\mathcal{S}(\mathcal{R}, q) \rtimes \Gamma$ (respectively $\left.C^{*}(\mathcal{R}, q) \rtimes \Gamma\right)$ is isomorphic to $C^{\infty}\left(\Xi_{u n}\right)^{\mathcal{G}}$ (respectively $\left.C\left(\Xi_{u n}\right)^{\mathcal{G}}\right)$.

Proof. This is the obvious generalization of DeOp1, Corollary 5.5] to our setting.

Notice that $Z(\mathcal{S} \rtimes \Gamma)$ is larger than the closure of $Z(\mathcal{H} \rtimes \Gamma)$ in $\mathcal{S} \rtimes \Gamma$, for example, $Z(\mathcal{S} \rtimes \Gamma)$ contains a nontrivial idempotent for every connected component of $\Xi_{\text {un }} / \mathcal{G}$. Varying on the notation $\operatorname{Mod}_{f, U}(\mathcal{H} \rtimes \Gamma)$ we will denote by

$$
\operatorname{Mod}_{f, \Sigma}(\mathcal{S} \rtimes \Gamma)
$$

the category of finite dimensional $\mathcal{S} \rtimes \Gamma$-modules with $Z(\mathcal{S} \rtimes \Gamma)$-weights in $\Sigma \subset$ $\Xi_{\text {un }} / \mathcal{G}$.

Let us compare Schwartz algebras of affine Hecke algebras with those for reductive $p$-adic groups. Suppose that $G$ is reductive $p$-adic group and that $\mathcal{H} \rtimes \Gamma$ is Morita equivalent to $\mathcal{H}(G)_{\mathfrak{s}}$, in the notation of Section 1.6. The (conjectural) isomorphism described in (1.28) is such that $\mathfrak{a}^{*}=X \otimes_{\mathbb{Z}} \mathbb{R}$ corresponds to $X_{*}(A) \otimes_{\mathbb{Z}} \mathbb{R}$. The conditions for temperedness of finite length representations of $\mathcal{H} \rtimes \Gamma$ and $\mathcal{H}(G)_{\mathfrak{s}}$ are formulated in terms of corresponding negative cones in $\mathfrak{a}^{*}$ and in $X_{*}(A) \otimes_{\mathbb{Z}} \mathbb{R}$. Therefore such a Morita equivalence would preserve temperedness of representations. Thus $\operatorname{Mod}_{f}(\mathcal{S} \rtimes \Gamma)$ would be equivalent to the category of finite length modules

$$
\operatorname{Mod}_{f, \mathfrak{s}}(\mathcal{S}(G))=\operatorname{Mod}_{f}\left(\mathcal{S}(G)_{\mathfrak{s}}\right),
$$

where $\mathcal{S}(G)$ is the Harish-Chandra-Schwartz algebra of $G$ and $\mathcal{S}(G)_{\mathfrak{s}}$ is its two-sided ideal corresponding to the inertial equivalence class $\mathfrak{s} \in \mathfrak{B}(G)$.

Moreover, $I$ is an Iwahori subgroup of a split group $G$, it is shown in DeOp1, Proposition 10.2] that the isomorphism $\mathcal{H}(G, I) \cong \mathcal{H}(\mathcal{R}, q)$ extends to an isomorphism $\mathcal{S}(G, I) \cong \mathcal{S}(\mathcal{R}, q)$. Therefore it is reasonable to expect that more generally $\mathcal{S}(G)_{\mathfrak{s}}$ will be Morita equivalent to $\mathcal{S}(\mathcal{R}, q) \rtimes \Gamma$ in case of an isomorphism (1.28). Further support of this is provided by Theorem 3.2 .2 in comparison with the Plancherel 
theorem for $\mathcal{S}(G)$ Wal and for $C_{r}^{*}(G)$ Ply2. These show that $\mathcal{S}(\mathcal{R}, q) \rtimes \Gamma$ and $\mathcal{S}(G)_{\mathfrak{s}}$, as well as their respective $C^{*}$-completions, have a very similar shape, which can almost entirely be deduced from their categories of finite length modules.

3.3. Parametrization of representations with induction data. Theorem 3.2 .2 is extremely deep and useful, a large part of what follows depends on it. It shows a clear advantage of $\mathcal{S}$ over $\mathcal{H}$, namely that the Fourier transform consists of all smooth sections. In particular one can use any smooth section, without knowing its preimage under $\mathcal{F}$. By Corollary 3.2 .3 the irreducible tempered $\mathcal{H} \rtimes \Gamma$ representations are partitioned in finite packets parametrized by $\Xi_{u n} / \mathcal{G}$. Moreover, from Theorem 3.2.2 Delorme and Opdam also deduce analogues of HarishChandra's Completeness Theorem DeOp1, Corollary 5.4] and of Langlands' Disjointness Theorem [DeOp1, Corollary 5.8].

We will generalize these results to all irreducible representations. For that we do not need all induction data from $\Xi$, in view of Lemma 3.1.7 it suffices to consider $\xi \in \Xi^{+}$. At the same time this restriction to positive induction data enables us to avoid the singularities of the intertwiners $\pi(g, \xi)$.

Theorem 3.3.1. Let $\xi=(P, \delta, t), \xi^{\prime}=\left(P^{\prime}, \delta \frac{1}{2}, t^{\prime}\right) \in \Xi^{+}$.

(a) The $\mathcal{H} \rtimes \Gamma$-representations $\pi^{\Gamma}(\xi)$ and $\pi^{\Gamma}\left(\xi^{\prime}\right)$ have a common irreducible quotient if and only if there exists a $g \in \mathcal{G}$ such that $g \xi=\xi^{\prime}$.

(b) The operators $\left\{\pi^{\Gamma}(g, \xi): g \in \mathcal{G}, g \xi=\xi^{\prime}\right\}$ are regular and invertible, and they span $\operatorname{Hom}_{\mathcal{H} \rtimes \Gamma}\left(\pi^{\Gamma}(\xi), \pi^{\Gamma}\left(\xi^{\prime}\right)\right)$.

Proof. (a) This result is analogous to [Sol5, Theorem 8.2] in the case of graded Hecke algebras, and for the "if" part the proof is also the same. The "only if" direction is somewhat more complicated, since replacing $\xi$ by its unitary part is too simple.

Suppose that $\pi^{\Gamma}(\xi)$ and $\pi^{\Gamma}\left(\xi^{\prime}\right)$ have a common irreducible quotient. Since $\pi^{\Gamma}\left(\gamma, \xi^{\prime}\right)$ is invertible for any $\gamma \in \Gamma$, we may replace $\xi^{\prime}$ by $\gamma \xi^{\prime}$. In view of this, Proposition 3.1.4 c and Corollary 2.2.5. b we may assume that $P(\xi)=P\left(\xi^{\prime}\right)$ and that the $\mathcal{H}^{P(\xi)} \rtimes \Gamma_{P(\xi)}$-representations $\pi^{P(\xi), \Gamma_{P(\xi)}}(\xi)$ and $\pi^{P(\xi), \Gamma_{P(\xi)}\left(\xi^{\prime}\right)}$ have a common irreducible summand $\pi^{P(\xi), \Gamma_{P(\xi)}}\left(P(\xi), \sigma, t^{P(\xi)}, \rho\right)$.

Choose $s \in T_{u n}^{P(\xi)} t^{P(\xi)}$ such that $t^{P(\xi)}$ and $t^{P(\xi)} s^{-1}$ have the same isotropy group in $W_{0} \rtimes \Gamma$. This is possible because $T^{g}$ is a complex algebraic subtorus of $T$ for every $g \in W_{0} \rtimes \Gamma$. The $\mathcal{H}^{P(\xi)} \rtimes \Gamma_{P(\xi)}$-representations $\pi^{P(\xi), \Gamma_{P(\xi)}}\left(P, \delta, t s^{-1}\right)$ and $\pi^{P(\xi), \Gamma_{P(\xi)}}\left(P^{\prime}, \delta^{\prime}, t^{\prime} s^{-1}\right)$ are completely reducible by Proposition 3.1.4 a, and they have the common irreducible summand

$$
\pi^{P(\xi), \Gamma_{P(\xi)}}\left(P(\xi), \sigma, t^{P(\xi)} s^{-1}, \rho\right)=\operatorname{Ind}_{\mathcal{H}^{P(\xi)} \rtimes \Gamma_{P(\xi), \sigma, t^{P(\xi)}}}^{\mathcal{H}^{P(\xi)} \rtimes \Gamma_{P(\xi)}}\left(\sigma \circ \phi_{t^{P(\xi)}} \circ \phi_{s}^{-1} \otimes \rho\right) .
$$

Moreover, because every irreducible summand is of this form,

$$
\begin{aligned}
\operatorname{Hom}_{\mathcal{H}^{P(\xi)} \rtimes \Gamma_{P(\xi)}}\left(\pi^{P(\xi), \Gamma_{P(\xi)}}\left(P, \delta, t s^{-1}\right), \pi^{P(\xi), \Gamma_{P(\xi)}}\left(P^{\prime}, \delta^{\prime}, t^{\prime} s^{-1}\right)\right) \\
\cong \operatorname{Hom}_{\mathcal{H}^{P(\xi)} \rtimes \Gamma_{P(\xi)}}\left(\pi^{P(\xi), \Gamma_{P(\xi)}}(P, \delta, t), \pi^{P(\xi), \Gamma_{P(\xi)}}\left(P^{\prime}, \delta^{\prime}, t^{\prime}\right)\right) \neq 0 .
\end{aligned}
$$

Since $t^{P(\xi)} s^{-1} \in T_{u n}$, we have $t s^{-1}, t^{\prime} s^{-1} \in T_{u n}$. So $|t|=\left|t^{\prime}\right|$ and the representations $\pi^{P(\xi), \Gamma_{P(\xi)}}\left(P, \delta, t s^{-1}\right)$ and $\pi^{P(\xi), \Gamma_{P(\xi)}}\left(P^{\prime}, \delta^{\prime}, t^{\prime} s^{-1}\right)$ extend continuously to $\mathcal{S}\left(\mathcal{R}^{P(\xi)}, q^{P(\xi)}\right) \rtimes \Gamma_{P(\xi)}$. Now Theorem 3.2.2 for this algebra shows that the left-hand side of (3.20) is spanned by the intertwiners $\pi^{P(\xi), \Gamma_{P(\xi)}}\left(g, P, \delta, t s^{-1}\right)$ with 
$g\left(P, \delta, t s^{-1}\right)=\left(P^{\prime}, \delta^{\prime}, t^{\prime} s^{-1}\right)$. Since (3.20) is nonzero, there exists at least one such $g \in \mathcal{G}$. The choice of $s$ guarantees that $g(P, \delta, t)=\left(P^{\prime}, \delta^{\prime}, t^{\prime}\right)$ as well.

(b) By Theorem 3.1.5 the $\pi^{P(\xi), \Gamma_{P(\xi)}}\left(g, P, \delta, t s^{-1}\right)$ are invertible and constant on $T^{P(\xi)}$-cosets. Hence the $\pi^{P(\xi), \Gamma_{P(\xi)}}(g, P, \delta, t)$ span the right-hand side of (3.20), and they are invertible.

It is interesting to compare Theorem 3.3.1 with [Ree1, which describes the $\mathcal{H}$-endomorphisms of principal series representations $M(t)$. It transpires that the results of [Ree1] simplify considerably when $|t|$ is in the positive Weyl chamber; then $\operatorname{End}_{\mathcal{H}}(M(t))$ is semisimple and all its irreducible quotients occur with multiplicity one.

Now we can prove the desired partition of $\operatorname{Irr}(\mathcal{H} \rtimes \Gamma)$ in packets:

Theorem 3.3.2. Let $\pi$ be an irreducible $\mathcal{H} \rtimes \Gamma$-representation. There exists a unique association class $\mathcal{G}(P, \delta, t) \in \Xi / \mathcal{G}$ such that the following equivalent properties hold:

(a) $\pi$ is isomorphic to an irreducible quotient of $\pi^{\Gamma}\left(\xi^{+}\right)$, for some $\xi^{+} \in \Xi^{+} \cap$ $\mathcal{G}(P, \delta, t)$

(b) $\pi$ is a constituent of $\pi^{\Gamma}(P, \delta, t)$, and $\left\|c c_{P}(\delta)\right\|$ is maximal for this property.

Proof. This is the analogue of [Sol5, Theorem 8.3]. Proposition 3.1.4.d says that there exists $\xi^{+}=\left(P^{\prime}, \delta^{\prime}, t^{\prime}\right) \in \Xi^{+}$satisfying (a), and by Theorem 3.3 .1 its $\mathcal{G}$ association class is unique. The remainder of the proof is just as for graded Hecke algebras, as in Sol5. The crucial ingredient is Lemma 2.2.6. b.

All of these constructs with induction data have direct analogues in the setting of graded Hecke algebras [Sol5, Sections 6 and 8]. Concretely, $\tilde{\Xi}$ is the space of all triples $\tilde{\xi}=(Q, \sigma, \lambda)$, where $Q \subset F_{0}, \sigma$ is a discrete series representation of $\mathbb{H}_{Q}$ and $\lambda \in \mathfrak{t}^{Q}$. The subsets of unitary (respectively positive) induction data are obtained by imposing the restriction $\lambda \in i \mathfrak{a}^{Q}$ (respectively $\lambda \in \mathfrak{a}^{Q+}+i \mathfrak{a}^{Q}$ ). The corresponding induced representation is

$$
\pi^{\Gamma}(\tilde{\xi})=\operatorname{Ind}_{\mathbb{H}}^{\mathbb{H} \rtimes \Gamma} \pi(Q, \sigma, \lambda)=\operatorname{Ind}_{\mathbb{H}_{Q}}^{\mathbb{H} \rtimes \Gamma}\left(\sigma_{\lambda}\right) .
$$

The groupoid $\tilde{\mathcal{G}}$ and its action on $\tilde{\Xi}$ are defined like $\mathcal{G}$, but without the parts $K_{P}$. We would like to understand the relation between induction data for $\mathcal{H} \rtimes \Gamma$ and for $\mathbb{H} \rtimes \Gamma$. We consider, for every $u \in T_{u n}$, the induction data for $\left(\tilde{\mathcal{R}}_{u}, k_{u}\right)$ with $\lambda \in \mathfrak{a}$. Thus we arrive at the space $\widehat{\Xi}$ of quadruples $\hat{\xi}=(u, \tilde{P}, \tilde{\delta}, \lambda)$ such that:

- $u \in T_{u n}$;

- $\tilde{P} \subset F_{u}$;

- $\tilde{\delta}$ is a discrete series representation of $\mathbb{H}\left(\tilde{\mathcal{R}}_{u, \tilde{P}}, k_{u, \tilde{P}}\right)$;

- $\lambda \in \mathfrak{a}^{\tilde{P}}$.

The $\mathcal{H} \rtimes \Gamma$-representation associated to $\hat{\xi}$ is

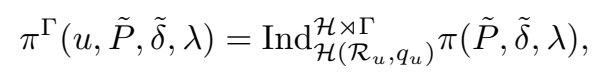

where the $\mathbb{H}\left(\tilde{\mathcal{R}}_{u}, k_{u}\right)$-representation $\pi(\tilde{P}, \tilde{\delta}, \lambda)$ is considered as a representation of $\mathcal{H}\left(\tilde{\mathcal{R}}_{u}, q_{u}\right)$, via Theorem 2.1.4.

For $g \in \mathcal{G}$ the map $\psi_{g}$ from (1.21) and (1.17) induces an algebra isomorphism $\mathbb{H}\left(\tilde{\mathcal{R}}_{u}, k_{u}\right) \rightarrow \mathbb{H}\left(\tilde{\mathcal{R}}_{g(u)}, k_{g(u)}\right)$, and the stabilizer in $\mathcal{G}$ of $u \in T_{u n}$ is the groupoid $\tilde{\mathcal{G}}_{u}$ associated to $\left(\tilde{\mathcal{R}}_{u}, k_{u}\right)$. This leads to an action of $\mathcal{G}$ on $\widehat{\Xi}$. 
The collections of $\mathcal{H} \rtimes \Gamma$-representations corresponding to $\Xi$ and to $\widehat{\Xi}$ are almost the same, but not entirely:

Lemma 3.3.3. There exists a natural finite-to-one surjection

$$
\Xi / \mathcal{G} \rightarrow \widehat{\Xi} / \mathcal{G}, \mathcal{G} \xi \mapsto \mathcal{G} \hat{\xi}
$$

with the following property. Given $\hat{\xi} \in \widehat{\Xi}$ one can find $\xi_{i} \in \Xi$ (not necessarily all different) such that $\bigcup_{i} \mathcal{G} \xi_{i}$ is the preimage of $\mathcal{G} \hat{\xi}$ and $\pi^{\Gamma}(\hat{\xi})=\bigoplus_{i} \pi^{\Gamma}\left(\xi_{i}\right)$.

Proof. Given $\xi=(P, \delta, t) \in \Xi$, let $t=u^{P} c^{P} \in T_{u}^{P} \times T_{r s}^{P}$ be the polar decomposition of $t$ and let $u_{P} c_{P} \in T_{P, u n} \times T_{P, r s}$ be an $\mathcal{A}_{P}$-weight of $\delta$. Put

$$
W_{P, u_{P}}^{+}=\left\{w \in W\left(R_{P}\right): w\left(u_{P}\right)=u_{P}, w\left(R_{P, u_{P}}^{+}\right)=R_{P, u_{P}}^{+}\right\} .
$$

By Theorem 2.1.2 there exists a unique discrete series representation

$$
\delta_{1} \text { of } \mathcal{H}\left(\mathcal{R}_{P, u_{P}}, q_{P, u_{P}}\right) \rtimes W_{P, u_{P}}^{+} \text {such that } \delta \cong \operatorname{Ind}_{\mathcal{H}\left(\mathcal{R}_{P, u_{P}}, q_{P, u_{P}}\right) \rtimes W_{P, u_{P}}^{+}}^{\mathcal{H}_{P}}\left(\delta_{1}\right) \text {. }
$$

Then automatically

$$
\delta \circ \phi_{t} \cong \operatorname{Ind}_{\mathcal{H}\left(\mathcal{R}_{u_{P}}^{P}, q_{u_{P}}^{P}\right) \rtimes W_{P, u_{P}}^{+}}^{\mathcal{H}^{P}}\left(\delta_{1} \circ \phi_{t}\right) .
$$

Let $\delta^{\prime}$ be an irreducible direct summand of the restriction of $\delta_{1}$ to $\mathcal{H}\left(\mathcal{R}_{P, u_{P}}, q_{P, u_{P}}\right)$, such that $u_{P} c_{P}$ is a weight of $\delta^{\prime}$. Then $\delta_{1} \circ \phi_{t}$ is a direct summand of

$$
\operatorname{Ind}_{\mathcal{H}\left(\mathcal{R}_{u_{P}}^{P}, q_{u_{P}}^{P}\right)}^{\mathcal{H}\left(\mathcal{R}_{u_{P}}^{P}, q_{P}^{P}\right) \rtimes W_{P, u_{P}}^{+}}\left(\delta^{\prime} \circ \phi_{t}\right),
$$

so $\pi^{\Gamma}(\xi)$ is a direct summand of

$$
\operatorname{Ind}_{\mathcal{H}\left(\mathcal{R}_{u_{P}}^{P}, q_{u_{P}}^{P}\right)}^{\mathcal{H} \rtimes \Gamma}\left(\delta^{\prime} \circ \phi_{t}\right) .
$$

By Theorem 2.1.4 $\delta^{\prime}$ can also be regarded as a discrete series representation $\tilde{\delta}$ of $\mathbb{H}\left(\tilde{\mathcal{R}}_{P, u_{P}}, k_{P, u_{P}}\right)$ with central character $W\left(R_{P, u_{P}}\right) c_{P}$. Then $\delta^{\prime} \circ \phi_{t}$ corresponds to the representation $\tilde{\delta}_{\log \left(c^{P}\right)}$ of $\mathbb{H}\left(\tilde{\mathcal{R}}_{u_{P}}^{P}, k_{u_{P}}^{P}\right)$. Let $\tilde{P}$ be the unique basis of $R_{P, u_{P}}$ contained in $R_{0}^{+}$.

All in all $(P, \delta, t)$ gives rise to the induction datum $\tilde{\xi}=\left(\tilde{P}, \tilde{\delta}, \log \left(c^{P}\right)\right)$ for the graded Hecke algebra $\mathbb{H}\left(\tilde{\mathcal{R}}_{P, u_{P}}, k_{P, u_{P}}\right)$. Since $R_{P, u_{P}}$ is a parabolic root subsystem of $R_{u}, \tilde{\xi}$ can also be regarded as an induction datum for $\mathbb{H}\left(\tilde{\mathcal{R}}_{u}, k_{u}\right)$. Let us check the possible freedom in the above construction. All $\mathcal{A}_{P}$-weights of $\delta$ are in the same $W_{P}$-orbit, so another choice of $u_{P} c_{P}$ differs only by an element of $W_{P}$. All possible choices of $\delta^{\prime}$ above are conjugated by the action of the group $W_{P, u_{P}}^{+}$, and $\left(W_{0} \rtimes \Gamma\right) u$ is the unitary part of the central character of $\pi^{\Gamma}(\xi)$. Therefore $\xi$ determines the quadruple

$$
\hat{\xi}:=\left(u, \tilde{P}, \tilde{\delta}, \log \left(c^{P}\right)\right) \in \widehat{\Xi}
$$

uniquely modulo the action of $\mathcal{G}$. That yields a map $\Xi \rightarrow \widehat{\Xi} / \mathcal{G}, \xi \rightarrow \mathcal{G} \hat{\xi}$, and since the actions of $\mathcal{G}$ are defined in the same way on both sides, this map factors via $\Xi / \mathcal{G}$.

By reversing the above steps one can reconstruct the representations $\delta^{\prime} \circ \phi_{t}$ and (3.23) from $\tilde{\xi}, u_{P}$ and $u^{P}$. In fact, it suffices to know $\tilde{\xi}$ and the product $u=u_{P} u^{P} \in T_{u n}$. Namely, the only additional ambiguity comes from the group $K_{P}$, but this is inessential since

$$
\left(\delta^{\prime} \circ \psi_{k^{-1}}\right) \circ \phi_{k t} \cong \delta^{\prime} \circ \phi_{t} \text { for } k \in K_{P} .
$$


By construction $\delta_{1}$ is a direct summand of $\operatorname{Ind}_{\mathcal{H}\left(\mathcal{R}_{P, u_{P}}, q_{P, u_{P}}\right)}^{\mathcal{H}\left(\mathcal{R}_{P, u_{P}}, q_{P, u_{P}}\right) \rtimes W_{P, u_{P}}^{+}}\left(\delta^{\prime}\right)$, and the other constituents $\delta_{j}(1<j \leq n)$ are also discrete series representations. Hence (3.23) is a direct sum of finitely many parabolically induced representations

$$
\pi^{\Gamma}\left(P, \operatorname{Ind}_{\mathcal{H}\left(\mathcal{R}_{P, u_{P}}, q_{P, u_{P}}\right) \rtimes W_{P, u_{P}}^{+}}^{\mathcal{H}_{P}}\left(\delta_{j}\right), t\right) .
$$

Now Corollary 2.1.5 assures that our map $\Xi / \mathcal{G} \rightarrow \widehat{\Xi} / \mathcal{G}$ is surjective and that the preimage of $\mathcal{G}\left(u, \tilde{P}, \tilde{\delta}, \log \left(c^{P}\right)\right)$ consists precisely of the association classes

$$
\mathcal{G}\left(P, \operatorname{Ind}_{\mathcal{H}\left(\mathcal{R}_{P, u_{P}}, q_{P, u_{P}}\right) \rtimes W_{P, u_{P}}^{+}}^{\mathcal{H}}\left(\delta_{j}\right), t\right) \quad(1 \leq j \leq n) .
$$

Remark. Things simplify considerably if the group $W_{P, u_{P}}^{+}=\{$id $\}$in (3.21), then the map $\Xi / \mathcal{G} \rightarrow \widehat{\Xi} / \mathcal{G}$ is bijective on $\left(P, \delta, T^{P}\right) / \mathcal{G}$. In many cases this group is indeed trivial, but not always. See OpSo2, Section 8], where $W_{P, u_{P}}^{+}$is denoted $\Gamma_{s(e)}$.

3.4. The geometry of the dual space. For any algebra $A$ the set $\operatorname{Irr}(A)$ has a natural topology, the Jacobson topology. This is the noncommutative generalization of the Zariski topology, by definition all its closed sets are of the form

$$
V(S):=\{\pi \in \operatorname{Irr}(A): \pi(s)=0 \forall s \in S\} \quad S \subset A .
$$

In this section we discuss the topology and the geometry of $\operatorname{Irr}(\mathcal{H} \rtimes \Gamma)$, and we compare it with the dual of $\mathcal{S} \rtimes \Gamma$. This will be useful for the proof of Theorem 2.3.1 and for our discussion of periodic cyclic homology in Section 5.2

Parabolic induction gives, for every discrete series representation $\delta$ of a parabolic subalgebra $\mathcal{H}_{P}$, a family of $\mathcal{H} \rtimes \Gamma$-representations $\pi^{\Gamma}(P, \delta, t)$, parametrized by $t \in$ $T^{P}$. The group

$$
\mathcal{G}_{P, \delta}:=\left\{g \in \mathcal{G}: g(P)=P, \delta \circ \psi_{g}^{-1} \cong \delta\right\}
$$

acts algebraically on $T^{P}$, and by Lemma 3.1.7 points in the same orbit lead to representations with the same irreducible subquotients.

Theorem 3.3 .2 allows us to associate to every $\pi \in \operatorname{Irr}(\mathcal{H} \rtimes \Gamma)$ an induction datum $\xi^{+}(\pi) \in \Xi^{+}$, unique modulo $\mathcal{G}$, such that $\pi$ is a quotient of $\pi^{\Gamma}\left(\xi^{+}(\pi)\right)$. For any subset $U \subset T^{P}$ we define

$$
\operatorname{Irr}_{P, \delta, U}(\mathcal{H} \rtimes \Gamma)=\left\{\pi \in \operatorname{Irr}(\mathcal{H} \rtimes \Gamma): \mathcal{G} \xi^{+}(\pi) \cap(P, \delta, U) \neq \emptyset\right\} .
$$

For $U=T^{P}$ or $U=\{t\}$ we abbreviate this to $\operatorname{Irr}_{P, \delta}(\mathcal{H} \rtimes \Gamma)$ or $\operatorname{Irr}_{P, \delta, t}(\mathcal{H} \rtimes \Gamma)$.

Proposition 3.4.1. Let $U$ be a subset of $T^{P+} T_{u n}^{P}$ such that every $g \in \mathcal{G}_{P, \delta}$ with $g U \cap U \neq \emptyset$ fixes $U$ pointwise. For arbitrary $t \in U$ there are canonical bijections

$$
\operatorname{Irr}_{P, \delta}\left(\mathcal{H}_{P} \rtimes \Gamma_{P, \delta, t}\right) \times U \rightarrow \operatorname{Irr}_{P, \delta, U}\left(\mathcal{H}^{P} \rtimes \Gamma_{P, \delta, t}\right) \rightarrow \operatorname{Irr}_{P, \delta, U}(\mathcal{H} \rtimes \Gamma) .
$$

Remark. It is not unreasonable to expect that the Jacobson topology of $\mathcal{H} \rtimes \Gamma$ induces the Zariski topology on $\operatorname{Irr}_{P, \delta}\left(\mathcal{H}_{P} \rtimes \Gamma_{P, \delta, t}\right) \times U$, where $\operatorname{Irr}_{P, \delta}\left(\mathcal{H}_{P} \rtimes \Gamma_{P, \delta, t}\right)$ is regarded as a discrete space. However, while it is easy to see that all $V(h)$ become Zariski-closed in $\operatorname{Irr}_{P, \delta}\left(\mathcal{H}_{P} \rtimes \Gamma_{P, \delta, t}\right) \times U$, it is not clear that one can obtain all Zariski-closed subsets in this way. That might require some extra conditions on $U$.

Proof. By assumption every $t \in U$ has the same stabilizer $\mathcal{G}_{P, \delta, t} \subset \mathcal{G}_{P, \delta}$. According to Theorem 3.3.1 the operators $\left\{\pi^{\Gamma}(g, P, \delta, t): g \in \mathcal{G}_{P, \delta, t}\right\} \operatorname{span} \operatorname{End}_{\mathcal{H} \rtimes \Gamma}\left(\pi^{\Gamma}(P, \delta, t)\right)$. By definition all elements of $\operatorname{Irr}_{P, \delta, t}(\mathcal{H} \rtimes \Gamma)$ occur as a quotient of $\pi^{\Gamma}(P, \delta, t)$, but the latter representation also has other constituents if it is not completely reducible. We have to avoid that situation if we want to find a direct relation between $\operatorname{End}_{\mathcal{H} \rtimes \Gamma}\left(\pi^{\Gamma}(P, \delta, t)\right)$ and $\operatorname{Irr}_{P, \delta, t}(\mathcal{H} \rtimes \Gamma)$. 
Since $\pi^{\Gamma}(P, \delta, t)$ and $\pi^{\Gamma}(g, P, \delta, t)$ are unitary for $t \in T_{u n}^{P}$, there exists an open $\mathcal{G}_{P, \delta}$-stable tubular neighborhood $T_{\epsilon}^{P}$ of $T_{u n}^{P}$ in $T^{P}$, such that $\pi^{\Gamma}(P, \delta, t)$ is completely reducible and $\pi^{\Gamma}(g, P, \delta, t)$ is regular and invertible, for all $t \in T_{\epsilon}^{P}$ and $g \in \mathcal{G}_{P, \delta}$. For every $t \in U$ we can find $r \in \mathbb{R}_{>0}$ such that $t|t|^{r-1} \in T_{\epsilon}^{P}$. Let $U_{\epsilon} \subset T_{\epsilon}^{P}$ be the resulting collection. For every $t \in U_{\epsilon}$ the algebras

$$
\left\{\pi^{\Gamma}(P, \delta, t)(h): h \in \mathcal{H} \rtimes \Gamma\right\} \text { and } \operatorname{span}\left\{\pi^{\Gamma}(g, P, \delta, t): g \in \mathcal{G}_{P, \delta, t}\right\}
$$

are each others' commutant in

$$
\operatorname{End}_{\mathbb{C}}\left(\pi^{\Gamma}(P, \delta, t)\right)=\operatorname{End}_{\mathbb{C}}\left(\mathbb{C}\left[\Gamma W^{P}\right] \otimes_{\mathbb{C}} V_{\delta}\right) .
$$

Hence there is a natural bijection between

- isotypical components of $\pi^{\Gamma}(P, \delta, t)$ as a $\mathcal{H} \rtimes \Gamma$-representation;

- isotypical components of $\pi^{\Gamma}(P, \delta, t)$ as a $\mathcal{G}_{P, \delta, t}$-representation.

The operators $\pi^{\Gamma}(P, \delta, t)$ are rational in $t \in T^{P}$, and regular on $T_{\epsilon}^{P}$. As there are only finitely many inequivalent $\mathcal{G}_{P, \delta, t}$-representations of a fixed finite dimension, the isomorphism class of $\pi^{\Gamma}(P, \delta, t)$ as a $\mathcal{G}_{P, \delta, t}$-representation does not depend on $t \in U_{\epsilon}$. This provides a natural bijection

$$
\operatorname{Irr}_{P, \delta, U_{\epsilon}}(\mathcal{H} \rtimes \Gamma) \longleftrightarrow \operatorname{Irr}_{P, \delta, t}(\mathcal{H} \rtimes \Gamma) \times U_{\epsilon} \quad t \in U_{\epsilon} .
$$

The extended Langlands classification (Corollary 2.2.5) shows that there is a canonical bijection $\operatorname{Irr}_{P, \delta, t|t|^{r-1}}(\mathcal{H} \rtimes \Gamma) \leftrightarrow \operatorname{Irr}_{P, \delta, t}(\mathcal{H} \rtimes \Gamma)$ for every $r \in \mathbb{R}_{>0}$, which allows us to extend (3.24) uniquely to

$$
\operatorname{Irr}_{P, \delta, U}(\mathcal{H} \rtimes \Gamma) \longleftrightarrow \operatorname{Irr}_{P, \delta, t_{0}}(\mathcal{H} \rtimes \Gamma) \times U \quad t_{0} \in U .
$$

The above also holds with the algebras $\mathcal{H} \rtimes \Gamma_{P, \delta, t}$ or $\mathcal{H}_{P} \rtimes \Gamma_{P, \delta, t}$ in the role of $\mathcal{H} \rtimes \Gamma$. Since the construction of the intertwiners corresponding to $g \in \mathcal{G}_{P, \delta, t}$ is the same in all three cases, we obtain natural isomorphisms

$$
\begin{aligned}
\operatorname{End}_{\mathcal{H}_{P} \rtimes \Gamma_{P, \delta, t}}\left(\operatorname{Ind}_{\mathcal{H}_{P}}^{\mathcal{H}_{P} \rtimes \Gamma_{P, \delta, t}} \delta\right) & \cong \operatorname{End}_{\mathcal{H}^{P} \rtimes \Gamma_{P, \delta, t}}\left(\pi^{P, \Gamma_{P, \delta, t}}(P, \delta, t)\right) \\
& \cong \operatorname{End}_{\mathcal{H} \rtimes \Gamma}\left(\pi^{\Gamma}(P, \delta, t)\right) .
\end{aligned}
$$

Now the above bijection between isotypical components shows that the maps

$$
\begin{aligned}
\operatorname{Irr}_{P, \delta}\left(\mathcal{H}_{P} \rtimes \Gamma_{P, \delta, t}\right) & \rightarrow \operatorname{Irr}_{P, \delta, t}\left(\mathcal{H}^{P} \rtimes \Gamma_{P, \delta, t}\right) \rightarrow \operatorname{Irr}_{P, \delta, t}(\mathcal{H} \rtimes \Gamma), \\
\rho & \mapsto \rho \circ \phi_{t} \mapsto \operatorname{Ind}_{\mathcal{H}^{P} \rtimes \Gamma_{P, \delta, t}}^{\mathcal{H} \Gamma}\left(\rho \circ \phi_{t}\right)
\end{aligned}
$$

are bijective, and (3.25) allows us to extend this from one $t$ to $U$.

Theorem 3.3 .2 shows that $\operatorname{Irr}_{P, \delta}(\mathcal{H} \rtimes \Gamma)$ and $\operatorname{Irr}_{Q, \sigma}(\mathcal{H} \rtimes \Gamma)$ are either equal or disjoint, depending on whether or not $(P, \delta)$ and $(Q, \sigma)$ are $\mathcal{G}$-associate. The sets $\operatorname{Irr}_{P, \delta}(\mathcal{H} \rtimes \Gamma)$ are usually not closed in $\operatorname{Irr}(\mathcal{H} \rtimes \Gamma)$, because we did not include all constituents of the representations $\pi^{\Gamma}(P, \delta, t)$. We can use their closures to define a stratification of $\operatorname{Irr}(\mathcal{H} \rtimes \Gamma)$, and a corresponding stratification of $\operatorname{Irr}(\mathcal{S} \rtimes \Gamma)$. By Corollary 3.1 .3 we may identify $\operatorname{Irr}_{P, \delta}(\mathcal{S} \rtimes \Gamma)$ with the tempered part $\operatorname{Irr}_{P, \delta, T_{u n}^{P}}(\mathcal{H} \rtimes$ $\Gamma)$ of $\operatorname{Irr}_{P, \delta}(\mathcal{H} \rtimes \Gamma)$.

Let $\Delta$ be a set of representatives for the action of $\mathcal{G}$ on pairs $(P, \delta)$. Then the cardinality of $\Delta$ equals the number of connected components of $\Xi_{u n} / \mathcal{G}$ and by Theorem 3.2 .2

$$
\mathcal{S} \rtimes \Gamma \cong \bigoplus_{(P, \delta) \in \Delta}\left(C^{\infty}\left(T_{u n}^{P}\right) \otimes_{\mathbb{C}} \operatorname{End}\left(\mathbb{C}\left[\Gamma W^{P}\right] \otimes_{\mathbb{C}} V_{\delta}\right)\right)^{\mathcal{G}_{P, \delta}} .
$$


Lemma 3.4.2. There exist filtrations by two-sided ideals

$$
\begin{array}{ccccccc}
\mathcal{H} \rtimes \Gamma=F_{0}(\mathcal{H} \rtimes \Gamma) & \supset & F_{1}(\mathcal{H} \rtimes \Gamma) & \supset & \cdots & \supset & F_{|\Delta|}(\mathcal{H} \rtimes \Gamma)=0, \\
\mathcal{S} \rtimes \Gamma=F_{0}(\mathcal{S} \rtimes \Gamma) & \supset & F_{1}(\mathcal{S} \rtimes \Gamma) & \supset & \cdots & \supset & F_{|\Delta|}(\mathcal{S} \rtimes \Gamma)=0,
\end{array}
$$

with $F_{i}(\mathcal{H} \rtimes \Gamma) \subset F_{i}(\mathcal{S} \rtimes \Gamma)$, such that for all $i>0$ :

(a) $\operatorname{Irr}\left(F_{i-1}(\mathcal{S} \rtimes \Gamma) / F_{i}(\mathcal{S} \rtimes \Gamma)\right) \cong \operatorname{Irr}_{P_{i}, \delta_{i}}(\mathcal{S} \rtimes \Gamma)$,

(b) $\operatorname{Irr}\left(F_{i-1}(\mathcal{H} \rtimes \Gamma) / F_{i}(\mathcal{H} \rtimes \Gamma)\right) \cong \operatorname{Irr}_{P_{i}, \delta_{i}}(\mathcal{H} \rtimes \Gamma)$.

Remark. Analogous filtrations of Hecke algebras of reductive $p$-adic groups are described in [Sol4, Lemma 2.17]. The proof in our setting is basically the same.

Proof. We number the elements of $\Delta$ such that

$$
\left\|c c_{P_{i}}\left(\delta_{i}\right)\right\| \geq\left\|c c_{P_{j}}\left(\delta_{j}\right)\right\| \quad \text { if } j \leq i
$$

and we define

$$
\begin{aligned}
F_{i}(\mathcal{H} \rtimes \Gamma) & =\left\{h \in \mathcal{H} \rtimes \Gamma: \pi(h)=0 \text { for all } \pi \in \operatorname{Irr}_{P_{j}, \delta_{j}}(\mathcal{H} \rtimes \Gamma), j \leq i\right\}, \\
F_{i}(\mathcal{S} \rtimes \Gamma) & =\left\{h \in \mathcal{S} \rtimes \Gamma: \pi(h)=0 \text { for all } \pi \in \operatorname{Irr}_{P_{j}, \delta_{j}}(\mathcal{S} \rtimes \Gamma), j \leq i\right\} .
\end{aligned}
$$

Clearly, $F_{i}(\mathcal{H} \rtimes \Gamma) \subset F_{i}(\mathcal{S} \rtimes \Gamma)$ and

$$
F_{i-1}(\mathcal{S} \rtimes \Gamma) / F_{i}(\mathcal{S} \rtimes \Gamma) \cong\left(C^{\infty}\left(T_{u n}^{P}\right) \otimes_{\mathbb{C}} \operatorname{End}\left(\mathbb{C}\left[\Gamma \times W^{P}\right] \otimes_{\mathbb{C}} V_{\delta}\right)\right)^{\mathcal{G}_{P_{i}, \delta_{i}}},
$$

which establishes (a). For (b), we first show that

$$
\bigcup_{j \leq i} \operatorname{Irr}_{P_{j}, \delta_{j}}(\mathcal{H} \rtimes \Gamma)
$$

is closed in the Jacobson topology of $\operatorname{Irr}(\mathcal{H} \rtimes \Gamma)$. Its Jacobson-closure consists of all irreducible subquotients $\pi$ of $\pi^{\Gamma}\left(P_{j}, \delta_{j}, t\right)$, for $j \leq i$ and $t \in T^{P_{j}}$. Suppose that $\pi \notin \operatorname{Irr}_{P_{j}, \delta_{j}}(\mathcal{H} \rtimes \Gamma)$. By Theorem 3.3.2 $\pi \in \operatorname{Irr}_{P, \delta}(\mathcal{H} \rtimes \Gamma)$ for some discrete series representation $\delta$ of $\mathcal{H}_{P}$ with $\left\|c c_{P}(\delta)\right\|>\left\|c c_{P_{j}}\left(\delta_{j}\right)\right\|$. Select $\left(P_{n}, \delta_{n}\right)$ and $g \in \mathcal{G}$ such that $g(P, \delta)=\left(P_{n}, \delta_{n}\right)$. Then $\pi \in \operatorname{Irr}_{P_{n}, \delta_{n}}(\mathcal{H} \rtimes \Gamma)$ and

$$
\left\|c c_{P_{n}}\left(\delta_{n}\right)\right\|=\left\|c c_{P}(\delta)\right\|>\left\|c c_{P_{j}}\left(\delta_{j}\right)\right\|,
$$

so $n<j \leq i$ by (3.27). Therefore (3.28) is indeed Jacobson-closed and

$$
\operatorname{Irr}\left(F_{i}(\mathcal{H} \rtimes \Gamma)\right) \cong \bigcup_{j<i} \operatorname{Irr}_{P_{j}, \delta_{j}}(\mathcal{H} \rtimes \Gamma),
$$

which implies (b).

The filtrations from Lemma 3.4 .2 help us to compare the dual of $\mathcal{H} \rtimes \Gamma$ with its tempered dual, which can be identified with the dual of $\mathcal{S} \rtimes \Gamma$. The space $\operatorname{Irr}_{P, \delta}(\mathcal{H} \rtimes \Gamma)$ comes with a finite-to-one projection onto

$$
T^{P+} T_{u n}^{P} / \mathcal{G}_{P, \delta} \cong T^{P} /\left(W\left(R_{P}\right) \rtimes \mathcal{G}_{P, \delta}\right) .
$$

The subspace $\operatorname{Irr}_{P, \delta}(\mathcal{S} \rtimes \Gamma) \subset \operatorname{Irr}_{P, \delta}(\mathcal{H} \rtimes \Gamma)$ is the inverse image of $T_{u n}^{P} /\left(W\left(R_{P}\right) \rtimes\right.$ $\left.\mathcal{G}_{P, \delta}\right)$ under this projection. By Proposition 3.4.1 the fiber at $t \in T^{P}$ essentially depends only on the stabilizer $\mathcal{G}_{P, \delta, t}$. Since $\mathcal{G}_{P, \delta}$ acts algebraically on $T^{P}$, the collection of points $t \in T^{P}$ such that the fiber at $\left(W_{P} \rtimes \mathcal{G}_{P, \delta}\right) t$ has exactly $m$ points (for some fixed $m \in \mathbb{N}$ ) is a complex affine variety, say $T^{P, m}$. As the action of $\mathcal{G}_{P, \delta}$ stabilizes $T_{u n}^{P}$, the variety $T^{P, m}$ is already determined by its intersection with $T_{u n}^{P}$. Hence one can reconstruct the set $\operatorname{Irr}_{P, \delta}(\mathcal{H} \rtimes \Gamma)$ from $\operatorname{Irr}_{P, \delta}(\mathcal{S} \rtimes \Gamma)$. 
To complete the proof of Theorem 2.3.1 a we will introduce some terminology. We know from Lemma 3.1.1 that representations of the form

$$
\pi^{P, \Gamma_{P, \delta, u}}(P, \delta, u)=\operatorname{Ind}_{\mathcal{H}^{P}}^{\mathcal{H}^{P} \rtimes \Gamma_{P, \delta, u}}\left(\delta \circ \phi_{u}\right) \quad \text { with }(P, \delta, u) \in \Xi_{u n}
$$

are tempered and unitary. Let $\sigma$ be an irreducible summand of such an $\mathcal{H}^{P} \rtimes$ $\Gamma_{P, \delta, u}$-representation and let $T^{\sigma}$ be the connected component of $T^{W\left(R_{P}\right) \rtimes \Gamma_{P, \delta, u}}$ that contains $1 \in T$. Notice that $T^{\sigma} \subset T^{P}$ because $T_{P}^{W\left(R_{P}\right)}$ is finite. Following Opd3 we call

$$
\left\{\operatorname{Ind}_{\mathcal{H}^{P} \rtimes \Gamma_{P, \delta, u}}^{\mathcal{H} \rtimes}\left(\sigma \circ \phi_{t}\right): t \in T^{\sigma}\right\}
$$

a smooth $d$-dimensional family of representations, where $d$ is the dimension of

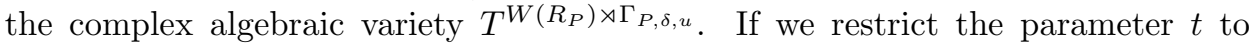
$T_{u n}^{\sigma}=T^{\sigma} \cap T_{u n}$, then we add the adjective tempered to this description. Since there are only finitely many pairs $(P, \delta)$ and since two $u$ 's with the same $u T^{\sigma}$ give rise to the same smooth family, there exist only finitely many smooth families of $\mathcal{H} \rtimes \Gamma$-representations.

For $t \in T^{\sigma}$ such that $\mathcal{G}_{P, \delta, u t}=\mathcal{G}_{P, \delta, u}$ and all the intertwiners $\pi^{\Gamma}(g, P, \delta, u t)$ with $g \in \mathcal{G}_{P, \delta, u}$ are invertible, the representation $\operatorname{Ind}_{\mathcal{H}^{P} \rtimes \Gamma_{P, \delta, u}}^{\mathcal{H} \rtimes \Gamma}\left(\sigma \circ \phi_{t}\right)$ is irreducible by Proposition 3.4.1. Hence the family (3.30) consists of representations which are irreducible on a Zariski-open dense subset of the parameter space $T^{\sigma}$.

The proof of Proposition 3.1.4 b shows that

$$
\operatorname{Ind}_{\mathcal{H}^{P} \rtimes \Gamma_{P, \delta, u}}^{\mathcal{H} \rtimes \Gamma}\left(\sigma \circ \phi_{t}\right)
$$

is always a direct sum of representations of the form $\pi^{\Gamma}\left(P^{\prime}, \sigma^{\prime}, t^{\prime}, \rho^{\prime}\right)$, where $\left(P^{\prime}, \sigma^{\prime}, t^{\prime}, \rho^{\prime}\right)$ is almost a Langlands datum for $\mathcal{H} \rtimes \Gamma$, the only difference being that $\left|t^{\prime}\right| \in T_{r s}^{P^{\prime}}$ need not be positive. Nevertheless, we can specify a "Langlands constituent" of $\pi^{\Gamma}\left(P^{\prime}, \sigma^{\prime}, t^{\prime}, \rho^{\prime}\right)$ :

Lemma 3.4.3. Let $Q \subset F_{0}, t \in T^{Q}$, let $\tau$ be an irreducible tempered $\mathcal{H}_{Q}$-representation and let $\rho$ be an irreducible representation of $\mathbb{C}\left[\Gamma_{Q, \tau, t}, \kappa\right]$. The representation $\pi^{\Gamma}(Q, \tau, t, \rho)$ defined by (2.15) has a unique "Langlands" constituent $L\left(\pi^{\Gamma}(Q, \tau, t, \rho)\right)$ which is minimal in the sense of Lemma 2.2.6. b. Moreover, $L\left(\pi^{\Gamma}(Q, \tau, t, \rho)\right)$ occurs with multiplicity one in $\pi^{\Gamma}(Q, \tau, t, \rho)$.

Proof. Choose $g \in \mathcal{G}$ such that $g(Q, \tau, t) \in \Xi^{+}$. By Proposition 3.1.4.b $g(Q, \tau, t, \rho)$ is a Langlands datum for $\mathcal{H} \rtimes \Gamma$ and by Theorem 3.3.1 it does not matter which $g \in \mathcal{G}$ with this property we take. Like in Lemma 3.1.7 $\pi^{\Gamma}(g(Q, \tau, t, \rho))$ has the same trace and the same semisimplication as $\pi^{\Gamma}(Q, \tau, t, \rho)$. Using Corollary 2.2.5 a we define

$$
L\left(\pi^{\Gamma}(Q, \tau, t, \rho)\right):=L^{\Gamma}(g(Q, \tau, t, \rho)) .
$$

Lemma 2.2.6 b characterizes this as the unique constituent which is minimal in the appropriate sense, and it shows that $L\left(\pi^{\Gamma}(Q, \tau, t, \rho)\right)$ appears only once in $\pi^{\Gamma}(Q, \tau, t, \rho)$.

Let $L\left(\operatorname{Ind}_{\mathcal{H}^{P} \rtimes \Gamma_{P, \delta, u}}^{\mathcal{H} \rtimes \Gamma}\left(\sigma \circ \phi_{t}\right)\right)$ be the direct sum of the Langlands constituents of the irreducible summands of $\operatorname{Ind}_{\mathcal{H}^{P} \rtimes \Gamma_{P, \delta, u}}^{\mathcal{H} \rtimes \Gamma}\left(\sigma \circ \phi_{t}\right)$. The family

$$
\left\{L\left(\operatorname{Ind}_{\mathcal{H}^{P} \rtimes \Gamma_{P, \delta, u}}^{\mathcal{H} \rtimes \Gamma}\left(\sigma \circ \phi_{t}\right)\right): t \in T^{\sigma}\right\}
$$


cannot be called smooth, because the traces of these representations do not depend continuously on $t$. Let us call it an L-smooth $d$-dimensional family of representations.

Continuation of the proof of Theorem 2.3.1. We have to show that the $\mathbb{Q}$-linear extension

$$
\zeta_{\mathbb{Q}}^{*}: G_{\mathbb{Q}}(\mathcal{H} \rtimes \Gamma) \rightarrow G_{\mathbb{Q}}\left(X \rtimes\left(W_{0} \rtimes \Gamma\right)\right) .
$$

of $\zeta^{*}$ is a bijection. By properties (d) and (e) of Theorem 2.3.1

$$
\zeta_{\mathbb{Q}}^{*} L\left(\operatorname{Ind}_{\mathcal{H}^{P} \rtimes \Gamma_{P, \delta, u}}^{\mathcal{H} \rtimes \Gamma}\left(\sigma \circ \phi_{t}\right)\right)=\operatorname{Ind}_{X \rtimes\left(W\left(R_{P}\right) \rtimes \Gamma_{P, \delta, u}\right)}^{X \rtimes\left(W_{0} \rtimes \Gamma\right)}\left(\zeta_{\mathbb{Q}}^{*}(\sigma) \circ \phi_{t}\right) .
$$

The right-hand side is almost a smooth $d$-dimensional family of $X \rtimes\left(W_{0} \rtimes \Gamma\right)$ representations. Not entirely, because $\zeta_{\mathbb{Q}}^{*}(\sigma)$ is in general reducible and because a priori this family could be only a part of a higher dimensional smooth family.

Let $G_{\mathbb{Q}}^{d}(\mathcal{H} \rtimes \Gamma)$ be the $\mathbb{Q}$-submodule of $G_{\mathbb{Q}}(\mathcal{H} \rtimes \Gamma)$ spanned by the representations (3.32), for all L-smooth families of dimension at least $d \in \mathbb{Z}_{\geq 0}$. This is a decreasing sequence of $\mathbb{Q}$-submodules of $G_{\mathbb{Q}}(\mathcal{H} \rtimes \Gamma)$, by convention

$$
G_{\mathbb{Q}}^{0}(\mathcal{H} \rtimes \Gamma)=G_{\mathbb{Q}}(\mathcal{H} \rtimes \Gamma)
$$

and $G_{\mathbb{Q}}^{d}(\mathcal{H} \rtimes \Gamma)=0$ when $d>\operatorname{dim}_{\mathbb{C}}(T)=\operatorname{rank}(X)$. We define $G_{\mathbb{Q}}^{d}(\mathcal{S} \rtimes \Gamma)$ analogously, with tempered smooth families of dimension at least $d$. Now (3.34) says that

$$
\begin{array}{lll}
\zeta_{\mathbb{Q}}^{*}\left(G_{\mathbb{Q}}^{d}(\mathcal{H} \rtimes \Gamma)\right) & \subset G_{\mathbb{Q}}^{d}\left(X \rtimes W_{0} \rtimes \Gamma\right), \\
\zeta_{\mathbb{Q}}^{*}\left(G_{\mathbb{Q}}^{d}(\mathcal{S} \rtimes \Gamma)\right) & \subset \quad G_{\mathbb{Q}}^{d}\left(\mathcal{S}(X) \rtimes W_{0} \rtimes \Gamma\right) .
\end{array}
$$

Let us consider the graded vector spaces associated to these filtrations. For tempered representations $\zeta_{\mathbb{Q}}^{*}$ induces $\mathbb{Q}$-linear maps

$$
G_{\mathbb{Q}}^{d}(\mathcal{S} \rtimes \Gamma) / G_{\mathbb{Q}}^{d+1}(\mathcal{S} \rtimes \Gamma) \rightarrow G_{\mathbb{Q}}^{d}(\mathcal{S}(W) \rtimes \Gamma) / G_{\mathbb{Q}}^{d+1}(\mathcal{S}(W) \rtimes \Gamma) .
$$

We proved in Section 2.3 that

$$
\zeta_{\mathbb{Q}}^{*}: G_{\mathbb{Q}}(\mathcal{S} \rtimes \Gamma) \rightarrow G_{\mathbb{Q}}(\mathcal{S}(W) \rtimes \Gamma)
$$

is bijective. Since there are only finitely many smooth families, it is impossible to fill up a $d$-dimensional tempered smooth family with smooth families of dimension smaller than $d$. Therefore (3.36) is bijective for all $d \in \mathbb{Z}_{\geq 0}$. We will show that

$$
G_{\mathbb{Q}}^{d}(\mathcal{H} \rtimes \Gamma) / G_{\mathbb{Q}}^{d+1}(\mathcal{H} \rtimes \Gamma) \rightarrow G_{\mathbb{Q}}^{d}\left(X \rtimes W_{0} \rtimes \Gamma\right) / G_{\mathbb{Q}}^{d+1}\left(X \rtimes W_{0} \rtimes \Gamma\right)
$$

is bijective as well. For $d>\operatorname{dim}_{\mathbb{C}}(T)$ there is nothing to prove, so take $d \leq \operatorname{dim}_{\mathbb{C}}(T)$. Pick smooth $d$-dimensional families $\left\{\pi_{i, t}: i \in I, t \in V_{i}\right\}$ such that

$$
\left\{\pi_{i, t}: i \in I, t \in\left(V_{i} \cap T_{u n}\right) / \sim\right\}
$$

is a basis of $G_{\mathbb{Q}}^{d}(\mathcal{S} \rtimes \Gamma) / G_{\mathbb{Q}}^{d+1}(\mathcal{S} \rtimes \Gamma)$, where $t \sim t^{\prime}$ if and only if $\pi_{i, t} \cong \pi_{i, t^{\prime}}$. The bijectivity of (3.36) implies that

$$
\left\{\zeta_{\mathbb{Q}}^{*}\left(\pi_{i, t}\right): i \in I, t \in\left(V_{i} \cap T_{u n}\right) / \sim\right\}
$$

is a basis of $G_{\mathbb{Q}}^{d}(\mathcal{S}(W) \rtimes \Gamma) / G_{\mathbb{\mathbb { N }}}^{d+1}(\mathcal{S}(W) \rtimes \Gamma)$. The discussion following (3.29) shows that L-smooth $d$-dimensional families and tempered smooth $d$-dimensional families are in natural bijection. Hence

$$
\left\{L\left(\pi_{i, t}\right): i \in I, t \in V_{i} / \sim\right\}
$$


is a basis of $G_{\mathbb{Q}}^{d}(\mathcal{H} \rtimes \Gamma) / G_{\mathbb{Q}}^{d+1}(\mathcal{H} \rtimes \Gamma)$ and

$$
\left\{\zeta_{\mathbb{Q}}^{*}\left(\pi_{i, t}\right): i \in I, t \in V_{i} / \sim\right\}
$$

is a basis of $G_{\mathbb{Q}}^{d}\left(X \rtimes W_{0} \rtimes \Gamma\right) / G_{\mathbb{Q}}^{d+1}\left(X \rtimes W_{0} \rtimes \Gamma\right)$. Therefore (3.38) is indeed bijective. From this we deduce, with some standard applications of the five lemma, that (3.33) is a bijection.

\section{Parameter Deformations}

Let $\mathcal{H}=\mathcal{H}(\mathcal{R}, q)$ be an affine Hecke algebra associated to an equal parameter function $q$. Varying the parameter $q \in \mathbb{C}^{\times}$yields a family of algebras, whose members are specializations of an affine Hecke algebra with a formal variable $\mathbf{q}$. The Kazhdan-Lusztig-parametrization of $\operatorname{Irr}(\mathcal{H}(\mathcal{R}, q)$ ) KaLu2, Ree2 provides a bijection between the irreducible representations of $\mathcal{H}(\mathcal{R}, q)$ and $\mathcal{H}\left(\mathcal{R}, q^{\prime}\right)$, as long as $q, q^{\prime} \in \mathbb{C}^{\times}$are not roots of unity. Moreover, every $\pi_{q} \in \operatorname{Irr}(\mathcal{H}(\mathcal{R}, q))$ is part of a family of representations $\left\{\pi_{q}: q \in \mathbb{C}^{\times}\right\}$which depends algebraically on $q$.

It is our conviction that a similar structure underlies the representation theory of affine Hecke algebras with unequal parameters. However, at present a proof seems to be out of reach. We have more control when we restrict ourselves to positive parameter functions and to parameter deformations of the form $q \mapsto q^{\epsilon}$ with $\epsilon \in \mathbb{R}$. We call this scaling of the parameter function, because it corresponds to multiplying the parameters of a graded Hecke algebra with $\epsilon$. Notice that $\mathcal{H}\left(\mathcal{R}, q^{0}\right)=\mathbb{C}[W]$.

We can relate representations of $\mathcal{H}(\mathcal{R}, q)$ to $\mathcal{H}\left(\mathcal{R}, q^{\epsilon}\right)$-representations by applying a similar scaling operation on suitable subsets of the space $T \cong \operatorname{Irr}(\mathcal{A})$. We construct a family of functors

$$
\tilde{\sigma}_{\epsilon}: \operatorname{Mod}_{f}(\mathcal{H}(\mathcal{R}, q)) \rightarrow \operatorname{Mod}_{f}\left(\mathcal{H}\left(\mathcal{R}, q^{\epsilon}\right)\right)
$$

which is an equivalence of categories for $\epsilon \neq 0$, and which preserves many properties of representations (Corollary 4.2.2). In particular, this provides families of representations $\left\{\tilde{\sigma}_{\epsilon}(\pi): \epsilon \in \mathbb{R}\right\}$ that depend analytically on $\epsilon$.

The Schwartz algebra $\mathcal{S}(\mathcal{R}, q)$ behaves even better under scaling of the parameter function $q$. As $q$ can be varied in several directions, we have a higher dimensional family of Fréchet algebras $\mathcal{S}(\mathcal{R}, q)$, which is known to depend continuously on $q$ OpSo2, Appendix A]. This was exploited for the main results of OpSo2, but the techniques used there to study general deformations of $q$ are specific to discrete series representations.

To get going at the other series, we only scale $q$. Via a detailed study of the Fourier transform of $\mathcal{S}(\mathcal{R}, q)$ (see Theorem 3.2.2) we construct homomorphisms of Fréchet *-algebras

$$
\zeta_{\epsilon}: \mathcal{S}\left(\mathcal{R}, q^{\epsilon}\right) \rightarrow \mathcal{S}(\mathcal{R}, q) \quad \epsilon \in[0,1]
$$

which depend piecewise analytically on $\epsilon$ and are isomorphisms for $\epsilon>0$ (Theorem 4.4.2). It is not known whether this is possible with $\mathcal{H}(\mathcal{R}, q)$ instead of $\mathcal{S}(\mathcal{R}, q)$, when $q$ is not an equal parameter function.

The most remarkable part is that these maps extend continuously to $\epsilon=0$, that is, to a map $\zeta_{0}: \mathcal{S}(W) \rightarrow \mathcal{S}(\mathcal{R}, q)$. Of course $\zeta_{0}$ cannot be an isomorphism, but it is injective and and some ways behaves like an isomorphism. In fact, we show that for irreducible tempered $\mathcal{H}(\mathcal{R}, q)$-representations the map $\zeta^{*}$ from Section 2.3 agrees with the functors $\tilde{\sigma}_{0}$ and $\pi \mapsto \pi \circ \zeta_{0}$ (Corollary 4.4.3). 
4.1. Scaling Hecke algebras. As we saw in Section 2.3, there is a correspondence between tempered representations of $\mathcal{H} \rtimes \Gamma$ and of $W \rtimes \Gamma$. On central characters this correspondence has the effect of forgetting the real split part and retaining the unitary part. These elements of $T /\left(W_{0} \rtimes \Gamma\right)$ are connected by the path $\left(W_{0} \rtimes \Gamma\right) c^{\epsilon} u$, with $\epsilon \in[0,1]$. Opdam Opd2, Section 5] was the first to realize that one can interpolate not only the central characters, but also the representations themselves. In this section we will recall and generalize the relevant results of Opdam. In contrast to the previous sections we will not include an extra diagram automorphism group $\Gamma$ in our considerations, as the notation is already heavy enough. However, it can be checked easily that all the results admit obvious generalizations with such a $\Gamma$.

First we discuss the situation for graded Hecke algebras, which is considerably easier. Let $V \subset \mathfrak{t}$ be a nonempty open $W_{0}$-invariant subset. Recall the elements $\tilde{\imath}_{w} \in C^{m e}(V)^{W_{0}} \otimes_{Z(\mathbb{H}(\tilde{\mathcal{R}}, k))} \mathbb{H}(\tilde{\mathcal{R}}, k)$ from Proposition 1.5.1. Given any $\epsilon \in \mathbb{C}$ we define a scaling map

$$
\lambda_{\epsilon}: V \rightarrow \epsilon V, v \mapsto \epsilon v .
$$

For $\epsilon \neq 0$ it induces an algebra isomorphism

$$
\begin{aligned}
m_{\epsilon}: C^{m e}(\epsilon V)^{W_{0}} \otimes_{Z(\mathbb{H}(\tilde{\mathcal{R}}, \epsilon k))} \mathbb{H}(\tilde{\mathcal{R}}, k) & \rightarrow C^{m e}(V)^{W_{0}} \otimes_{Z(\mathbb{H}(\tilde{\mathcal{R}}, k))} \mathbb{H}(\tilde{\mathcal{R}}, k), \\
f \tilde{\imath}_{w, \epsilon} & \mapsto\left(f \circ \lambda_{\epsilon}\right) \tilde{\imath}_{w} .
\end{aligned}
$$

Let us calculate the image of a simple reflection $s_{\alpha} \in S_{0}$ :

$$
\begin{aligned}
m_{\epsilon}\left(1+s_{\alpha}\right) & =m_{\epsilon}\left(\tilde{c}_{\alpha, \epsilon}^{-1}\left(1+\tilde{\imath}_{s_{\alpha}, \epsilon}\right)\right)=m_{\epsilon}\left(\frac{\alpha}{\epsilon k_{\alpha}+\alpha}\left(1+\tilde{\imath}_{s_{\alpha}, \epsilon}\right)\right)=\frac{\epsilon \alpha}{\epsilon k_{\alpha}+\epsilon \alpha}\left(1+\tilde{\imath}_{s_{\alpha}}\right) \\
& =\tilde{c}_{\alpha}^{-1}\left(1+\tilde{\imath}_{s_{\alpha}}\right)=1+s_{\alpha} .
\end{aligned}
$$

That is, $m_{\epsilon}(w)=w$ for all $w \in W_{0} \subset \mathbb{H}(\tilde{\mathcal{R}}, \epsilon k)$, so $m_{\epsilon}$ is indeed the same as (1.11). In particular, we see that $m_{\epsilon}$ can be defined without using meromorphic functions. These maps have a limit homomorphism

$$
\begin{aligned}
m_{0}: \quad \mathbb{H}(\tilde{\mathcal{R}}, 0)=\mathcal{O}(\mathfrak{t}) \rtimes W_{0} & \rightarrow \mathbb{H}(\tilde{\mathcal{R}}, k), \\
f w & \mapsto f(0) w,
\end{aligned}
$$

with the property that

$$
\mathbb{C} \rightarrow C^{m e}(V)^{W_{0}} \otimes_{Z(\mathbb{H}(\tilde{\mathcal{R}}, k))} \mathbb{H}(\tilde{\mathcal{R}}, k): \epsilon \mapsto m_{\epsilon}(f w)
$$

is analytic for all $f \in \mathcal{O}(\mathfrak{t})$ and $w \in W_{0}$.

From now we assume that $\epsilon$ is real. Let $q^{\epsilon}$ be the parameter function $q^{\epsilon}(w)=$ $q(w)^{\epsilon}$, which is well defined because $q(w) \in \mathbb{R}_{>0}$ for all $w \in W$. We obtain a family of algebras $\mathcal{H}_{\epsilon}=\mathcal{H}\left(\mathcal{R}, q^{\epsilon}\right)$ with $\mathcal{H}\left(\mathcal{R}, q^{0}\right)=\mathbb{C}[W]$. To relate representations of these algebras we use their analytic localizations, as in Section 1.5.

Let $c_{\alpha, \epsilon}$ be the $c$-function with respect to $\left(\mathcal{R}, q^{\epsilon}\right)$, as in (1.6). For $\epsilon \in[-1,1] \backslash\{0\}$ the ball $\epsilon B \subset \mathfrak{t}$ satisfies the conditions 2.1.1 with respect to $u c^{\epsilon} \in T$ and the parameter function $q^{\epsilon}$ (which enters via the function $c_{\alpha, \epsilon}$ ). For $\epsilon=0$ the point $\epsilon B=\{0\}$ trivially fulfills all of the conditions, except that it is not open. We write

$$
U_{\epsilon}=W_{0} u c^{\epsilon} \exp (\epsilon B) \quad \epsilon \in[-1,1]
$$

and we abbreviate $U:=U_{1}$. We define a $W_{0}$-equivariant scaling map,

$$
\sigma_{\epsilon}: U \rightarrow U_{\epsilon}, w(u c \exp (b)) \mapsto w\left(u c^{\epsilon} \exp (\epsilon b)\right) .
$$


As was noted in Opd2, Lemma 5.1], $\sigma_{\epsilon}$ is an analytic diffeomorphism for $\epsilon \neq 0$, while $\sigma_{0}$ is a locally constant map with range $U_{0}=W_{0} u$. Let $\imath_{w, \epsilon}^{0}$ be the element constructed in Proposition 1.5.1. By (1.26) $\sigma_{\epsilon}$ induces an algebra isomorphism

$$
\begin{aligned}
\rho_{\epsilon}: \mathcal{H}_{\epsilon}^{m e}\left(U_{\epsilon}\right) & \rightarrow \mathcal{H}^{m e}(U) \\
f l_{w, \epsilon}^{0} & \mapsto\left(f \circ \sigma_{\epsilon}\right) \imath_{w}^{0},
\end{aligned} \quad \epsilon \in[-1,1] \backslash\{0\},
$$

where $f \in C^{m e}(U)$ and $w \in W_{0}$. We will show that these maps depend continuously on $\epsilon$ and have a well-defined limit as $\epsilon \rightarrow 0$.

Lemma 4.1.1. For $\epsilon \in[-1,1] \backslash\{0\}$ and $\alpha \in R_{0}$ write $d_{\alpha, \epsilon}=\left(c_{\alpha, \epsilon} \circ \sigma_{\epsilon}\right) c_{\alpha}^{-1} \in$ $C^{m e}(U)$. This defines a bounded invertible analytic function on $U \times([-1,1] \backslash\{0\})$, which extends to a function on $\bar{U} \times[-1,1]$ with the same properties.

Proof. This extends Opd2, Lemma 5.2] to $\epsilon=0$. By the definition (1.6)

$$
\begin{aligned}
d_{\alpha, \epsilon}(t)=\frac{1+\theta_{-\alpha}(t)}{1+\theta_{-\alpha}\left(\sigma_{\epsilon}(t)\right)} \frac{1+q\left(s_{\alpha}\right)^{-\epsilon / 2} q\left(t_{\alpha} s_{\alpha}\right)^{\epsilon / 2} \theta_{-\alpha}\left(\sigma_{\epsilon}(t)\right)}{1+q\left(s_{\alpha}\right)^{-1 / 2} q\left(t_{\alpha} s_{\alpha}\right)^{1 / 2} \theta_{-\alpha}(t)} \\
\quad \times \frac{1-\theta_{-\alpha}(t)}{1-\theta_{-\alpha}\left(\sigma_{\epsilon}(t)\right)} \frac{1-q\left(s_{\alpha}\right)^{-\epsilon / 2} q\left(t_{\alpha} s_{\alpha}\right)^{-\epsilon / 2} \theta_{-\alpha}\left(\sigma_{\epsilon}(t)\right)}{1-q\left(s_{\alpha}\right)^{-1 / 2} q\left(t_{\alpha} s_{\alpha}\right)^{-1 / 2} \theta_{-\alpha}(t)} .
\end{aligned}
$$

Let us abbreviate $d_{\alpha, \epsilon}=\frac{f_{1} f_{2}}{g_{1} g_{2}} \times \frac{f_{3} f_{4}}{g_{3} g_{4}}$. We see that $d_{\alpha, \epsilon}(t)$ extends to an invertible analytic function on $\bar{U} \times[-1,1]$ if none of the quotients $f_{n} / g_{n}$ has a zero or a pole on this domain. By Condition 2.1.1.c there is a unique $b \in w(\log (c)+\bar{B})$ such that $t=w(u) \exp (b)$. This defines a coordinate system on $w(u c) \exp (\bar{B})$, and $\sigma_{\epsilon}(t)=w(u) \exp (\epsilon b)$. By Condition 2.1.1 d, if either $f_{n}(t)=0$ or $g_{n}(t)=0$ for some $t \in w(u c \exp (\bar{B})) \subset \bar{U}$, then $f_{n}(w(u c))=g_{n}(w(u c))=0$. One can easily check that, in this situation,

$$
\frac{f_{n}(t)}{g_{n}(t)}=\left(\frac{1-e^{-\alpha(b) \epsilon}}{1-e^{-\alpha(b)}}\right)^{(-1)^{n}} .
$$

Again, by Condition 2.1.1 d the only critical points of this function are those for which $\alpha(b)=0$. For $\epsilon \neq 0$ both the numerator and the denominator have a zero of order 1 at such points, so the singularity is removable. For the case $\epsilon=0$ we need to have a closer look. In our new coordinate system we can write

$$
\begin{aligned}
c_{\alpha, \epsilon}\left(\sigma_{\epsilon}(t)\right)= & \frac{f_{2}(t)}{g_{1}(t)} \frac{f_{4}(t)}{g_{3}(t)} \\
= & \frac{u\left(w^{-1} \alpha\right)+\left(q\left(s_{\alpha}\right)^{-1 / 2} q\left(t_{\alpha} s_{\alpha}\right)^{1 / 2} e^{-\alpha(b)}\right)^{\epsilon}}{u\left(w^{-1} \alpha\right)+e^{-\alpha(b) \epsilon}} \\
& \times \frac{u\left(w^{-1} \alpha\right)-\left(q\left(s_{\alpha}\right)^{-1 / 2} q\left(t_{\alpha} s_{\alpha}\right)^{-1 / 2} e^{-\alpha(b)}\right)^{\epsilon}}{u\left(w^{-1} \alpha\right)-e^{-\alpha(b) \epsilon}} .
\end{aligned}
$$

What happens when $\epsilon$ goes to 0 depends on $u\left(w^{-1} \alpha\right)$. The rational function $f_{2}(t) / g_{1}(t)$ has no zeros or poles in a neighborhood of $\epsilon=0$ if $u\left(w^{-1} \alpha\right) \neq-1$, so then $\lim _{\epsilon \rightarrow 0} f_{2}(t) / g_{1}(t)=1$. For $u\left(w^{-1} \alpha\right)=-1$ we have $f_{2}(t)=g_{1}(t)=0$, so 
by L'Hospital's rule

$$
\begin{aligned}
& \lim _{\epsilon \rightarrow 0} \frac{f_{2}(t)}{g_{1}(t)}=\lim _{\epsilon \rightarrow 0} \frac{\frac{\partial}{\partial \epsilon} f_{2}(t)}{\frac{\partial}{\partial \epsilon} g_{1}(t)}=\lim _{\epsilon \rightarrow 0} \frac{\log \left(q\left(s_{\alpha}\right)^{-1 / 2} q\left(t_{\alpha} s_{\alpha}\right)^{1 / 2} e^{-\alpha(b)}\right)}{-\alpha(b)} \\
& \times \frac{\left(q\left(s_{\alpha}\right)^{-1 / 2} q\left(t_{\alpha} s_{\alpha}\right)^{1 / 2} e^{-\alpha(b)}\right)^{\epsilon}}{e^{-\alpha(b) \epsilon}}=\frac{-\log q\left(s_{\alpha}\right) / 2+\log q\left(t_{\alpha} s_{\alpha}\right) / 2-\alpha(b)}{-\alpha(b)} .
\end{aligned}
$$

Similarly $f_{4}(t) / g_{3}(t)$ has no zeros or poles in a neighborhood of $\epsilon=0$ if $u\left(w^{-1} \alpha\right) \neq$ 1 , so $\lim _{\epsilon \rightarrow 0} f_{4}(t) / g_{3}(t)=1$ in that case. For $u\left(w^{-1} \alpha\right)=1$ we have $f_{4}(t)=g_{3}(t)=$ 0 , so again by L'Hopital:

$$
\begin{aligned}
& \lim _{\epsilon \rightarrow 0} \frac{f_{4}(t)}{g_{3}(t)}=\lim _{\epsilon \rightarrow 0} \frac{\frac{\partial}{\partial \epsilon} f_{4}(t)}{\frac{\partial}{\partial \epsilon} g_{3}(t)}=\lim _{\epsilon \rightarrow 0} \frac{-\log \left(q\left(s_{\alpha}\right)^{-1 / 2} q\left(t_{\alpha} s_{\alpha}\right)^{-1 / 2} e^{-\alpha(b)}\right)}{\alpha(b)} \\
& \quad \times \frac{\left(q\left(s_{\alpha}\right)^{-1 / 2} q\left(t_{\alpha} s_{\alpha}\right)^{-1 / 2} e^{-\alpha(b)}\right)^{\epsilon}}{e^{-\alpha(b) \epsilon}}=\frac{\log q\left(s_{\alpha}\right) / 2+\log q\left(t_{\alpha} s_{\alpha}\right) / 2+\alpha(b)}{\alpha(b)} .
\end{aligned}
$$

Summarizing, we have

$$
\lim _{\epsilon \rightarrow 0} c_{\alpha, \epsilon}\left(\sigma_{\epsilon}(t)\right)=\left\{\begin{array}{cll}
1 & \text { if } u\left(w^{-1} \alpha\right)^{2} \neq 1, \\
\frac{\alpha(b)+\left(\log q\left(s_{\alpha}\right)-\log q\left(t_{\alpha} s_{\alpha}\right)\right) / 2}{\alpha(b)} & \text { if } u\left(w^{-1} \alpha\right)=-1, \\
\frac{\alpha(b)+\left(\log q\left(s_{\alpha}\right)+\log q\left(t_{\alpha} s_{\alpha}\right)\right) / 2}{\alpha(b)} & \text { if } u\left(w^{-1} \alpha\right)=1 .
\end{array}\right.
$$

For later use we record that with (2.3) we can interpret this as

$$
\text { (4.4) } \lim _{\epsilon \rightarrow 0} c_{\alpha, \epsilon}\left(\sigma_{\epsilon}(w(u) \exp (b))\right)=\left(k_{w(u), \alpha}+\alpha(b)\right) / \alpha(b)=\tilde{c}_{\alpha}(b) \quad \alpha \in R_{w(u)} \text {. }
$$

Now we know that at least $d_{\alpha, 0}=\lim _{\epsilon \rightarrow 0} d_{\alpha, \epsilon}$ exists as a meromorphic function on $\bar{U}$. For $u\left(w^{-1} \alpha\right)^{2} \neq 1, d_{\alpha, 0}=c_{\alpha}^{-1}$ is invertible by Condition 2.1.1, d. For $u\left(w^{-1} \alpha\right)=-1$ we have

$d_{\alpha, 0}(t)=\frac{1-e^{-\alpha(b)}}{\alpha(b)} \frac{\alpha(b)+\log \left(q\left(s_{\alpha}\right) / q\left(t_{\alpha} s_{\alpha}\right)\right) / 2}{1-q\left(s_{\alpha}\right)^{-1 / 2} q\left(t_{\alpha} s_{\alpha}\right)^{1 / 2} e^{-\alpha(b)}} \frac{1+e^{-\alpha(b)}}{1+q\left(s_{\alpha}\right)^{-1 / 2} q\left(t_{\alpha} s_{\alpha}\right)^{-1 / 2} e^{-\alpha(b)}}$,

while for $u\left(w^{-1} \alpha\right)=1$ we have

$d_{\alpha, 0}(t)=\frac{1-e^{-\alpha(b)}}{\alpha(b)} \frac{1+e^{-\alpha(b)}}{1+q\left(s_{\alpha}\right)^{-1 / 2} q\left(t_{\alpha} s_{\alpha}\right)^{1 / 2} e^{-\alpha(b)}} \frac{\alpha(b)+\log \left(q\left(s_{\alpha}\right) q\left(t_{\alpha} s_{\alpha}\right)\right) / 2}{1-q\left(s_{\alpha}\right)^{-1 / 2} q\left(t_{\alpha} s_{\alpha}\right)^{-1 / 2} e^{-\alpha(b)}}$.

These expressions define invertible functions by Condition 2.1.1 c. We conclude that $d_{\alpha, \epsilon}(t)$ and $d_{\alpha, \epsilon}^{-1}(t)$ are indeed analytic functions on $\bar{U} \times[-1,1]$. Since this domain is compact, they are bounded.

Lemma 4.1.1 enables us to show that the maps $\rho_{\epsilon}$ preserve analyticity:

Proposition 4.1.2. The maps (4.3) restrict to isomorphisms of topological algebras

$$
\rho_{\epsilon}: \mathcal{H}_{\epsilon}^{a n}\left(U_{\epsilon}\right) \stackrel{\sim}{\longrightarrow} \mathcal{H}^{a n}(U) .
$$

There is a well-defined limit homomorphism

$$
\rho_{0}=\lim _{\epsilon \rightarrow 0} \rho_{\epsilon}: \mathbb{C}[W] \rightarrow \mathcal{H}^{a n}(U)
$$

such that for every $w \in W$ the map

$$
[-1,1] \rightarrow \mathcal{H}^{a n}(U): \epsilon \mapsto \rho_{\epsilon}\left(N_{w}\right)
$$


is analytic.

Proof. The first statement is Opd2, Theorem 5.3], but for the remainder we need to prove this anyway. It is clear that $\rho_{\epsilon}$ restricts to an isomorphism between $C^{a n}\left(U_{\epsilon}\right)$ and $C^{a n}(U)$. For a simple reflection $s_{\alpha} \in S_{0}$ corresponding to $\alpha \in F_{0}$ we have

$$
\begin{aligned}
N_{s}+q(s)^{-\epsilon / 2} & =q(s)^{\epsilon / 2} c_{\alpha, \epsilon}\left(\imath_{s, \epsilon}^{0}+1\right) \\
\rho_{\epsilon}\left(N_{s}\right) & =q(s)^{\epsilon / 2}\left(c_{\alpha, \epsilon} \circ \sigma_{\epsilon}\right)\left(l_{s}^{0}+1\right)-q(s)^{-\epsilon / 2} \\
& =q(s)^{(\epsilon-1) / 2}\left(c_{\alpha, \epsilon} \circ \sigma_{\epsilon}\right) c_{\alpha}^{-1}\left(N_{s}+q(s)^{-1 / 2}\right)-q(s)^{-\epsilon / 2} \\
& =q(s)^{(\epsilon-1) / 2} d_{\alpha, \epsilon}\left(N_{s}+q(s)^{-1 / 2}\right)-q(s)^{-\epsilon / 2} .
\end{aligned}
$$

By Lemma 4.1.1 such elements are analytic in $\epsilon \in[-1,1]$ and $t \in U$, so in particular they belong to $\mathcal{H}^{a n}(U)$. Moreover, since every $d_{\alpha, \epsilon}$ is invertible and by (1.26), the set $\left\{\rho_{\epsilon}\left(N_{w}\right): w \in W_{0}\right\}$ is a basis for $\mathcal{H}^{a n}(U)$ as a $C^{a n}(U)$-module. Therefore $\rho_{\epsilon}$ restricts to an isomorphism between the topological algebras $\mathcal{H}_{\epsilon}^{a n}\left(U_{\epsilon}\right)$ and $\mathcal{H}^{a n}(U)$ for $\epsilon \neq 0$.

Given any $w \in W$, there is a unique $x \in X^{+}$such that $w \in W_{0} x W_{0}$. By Lemma 1.2.2 there exist unique coefficients $c_{w, u, v}(q) \in \mathbb{C}$ such that

$$
N_{w}=\sum_{u \in W^{x}, v \in W_{0}} c_{w, u, v}(q) N_{u} \theta_{x} N_{v} .
$$

From (1.4) we see that in fact $c_{w, u, v}(q) \in \mathbb{Q}\left(\left\{q(s)^{1 / 2}: s \in S^{\text {aff }}\right\}\right)$, so in particular these coefficients depend analytically on $q$. Moreover, $\rho_{\epsilon}\left(\theta_{x}\right)=\theta_{x} \circ \sigma_{\epsilon}$ depends analytically on $\epsilon$, as a function on $U$, so

$$
\rho_{\epsilon}\left(N_{w}\right)=\sum_{u \in W^{x}, v \in W_{0}} c_{w, u, v}\left(q^{\epsilon}\right) \rho_{\epsilon}\left(N_{u}\right)\left(\theta_{x} \circ \rho_{\epsilon}\right) \rho_{\epsilon}\left(N_{v}\right)
$$

is analytic in $\epsilon \in[-1,1]$. Thus $\rho_{0}$ exists as a linear map. But, being a limit of algebra homomorphisms, it must also be multiplicative.

Suppose that $u \in T_{u n}$ is $W_{0}$-invariant, so that $R_{u}=R_{0}$ and

$$
\exp _{u}: W_{0}(\log (c)+B) \rightarrow U=u W_{0} c \exp (B)
$$

is a $W_{0}$-equivariant bijection. Then clearly

$$
\sigma_{\epsilon} \circ \exp _{u}=\exp _{u} \circ \lambda_{\epsilon}: \epsilon W_{0}(\log (c)+B) \rightarrow U_{\epsilon} .
$$

Let $\Phi_{u}$ be as in (2.5), with $V=W_{0} \log (c)+B$. It follows from (4.6), (4.1) and (4.3) that

$$
m_{\epsilon} \circ \Phi_{u, \epsilon}=\Phi_{u} \circ \rho_{\epsilon} \quad \epsilon \in[-1,1] \backslash\{0\}
$$

as maps $\mathcal{H}_{\epsilon}^{m e}\left(U_{\epsilon}\right) \rightarrow C^{m e}\left(W_{0} \log (c)+B\right)^{W_{0}} \otimes_{Z\left(\mathbb{H}\left(\tilde{\mathcal{R}}_{u}, k_{u}\right)\right)} \mathbb{H}\left(\tilde{\mathcal{R}}_{u}, k_{u}\right)$. The maps $\Phi_{u, \epsilon}$ can also be defined for $\epsilon=0$, simply by

$$
\Phi_{u, 0}\left(f N_{w}\right)=\left(f \circ \exp _{u}\right) w \in C^{a n}(\mathfrak{t})^{W_{0}} \otimes_{Z\left(\mathbb{H}\left(\tilde{\mathcal{R}}_{u}, k_{u}\right)\right)} \mathbb{H}\left(\tilde{\mathcal{R}}_{u}, k_{u}\right),
$$

where $f \in C^{a n}(T)$ and $w \in W_{0}$. By (4.5) for $\alpha \in F_{0}$,

$$
\begin{aligned}
m_{\epsilon} \circ \Phi_{u, \epsilon}\left(N_{s_{\alpha}}\right) & =m_{\epsilon} \circ \Phi_{u, \epsilon}\left(q\left(s_{\alpha}\right)^{\epsilon / 2} c_{\alpha, \epsilon}\left(\imath_{s, \epsilon}^{0}+1\right)-q\left(s_{\alpha}\right)^{\epsilon / 2}\right) \\
& =m_{\epsilon}\left(q\left(s_{\alpha}\right)^{\epsilon / 2}\left(c_{\alpha, \epsilon} \circ \exp _{u}\right)\left(\tilde{\imath}_{s_{\alpha}, \epsilon}+1\right)-q\left(s_{\alpha}\right)^{\epsilon / 2}\right) \\
& =q\left(s_{\alpha}\right)^{\epsilon / 2}\left(c_{\alpha, \epsilon} \circ \exp _{u} \circ \lambda_{\epsilon}\right)\left(\tilde{s}_{s_{\alpha}}+1\right)-q\left(s_{\alpha}\right)^{\epsilon / 2} \\
& =q\left(s_{\alpha}\right)^{\epsilon / 2}\left(c_{\alpha, \epsilon} \circ \exp _{u} \circ \lambda_{\epsilon}\right) \tilde{c}_{\alpha}^{-1}\left(s_{\alpha}+1\right)-q\left(s_{\alpha}\right)^{\epsilon / 2} .
\end{aligned}
$$


Since $R_{u}=R_{0}$, (4.4) tells us that $\lim _{\epsilon \rightarrow 0}\left(c_{\alpha, \epsilon} \circ \exp _{u} \circ \lambda_{\epsilon}\right) \tilde{c}_{\alpha}^{-1}=1$. Hence

$$
\lim _{\epsilon \rightarrow 0} m_{\epsilon} \circ \Phi_{u, \epsilon}\left(N_{s_{\alpha}}\right)=s_{\alpha}=m_{0} \circ \Phi_{u, 0}\left(N_{s_{\alpha}}\right) .
$$

On the other hand, it is clear that for $f \in \mathbb{C}[X] \cong \mathcal{O}(T)$,

$$
\lim _{\epsilon \rightarrow 0} m_{\epsilon} \circ \Phi_{u, \epsilon}(f)=\lim _{\epsilon \rightarrow 0} f \circ \exp _{u} \circ \lambda_{\epsilon}=f(u)=m_{0} \circ \Phi_{u, 0}(f) .
$$

Since $\rho_{0}=\lim _{\epsilon \rightarrow 0} \rho_{\epsilon}$ exists, we can conclude that

$$
m_{0} \circ \Phi_{u, 0}=\Phi_{u} \circ \rho_{0}: \mathbb{C}\left[X \rtimes W_{0}\right] \rightarrow \mathbb{H}\left(\tilde{\mathcal{R}}_{u}, k_{u}\right) .
$$

4.2. Preserving unitarity. Proposition 4.1.2 shows that for $\epsilon \in[-1,1] \backslash\{0\}$ there is an equivalence between the categories $\operatorname{Mod}_{f, U}(\mathcal{H})$ and $\operatorname{Mod}_{f, U_{\epsilon}}\left(\mathcal{H}_{\epsilon}\right)$. It would be nice if this equivalence would preserve unitarity, but that is not automatic. In fact, these categories are not always closed under taking duals of $\mathcal{H}$-representations.

From (3.3) we see that an $\mathcal{H}$-representation with central character $W_{0} t$ can only be unitary if $\overline{t^{-1}} \in W_{0} t$, where $\overline{t^{-1}}(x)=\overline{t(-x)}$ for $x \in X$. To define a * on $\mathcal{H}^{a n}(U)$ we must thus replace $U$ by

$$
U^{ \pm 1}:=U \cup\left\{t^{-1}: t \in U\right\}
$$

Let $\pm W_{0}$ be the group $\{ \pm 1\} \times W_{0}$, which acts on $T$ by $-w(t)=w(t)^{-1}$. The above means that we need the following strengthening of Condition 2.1.1.e:

$\left(\mathrm{e}^{\prime}\right)$ As Condition 2.1.1.e, but with $\pm W_{0} \rtimes \Gamma$ instead of $W_{0}^{\prime}$.

Lemma 4.1.1 and Proposition 4.1.2 remain valid under this condition, with the same proof. Equations (3.3) and (1.22) show that the involution from $\mathcal{H}$ extends naturally to $\mathcal{H}^{m e}\left(U^{ \pm 1}\right) \rtimes \Gamma$ by

$$
\left(N_{\gamma} f\right)^{*}=N_{w_{0}}\left(f \circ-w_{0}\right) N_{w_{0}}^{-1} N_{\gamma^{-1}} \quad \gamma \in W_{0}^{\prime}, f \in C^{m e}\left(U^{ \pm 1}\right),
$$

where $w_{0}$ is the longest element of $W_{0}$. According to Opd2, (4.56)]

$$
\left(\imath_{w}^{0}\right)^{*}=N_{w_{0}} \prod_{\alpha \in R_{0}^{+} \cap w^{\prime} R_{0}^{-}} \frac{c_{\alpha}}{c_{-\alpha}} \imath_{w^{\prime}}^{0} N_{w_{0}}^{-1},
$$

where $w \in W_{0}$ and $w^{\prime}=w_{0} w^{-1} w_{0}$. We extend the map from Proposition 4.1.2 to $\rho_{\epsilon}: \mathcal{H}_{\epsilon}^{a n}\left(U_{\epsilon}\right) \rtimes \Gamma \rightarrow \mathcal{H}^{a n}(U) \rtimes \Gamma$ by defining $\rho_{\epsilon}\left(N_{\gamma}\right)=N_{\gamma}$ for $\gamma \in \Gamma$. Usually the maps $\rho_{\epsilon}$ do not preserve the ${ }^{*}$, but this can be fixed. For $\epsilon \in[-1,1]$ consider the element

$$
M_{\epsilon}=\rho_{\epsilon}\left(N_{w_{0}, \epsilon}^{-1}\right)^{*} N_{w_{0}} \prod_{\alpha \in R_{0}^{+}} d_{\alpha, \epsilon} \in \mathcal{H}^{a n}(U) .
$$

We will use $M_{\epsilon}$ to extend Opd2, Corollary 5.7]. However, this result contained a small mistake: the element $A_{\epsilon}$ in $\mathrm{Opd} 2$ is not entirely correct, we replace it by $M_{\epsilon}$.

Theorem 4.2.1. For all $\epsilon \in[-1,1]$ the element $M_{\epsilon} \in \mathcal{H}^{a n}\left(U^{ \pm 1}\right)$ is invertible, positive and bounded. It has a positive square root $M_{\epsilon}^{1 / 2}$ and the map $\epsilon \mapsto M_{\epsilon}^{1 / 2}$ is analytic. The map

$$
\tilde{\rho}_{\epsilon}: \mathcal{H}_{\epsilon}^{a n}\left(U_{\epsilon}\right) \rtimes \Gamma \rightarrow \mathcal{H}^{a n}\left(U^{ \pm 1}\right) \rtimes \Gamma, h \mapsto M_{\epsilon}^{1 / 2} \rho_{\epsilon}(h) M_{\epsilon}^{-1 / 2}
$$

is a homomorphism of topological *algebras, and an isomorphism if $\epsilon \neq 0$. For any $w \in W \rtimes \Gamma$ the map

$$
[-1,1] \rightarrow \mathcal{H}^{a n}\left(U^{ \pm 1}\right) \rtimes \Gamma: \epsilon \mapsto \tilde{\rho}_{\epsilon}\left(N_{w}\right)
$$

is analytic. 
Proof. By Lemma 4.1.1 and Proposition 4.1.2 the $M_{\epsilon}$ are invertible, bounded and analytic in $\epsilon$. Consider, for $\epsilon \neq 0$, the automorphism $\mu_{\epsilon}$ of $\mathcal{H}^{m e}\left(U^{ \pm 1}\right)$ given by

$$
\mu_{\epsilon}(h)=\rho_{\epsilon}\left(\rho_{\epsilon}^{-1}(h)^{*}\right)^{*} .
$$

We will discuss its effect on three kinds of elements. First, for $f \in C^{m e}\left(U^{ \pm 1}\right)$ we have, by (4.8) and the $W_{0}$-equivariance of $\sigma_{\epsilon}$ :

$$
\begin{aligned}
\mu_{\epsilon}(f) & =\rho_{\epsilon}\left(\left(f \circ \sigma_{\epsilon}\right)^{*}\right)^{*} \\
& =\rho_{\epsilon}\left(N_{w_{0}}\left(f \circ\left(-w_{0}\right) \circ \sigma_{\epsilon}\right) N_{w_{0}, \epsilon}^{-1}\right)^{*} \\
& =\rho_{\epsilon}\left(N_{w_{0}, \epsilon}^{-1}\right)^{*}\left(f \circ-w_{0}\right)^{*} \rho_{\epsilon}\left(N_{w_{0}, \epsilon}\right)^{*} \\
& =\rho_{\epsilon}\left(N_{w_{0}, \epsilon}^{-1}\right)^{*} N_{w_{0}} f N_{w_{0}}^{-1} \rho_{\epsilon}\left(N_{w_{0}, \epsilon}\right)^{*} \\
& =\rho_{\epsilon}\left(N_{w_{0}, \epsilon}^{-1}\right)^{*} N_{w_{0}} \prod_{\alpha \in R_{0}^{+}} d_{\alpha, \epsilon} f \prod_{\alpha \in R_{0}^{+}}^{-1} d_{\alpha, \epsilon}^{-1} N_{w_{0}}^{-1} \rho_{\epsilon}\left(N_{w_{0}, \epsilon}\right)^{*} \\
& =M_{\epsilon} f M_{\epsilon}^{-1} .
\end{aligned}
$$

Second, suppose that the simple reflections $s$ and $s^{\prime}=w_{0} s w_{0} \in S_{0}$ correspond to $\alpha$ and $\alpha^{\prime}=-w_{0} \alpha \in F_{0}$. Using Proposition 1.5.1.b for $\mathcal{H}_{\epsilon}^{m e}\left(U^{ \pm 1}\right)$ and (4.9) we find

$$
\begin{aligned}
M_{\epsilon} \imath_{s}^{0} M_{\epsilon}^{-1} & =\rho_{\epsilon}\left(N_{w_{0}, \epsilon}^{-1}\right)^{*} N_{w_{0}} \prod_{\beta \in R_{0}^{+}} d_{\beta, \epsilon} l_{s}^{0} \prod_{\beta \in R_{0}^{+}} d_{\beta, \epsilon}^{-1} N_{w_{0}}^{-1} \rho_{\epsilon}\left(N_{w_{0}, \epsilon}\right)^{*} \\
& =\rho_{\epsilon}\left(N_{w_{0}, \epsilon}^{-1}\right)^{*} N_{w_{0}} \imath_{s}^{0} d_{\alpha, \epsilon}^{-1} d_{-\alpha, \epsilon} N_{w_{0}}^{-1} \rho_{\epsilon}\left(N_{w_{0}, \epsilon}\right)^{*} \\
& =\rho_{\epsilon}\left(N_{w_{0}, \epsilon}^{-1}\right)^{*} N_{w_{0}} \imath_{s}^{0} \frac{c_{-\alpha}}{c_{\alpha}} N_{w_{0}}^{-1} N_{w_{0}} \frac{c_{\alpha, \epsilon} \circ \sigma_{\epsilon}}{c_{-\alpha, \epsilon} \circ \sigma_{\epsilon}} N_{w_{0}}^{-1} \rho_{\epsilon}\left(N_{w_{0}, \epsilon}\right)^{*} \\
& =\rho_{\epsilon}\left(N_{w_{0}, \epsilon}^{-1}\right)^{*}\left(\imath_{s^{\prime}}^{0}\right)^{*}\left(\frac{c_{\alpha^{\prime}, \epsilon} \circ \sigma_{\epsilon}}{c_{-\alpha^{\prime}, \epsilon} \circ \sigma_{\epsilon}}\right)^{*} \rho_{\epsilon}\left(N_{w_{0}, \epsilon}\right)^{*} \\
& =\left(\rho_{\epsilon}\left(N_{w_{0}, \epsilon}\right) \frac{c_{\alpha^{\prime}, \epsilon} \circ \sigma_{\epsilon}}{c_{-\alpha^{\prime}, \epsilon} \circ \sigma_{\epsilon}} l_{s^{\prime}}^{0} \rho_{\epsilon}\left(N_{w_{0}, \epsilon}^{-1}\right)\right)^{*} \\
& =\rho_{\epsilon}\left(N_{w_{0}} \frac{c_{\alpha^{\prime}}}{c_{-\alpha^{\prime}}} l_{s^{\prime}, \epsilon}^{0} N_{w_{0}, \epsilon}^{-1}\right)^{*} \\
& =\rho_{\epsilon}\left(\left(l_{s, \epsilon}^{0}\right)^{*}\right)^{*}=\mu_{\epsilon}\left(l_{s}^{0}\right) .
\end{aligned}
$$

Third, for $\gamma \in \Gamma$ by definition

$$
\mu_{\epsilon}\left(N_{\gamma}\right)=\rho_{\epsilon}\left(\rho_{\epsilon}^{-1}\left(N_{\gamma}\right)^{*}\right)^{*}=\rho_{\epsilon}\left(N_{\gamma}^{-1}\right)^{*}=\left(N_{\gamma}^{-1}\right)^{*}=N_{\gamma} .
$$

Since elements of the above three types generate $\mathcal{H}^{m e}\left(U^{ \pm 1}\right) \rtimes \Gamma$, we conclude that

$$
\mu_{\epsilon}(h)=M_{\epsilon} h M_{\epsilon}^{-1} \quad \text { for all } h \in \mathcal{H}^{m e}\left(U^{ \pm 1}\right) \rtimes \Gamma .
$$

Now we can see that

$$
\begin{aligned}
\rho_{\epsilon}\left(N_{w_{0}, \epsilon}^{-1}\right)^{*} & =\rho_{\epsilon}\left(\left(N_{w_{0}, \epsilon}^{*}\right)^{-1}\right)^{*}=\rho_{\epsilon}\left(\left(N_{w_{0}, \epsilon}^{-1}\right)^{*}\right)^{*} \\
& =\mu_{\epsilon}\left(\rho_{\epsilon}\left(N_{w_{0}, \epsilon}^{-1}\right)\right)=M_{\epsilon} \rho_{\epsilon}\left(N_{w_{0}, \epsilon}^{-1}\right) M_{\epsilon}^{-1} \\
N_{e} & =M_{\epsilon}^{-1} \rho_{\epsilon}\left(N_{w_{0}, \epsilon}^{-1}\right)^{*} N_{w_{0}} \prod_{\alpha \in R_{0}^{+}} d_{\alpha, \epsilon}=\rho_{\epsilon}\left(N_{w_{0}, \epsilon}^{-1}\right) M_{\epsilon}^{-1} N_{w_{0}} \prod_{\alpha \in R_{0}^{+}} d_{\alpha, \epsilon}, \\
M_{\epsilon} & =N_{w_{0}} \prod_{\alpha \in R_{0}^{+}} d_{\alpha, \epsilon} \rho_{\epsilon}\left(N_{w_{0}, \epsilon}^{-1}\right)=\left(\rho_{\epsilon}\left(N_{w_{0}, \epsilon}^{-1}\right)^{*}\left(N_{w_{0}} \prod_{\alpha \in R_{0}^{+}} d_{\alpha, \epsilon}\right)^{*}\right)^{*} \\
& =\left(\rho_{\epsilon}\left(N_{w_{0}, \epsilon}^{-1}\right)^{*} N_{w_{0}} \prod_{\alpha \in R_{1}^{+}} d_{\alpha, \epsilon}\right)^{*}=M_{\epsilon}^{*} .
\end{aligned}
$$

Thus the elements $M_{\epsilon}$ are Hermitian $\forall \epsilon \neq 0$. By continuity in $\epsilon, M_{0}$ is also Hermitian. Moreover, they are all invertible, and $M_{1}=N_{e}$, so they are in fact strictly positive. We already knew that the element $\epsilon \mapsto M_{\epsilon}$ of

$$
C^{a n}\left([-1,1] ; \mathcal{H}^{a n}\left(U^{ \pm 1}\right)\right) \cong C^{a n}\left([-1,1] \times U^{ \pm 1}\right) \otimes_{\mathcal{A}} \mathcal{H}
$$


is bounded, so we can construct its square root using holomorphic functional calculus in the Fréchet algebra $C_{b}^{a n}\left([-1,1] \times U^{ \pm 1}\right) \otimes_{\mathcal{A}} \mathcal{H}$, where the subscript $b$ denotes bounded functions. This ensures that $\epsilon \rightarrow M_{\epsilon}^{1 / 2}$ is still analytic. Finally, for $\epsilon \neq 0$,

$$
\begin{aligned}
\tilde{\rho}_{\epsilon}(h)^{*} & =\left(M_{\epsilon}^{1 / 2} \rho_{\epsilon}(h) M_{\epsilon}^{-1 / 2}\right)^{*} \\
& =M_{\epsilon}^{-1 / 2} \rho_{\epsilon}(h)^{*} M_{\epsilon}^{1 / 2} \\
& =M_{\epsilon}^{-1 / 2} \mu_{\epsilon}\left(\rho_{\epsilon}\left(h^{*}\right)\right) M_{\epsilon}^{1 / 2} \\
& =M_{\epsilon}^{1 / 2} \rho_{\epsilon}\left(h^{*}\right) M_{\epsilon}^{-1 / 2}=\tilde{\rho}_{\epsilon}\left(h^{*}\right) .
\end{aligned}
$$

Again this extends to $\epsilon=0$ by continuity.

Corollary 4.2.2. For $u c \in U$ and $\epsilon \in[-1,1]$ there is a family of additive functors

$$
\begin{aligned}
& \tilde{\sigma}_{\epsilon, u c}: \operatorname{Mod}_{f, W_{0}^{\prime} u c}(\mathcal{H} \rtimes \Gamma) \rightarrow \operatorname{Mod}_{f, W_{0}^{\prime} \sigma_{\epsilon}(u c)}\left(\mathcal{H}_{\epsilon} \rtimes \Gamma\right), \\
& (\pi, V) \mapsto\left(\pi \circ \tilde{\rho}_{\epsilon}, V\right)
\end{aligned}
$$

with the following properties:

(a) for all $w \in W \rtimes \Gamma$ and $(\pi, V)$ the map $[-1,1] \rightarrow \operatorname{End}_{\mathbb{C}}(V): \epsilon \mapsto \tilde{\sigma}_{\epsilon, u c}(\pi)\left(N_{w}\right)$ is analytic;

(b) for $\epsilon \neq 0, \tilde{\sigma}_{\epsilon, u c}$ is an equivalence of categories;

(c) $\tilde{\sigma}_{\epsilon, u c}$ preserves unitarity;

(d) for $\epsilon<0, \tilde{\sigma}_{\epsilon, u c}$ exchanges tempered and anti-tempered modules, where antitempered means that $|s|^{-1} \in T^{-}$for all $\mathcal{A}$-weights $s \in T$;

(e) for $\epsilon \geq 0, \tilde{\sigma}_{\epsilon, u c}$ preserves temperedness;

(f) for $\epsilon>0, \tilde{\sigma}_{\epsilon, u c}$ preserves the discrete series.

Proof. Parts (a), (b) and (c) follow immediately from Theorem 4.2.1, Let $(\pi, V)$ be a finite dimensional $\mathcal{H}^{a n}\left(U^{ \pm 1}\right) \rtimes \Gamma$-representation. Conjugation by $M_{\epsilon}^{1 / 2}$ does not change the isomorphism class of $\pi$, so $\tilde{\sigma}_{\epsilon, u c}(\pi)$ has the same $\mathcal{A}_{\epsilon}$-weights as $\pi \circ \rho_{\epsilon}$, which by construction are $\sigma_{\epsilon}$ of the $\mathcal{A}$-weights of $\pi$. Now parts (d), (e) and (f) are obvious consequences of $\left|\sigma_{\epsilon}(t)\right|=|t|^{\epsilon}$.

As the notation indicates, $\tilde{\sigma}_{\epsilon, u c}$ depends on the previously chosen base point $u c$. For one $t \in T$ there can exist several possible base points such that $t \in U$, and these could in principle give rise to different functors $\tilde{\sigma}_{\epsilon, t}$. This ambiguity disappears if we restrict to $t=u c$ in Corollary 4.2.2. Then

$$
\tilde{\sigma}_{\epsilon}:=\bigoplus_{t \in T / W_{0}} \tilde{\sigma}_{\epsilon, t}: \operatorname{Mod}_{f}(\mathcal{H} \rtimes \Gamma) \rightarrow \operatorname{Mod}_{f}\left(\mathcal{H}_{\epsilon} \rtimes \Gamma\right)
$$

is an additive functor which also has the properties described in Corollary 4.2.2. The functor $\tilde{\sigma}_{\epsilon}$ was already used in OpSo1, Theorem 1.7].

The image of $\tilde{\sigma}_{0}$ is contained in $\operatorname{Mod}_{T_{u n}}(\mathbb{C}[W])$, so this map is certainly not bijective, not even after passing to the associated Grothendieck groups of modules. Nevertheless, $\tilde{\sigma}_{0}$ clearly is related to the map $\zeta^{*}$ from Section 2.3 , in fact these maps agree on irreducible tempered $\mathcal{H} \rtimes \Gamma$-modules:

Lemma 4.2.3. Suppose that $\epsilon \in[-1,1], u c \in T_{u n} T_{r s}$ and $t \in T^{P(u)}$. Let $\Gamma_{u}^{\prime}$ be a subgroup of $W_{F_{u}, u}^{\prime}$ and $\pi \in \operatorname{Mod}_{f,\left(W\left(R_{u}\right) \rtimes \Gamma_{u}^{\prime}\right) u c}\left(\mathcal{H}\left(\tilde{\mathcal{R}}_{u}, q_{u}\right) \rtimes \Gamma_{u}^{\prime}\right)$.

(a) The following $\mathcal{H}_{\epsilon} \rtimes \Gamma$-representations are canonically isomorphic:

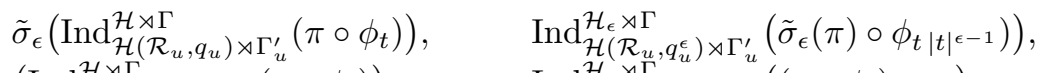

$$
\begin{aligned}
& \left(\operatorname{Ind}_{\mathcal{H}\left(\mathcal{R}_{u}, q_{u}\right) \rtimes \Gamma_{u}^{\prime}}^{\mathcal{H} \rtimes \Gamma}\left(\pi \circ \phi_{t}\right)\right) \circ \rho_{\epsilon}, \quad \operatorname{Ind}_{\mathcal{H}\left(\mathcal{R}_{u}, q_{u}^{\epsilon}\right) \rtimes \Gamma_{u}^{\prime}}^{\mathcal{H}_{\epsilon} \rtimes \Gamma}\left(\left(\pi \circ \phi_{t}\right) \circ \rho_{\epsilon}\right) \text {. }
\end{aligned}
$$


(b) $\tilde{\sigma}_{0}=\zeta^{*}$ on $\operatorname{Irr}(\mathcal{S} \rtimes \Gamma)$.

Proof. (a) By definition $\tilde{\sigma}_{\epsilon}$ is given by composition with $\tilde{\rho}_{\epsilon}$, and the difference between $\rho_{\epsilon}$ and $\tilde{\rho}_{\epsilon}$ is only an inner automorphism. Hence the map

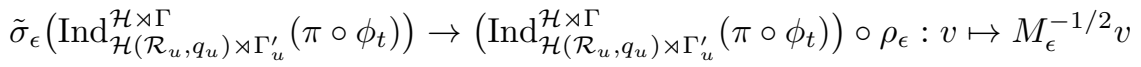

is an invertible intertwiner.

The two representations in the right-hand column are naturally isomorphic if and only if the $\mathcal{H}\left(\mathcal{R}_{u}, q_{u}^{\epsilon}\right) \rtimes \Gamma_{u}^{\prime}$-representations

$$
\tilde{\sigma}_{\epsilon}(\pi) \circ \phi_{t|t|^{\epsilon-1}} \text { and } \pi \circ \phi_{t} \circ \rho_{\epsilon}
$$

are so. Notice that $\phi_{t}$ and $\phi_{t|t|^{\epsilon-1}}$ are well-defined because $t \in T^{P(u)} \subseteq T^{W\left(R_{u}\right)}$. As we just showed, the left-hand side of (4.13) is naturally isomorphic to $\pi \circ \rho_{\epsilon} \circ \phi_{t|t| \epsilon-1}$. Applying $\sigma_{\epsilon}$ once with center $u c t$ and once with center $u c$ results in

$$
\sigma_{\epsilon, u c t}(u c t)=u t|t|^{-1} c^{\epsilon}|t|^{\epsilon}=\sigma_{\epsilon, u c}(u c) t|t|^{\epsilon-1} \text {. }
$$

This implies that $\rho_{\epsilon, u c} \circ \phi_{t|t| \epsilon-1}=\phi_{t} \circ \rho_{\epsilon, u c t}$, which shows that the representations (4.13) can indeed be identified.

Now we turn to the most difficult case, the two representations in the bottom row. In view of Theorem 2.1.2 $\mathrm{b}$

$$
\left(\operatorname{Ind}_{\mathcal{H}\left(\mathcal{R}_{u}, q_{u}\right) \rtimes \Gamma_{u}^{\prime}}^{\mathcal{H} \rtimes \Gamma}\left(\pi \circ \phi_{t}\right)\right) \circ \rho_{\epsilon} \quad \text { and } \quad \operatorname{Ind}_{\mathcal{H}\left(\mathcal{R}_{u}, q_{u}^{\epsilon}\right) \rtimes \Gamma_{u}^{\prime}}^{\mathcal{H}_{\epsilon} \rtimes \Gamma}\left(\pi \circ \phi_{t} \circ \rho_{\epsilon}\right)
$$

are isomorphic if only if the

$$
\mathcal{H}\left(\mathcal{R}_{u}, q_{u}^{\epsilon}\right)^{a n}\left(U_{\epsilon, u}\right) \rtimes W_{F_{u}, u}^{\prime} \text {-representation } \operatorname{Ind}_{\mathcal{H}\left(\mathcal{R}_{u}, q_{u}^{\epsilon}\right) \rtimes \Gamma_{u}^{\prime}}^{\mathcal{H}\left(\mathcal{R}_{u},{ }^{\epsilon}\right) \rtimes W_{\psi_{u}, u}^{\prime}}\left(\pi \circ \phi_{t} \circ \rho_{\epsilon}\right)
$$

corresponds to the

$1_{W_{u}^{\prime} u c}\left(\mathcal{H}\left(\mathcal{R}, q^{\epsilon}\right)^{a n}\left(U_{\epsilon}\right) \rtimes \Gamma\right) 1_{W_{u}^{\prime} u c^{-}}$-representation $1_{W_{u}^{\prime} u c}\left(\operatorname{Ind}_{\mathcal{H}\left(\mathcal{R}_{u}, q_{u}\right) \rtimes \Gamma_{u}^{\prime}}^{\mathcal{H} \rtimes \circ}\left(\pi \circ \phi_{t}\right)\right) \circ \rho_{\epsilon}$ via the isomorphism from Theorem 2.1.2. a. It is clear from the definition (4.3) that

$$
\operatorname{Ind}_{\mathcal{H}\left(\mathcal{R}_{u}, q_{u}^{\epsilon}\right) \rtimes \Gamma_{u}^{\prime}}^{\mathcal{H}\left(\mathcal{R}_{u}, u\right.}\left(\left(\pi \circ \phi_{t}\right) \circ \rho_{\epsilon}\right) \cong\left(\operatorname{Ind}_{\mathcal{H}\left(\mathcal{R}_{u}, q_{u}^{\epsilon}\right) \rtimes \Gamma_{u}^{\prime}}^{\mathcal{H}\left(\mathcal{R}_{u}, q_{u}^{\epsilon}\right) \rtimes W_{F_{u}, u}^{\prime}}\left(\pi \circ \phi_{t}\right)\right) \circ \rho_{\epsilon},
$$

so it suffices to show that the following diagram commutes:

$$
\begin{array}{ccc}
\mathcal{H}\left(\mathcal{R}_{u}, q_{u}^{\epsilon}\right)^{a n}\left(U_{\epsilon, u}\right) \rtimes W_{F_{u}, u}^{\prime} & \rightarrow & 1_{W_{u}^{\prime} u c}\left(\mathcal{H}\left(\mathcal{R}, q^{\epsilon}\right)^{a n}\left(U_{\epsilon}\right) \rtimes \Gamma\right) 1_{W_{u}^{\prime} u c} \\
\downarrow & \downarrow \rho_{\epsilon} & \\
& \rho_{\epsilon} \\
\mathcal{H}\left(\mathcal{R}_{u}, q_{u}\right)^{a n}\left(U_{u}\right) \rtimes W_{F_{u}, u}^{\prime} & \rightarrow & 1_{W_{u}^{\prime} u c}\left(\mathcal{H}(\mathcal{R}, q)^{a n}(U) \rtimes \Gamma\right) 1_{W_{u}^{\prime} u c} .
\end{array}
$$

For elements of $\mathcal{H}\left(\mathcal{R}_{u}, q_{u}^{\epsilon}\right)^{a n}\left(U_{\epsilon, u}\right)$ this is easy, since the effect of the vertical arrows is only extension of functions from $U_{\epsilon, u}$ (resp. $U_{u}$ ) to $U_{\epsilon}$ (resp. $U$ ) by 0 . For elements of $W_{F_{u}, u}^{\prime}$ the commutativity follows from (2.2) and (4.3).

(b) By Corollary 2.1.5 b every irreducible tempered $\mathcal{H} \rtimes \Gamma$-representation $\pi$ is of the form $\operatorname{Ind}_{\mathcal{H}\left(\mathcal{R} \mathcal{R}_{u}, q_{u}\right) \rtimes W_{F_{u}, u}^{\prime}}^{\mathcal{H}}\left(\tilde{\pi} \circ \Phi_{u}\right)$ for some $\tilde{\pi} \in \operatorname{Irr}_{0}\left(\mathcal{H}\left(\mathcal{R}_{u}, q_{u}\right)^{a n}\left(U_{u}\right) \rtimes W_{F_{u}, u}^{\prime}\right)$. By Theorem 2.3.1 d

$$
\zeta^{*}(\pi)=\operatorname{Ind}_{X \rtimes W_{u}^{\prime}}^{X \rtimes W_{u}^{\prime}}\left(\left.\mathbb{C}_{u} \otimes \tilde{\pi}\right|_{W_{u}^{\prime}}\right) .
$$

Using (4.7) we can rewrite

$$
\left.\mathbb{C}_{u} \otimes \tilde{\pi}\right|_{W_{u}^{\prime}}=\left.\mathbb{C}_{u} \otimes\left(\tilde{\pi} \circ m_{0}\right)\right|_{W_{u}^{\prime}}=\tilde{\pi} \circ m_{0} \circ \Phi_{u, 0}=\tilde{\pi} \circ \Phi_{u} \circ \rho_{0} \cong \tilde{\pi} \circ \Phi_{u} \circ \tilde{\rho}_{0}=\tilde{\sigma}_{0}\left(\tilde{\pi} \circ \Phi_{u}\right) \text {. }
$$

Now we can apply part (a):

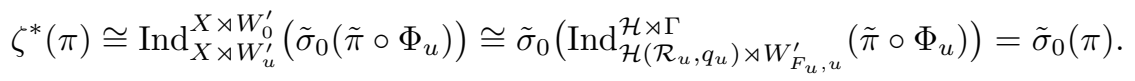


4.3. Scaling intertwining operators. We will show that the scaling maps $\tilde{\sigma}_{\epsilon}$ give rise to scaled versions of the intertwining operators $\pi^{\Gamma}(g, \xi)$. We will use this to study the behaviour of the components of the Fourier transform of $\mathcal{S} \rtimes \Gamma$ under scaling of $q$.

As we remarked at the start of Section 4.1, the results of that section can easily be extended to $\mathcal{H} \rtimes \Gamma$, and we will use that generality here. Recall that the groupoid $\mathcal{G}$ from (3.13) includes $\Gamma$ and is defined independently of $q$. Let us realize the representation

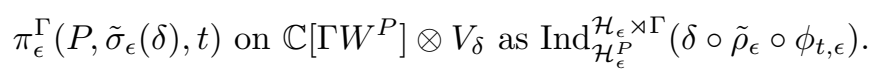

For all $\epsilon \in[-1,1]$ we obtain algebra homomorphisms

$$
\begin{aligned}
& \mathcal{F}_{\epsilon}: \mathcal{H}\left(\mathcal{R}, q^{\epsilon}\right) \rtimes \Gamma \rightarrow \bigoplus_{P, \delta} \mathcal{O}\left(T^{P}\right) \otimes \operatorname{End}_{\mathbb{C}}\left(\mathbb{C}\left[\Gamma W^{P}\right] \otimes V_{\delta}\right), \\
& \mathcal{F}_{\epsilon}(h)(P, \delta, t)=\pi_{\epsilon}^{\Gamma}\left(P, \tilde{\sigma}_{\epsilon}(\delta), t, h\right) .
\end{aligned}
$$

Rational intertwining operators $\pi_{\epsilon}^{\Gamma}\left(g, P, \delta^{\prime}, t\right)$ can be defined as in (3.12) for all $\mathcal{H}_{\epsilon^{-}}$ representations of the form $\pi_{\epsilon}^{\Gamma}\left(P, \delta^{\prime}, t\right)$, where $\delta^{\prime}$ is irreducible but not necessarily discrete series. In particular, for $\epsilon \neq 0$ the $(P, \delta)$-component of the image of $\mathcal{F}_{\epsilon}$ is invariant under an action of the group

$$
\mathcal{G}_{P, \tilde{\sigma}_{\epsilon}(\delta)}:=\left\{g \in \mathcal{G}: g(P)=P, \tilde{\sigma}_{\epsilon}(\delta) \circ \psi_{g}^{-1} \cong \tilde{\sigma}_{\epsilon}(\delta)\right\}
$$

via such intertwiners. As in (3.16), the action is not on polynomial but on rational sections.

Proposition 4.3.1. Let $\epsilon \in[-1,1] \backslash\{0\}$, let $g \in \mathcal{G}$ with $g(P) \subset F_{0}$ and let $\delta^{\prime}$ be a discrete series representation of $\mathcal{H}_{g(P)}$ that is equivalent to $\delta \circ \psi_{g}^{-1}$.

(a) The $\mathcal{H}_{g(P), \epsilon}$-representations $\tilde{\sigma}_{\epsilon}\left(\delta^{\prime}\right)$ and $\tilde{\sigma}_{\epsilon}(\delta) \circ \psi_{g, \epsilon}^{-1}$ are unitarily equivalent.

(b) $\mathcal{G}_{P, \tilde{\sigma}_{\epsilon}(\delta)}=\mathcal{G}_{P, \delta}$ and $\mathcal{G}_{P, \tilde{\sigma}_{\epsilon}(\delta), t}=\mathcal{G}_{P, \delta, t}$ for all $t \in T^{P}$.

(c) The intertwiners $\pi_{\epsilon}^{\Gamma}\left(g, P, \tilde{\sigma}_{\epsilon}(\delta), t\right) \in \operatorname{Hom}_{\mathbb{C}}\left(\mathbb{C}\left[\Gamma W^{P}\right] \otimes V_{\delta}, \mathbb{C}\left[\Gamma W^{g(P)}\right] \otimes V_{\delta^{\prime}}\right)$ depend rationally on $t \in T^{P}$ and analytically on $\epsilon$, whenever they are regular.

(d) For $t \in T_{u n}^{P}$ the $\pi_{\epsilon}^{\Gamma}\left(g, P, \tilde{\sigma}_{\epsilon}(\delta), t\right)$ are regular and unitary, and $\pi_{0}^{\Gamma}(g, P$, $\left.\tilde{\sigma}_{0}(\delta), t\right):=\lim _{\epsilon \rightarrow 0} \pi_{\epsilon}^{\Gamma}\left(g, P, \tilde{\sigma}_{\epsilon}(\delta), t\right)$ exists.

Proof. (a) First we show that

$$
\psi_{g} \circ \rho_{\epsilon}=\rho_{\epsilon} \circ \psi_{g, \epsilon} .
$$

Write $g=\gamma^{-1} u$ with $u \in K_{P}$ and $\gamma \in W_{0} \rtimes \Gamma$. The automorphism $\psi_{u}$ from (1.21) reflects the translation of $T^{P}$ by $u \in T_{u n}^{P}$ : that changes $U$ to $u U$, but apart from that it commutes with $\sigma_{\epsilon}$. Hence $\psi_{u} \circ \rho_{\epsilon}=\rho_{\epsilon} \circ \psi_{u, \epsilon}$.

The isomorphism $\psi_{\gamma}: \mathcal{H}^{P} \rightarrow \mathcal{H}^{\gamma(P)}$ from (1.17) is more difficult to deal with, because it acts nontrivially on the $N_{w}$ with $w \in W_{P}$. However, by Proposition 1.5.1, d this is the restriction to $\mathcal{H}^{P}$ of the automorphism

$$
\psi_{\gamma}: h \mapsto \imath_{\gamma}^{0} h \imath_{\gamma^{-1}}^{0} \text { of the algebra }\left(\mathbb{C}\left(T / W_{0}\right) \otimes_{Z(\mathcal{H})} \mathcal{H}\right) \rtimes \Gamma .
$$

Similarly, $\psi_{\gamma, \epsilon}(h)=\imath_{\gamma, \epsilon}^{0} h \imath_{\gamma^{-1}, \epsilon}^{0}$. From these formula it is clear that $\psi_{\gamma} \circ \rho_{\epsilon}=\rho_{\epsilon} \circ \psi_{\gamma, \epsilon}$ on $\mathcal{H}_{\epsilon}^{m e}\left(U_{\epsilon}\right)$. This establishes (4.15).

Let $I_{\delta}^{g}: V_{\delta} \rightarrow V_{\delta^{\prime}}$ be as in (3.11). We claim that

$$
v \mapsto I_{\delta}^{g}\left(\delta\left(\psi_{g}^{-1}\left(M_{\epsilon}^{1 / 2}\right) M_{\epsilon}^{-1 / 2}\right) v\right)
$$


is an intertwiner between $\tilde{\sigma}_{\epsilon}(\delta) \circ \psi_{g, \epsilon}^{-1}$ and $\tilde{\sigma}_{\epsilon}\left(\delta^{\prime}\right)$. Indeed, for $v \in V_{\delta}$ and $h \in$ $\mathcal{H}_{\epsilon}^{a n}\left(U_{\epsilon}\right)$ :

$$
\begin{aligned}
I_{\delta}^{g}( & \left.\delta\left(\psi_{g}^{-1}\left(M_{\epsilon}^{1 / 2}\right) M_{\epsilon}^{-1 / 2}\right) \tilde{\sigma}_{\epsilon}(\delta) \circ \psi_{g, \epsilon}(h) v\right) \\
& =I_{\delta}^{g}\left(\delta\left(\psi_{g}^{-1}\left(M_{\epsilon}^{1 / 2} M_{\epsilon}^{-1 / 2}\right) \delta\left(M_{\epsilon}^{1 / 2} \rho_{\epsilon}\left(\psi_{g, \epsilon}^{-1} h\right) M_{\epsilon}^{-1 / 2}\right) v\right)\right. \\
& =I_{\delta}^{g}\left(\delta \circ \psi_{g}^{-1}\left(M_{\epsilon}^{1 / 2} \rho_{\epsilon}(h) M_{\epsilon}^{-1 / 2}\right) \delta\left(\psi_{g}^{-1}\left(M_{\epsilon}^{1 / 2}\right) M_{\epsilon}^{-1 / 2}\right) v\right) \\
& =\delta^{\prime}\left(M_{\epsilon}^{1 / 2} \rho_{\epsilon}(h) M_{\epsilon}^{-1 / 2}\right) I_{\delta}^{g}\left(\delta\left(\psi_{g}^{-1}\left(M_{\epsilon}^{1 / 2}\right) M_{\epsilon}^{-1 / 2}\right) v\right) \\
& =\tilde{\sigma}_{\epsilon}\left(\delta^{\prime}\right)(h) I_{\delta}^{g}\left(\delta\left(\psi_{g}^{-1}\left(M_{\epsilon}^{1 / 2}\right) M_{\epsilon}^{-1 / 2}\right) v\right) .
\end{aligned}
$$

Obviously (4.16) is invertible, so it is an equivalence between the irreducible representations $\tilde{\sigma}_{\epsilon}(\delta) \circ \psi_{g, \epsilon}^{-1}$ and $\tilde{\sigma}_{\epsilon}\left(\delta^{\prime}\right)$. Since both are unitary, there exists a unitary intertwiner between, which by the irreducibility must be a scalar multiple of (4.16). We define $I_{\delta, \epsilon}^{g}$ as the unique positive multiple of (4.16) that is unitary.

(b) By part (a),

$$
\begin{aligned}
g\left(P, \tilde{\sigma}_{\epsilon}(\delta), t\right)=\left(g(P), \tilde{\sigma}_{\epsilon}(\delta) \circ \psi_{g, \epsilon}^{-1}, g(t)\right) & \cong\left(g(P), \tilde{\sigma}_{\epsilon}\left(\delta^{\prime}\right), g(t)\right) \\
& \cong\left(g(P), \tilde{\sigma}_{\epsilon}\left(\delta \circ \psi_{g}^{-1}\right), g(t)\right),
\end{aligned}
$$

so the stabilizer of $\left(P, \tilde{\sigma}_{\epsilon}(\delta), t\right)$ does not depend on $\epsilon \in[-1,1]$.

(c) By Theorem 4.2.1] $I_{\delta, \epsilon}^{g}$ depends analytically on $\epsilon \in[-1,1]$. By definition $\imath_{\gamma}^{0}$ is rational in $t \in T$ and analytic in $\epsilon$, away from the poles. By definition (3.12)

$$
\pi_{\epsilon}^{\Gamma}\left(g, P, \tilde{\sigma}_{\epsilon}(\delta), t\right)\left(h \otimes_{\mathcal{H}_{\epsilon}^{P}} v\right)=h r_{\gamma}^{0} \otimes_{\mathcal{H}_{\epsilon}^{g(P)}} I_{\delta, \epsilon}^{g}(v),
$$

so $\pi_{\epsilon}^{\Gamma}\left(g, P, \tilde{\sigma}_{\epsilon}(\delta), t\right)$ has the required properties.

(d) All possible singularities of the intertwining operators come from poles and zeros of the $c$-functions from (1.6). By Theorem 3.1.5 $\mathrm{c} \pi_{\epsilon}^{\Gamma}\left(g, P, \tilde{\sigma}_{\epsilon}(\delta), t\right)$ is unitary for all $t \in T_{u n}^{P}$ and $\epsilon \in(0,1]$. In particular, all the singularities on this domain are removable. On the other hand, the explicit formula for $c_{\alpha}$ shows that the singularities for $q^{\epsilon}(\epsilon \neq 0)$ and $t \in T_{u n}$ are the same as those for $q$ and $t \in T_{u n}$. Therefore $\pi_{\epsilon}^{\Gamma}\left(g, P, \tilde{\sigma}_{\epsilon}(\delta), t\right)$ is also regular for $\epsilon \in[-1,0)$ and $t \in T_{u n}^{P}$.

Since these linear maps are analytic in $\epsilon \in[-1,1] \backslash\{0\}$ and unitary for $\epsilon>0$, they are also unitary for $\epsilon<0$. Hence all the matrix coefficients of $\pi_{\epsilon}^{\Gamma}\left(g, P, \tilde{\sigma}_{\epsilon}(\delta), t\right)$ are uniformly bounded on $T_{u n}^{P} \times[-1,1] \backslash\{0\}$, which implies that every possible singularity at $\epsilon=0$ is removable. In particular, $\lim _{\epsilon \rightarrow 0} \pi_{\epsilon}^{\Gamma}\left(g, P, \tilde{\sigma}_{\epsilon}(\delta), t\right)$ exists.

We fix a discrete series representation $\delta$ of $\mathcal{H}_{P}$ and we abbreviate

$$
A_{P, \delta}:=C^{\infty}\left(T_{u n}^{P}\right) \otimes_{\mathbb{C}}\left(\mathbb{C}\left[\Gamma W^{P}\right] \otimes V_{\delta}\right) .
$$

Proposition 4.3.1 says among others that for $g \in \mathcal{G}_{P, \delta}$,

$$
[-1,1] \rightarrow A_{P, \delta}^{\times}: \epsilon \mapsto \pi_{\epsilon}^{\Gamma}\left(g, P, \tilde{\sigma}_{\epsilon}(\delta), \cdot\right),
$$

is an analytic map. The group $\mathcal{G}_{P, \tilde{\sigma}_{\epsilon}(\delta)}=\mathcal{G}_{P, \delta}$ acts on $A_{P, \delta}$ by

$$
\left(g \cdot{ }_{\epsilon} f\right)(t)=\pi_{\epsilon}^{\Gamma}\left(g, P, \tilde{\sigma}_{\epsilon}(\delta), g^{-1} t\right) f\left(g^{-1} t\right) \pi_{\epsilon}^{\Gamma}\left(g, P, \tilde{\sigma}_{\epsilon}(\delta), g^{-1} t\right)^{-1} .
$$

By construction the $\delta$-component of the image of $\mathcal{F}_{\epsilon}$ consists of $\mathcal{G}_{P, \tilde{\sigma}_{\epsilon}(\delta)}$-invariant sections for $\epsilon \neq 0$, and by Proposition 4.3.1 this also goes for $\epsilon=0$. We intend to show that the algebras $A_{P, \delta}^{\mathcal{G}_{P, \tilde{\sigma}_{\epsilon}(\delta)}}$ for $\epsilon \in[-1,1]$ are all isomorphic. (Although 
$\mathcal{G}_{P, \tilde{\sigma}_{\epsilon}(\delta)}=\mathcal{G}_{P, \delta}$ we prefer the longer notation here, because it indicates which action on $A_{P, \delta}$ we consider.) We must be careful when taking invariants, because

$$
\mathcal{G}_{P, \delta} \rightarrow A_{P, \delta}^{\times}: g \mapsto \pi_{\epsilon}^{\Gamma}\left(g, P, \tilde{\sigma}_{\epsilon}(\delta), \cdot\right)
$$

is not necessarily a group homomorphism. However, the lack of multiplicativity is small, it is only caused by the freedom in the choice of a scalar in (3.11). In other words, (4.18) defines a projective representation of $\mathcal{G}_{P, \delta}$ on $A_{P, \delta}$. Recall CuRe, Section 53] that the Schur multiplier $\mathcal{G}_{P, \delta}^{*}$ is a finite central extension of $\mathcal{G}_{P, \delta}$, with the property that every projective representation of $\mathcal{G}_{P, \delta}$ lifts to a unique linear representation of $\mathcal{G}_{P, \delta}^{*}$. This means that for every lift $g^{*} \in \mathcal{G}_{P, \delta}^{*}$ of $g \in \mathcal{G}_{P, \delta}$ there is a unique scalar multiple $\pi_{\epsilon}^{\Gamma}\left(g^{*}, P, \tilde{\sigma}_{\epsilon}(\delta), \cdot\right)$ of $\pi_{\epsilon}^{\Gamma}\left(g, P, \tilde{\sigma}_{\epsilon}(\delta), \cdot\right)$ such that

$$
\mathcal{G}_{P, \delta}^{*} \rightarrow A_{P, \delta}^{\times}: g^{*} \mapsto \pi_{\epsilon}^{\Gamma}\left(g^{*}, P, \tilde{\sigma}_{\epsilon}(\delta), \cdot\right)
$$

becomes multiplicative. Since $\pi_{\epsilon}^{\Gamma}\left(g, P, \tilde{\sigma}_{\epsilon}(\delta), \cdot\right)$ is unitary, so is $\pi_{\epsilon}^{\Gamma}\left(g^{*}, P, \tilde{\sigma}_{\epsilon}(\delta), \cdot\right)$. Notice that $\mathcal{G}_{P, \delta}$ and $\mathcal{G}_{P, \delta}^{*}$ fix the same elements of $A_{P, \delta}$, because the action (4.17) is defined via conjugation with $\pi_{\epsilon}^{\Gamma}\left(g, P, \tilde{\sigma}_{\epsilon}(\delta), \cdot\right)$. According to [CuRe, Section 53] the way to lift (4.18) from $\mathcal{G}_{P, \delta}$ to $\mathcal{G}_{P, \delta}^{*}$ is completely determined by the cohomology class of the 2-cocycle

$$
\mathcal{G}_{P, \delta} \times \mathcal{G}_{P, \delta} \rightarrow \mathbb{C}^{\times}:\left(g_{1}, g_{2}\right) \mapsto I_{\delta, \epsilon}^{g_{1}} I_{\delta, \epsilon}^{g_{2}} I_{\delta, \epsilon}^{g_{2}^{-1} g_{1}^{-1}} .
$$

This cocycle depends analytically on $\epsilon$ and $\mathcal{G}_{P, \delta}$ is a finite group, so the class of (4.19) in $H^{2}\left(\mathcal{G}_{P, \delta}, \mathbb{C}\right)$ does not depend on $\epsilon$. (In most cases this cohomology class is trivial, but examples are known in which it is nontrivial, see DeOp2, Section 6.2].) Hence the ratio between $\pi_{\epsilon}^{\Gamma}\left(g^{*}, P, \tilde{\sigma}_{\epsilon}(\delta), \cdot\right)$ and $\pi_{\epsilon}^{\Gamma}\left(g, P, \tilde{\sigma}_{\epsilon}(\delta), \cdot\right)$ also depends analytically on $\epsilon$.

For $g^{*} \in \mathcal{G}_{P, \delta}^{*}$ we define $\lambda_{g^{*}}: T^{P} \rightarrow T^{P}$ by $\lambda_{g^{*}}(t)=g(t)$. In the remainder of this section we will work mostly with $\mathcal{G}_{P, \delta}^{*}$, and to simplify the notation we will denote its typical elements by $g$ instead of $g^{*}$. For $g \in \mathcal{G}_{P, \delta}^{*}$ and $t \in T_{u n}^{P}$ we write

$$
u_{g, \epsilon}(t):=\pi_{\epsilon}^{\Gamma}\left(g, \lambda_{g}^{-1}(t)\right),
$$

so that the multiplicativity translates into

$$
u_{g g^{\prime}, \epsilon}=u_{g, \epsilon}\left(u_{g^{\prime}, \epsilon} \circ \lambda_{g}^{-1}\right) .
$$

From the above we know that $u_{g, \epsilon} \in A_{P, \delta}$ is unitary and analytic in $\epsilon$. These elements can be used to identify $A_{P, \delta}^{\mathcal{G}_{P, \delta} \tilde{\epsilon}_{\epsilon}(\delta)}$ with a corner in a larger algebra. Consider the crossed product $A_{P, \delta} \rtimes_{\lambda} \mathcal{G}_{P, \delta}^{*}$, where the action of $\mathcal{G}_{P, \delta}^{*}$ on $A_{P, \delta}$ comes only from the action on $C\left(T_{u n}^{P}\right)$ induced by the $\lambda_{g}$. In particular, this action is independent of $\epsilon$. On $A_{P, \delta} \rtimes_{\lambda} \mathcal{G}_{P, \delta}^{*}$ we can define actions of $\mathcal{G}_{P, \delta}^{*}$ by

$$
g \cdot{ }_{\epsilon} a=u_{g, \epsilon} g a g^{-1} u_{g, \epsilon}^{-1} .
$$

For $a \in A_{P, \delta}$ this recovers the action (4.17). An advantage of introducing the Schur multiplier is that, by (4.20), $g \mapsto u_{g, \epsilon} g$ is a homomorphism from $\mathcal{G}_{P, \delta}^{*}$ to the unitary group of $A_{P, \delta} \rtimes_{\lambda} \mathcal{G}_{P, \delta}^{*}$. Hence

$$
[-1,1] \rightarrow A_{P, \delta} \rtimes_{\lambda} \mathcal{G}_{P, \delta}^{*}: \epsilon \mapsto p_{\delta, \epsilon}:=\left|\mathcal{G}_{P, \delta}^{*}\right|^{-1} \sum_{g \in \mathcal{G}_{P, \delta}^{*}} u_{g, \epsilon} g
$$


is a family of projections, depending analytically on $\epsilon$. As was first observed in Ros, and worked out in [Sol3, Lemma A.2],

$$
A_{P, \delta}^{\mathcal{G}_{P, \tilde{\sigma}_{\epsilon}(\delta)}} \rightarrow p_{\delta, \epsilon}\left(A_{P, \delta} \rtimes_{\lambda} \mathcal{G}_{P, \delta}^{*}\right) p_{\delta, \epsilon}: a \mapsto p_{\delta, \epsilon} a p_{\delta, \epsilon}
$$

is an isomorphism of Fréchet *-algebras. Its inverse is the map

$$
\sum_{g \in \mathcal{G}_{P, \delta}^{*}} a_{g} g \mapsto\left|\mathcal{G}_{P, \delta}^{*}\right| a_{e} .
$$

Lemma 4.3.2. The Fréchet ${ }^{*}$-algebras $A_{P, \delta}^{\mathcal{G}_{P, \tilde{\sigma}_{\epsilon}(\delta)}} \cong p_{\delta, \epsilon}\left(A_{P, \delta} \rtimes_{\lambda} \mathcal{G}_{P, \delta}^{*}\right) p_{\delta, \epsilon}$ are all

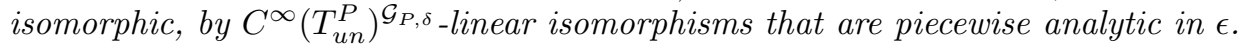

Proof. According to [Phi, Lemma 1.15] the projections $p_{\delta}\left(u_{\epsilon}\right)$ are all conjugate, by elements depending continuously on $\epsilon$. That already proves that all the Fréchet algebras $A_{P, \delta}^{\mathcal{G}_{P, \tilde{\sigma}_{\epsilon}(\delta)}}$ are isomorphic. Since $C^{\infty}\left(T_{u n}^{P}\right)^{\mathcal{G}_{P, \delta}}$ is the center of both $A_{P, \delta}^{\mathcal{G}_{P, \tilde{\sigma}_{\epsilon}(\delta)}}$ and $p_{\delta, \epsilon}\left(A_{P, \delta} \rtimes_{\lambda} \mathcal{G}_{P, \delta}^{*}\right) p_{\delta, \epsilon}$, these isomorphisms are $C^{\infty}\left(T_{u n}^{P}\right)^{\mathcal{G}_{P, \delta} \text {-linear. }}$

To show that the isomorphisms can be made piecewise analytic we construct the conjugating elements explicitly, using the recipe of [Bla, Proposition 4.32]. For $\epsilon, \epsilon^{\prime} \in[-1,1]$ consider

$$
z\left(\delta, \epsilon, \epsilon^{\prime}\right):=\left(2 p_{\delta, \epsilon^{\prime}}-1\right)\left(2 p_{\delta, \epsilon}-1\right)+1 \in A_{P, \delta} \rtimes_{\lambda} \mathcal{G}_{P, \delta}^{*} .
$$

Clearly, this is analytic in $\epsilon$ and $\epsilon^{\prime}$ and

$$
p_{\delta, \epsilon^{\prime}} z\left(\delta, \epsilon, \epsilon^{\prime}\right)=2 p_{\delta, \epsilon^{\prime}} p_{\delta, \epsilon}=z\left(\delta, \epsilon, \epsilon^{\prime}\right) p_{\delta, \epsilon} .
$$

The enveloping $C^{*}$-algebra of $A_{P, \delta} \rtimes_{\lambda} \mathcal{G}_{P, \delta}^{*}$ is

$$
C_{\delta}:=\operatorname{End}_{\mathbb{C}}\left(\mathbb{C}\left[\Gamma W^{P}\right] \otimes V_{\delta}\right) \otimes C\left(T_{u n}^{P}\right) \rtimes_{\lambda} \mathcal{G}_{P, \delta}^{*} .
$$

Let $\|\cdot\|$ be its norm and suppose that $\left\|p_{\delta}\left(u_{\epsilon}\right)-p_{\delta}\left(u_{\epsilon}^{\prime}\right)\right\|<1$. Then

$$
\begin{aligned}
\left\|z\left(\delta, \epsilon, \epsilon^{\prime}\right)-2\right\| & =\left\|4 p_{\delta, \epsilon^{\prime}} p_{\delta, \epsilon}-2 p_{\delta, \epsilon}-2 p_{\delta, \epsilon^{\prime}}\right\| \\
& =\left\|-2\left(p_{\delta, \epsilon}-p_{\delta, \epsilon^{\prime}}\right)^{2}\right\| \\
& \leq 2\left\|p_{\delta, \epsilon}-p_{\delta, \epsilon^{\prime}}\right\|^{2}<2
\end{aligned}
$$

so $z\left(\delta, \epsilon, \epsilon^{\prime}\right)$ is invertible in $C_{\delta}$. But $A_{P, \delta} \rtimes_{\lambda} \mathcal{G}_{P, \delta}^{*}$ is closed under the holomorphic functional calculus of $C_{\delta}$, so $z\left(\delta, \epsilon, \epsilon^{\prime}\right)$ is also invertible in this Fréchet algebra. Moreover, because the Fréchet topology on $A_{P, \delta} \rtimes_{\lambda} \mathcal{G}_{P, \delta}^{*}$ is finer than the induced topology from $C_{\delta}$, there exists an open interval $I_{\epsilon}$ containing $\epsilon$ such that $z\left(\delta, \epsilon, \epsilon^{\prime}\right)$ is invertible for all $\epsilon^{\prime} \in I_{\epsilon}$. For such $\epsilon, \epsilon^{\prime}$ we construct the unitary element

$$
u\left(\delta, \epsilon, \epsilon^{\prime}\right):=z\left(\delta, \epsilon, \epsilon^{\prime}\right)\left|z\left(\delta, \epsilon, \epsilon^{\prime}\right)\right|^{-1} \in A_{P, \delta} \rtimes_{\lambda} \mathcal{G}_{P, \delta}^{*} .
$$

By construction, the map

$$
p_{\delta, \epsilon}\left(A_{P, \delta} \rtimes_{\lambda} \mathcal{G}_{P, \delta}^{*}\right) p_{\delta, \epsilon} \rightarrow p_{\delta, \epsilon^{\prime}}\left(A_{P, \delta} \rtimes_{\lambda} \mathcal{G}_{P, \delta}^{*}\right) p_{\delta, \epsilon^{\prime}}: x \mapsto u\left(\delta, \epsilon, \epsilon^{\prime}\right) x u\left(\delta, \epsilon, \epsilon^{\prime}\right)^{-1}
$$

is an isomorphism of Fréchet *-algebras. Notice that (4.24) also defines an isomorphism between the respective $C^{*}$-completions.

Let us pick a finite cover $\left\{I_{\epsilon_{i}}\right\}_{i=1}^{m}$ of $[-1,1]$. Then for every $\epsilon, \epsilon^{\prime} \in[-1,1]$ an isomorphism between $p_{\delta, \epsilon}\left(A_{P, \delta} \rtimes_{\lambda} \mathcal{G}_{P, \delta}^{*}\right) p_{\delta, \epsilon}$ and $p_{\delta, \epsilon^{\prime}}\left(A_{P, \delta} \rtimes_{\lambda} \mathcal{G}_{P, \delta}^{*}\right) p_{\delta, \epsilon^{\prime}}$ can be obtained by composing at most $m$ isomorphisms of the form (4.24). 
4.4. Scaling Schwartz algebras. It follows from Corollary 4.2 .2 that, for $\epsilon \in$ $(0,1]$, the functor $\tilde{\sigma}_{\epsilon}$ is an equivalence between the categories of finite dimensional tempered modules of $\mathcal{H}$ and of $\mathcal{H}_{\epsilon}=\mathcal{H}\left(\mathcal{R}, q^{\epsilon}\right)$. We will combine this with the explicit description of the Fourier transform of $\mathcal{S}(\mathcal{R}, q)$ from Theorem 3.2.2 to construct "scaling isomorphisms"

$$
\zeta_{\epsilon}: \mathcal{S}\left(\mathcal{R}, q^{\epsilon}\right) \rtimes \Gamma \rightarrow \mathcal{S}(\mathcal{R}, q) \rtimes \Gamma .
$$

These algebra homomorphisms depend continuously on $\epsilon$ and they turn out to have a well-defined limit:

$$
\zeta_{0}: \mathcal{S}(W) \rtimes \Gamma=\mathcal{S}\left(\mathcal{R}, q^{0}\right) \rtimes \Gamma \rightarrow \mathcal{S}(\mathcal{R}, q) \rtimes \Gamma .
$$

Although $\zeta_{0}$ is no longer surjective, it provides the connection between the representation theories of $\mathcal{S}(\mathcal{R}, q)$ and $\mathcal{S}(W)$ that we already discussed in Section [2.3. Recall from (3.26) that $\Delta$ is a set of representatives for $\mathcal{G}$-association classes of discrete series representations of parabolic subalgebras of $\mathcal{H}$, and that

$$
\mathcal{F}: \mathcal{S}(\mathcal{R}, q) \rtimes \Gamma \rightarrow \bigoplus_{(P, \delta) \in \Delta}\left(C^{\infty}\left(T_{u n}^{P}\right) \otimes \operatorname{End}_{\mathbb{C}}\left(\mathbb{C}\left[\Gamma W^{P}\right] \otimes V_{\delta}\right)\right)^{\mathcal{G}_{P, \delta}}
$$

is an isomorphism of Fréchet *-algebras. Together with (4.14) and Lemma 4.3.2, this implies the existence of a continuous family of algebra homomorphisms, with some nice properties:

Proposition 4.4.1. There exists a collection of injective *-homomorphisms

$$
\zeta_{\epsilon}: \mathcal{H}\left(\mathcal{R}, q^{\epsilon}\right) \rtimes \Gamma \rightarrow \mathcal{S}(\mathcal{R}, q) \rtimes \Gamma \quad \epsilon \in[-1,1],
$$

such that:

(a) For $\epsilon<0$ (respectively $\epsilon>0$ ) the map $\pi \mapsto \pi \circ \zeta_{\epsilon}$ is an equivalence between $\operatorname{Mod}_{f}(\mathcal{S}(\mathcal{R}, q) \rtimes \Gamma)$ and the category of finite dimensional anti-tempered (respectively tempered) $\mathcal{H}\left(\mathcal{R}, q^{\epsilon}\right) \rtimes \Gamma$-modules.

(b) $\zeta_{1}: \mathcal{H} \rtimes \Gamma \rightarrow \mathcal{S} \rtimes \Gamma$ is the canonical embedding.

(c) $\zeta_{\epsilon}\left(N_{w}\right)=N_{w}$ for all $w \in Z(W)$.

(d) For all $w \in W$ the map

$$
[-1,1] \rightarrow \mathcal{S}(\mathcal{R}, q) \rtimes \Gamma: \epsilon \mapsto \zeta_{\epsilon}\left(N_{w}\right)
$$

is piecewise analytic, and in particular analytic at 0 .

(e) For all $\pi \in \operatorname{Mod}_{f}(\mathcal{S}(\mathcal{R}, q) \rtimes \Gamma)$ the representations $\pi \circ \zeta_{\epsilon}$ and $\tilde{\sigma}_{\epsilon}(\pi)$ are equivalent. In particular, $\pi^{\Gamma}(P, \delta, t) \circ \zeta_{\epsilon} \cong \pi_{\epsilon}^{\Gamma}\left(P, \tilde{\sigma}_{\epsilon}(\delta), t\right)$ for all $(P, \delta, t) \in$ $\Xi_{\text {un }}$.

(f) $\zeta_{\epsilon}$ preserves the trace $\tau$.

Proof. Let $\zeta_{P, \delta, \epsilon}: A_{P, \delta}^{\mathcal{G}_{P, \tilde{\sigma}_{\epsilon}(\delta)}} \rightarrow A_{P, \delta}^{\mathcal{G}_{P, \delta}}$ be the isomorphism from Lemma 4.3.2, We already observed that $\delta$-component of the image of $\mathcal{F}_{\epsilon}$ is invariant under $\mathcal{G}_{P, \tilde{\sigma}_{\epsilon}(\delta)}$, so we can define

$$
\zeta_{\epsilon}:=\mathcal{F}^{-1} \circ\left(\bigoplus_{(P, \delta) \in \Delta} \zeta_{P, \delta, \epsilon}\right) \circ \mathcal{F}_{\epsilon} .
$$

Now (b) holds by construction and (c) follows from the $C^{\infty}\left(T_{u n}^{P}\right)^{\mathcal{G}_{P, \delta}}$-linearity in Lemma 4.3.2. For (d) we use Theorem 4.2.1 and Lemma 4.3.2, From the last lines of the proof of Lemma 4.3.2 we see how we can arrange that $\zeta_{\epsilon}$ is analytic at 0 : it suffices to take $\epsilon_{i}=0$ and to use the elements $u\left(\delta, 0, \epsilon^{\prime}\right)$ for $\epsilon^{\prime}$ in a neighborhood of $\epsilon=0$. 
Any finite dimensional module decomposes canonically as a direct sum of parts corresponding to different central characters. Hence in (e) it suffices to consider $(\pi, V) \in \operatorname{Mod}_{f, \mathcal{G}(P, \delta, t)}(\mathcal{S}(\mathcal{R}, q) \rtimes \Gamma)$. That is, $\pi: \mathcal{S}(\mathcal{R}, q) \rtimes \Gamma \rightarrow \operatorname{End}_{\mathbb{C}}(V)$ factors via

$$
\operatorname{ev}_{P, \delta, t}: \mathcal{S}(\mathcal{R}, q) \rtimes \Gamma \rightarrow \operatorname{End}_{\mathbb{C}}\left(\mathbb{C}\left[\Gamma W^{P}\right] \otimes V_{\delta}\right), h \mapsto \pi^{\Gamma}(P, \delta, t, h) .
$$

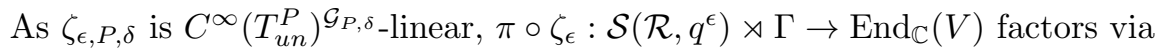

$$
\operatorname{ev}_{P, \tilde{\sigma}_{\epsilon}(\delta), t}: \mathcal{S}\left(\mathcal{R}, q^{\epsilon}\right) \rtimes \Gamma \rightarrow \operatorname{End}_{\mathbb{C}}\left(\mathbb{C}\left[\Gamma W^{P}\right] \otimes V_{\delta}\right) .
$$

Moreover, by Lemma 4.3 .2 the finite dimensional $C^{*}$-algebras $\operatorname{ev}_{P, \delta, t}(\mathcal{S}(\mathcal{R}, q) \rtimes \Gamma)$ and $\operatorname{ev}_{P, \tilde{\sigma}_{\epsilon}(\delta), t}\left(\mathcal{S}\left(\mathcal{R}, q^{\epsilon}\right) \rtimes \Gamma\right)$ are isomorphic, by isomorphisms depending continuously on $\epsilon \in[-1,1]$. We have two families of representations on $V, \pi \circ \zeta_{\epsilon}$ and $\tilde{\sigma}_{\epsilon}(\pi)$, which agree at $\epsilon=1$ and all whose matrix coefficients are continuous in $\epsilon$. Since a finite dimensional semisimple algebra has only finitely many equivalence classes of representations on $V$, such equivalence classes are rigid under continuous deformations. Therefore $\pi \circ \zeta_{\epsilon} \cong \tilde{\sigma}_{\epsilon}(\pi)$ for all $\epsilon \in[-1,1]$. Now Lemma 4.2 .3 (or a simpler version of the above argument) shows that $\pi^{\Gamma}(P, \delta, t) \circ \zeta_{\epsilon} \cong \pi_{\epsilon}^{\Gamma}\left(P, \tilde{\sigma}_{\epsilon}(\delta), t\right)$, concluding the proof of (e).

Property (a) is a consequence of (e), Corollary 4.2 .2 and Lemma 3.1.1.b. According to Opd2, Lemma 5.5] the scaling maps $\tilde{\sigma}_{\epsilon}$ preserve the Plancherel measure $\mu_{P l}$ for $\epsilon>0$. By part (e) and Theorem 4.2.1, so do the maps $\pi \mapsto \pi \circ \zeta_{\epsilon}$. Now the last part of Theorem 3.2 .2 shows that

$$
\tau\left(\zeta_{\epsilon}(h)\right)=\tau(h) \text { for all } \epsilon \in(0,1], h \in \mathcal{H}_{\epsilon} \rtimes \Gamma .
$$

By part (d) there is a small $\epsilon^{\prime}>0$ such that (4.26) also holds for all $\epsilon \in\left[-\epsilon^{\prime}, 0\right]$. Moreover, from the end of the proof of Lemma 4.3.2 we see that we can make the maps from (d) analytic at any given $\epsilon \in[-1,1]$. Of course this involves some choices, but they do not influence $\tau$ because they all come from conjugation in certain algebras. Therefore (4.26) extends to all $\epsilon \in[-1,1]$.

As concerns the injectivity of $\zeta_{\epsilon}$, suppose that $h \in \operatorname{ker}\left(\zeta_{\epsilon}\right)$. Then $M(t, h)=0$ for all unitary principal series representations $M(t)$. Since $T_{u n}$ is Zariski-dense in $T$, Lemma 3.1.2 for $\mathcal{H}_{\epsilon} \rtimes \Gamma$ shows that $h=0$.

Notice that for $\epsilon<0$ composition with $\zeta_{\epsilon}$ does not preserve the local (in the dual space) traces $\mu_{P l}(\xi) \operatorname{tr} \pi^{\Gamma}(\xi)$, because by Theorem 3.2.2 and Proposition 4.4 .1 $\tilde{\sigma}_{\epsilon}$ does not preserve the support of the Plancherel measure.

In general $\zeta_{\epsilon}\left(\mathcal{H}_{\epsilon} \rtimes \Gamma\right)$ is not contained in $\mathcal{H} \rtimes \Gamma$, for two reasons: $\zeta_{\delta, \epsilon}$ usually does not preserve polynomiality, and not every polynomial section is in the image of $\mathcal{F}$. For $\epsilon \geq 0$ the $\zeta_{\epsilon}$ extend continuously to $\mathcal{S}\left(\mathcal{R}, q^{\epsilon}\right) \rtimes \Gamma$ :

Theorem 4.4.2. For $\epsilon \in[0,1]$ there exist homomorphisms of Fréchet *-algebras

$$
\begin{array}{rr}
\zeta_{\epsilon}: & \mathcal{S}\left(\mathcal{R}, q^{\epsilon}\right) \rtimes \Gamma \quad \rightarrow \quad \mathcal{S}(\mathcal{R}, q) \rtimes \Gamma, \\
\zeta_{\epsilon}: & C^{*}\left(\mathcal{R}, q^{\epsilon}\right) \rtimes \Gamma \quad \rightarrow \quad C^{*}(\mathcal{R}, q) \rtimes \Gamma,
\end{array}
$$

with the following properties:

(a) $\zeta_{\epsilon}$ is an isomorphism for $\epsilon>0$, and $\zeta_{0}$ is injective.

(b) $\zeta_{1}$ is the identity.

(c) $\zeta_{\epsilon}(h)=h$ for all $h \in \mathcal{S}(Z(W))$. 
(d) Let $x \in C^{*}(W \rtimes \Gamma)$ and let $h=\sum_{w \in W \rtimes \Gamma} h_{w} N_{w}$ with $p_{n}(h)<\infty$ for all $n \in \mathbb{N}$. Then the following maps are continuous:

$$
\begin{aligned}
& {[0,1] \rightarrow \mathcal{S}(\mathcal{R}, q) \rtimes \Gamma, \quad \epsilon \quad \zeta_{\epsilon}(h),} \\
& {[0,1] \rightarrow B\left(L^{2}(\mathcal{R}, q)\right), \quad \epsilon \quad \zeta_{\epsilon}^{-1} \zeta_{0}(x) .}
\end{aligned}
$$

(e) For all $\pi \in \operatorname{Mod}_{f}(\mathcal{S}(\mathcal{R}, q) \rtimes \Gamma)$ the representations $\pi \circ \zeta_{\epsilon}$ and $\tilde{\sigma}_{\epsilon}(\pi)$ are equivalent.

(f) $\zeta_{\epsilon}$ preserves the trace $\tau$.

Proof. For any $(P, \delta) \in \Delta$ the representation $\tilde{\sigma}_{\epsilon}(\delta)$, although not necessarily irreducible if $\epsilon=0$, is certainly completely reducible, being unitary. Hence by Theorem 3.2 .2 every irreducible constituent $\pi_{1}$ of $\tilde{\sigma}_{\epsilon}(\delta)$ is a direct summand of

$$
\operatorname{Ind}_{\mathcal{H}_{\epsilon, P}^{P_{1}}}^{\mathcal{H}_{\epsilon, P}}\left(\delta_{1} \circ \phi_{t_{1}, \epsilon}\right)
$$

for a $P_{1} \subset P$, a discrete series representation $\delta_{1}$ of $\mathcal{H}\left(\mathcal{R}_{P_{1}}, q^{\epsilon}\right)$ and a

$$
t_{1} \in \operatorname{Hom}_{\mathbb{Z}}\left(\left(X_{P}\right)^{P_{1}}, S^{1}\right)=\operatorname{Hom}_{\mathbb{Z}}\left(X /\left(X \cap\left(P^{\vee}\right)^{\perp}+\mathbb{Q} P_{1}\right), S^{1}\right) \subset T_{u n} .
$$

Consequently, $\pi_{\epsilon}\left(P, \pi_{1}, t\right)$ is a direct summand of

$$
\operatorname{Ind}_{\mathcal{H}_{\epsilon}^{P}}^{\mathcal{H}_{\epsilon}}\left(\operatorname{Ind}_{\mathcal{H}_{\epsilon, P}^{P_{1}}}^{\mathcal{H}_{\epsilon, P}}\left(\delta_{1} \circ \phi_{t_{1}, \epsilon}\right) \circ \phi_{t, \epsilon}\right)=\pi_{\epsilon}\left(P_{1}, \delta_{1}, t t_{1}\right) .
$$

In particular, for $t \in T_{u n}^{P}$ every matrix coefficient of $\pi_{\epsilon}^{\Gamma}\left(P, \tilde{\sigma}_{\epsilon}(\delta), t\right)$ appears in the Fourier transform of $\mathcal{S}\left(\mathcal{R}, q^{\epsilon}\right) \rtimes \Gamma$, so (4.14) extends to the respective Schwartz and $C^{*}$-completions, as required. By Corollary 4.2.2. b and (4.25) these maps are isomorphisms if $\epsilon>0$. Since (4.24) extends continuously to the appropriate $C^{*}$ completions, so does the algebra homomorphism $\zeta_{\epsilon}$ from Proposition 4.4.1.

Properties (b), (c), (e) and (f) are direct consequences of the corresponding parts of Proposition 4.4.1.

To see that $\zeta_{0}$ remains injective we vary on the proof of Proposition 4.4.1. By (e) the family of representations

$$
I_{t} \circ \zeta_{\epsilon} \cong \pi_{\epsilon}^{\Gamma}\left(\emptyset, \tilde{\sigma}_{\epsilon}\left(\delta_{\emptyset}\right), t\right)=\pi_{\epsilon}^{\Gamma}\left(\emptyset, \delta_{\emptyset}, t\right)
$$

with $t \in T_{u n}$ becomes precisely the unitary principal series of $W \rtimes \Gamma$ when $\epsilon \rightarrow 0$. By Lemma 2.2.2 and Frobenius reciprocity every irreducible tempered representation of $\mathcal{H}\left(\mathcal{R}, q^{0}\right) \rtimes \Gamma=\mathbb{C}[W \rtimes \Gamma]$ is a quotient of a unitary principal series representation. Hence every element of $C^{*}\left(\mathcal{R}, q^{0}\right) \rtimes \Gamma=C^{*}(W \rtimes \Gamma)$ that lies in the kernel of $\zeta_{0}$ annihilates all irreducible tempered $W \rtimes \Gamma$-representations, and must be 0 .

The assumptions in (d) mean that we can consider $h$ as an element of $\mathcal{S}\left(\mathcal{R}, q^{\epsilon}\right) \rtimes$ $\Gamma$ for every $\epsilon$. Moreover, the sum $\sum_{w \in W \rtimes \Gamma} h_{w} N_{w}$ converges uniformly to $h$ in $\mathcal{S}\left(\mathcal{R}, q^{\epsilon}\right) \rtimes \Gamma$. For every finite partial sum $h^{\prime}$ the map $\epsilon \mapsto \phi_{\epsilon}\left(h^{\prime}\right)$ is continuous by Proposition 4.4.1.e, so this also holds for $h$ itself.

For $\epsilon \in(0,1]$ we consider

$$
\zeta_{\epsilon}^{-1} \zeta_{0}(x)-x=\mathcal{F}_{\epsilon}^{-1}\left(\bigoplus_{(P, \delta) \in \Delta} \zeta_{P, \delta, \epsilon}^{-1} \zeta_{P, \delta, 0}\left(\mathcal{F}_{0}(x)\right)-\mathcal{F}_{\epsilon}(x)\right) .
$$

Since $\zeta_{P, \delta, 0}$ is invertible, both $\zeta_{P, \delta, \epsilon}^{-1} \zeta_{P, \delta, 0}\left(\mathcal{F}_{0}(x)\right)$ and $\mathcal{F}_{\epsilon}(x)$ are continuous in $\epsilon$ and converge to $\mathcal{F}_{0}(x)$ as $\epsilon \downarrow 0$. The continuity of $\mathcal{F}_{\epsilon}$ with respect to $\epsilon$ implies that the $\mathcal{F}_{\epsilon}^{-1}$ are also continuous with respect to $\epsilon$, so $\epsilon \mapsto \zeta_{\epsilon}^{-1} \zeta_{0}(x)$ is continuous. 
The expression between the large brackets in (4.27) also depends continuously on $\epsilon$ and converges to 0 as $\epsilon \downarrow 0$. Furthermore,

$$
\mathcal{F}_{\epsilon}^{-1}: \bigoplus_{(P, \delta) \in \Delta}\left(C\left(T_{u n}^{P}\right) \otimes \operatorname{End}_{\mathbb{C}}\left(\mathbb{C}\left[\Gamma W^{P}\right] \otimes V_{\delta}\right)\right)^{\mathcal{G}_{P, \tilde{\epsilon}_{\epsilon}(\delta)}} \rightarrow B\left(L^{2}(\mathcal{R}, q)\right)
$$

is a homomorphism of $C^{*}$-algebras, so it cannot increase the norms. Therefore

$$
\lim _{\epsilon \downarrow 0}\left(\zeta_{\epsilon}^{-1} \zeta_{0}(x)-x\right)=0 \text { in } B\left(L^{2}(\mathcal{R}, q)\right) .
$$

The homomorphisms from Theorem 4.4.2 are by no means natural, the construction involves a lot of arbitrary choices. Nevertheless, one can prove Sol3, Lemma 5.22 ] that it is possible to interpolate continuously between two such homomorphisms, obtained from different choices. In other words, the homotopy class of $\zeta_{\epsilon}: \mathcal{S}\left(\mathcal{R}, q^{\epsilon}\right) \rtimes \Gamma \rightarrow \mathcal{S}(\mathcal{R}, q) \rtimes \Gamma$ is canonical.

It is quite remarkable that $\zeta_{0}$ preserves the trace $\tau$, because the measure space $\left(\Xi_{u n}, \mu_{P l}\right)$ differs substantially from $T_{u n}$ with the Haar measure (which corresponds to the algebra $\left.\mathcal{S}(X) \rtimes W_{0}^{\prime}\right)$. For example, the former is disconnected and can have isolated points, while the latter is a connected manifold. So the preservation of the trace already indicates that it is not possible to separate all components of $\Xi_{\text {un }} / \mathcal{G}$ using only elements from the image of $\zeta_{0}$.

Corollary 4.4.3. For $\pi \in \operatorname{Irr}(\mathcal{S}(\mathcal{R}, q) \rtimes \Gamma)$ we have $\zeta^{*}(\pi) \cong \tilde{\sigma}_{0}(\pi) \cong \pi \circ \zeta_{0}$, where $\zeta^{*}$ is as in Section 2.3. The map $\zeta_{0}$ induces a linear bijection

$$
G_{\mathbb{Q}}\left(\zeta_{0}\right): G_{\mathbb{Q}}(\mathcal{S}(\mathcal{R}, q) \rtimes \Gamma) \rightarrow G_{\mathbb{Q}}(\mathcal{S}(W) \rtimes \Gamma) .
$$

Proof. The first claim follows from Theorem 4.4.2 e and Lemma4.2.3 b. The second statement follows from the first and Theorem 2.3.1.a

\section{Noncommutative Geometry}

Affine Hecke algebras have some clear connections with noncommutative geometry. Already classical is the isomorphism between an affine Hecke algebra (with one formal parameter $\mathbf{q}$ ) and the equivariant $K$-theory of a Steinberg variety; see Lus2, KaLu2, ChGi]. Of a more analytic nature is $K$-theory of the $C^{*}$-completion $C^{*}(\mathcal{R}, q)$ of $\mathcal{H}(\mathcal{R}, q)$. It is relevant for the representation theory of affine Hecke algebras because topological $K$-theory is built from finitely generated projective modules. Since $K$-theory tends to be invariant under small perturbations, it is expected [Ply1, $\mathrm{BCH}]$ that $K_{*}\left(C^{*}(\mathcal{R}, q)\right)$ does not depend on $q$. We prove this modulo torsion (Theorem 5.1.4).

For the algebra $\mathcal{H}(\mathcal{R}, q)$ periodic cyclic homology is more suitable than $K$-theory. Although periodic cyclic homology is not obviously related to representation theory, there is a link for certain classes of algebras [Sol6]. From [BaNi] it is known that $H P_{*}(\mathcal{H}(\mathcal{R}, q)) \cong H P_{*}(\mathbb{C}[W])$ when $q$ is an equal parameter function, but the proof is by no means straightforward.

We connect these two theories via the Schwartz completion of $\mathcal{H}(\mathcal{R}, q)$. For this algebra both topological $K$-theory and periodic cyclic homology are meaningful invariants. Notwithstanding the different nature of the algebras $\mathcal{H}(\mathcal{R}, q)$ and $\mathcal{S}(\mathcal{R}, q)$, they have the same periodic cyclic homology (Theorem 5.2.1). We deduce the existence of natural isomorphisms

$$
H P_{*}(\mathcal{H}(\mathcal{R}, q)) \cong H P_{*}(\mathcal{S}(\mathcal{R}, q)) \cong K_{*}(\mathcal{S}(\mathcal{R}, q)) \otimes_{\mathbb{Z}} \mathbb{C} \cong K_{*}\left(C^{*}(\mathcal{R}, q)\right) \otimes_{\mathbb{Z}} \mathbb{C} .
$$


Moreover, the scaling maps from Section 4 provide isomorphisms from these vector spaces to the corresponding invariants of group algebras of $W$ (Corollary [5.2.2). Notice the similarity with the ideas of [BHP, Sol4].

Our method of proof actually shows that $\mathcal{S}(\mathcal{R}, q)$ and $\mathcal{S}(W)$ are geometrically equivalent (Lemma 5.3.1), a term coined by Aubert, Baum and Plymen ABP1 to formulate a conjecture for Hecke algebras of $p$-adic groups. This conjecture (which we call the ABP-conjecture) describes the structure of Bernstein components in the smooth dual of a reductive $p$-adic group. Translated to affine Hecke algebras this conjecture says among others that the dual of $\mathcal{H}$ can be parametrized with the extended quotient $\widetilde{T} / W_{0}$. The topological space $\operatorname{Irr}(\mathcal{H}(\mathcal{R}, q))$, with its central character map to $T / W_{0}$, should then be obtained from $\widetilde{T} / W_{0}$, with its canonical projection onto $T / W_{0}$, via translating the components of $\widetilde{T} / W_{0}$ in algebraic dependence on $q$. We verify the ABP-conjecture for all affine Hecke algebras with positive parameters, possibly extended with a group of diagram automorphisms (Theorem 5.4.2). Hence the ABP-conjecture holds for all Bernstein components of $p$-adic groups whose Hecke algebras are known to be Morita equivalent to affine Hecke algebras.

In the final section we calculate in detail what happens for root data with $R_{0}$ of type $B_{2} / C_{2}$. Interestingly, this also shows that the representation theory of $\mathcal{H}(\mathcal{R}, q)$ seems to behave very well under general deformations of the parameter function $q$.

5.1. Topological $K$-theory. By means of the canonical basis $\left\{N_{w}: w \in W \rtimes \Gamma\right\}$ we can identify the topological vector spaces underlying the algebras $\mathcal{S}(\mathcal{R}, q) \rtimes \Gamma$ for all positive parameter functions $q$. It is clear from the rules (1.4) that the multiplication in affine Hecke algebras depends continuously on $q$, in some sense. To make that precise, one endows finite dimensional subspaces of $\mathcal{H}(\mathcal{R}, q) \rtimes \Gamma$ with their standard topology, and one defines a topology on the space of positive parameter functions by identifying them with tuples of positive real numbers. This can be extended to the Schwartz completions: by OpSo2, Lemma A.8] the multiplication in $\mathcal{S}(\mathcal{R}, q) \rtimes \Gamma$ is continuous in $q$, with respect to the Fréchet topology on $\mathcal{S}(\mathcal{R}, q) \rtimes \Gamma$. This opens the possibility to investigate this field of Fréchet algebras with analytic techniques that relate the algebras for different $q$ 's, a strategy that was used at some crucial points in OpSo2.

We denote the topological $K$-theory of a Fréchet algebra $A$ by $K_{*}(A)=K_{0}(A) \oplus$ $K_{1}(A)$. Since $\mathcal{S}(\mathcal{R}, q) \rtimes \Gamma$ is closed under the holomorphic functional calculus of $C^{*}(\mathcal{R}, q) \rtimes \Gamma$ OpSo2, Theorem A.7], the density theorem [Bos, Théorème A.2.1] tells us that the inclusion $\mathcal{S}(\mathcal{R}, q) \rtimes \Gamma \rightarrow C^{*}(\mathcal{R}, q) \rtimes \Gamma$ induces an isomorphism

$$
K_{*}(\mathcal{S}(\mathcal{R}, q) \rtimes \Gamma) \cong K_{*}\left(C^{*}(\mathcal{R}, q) \rtimes \Gamma\right) .
$$

$K$-theory for $C^{*}$-algebras is homotopy-invariant, so it is natural to expect the following:

Conjecture 5.1.1. For any positive parameter function $q$ the abelian groups $K_{*}\left(C^{*}(W) \rtimes \Gamma\right)$ and $K_{*}\left(C^{*}(\mathcal{R}, q) \rtimes \Gamma\right)$ are naturally isomorphic.

This conjecture stems from Higson and Plymen (see [Ply1, 6.4] and [BCH, 6.21]), at least when $\Gamma=\{\operatorname{id}\}$ and $q$ is constant on $S^{\text {aff }}$. It is similar to the ConnesKasparov conjecture for Lie groups; see [BCH, Sections 4-6] for more background. Independently, Opdam Opd2, Section 1.0.1] formulated Conjecture 5.1.1] for unequal parameters. We will discuss its relevance for the representation theory of 
affine Hecke algebras, and we will prove a slightly weaker version, obtained by applying the functor $\otimes_{\mathbb{Z}} \mathbb{Q}$.

Recall [Phi] that for any unital Fréchet algebra $A, K_{0}(A)$ (respectively $K_{1}(A)$ ) is generated by idempotents (respectively invertible elements) in matrix algebras $M_{n}(A)$. The $K$-groups are obtained by taking equivalence classes with respect to the relation generated by stabilization and homotopy equivalence.

Lemma 5.1.2. Suppose that

$$
\kappa_{\epsilon}: K_{*}\left(C^{*}(W) \rtimes \Gamma\right) \rightarrow K_{*}\left(C^{*}(\mathcal{R}, q) \rtimes \Gamma\right) \quad \epsilon \in[0,1]
$$

is a family of group homomorphisms with the following property.

For every idempotent $e_{0} \in M_{n}\left(\mathcal{S}\left(\mathcal{R}, q^{0}\right) \rtimes \Gamma\right)=M_{n}(\mathcal{S}(W) \rtimes \Gamma)$ (resp. invertible element $x_{0} \in M_{n}\left(\mathcal{S}\left(\mathcal{R}, q^{0}\right) \rtimes \Gamma\right)$ ) there exists a $C^{*}$-norm-continuous path $\epsilon \mapsto e_{\epsilon}$ (resp. $\left.\epsilon \mapsto x_{\epsilon}\right)$ in the Fréchet space underlying $M_{n}(\mathcal{S}(\mathcal{R}, q) \rtimes \Gamma)$, such that $\kappa_{\epsilon}\left[e_{0}\right]=$ $\left[e_{\epsilon}\right]\left(\right.$ resp. $\left.\kappa_{\epsilon}\left[x_{0}\right]=\left[x_{\epsilon}\right]\right)$.

Then $\kappa_{\epsilon}=K_{*}\left(\zeta_{\epsilon}^{-1} \zeta_{0}\right)$, with $\zeta_{\epsilon}: C^{*}\left(\mathcal{R}, q^{\epsilon}\right) \rtimes \Gamma \rightarrow C^{*}(\mathcal{R}, q) \rtimes \Gamma$ as in Theorem 4.4 .2 .

By " $C^{*}$-norm-continuous" we mean that the path in $B\left(L^{2}(\mathcal{R}, q)\right)$ defined by mapping $\epsilon$ to the operator of left multiplication by $e_{\epsilon}$ (with respect to $q^{\epsilon}$ ), is continuous. It follows from OpSo2, Proposition A.5] that every Fréchet-continuous path is $C^{*}$ norm-continuous. By Theorem 4.4.2 d the maps $K_{*}\left(\zeta_{\epsilon}^{-1} \zeta_{0}\right)$ have the property that the $\kappa_{\epsilon}$ are supposed to possess, so at least the statement is meaningful.

Proof. By definition $K_{*}\left(\zeta_{\epsilon}^{-1} \zeta_{0}\right)\left[e_{0}\right]=\left[\zeta_{\epsilon}^{-1} \zeta_{0}\left(e_{0}\right)\right]$, where we extend the $\zeta_{\epsilon}$ to matrix algebras over $C^{*}\left(\mathcal{R}, q^{\epsilon}\right)$ in the obvious way. The paths $\epsilon \rightarrow e_{\epsilon}$ and $\epsilon \rightarrow \zeta_{\epsilon}^{-1} \zeta_{0}\left(e_{0}\right)$ are both $C^{*}$-norm-continuous, so we can find $\epsilon^{\prime}>0$ such that

$$
\left\|\zeta_{\epsilon}^{-1} \zeta_{0}\left(e_{0}\right)-e_{\epsilon}\right\|<\left\|2 e_{0}-1\right\|^{-1}=\left\|2 \zeta_{\epsilon}^{-1} \zeta_{0}\left(e_{0}\right)-1\right\|^{-1} \text { for all } \epsilon \leq \epsilon^{\prime} .
$$

Then by [Bla, Proposition 4.3.2] $e_{\epsilon}$ and $\zeta_{\epsilon}^{-1} \zeta_{0}\left(e_{0}\right)$ are connected by a path of idempotents in $M_{n}\left(C^{*}\left(\mathcal{R}, q^{\epsilon}\right) \rtimes \Gamma\right)$, so

$$
\kappa_{\epsilon}\left[e_{0}\right]=\left[e_{\epsilon}\right]=\left[\zeta_{\epsilon}^{-1} \zeta_{0}\left(e_{0}\right)\right]=K_{*}\left(\zeta_{\epsilon}^{-1} \zeta_{0}\right)\left[e_{0}\right] \text { for all } \epsilon \leq \epsilon^{\prime} .
$$

For $\epsilon \geq \epsilon^{\prime}$,

$$
K_{*}\left(\zeta_{\epsilon}^{-1} \zeta_{0}\right)\left[e_{0}\right]=K_{*}\left(\zeta_{\epsilon}^{-1} \zeta_{\epsilon^{\prime}}\right) K_{*}\left(\zeta_{\epsilon^{\prime}}^{-1} \zeta_{0}\right)\left[e_{0}\right]=K_{*}\left(\zeta_{\epsilon}^{-1} \zeta_{\epsilon^{\prime}}\right)\left[e_{\epsilon^{\prime}}\right] .
$$

By parts (a) and (d) of Theorem 4.4.2 $K_{*}\left(\zeta_{\epsilon}^{-1} \zeta_{\epsilon^{\prime}}\right)\left(\epsilon \geq \epsilon^{\prime}\right)$ is the only family of maps $\left.K_{0}\left(C^{*}\left(\mathcal{R}, q^{\epsilon^{\prime}}\right)\right) \rtimes \Gamma\right) \rightarrow K_{0}\left(C^{*}(\mathcal{R}, q) \rtimes \Gamma\right)$ that comes from continuous paths of idempotents.

Now $K_{1}$. Choose $\epsilon^{\prime}>0$ such that

$$
\left\|\zeta_{\epsilon}^{-1} \zeta_{0}\left(x_{0}\right) x_{\epsilon}^{-1}-1\right\|<1 \quad \text { for all } \epsilon \leq \epsilon^{\prime} .
$$

Then $\zeta_{\epsilon}^{-1} \zeta_{0}\left(x_{0}\right) x_{\epsilon}^{-1}$ is homotopic to 1 in $G L_{n}\left(C^{*}\left(\mathcal{R}, q^{\epsilon}\right) \rtimes \Gamma\right)$, so

$$
K_{1}\left(\zeta_{\epsilon}^{-1} \zeta_{0}\right)\left[x_{0}\right]=\left[\zeta_{\epsilon}^{-1} \zeta_{0}\left(x_{0}\right)\right]=\left[x_{\epsilon}\right]=\kappa_{\epsilon}\left[x_{0}\right] \text { for all } \epsilon \leq \epsilon^{\prime} .
$$

The argument for $\epsilon \geq \epsilon^{\prime}$ is just as for $K_{0}$.

This lemma says that the map

$$
K_{*}\left(\zeta_{0}\right): K_{*}\left(C^{*}(W) \rtimes \Gamma\right) \rightarrow K_{*}\left(C^{*}(\mathcal{R}, q) \rtimes \Gamma\right)
$$

is natural: it does not really depend on $\zeta_{0}$, only on the topological properties of idempotents and invertible elements in matrix algebras over $\mathcal{S}\left(\mathcal{R}, q^{\epsilon}\right) \rtimes \Gamma$ with 
$\epsilon \in[0,1]$. To prove that $K_{*}\left(\zeta_{0}\right)$ becomes an isomorphism after tensoring with $\mathbb{Q}$, we need some preparations of a more general nature.

For topological spaces $Y \subset X$ and a topological algebra $A$ we write

$$
C_{0}(X, Y ; A)=\left\{f: X \rightarrow A \mid f \text { is continuous and }\left.f\right|_{Y}=0\right\} \text {. }
$$

We omit $Y$ (resp. $A$ ) from the notation if $Y=\emptyset($ resp. $A=\mathbb{C})$. A $C(X)$-algebra $B$ is a $\mathbb{C}$-algebra endowed with a unital algebra homomorphism from $C(X)$ to the center of the multiplier algebra of $B$. A morphism of $C(X)$-algebras is an algebra homomorphism that is also a $C(X)$-module map.

Lemma 5.1.3. Let $\Sigma$ be a finite simplicial complex, let $A$ and $B$ be $C(\Sigma)$-Banachalgebras and let $\phi: A \rightarrow B$ a morphism of $C(\Sigma)$-Banach-algebras. Suppose that

(a) for every simplex $\sigma$ of $\Sigma$ there are finite dimensional $\mathbb{C}$-algebras $A_{\sigma}$ and $B_{\sigma}$ such that

$$
C_{0}(\sigma, \delta \sigma) A \cong C_{0}\left(\sigma, \delta \sigma ; A_{\sigma}\right) \text { and } C_{0}(\sigma, \delta \sigma) B \cong C_{0}\left(\sigma, \delta \sigma ; B_{\sigma}\right) ;
$$

(b) for every $x \in \sigma \backslash \delta \sigma$ the localized map $\phi(x): A_{\sigma} \rightarrow B_{\sigma}$ induces an isomorphism on $K$-theory.

Then $K_{*}(\phi): K_{*}(A) \stackrel{\sim}{\longrightarrow} K_{*}(B)$ is an isomorphism.

Proof. Let $\Sigma^{n}$ be the $n$-skeleton of $\Sigma$ and consider the ideals

$$
I_{0}=C(\Sigma) \supset I_{1}=C\left(\Sigma ; \Sigma^{0}\right) \supset \cdots \supset I_{n}=C_{0}\left(\Sigma, \Sigma^{n-1}\right) \supset \cdots .
$$

They give rise to ideals $I_{n} A$ and $I_{n} B$. Because $\Sigma$ is finite, all these ideals are 0 for large $n$. We can identify

$$
\begin{aligned}
& I_{n} A / I_{n+1} A \cong C_{0}\left(\Sigma^{n}, \Sigma^{n-1}\right) A \\
& \cong \bigoplus_{\sigma \in \Sigma: \operatorname{dim} \sigma=n} A C_{0}(\sigma, \delta \sigma):=\bigoplus_{\sigma \in \Sigma: \operatorname{dim} \sigma=n} C_{0}\left(\sigma, \delta \sigma ; A_{\sigma}\right),
\end{aligned}
$$

and similarly for $B$. Because $\phi$ is $C(\Sigma)$-linear, it induces homomorphisms

$$
\phi(\sigma): C_{0}\left(\sigma, \delta \sigma ; A_{\sigma}\right) \rightarrow C_{0}\left(\sigma, \delta \sigma ; B_{\sigma}\right) .
$$

By the additivity of and the excision property of the $K$-functor (see e.g. Bla, Theorem 9.3.1]), it suffices to show that every $\phi(\sigma)$ induces an isomorphism on $K$-theory. Let $x$ be any interior point of $\sigma$. Because $\sigma \backslash \delta \sigma$ is contractible, $\phi_{\sigma}$ is homotopic to $\operatorname{id}_{C_{0}(\sigma, \delta \sigma)} \otimes \phi\left(x_{\sigma}\right)$. By assumption the latter map induces an isomorphism on $K$-theory. With the homotopy invariance of $K$-theory it follows that

$$
K_{*}(\phi(\sigma))=K_{*}\left(\operatorname{id}_{C_{0}(\sigma, \delta \sigma)} \otimes \phi\left(x_{\sigma}\right)\right)
$$

is an isomorphism.

Obviously, this lemma is in no way optimal: one can generalize it to larger classes of algebras and one can relax the finiteness assumption on $\Sigma$. Because we do not need it, we will not bother to write down such generalizations. What we will need, however, is that Lemma 5.1 .3 is also valid for similar functors; in particular, for $A \mapsto K_{*}(A) \otimes_{\mathbb{Z}} \mathbb{C}$.

Theorem 5.1.4. The map

$$
K_{*}\left(\zeta_{0}\right) \otimes \operatorname{id}_{\mathbb{Q}}: K_{*}\left(C^{*}(W) \rtimes \Gamma\right) \otimes_{\mathbb{Z}} \mathbb{Q} \rightarrow K_{*}\left(C^{*}(\mathcal{R}, q) \rtimes \Gamma\right) \otimes_{\mathbb{Z}} \mathbb{Q}
$$

is a $\mathbb{Q}$-linear bijection. 
Proof. Consider the projection

$$
\operatorname{pr}: \Xi_{u n} / \mathcal{G} \rightarrow T_{u n} / W_{0}^{\prime}, \mathcal{G}(P, \delta, t) \mapsto W_{0}^{\prime} r|r|^{-1} t,
$$

where $W\left(R_{P}\right) r \in T_{P} / W\left(R_{P}\right)$ is the central character of $\delta$. With this projection and Theorem 3.2.2 we make $C^{*}(\mathcal{R}, q)$ into a $C\left(T_{u n} / W_{0}^{\prime}\right)$-algebra. By Theorem 4.4.2.e $\zeta_{0}: C^{*}(W) \rtimes \Gamma \rightarrow C^{*}(\mathcal{R}, q) \rtimes \Gamma$ is a morphism of $C\left(T_{u n} / W_{0}^{\prime}\right)-C^{*}$-algebras. Choose a triangulation $\Sigma$ of $T_{u n}$, such that:

- $w(\sigma) \in \Sigma$ for every simplex $\sigma \in \Sigma$ and every $w \in W_{0}^{\prime}$;

- $T_{u n}^{G}$ is a subcomplex of $\Sigma$, for every subgroup $G \subset W_{0}^{\prime}$;

- the star of any simplex $\sigma$ is $W_{0, \sigma}^{\prime}$-equivariantly contractible.

Then $\Sigma / W_{0}^{\prime}$ is a triangulation of $T_{u n} / W_{0}^{\prime}$. From Theorem 3.2 .2 and the proof of Sol1, Lemma 7] we see that $A=C^{*}(W) \rtimes \Gamma$ and $B=C^{*}(\mathcal{R}, q) \rtimes \Gamma$ and are of the form required in condition (a) of Lemma 5.1.3. For any $u \in T_{u n}$ we write

$$
\begin{aligned}
& A_{u}:=C^{*}(W) \rtimes \Gamma / \operatorname{ker} I_{u}, \\
& B_{u}:=\bigoplus_{\mathcal{G} \xi \in \Xi_{u n} / \mathcal{G}, \operatorname{pr}(\xi)=W_{0}^{\prime} u} C^{*}(\mathcal{R}, q) \rtimes \Gamma / \operatorname{ker} \pi^{\Gamma}(\xi) .
\end{aligned}
$$

Condition (b) of Lemma 5.1 .3 for $K_{*}(?) \otimes_{\mathbb{Z}} \mathbb{Q}$ means that the map $\zeta_{0}\left(W_{0}^{\prime} u\right): A_{u} \rightarrow$ $B_{u}$ should induce an isomorphism

$$
K_{*}\left(\zeta_{0}\left(W_{0}^{\prime} u\right)\right): K_{*}\left(A_{u}\right) \otimes_{Z} \mathbb{Q} \rightarrow K_{*}\left(B_{u}\right) \otimes_{\mathbb{Z}} \mathbb{Q} .
$$

As for all finite dimensional semisimple algebras,

$$
K_{*}\left(A_{u}\right)=K_{0}\left(A_{u}\right)=G_{\mathbb{Z}}(A) \quad \text { and } \quad K_{*}\left(B_{u}\right)=K_{0}\left(B_{u}\right)=G_{\mathbb{Z}}\left(B_{u}\right) .
$$

With these identifications $K_{0}\left(\zeta_{0}\left(W_{0}^{\prime} u\right)\right)$ sends a projective module $e M_{n}\left(A_{u}\right)$ to the projective module $\zeta_{0}\left(W_{0}^{\prime} u\right)(e) M_{n}\left(B_{u}\right)$. The free abelian groups $G_{\mathbb{Z}}\left(A_{u}\right)$ and $G_{\mathbb{Z}}\left(B_{u}\right)$ have natural bases consisting of irreducible modules. With respect to these bases the matrix of $K_{0}\left(\zeta_{0}\left(W_{0}^{\prime} u\right)\right)$ is the transpose of the matrix of

$$
\tilde{\sigma}_{0}: G_{\mathbb{Z}}\left(B_{u}\right) \rightarrow G_{\mathbb{Z}}\left(A_{u}\right), \pi \mapsto \pi \circ \zeta_{0}\left(W_{0}^{\prime} u\right) .
$$

By Theorem 2.3.1 a $\tilde{\sigma}_{0} \otimes \operatorname{id}_{\mathbb{Q}}: G_{\mathbb{Q}}\left(A_{u}\right) \rightarrow G_{\mathbb{Q}}\left(B_{u}\right)$ is a bijection, so (5.4) is also a bijection. Now we can apply Lemma 5.1.3, which finishes the proof.

So we proved Conjecture 5.1.1 modulo torsion, which raises the question what information is still contained in the torsion part. It is known that $K^{*}\left(C^{*}(\mathcal{R}, q) \rtimes \Gamma\right)$ is a finitely generated group. Indeed, by [Sol1, Theorem 6] this is the case for all Fréchet algebras of the type described in Theorem 3.2.2. Hence the torsion subgroup of $K^{*}\left(C^{*}(\mathcal{R}, q) \rtimes \Gamma\right)$ is finite. In fact, the author does not know any examples of nontrivial torsion elements in such $K$-groups, but it is conceivable that they exist. It turns out that this is related to the multiplicities of $W_{0}^{\prime}$-representations in Section 2.3. in particular (2.24).

Lemma 5.1.5. The following are equivalent:

(a) $K_{*}\left(\zeta_{0}\right): K_{*}\left(C^{*}(W) \rtimes \Gamma\right) \rightarrow K_{*}\left(C^{*}(\mathcal{R}, q) \rtimes \Gamma\right)$ is an isomorphism.

(b) $K_{0}\left(\zeta_{0}\right): K_{0}\left(C^{*}(W) \rtimes \Gamma\right) \rightarrow K_{0}\left(C^{*}(\mathcal{R}, q) \rtimes \Gamma\right)$ is surjective.

(c) For every $u \in T_{u n}$ the map $\zeta^{*}$ induces a surjection from the Grothendieck group of $\operatorname{Mod}_{f, W_{0}^{\prime} u T_{r s}}(\mathcal{S}(\mathcal{R}, q) \rtimes \Gamma)$ to that of $\operatorname{Mod}_{f, W_{0}^{\prime} u}(W \rtimes \Gamma)$.

(d) The map $\zeta^{*}$ induces a bijection $\zeta_{\mathbb{Z}}^{*}: G_{\mathbb{Z}}(\mathcal{S}(\mathcal{R}, q) \rtimes \Gamma) \rightarrow G_{\mathbb{Z}}(\mathcal{S}(W) \rtimes \Gamma)$. 
Proof. (a) $\Rightarrow$ (b) Obvious.

(b) $\Rightarrow$ (c) We use the notation from the proof of Theorem 5.1.4 in particular, $K_{0}\left(A_{u}\right)$ and $K_{0}\left(B_{u}\right)$ are the Grothendieck groups referred to in (c). We claim that the canonical map

$$
K_{0}\left(C^{*}(\mathcal{R}, q) \rtimes \Gamma\right) \rightarrow K_{0}\left(B_{u}\right)
$$

is surjective. Recall that $K_{0}\left(B_{u}\right)$ is built from idempotents. Given any idempotent $e_{u} \in M_{n}\left(B_{u}\right)$ we want to find an idempotent $e \in M_{n}\left(C^{*}(\mathcal{R}, q) \rtimes \Gamma\right)$ that maps to it. By Theorem 3.2 .2 this means that on every connected component $\left(P, \delta, T_{u n}^{P}\right) / \mathcal{G}_{P, \delta}$ of $\Xi_{u n} / \mathcal{G}$ we have to find an idempotent $e_{P, \delta}$ in

$$
M_{n}\left(C\left(T_{\text {un }}^{P}\right) \otimes \operatorname{End}_{\mathbb{C}}\left(\mathbb{C}\left[\Gamma W^{P}\right] \otimes V_{\delta}\right)\right)^{\mathcal{G}_{P, \delta}},
$$

which in every point of $\operatorname{pr}^{-1}\left(W_{0}^{\prime} u\right) \cap\left(P, \delta, T_{u n}^{P}\right) / \mathcal{G}_{P, \delta}$ takes the value prescribed by $e_{u}$. Recall that the groupoid $\mathcal{G}$ was built from elements of $W_{0}^{\prime}$ and from the groups $K_{P}=T^{P} \cap T_{P}$. The latter elements permute the components of $\Xi_{u n}$ freely, so $\operatorname{pr}^{-1}\left(W_{0}^{\prime} u\right)$ intersects every component of $\Xi_{u n}$ in at most one $\mathcal{G}$-association class. Therefore we can always find such a $e_{P, \delta}$, proving the claim (5.5).

Together with assumption (b) this implies that

$$
K_{0}\left(C^{*}(W) \rtimes \Gamma\right) \stackrel{K_{0}\left(\zeta_{0}\right)}{\longrightarrow} K_{0}\left(C^{*}(\mathcal{R}, q) \rtimes \Gamma\right) \rightarrow K_{0}\left(B_{u}\right)
$$

is surjective. The underlying $C^{*}$-algebra homomorphism factors via $C^{*}(W) \rtimes \Gamma \rightarrow$ $A_{u}$, so

$$
K_{0}\left(\zeta_{0}\left(W_{0}^{\prime} u\right)\right): K_{0}\left(A_{u}\right) \rightarrow K_{0}\left(B_{u}\right)
$$

is also surjective.

(c) $\Rightarrow$ (d) By Corollary $4.4 .3 \zeta_{\mathbb{Z}}^{*}(\pi)=\pi \circ \zeta_{0}$ for all $\pi \in \operatorname{Mod}_{f}(\mathcal{S}(\mathcal{R}, q) \rtimes \Gamma)$. So in the notation of (5.4) $\zeta_{\mathbb{Z}}^{*}$ is the direct sum, over all $W_{0}^{\prime} u \in T_{u n}$, of the maps

$$
G_{\mathbb{Z}}\left(B_{u}\right) \rightarrow G_{\mathbb{Z}}\left(A_{u}\right): \pi \mapsto \pi \circ \zeta_{0}\left(W_{0}^{\prime} u\right) .
$$

As we noticed in the proof of Theorem 5.1.4, the matrix of this map is the transpose of the matrix of (5.6). We showed in the aforementioned proof that the latter map becomes an isomorphism after applying $\otimes_{\mathbb{Z}} \mathbb{Q}$. As $K_{0}\left(A_{u}\right)$ and $K_{0}\left(B_{u}\right)$ are free abelian groups, this implies that $K_{0}\left(\zeta_{0}\left(W_{0}^{\prime} u\right)\right)$ is injective. So under assumption (c) (5.6) is in fact an isomorphism. Hence, with respect to the natural bases it is given by an integral matrix with determinant \pm 1 . Then the same goes for (5.7), so that map is also bijective. Therefore $\zeta_{\mathbb{Z}}^{*}$ is bijective.

(d) $\Rightarrow$ (a) The above shows that under assumption (d) the maps (5.7) and (5.6) are bijections. Since $K_{1}\left(A_{u}\right)=K_{1}\left(B_{u}\right)=0$, we may apply Lemma 5.1 .2

By Corollary 2.1.5, b and property (d) of Theorem 2.3.1, condition (c) of Lemma 5.1.5 can be reformulated as follows: for all $u \in T_{u n}$ the map $\left.\pi \mapsto \pi\right|_{W_{u}^{\prime}}$ induces a bijection from the Grothendieck group of finite dimensional tempered $\mathbb{H}\left(\tilde{\mathcal{R}}_{u}, k_{u}\right) \rtimes$ $W_{F_{u}, u}^{\prime}$-modules with real central character to $G_{\mathbb{Z}}\left(W_{u}^{\prime}\right)$.

According to [Ciu, Corollary 3.6] this statement is valid for all graded Hecke algebras of "geometric type". Hence Conjecture 5.1.1 holds, including torsion, for many important examples of affine Hecke algebras.

In particular, let $I$ be an Iwahori subgroup of a split reductive $p$-adic group $G$ with root datum $\mathcal{R}$, as in Section [1.6] By Ply2 the completion $C_{r}^{*}(G, I)$ of $\mathcal{H}(G, I)$ is isomorphic to $C^{*}(\mathcal{R}, q)$, where $q$ is some prime power. It is interesting to 
combine Conjecture 5.1.1 with the Baum-Connes conjecture. Let $\beta G$ be the affine Bruhat-Tits building of $G$ and identify $\mathfrak{a}^{*}$ with an apartment. The Baum-Connes conjecture for groups like $G$ and $W$ was proven by V. Lafforgue [Laf]; see also [Sol4]. (For $W$ it can of course be done more elementarily.) We obtain a diagram

$$
\begin{array}{rllc}
K_{*}^{W}\left(\mathfrak{a}^{*}\right) \rightarrow K_{*}\left(C_{r}^{*}(W)\right) & \rightarrow K_{*}\left(C^{*}(\mathcal{R}, q)\right) & \rightarrow & K_{*}\left(C_{r}^{*}(G, I)\right) \\
& & & \\
K_{*}^{G}(\beta G) & \rightarrow K_{*}\left(C_{r}^{*}(G)\right) & \rightarrow & \bigoplus_{\mathfrak{s} \in \mathfrak{B}(G)} K_{*}\left(C_{r}^{*}(G)_{\mathfrak{s}}\right)
\end{array}
$$

in which all the horizontal maps are natural isomorphisms, while the vertical maps pick the factor of $K_{*}\left(C_{r}^{*}(G)\right)$ corresponding to the Iwahori-spherical component in $\mathfrak{B}(G)$. For the group $G=G L_{n}(\mathbb{F})$ this goes back to [Ply1]. Notice that (5.8) realizes $K_{*}^{W}\left(\mathfrak{a}^{*}\right)$ as a direct summand of $K_{*}^{G}(\beta G)$, which is by no means obvious in equivariant $K$-homology.

5.2. Periodic cyclic homology. Periodic cyclic homology is rather similar to topological $K$-theory, but the former functor is defined on larger classes of algebras. For example one can take the periodic cyclic homology of nontopological algebras like $\mathcal{H}(\mathcal{R}, q)$, while it is much more difficult to make sense of the topological $K$-theory of affine Hecke algebras without completing them. By definition the periodic cyclic homology of an algebra over a field $\mathbb{F}$ is an $\mathbb{F}$-vector space. Whereas topological $K$-theory for $C^{*}$-algebras is the generalization of $K$-theory for topological spaces, periodic cyclic homology for noncommutative algebras can be regarded as the analogue of De Rham cohomology for manifolds.

In [Sol6, Theorem 3.3] the author proved with homological-algebraic techniques that the periodic cyclic homology of an (extended) graded Hecke algebra $\mathbb{H}(\tilde{\mathcal{R}}, k) \rtimes \Gamma$ does not depend on the parameter function $k$. Subsequently, he translated this into a representation-theoretic statement, which we already used in (2.24): the collection of irreducible tempered $\mathbb{H}(\tilde{\mathcal{R}}, k) \rtimes \Gamma$-representations with real central character forms a basis of $G_{\mathbb{Q}}\left(W_{0} \rtimes \Gamma\right)$.

We will devise a reversed chain of arguments for affine Hecke algebras. Via topological $K$-theory we will use Theorem 2.3.1 to show that $\mathcal{H}(\mathcal{R}, q) \rtimes \Gamma$ and $\mathcal{S}(\mathcal{R}, q) \rtimes \Gamma$ have the same periodic cyclic homology, and that it does not depend on the (positive) parameter function $q$. The material in this section can be compared with BHP, Sol4].

Recall that the Chern character is a natural transformation $K_{*} \rightarrow H P_{*}$, where we write $H P_{*}(A)=H P_{0}(A) \oplus H P_{1}(A)$. By (5.1) and [Sol1, Theorem 6] there are natural isomorphisms

$$
K_{*}\left(C^{*}(\mathcal{R}, q) \rtimes \Gamma\right) \otimes_{\mathbb{Z}} \mathbb{C} \leftarrow K_{*}(\mathcal{S}(\mathcal{R}, q) \rtimes \Gamma) \otimes_{\mathbb{Z}} \mathbb{C} \rightarrow H P_{*}(\mathcal{S}(\mathcal{R}, q)),
$$

the first one is induced by the embedding $\mathcal{S}(\mathcal{R}, q) \rtimes \Gamma \rightarrow C^{*}(\mathcal{R}, q) \rtimes \Gamma$, the second one by the Chern character. Here and elsewhere in this paper the periodic cyclic homology of topological algebras is always meant with respect to the completed projective tensor product. (One needs a tensor product to build the differential complex whose homology is $H P_{*}$.) By contrast, in the definition of the periodic cyclic homology of nontopological algebras we simply use the algebraic tensor product over $\mathbb{C}$.

Theorem 5.2.1. The inclusion $\mathcal{H}(\mathcal{R}, q) \rtimes \Gamma \rightarrow \mathcal{S}(\mathcal{R}, q) \rtimes \Gamma$ induces an isomorphism on periodic cyclic homology. 
Proof. In [Sol4, Theorem 3.3] the author proved the corresponding result for Hecke algebras of reductive $p$-adic groups. The proof from [Sol4] also applies in our setting, the important representation-theoretic ingredients being Theorem 3.2.2, Proposition 3.4.1 and Lemma 3.4.2. A sketch of this proof already appeared in [Sol2].

Corollary 5.2.2. There exists a natural commutative diagram

$$
\begin{gathered}
H P_{*}(\mathbb{C}[W] \rtimes \Gamma) \rightarrow H P_{*}(\mathcal{S}(W) \rtimes \Gamma) \leftarrow K_{*}(\mathcal{S}(W) \rtimes \Gamma) \rightarrow K_{*}\left(C^{*}(W) \rtimes \Gamma\right) \\
\downarrow \\
\downarrow H P_{*}\left(\zeta_{0}\right) \\
\downarrow P_{*}(\mathcal{H}(\mathcal{R}, q) \rtimes \Gamma) \rightarrow H P_{*}(\mathcal{S}(\mathcal{R}, q) \rtimes \Gamma) \leftarrow K_{*}(\mathcal{S}(\mathcal{R}, q) \rtimes \Gamma) \rightarrow K_{*}\left(C^{*}(\mathcal{R}, q) \rtimes \Gamma\right)
\end{gathered}
$$

After applying $\otimes_{\mathbb{Z}} \mathbb{C}$ to the $K$-groups, all of these maps are isomorphisms.

Proof. The horizontal maps are induced the inclusion maps

$$
\mathcal{H}(\mathcal{R}, q) \rtimes \Gamma \rightarrow \mathcal{S}(\mathcal{R}, q) \rtimes \Gamma \rightarrow C^{*}(\mathcal{R}, q) \rtimes \Gamma
$$

and by the Chern character $K_{*} \rightarrow H P_{*}$. The vertical maps (expect the leftmost one) are induced by the Fréchet algebra homomorphisms $\zeta_{0}$ from Theorem 4.4.2, According to (5.9) and Theorem 5.2.1 all the horizontal maps become isomorphisms after tensoring the $K$-groups with $\mathbb{C}$. By Lemma 5.1.2 the maps $K_{*}\left(\zeta_{0}\right)$ are natural, and by Theorem 5.1 .4 they become isomorphisms after applying $\otimes_{\mathbb{Z}} \mathbb{C}$. The diagram commutes by functoriality, so $H P_{*}\left(\zeta_{0}\right)$ is also a natural isomorphism. Finally, we define $H P_{*}(\mathbb{C}[W] \rtimes \Gamma) \rightarrow H P_{*}(\mathcal{H}(\mathcal{R}, q) \rtimes \Gamma)$ as the unique map that makes the entire diagram commute.

Remark 5.2.3. Whether the leftmost vertical map comes from a suitable algebra homomorphism $\mathbb{C}[W] \rtimes \Gamma \rightarrow \mathcal{H}(\mathcal{R}, q) \rtimes \Gamma$ is doubtful, no such homomorphism is known if $q \neq 1$.

Suppose that $X$ is the weight lattice of $R_{0}^{\vee}$, that $q \in \mathbb{C} \backslash\{0\}$ is any complex number which is not a root of unity, and that $q(s)=q$ for all $s \in S^{\text {aff }}$. In this setting an isomorphism $H P_{*}(\mathbb{C}[W]) \cong H P_{*}(\mathcal{H}(\mathcal{R}, q))$ was already constructed by Baum and Nistor [BaNi, Theorem 11]. Their proof makes essential use of the KazhdanLusztig classification [KaLu2, Theorem 7.12] of irreducible $\mathcal{H}(\mathcal{R}, q)$-representations, and of Lusztig's asymptotic Hecke algebra [Lus3, Lus4].

For graded Hecke algebras things are even better than in Corollary 5.2.2 in [Sol6, Theorem 3.4] it was proven that not only $H P_{*}(\mathbb{H}(\tilde{\mathcal{R}}, k) \rtimes \Gamma)$, but also the cyclic homology and the Hochschild homology of $\mathbb{H}(\tilde{\mathcal{R}}, k) \rtimes \Gamma$ are independent of $k$. Whether or not this can be transferred to $\mathcal{H}(\mathcal{R}, q)$ is unclear to the author. The point is that the comparison of $\mathbb{H}(\tilde{\mathcal{R}}, k) \rtimes \Gamma$ with $\mathcal{H}(\mathcal{R}, q) \rtimes \Gamma$ goes only via analytic localizations of these algebras. Since the effect of localization on the dual space is very easy, we can translate the comparison between localized Hecke algebras to a comparison between their dual spaces. By [Sol6, Theorem 4.5] the periodic cyclic homology of a finite type algebra essentially depends only on its dual space, so it is not surprising that the parameter independence of $H P_{*}$ can be transferred from graded Hecke algebras to affine Hecke algebras,

On the other hand, the Hochschild homology of an algebra changes in a nontrivial way under localization. Therefore one would in the first instance only find a comparison between the Hochschild homology of two localized affine Hecke algebras with the same root datum but different parameters $q$. Possibly, provided that one would know enough about $H H_{*}(\mathcal{H}(\mathcal{R}, q) \rtimes \Gamma)$, one could deduce that also this vector space is independent of $q$. We remark that certainly the $Z(\mathcal{H}(\mathcal{R}, q) \rtimes \Gamma)$-module 
structure of $H H_{*}(\mathcal{H}(\mathcal{R}, q) \rtimes \Gamma)$ will depend on $q$, because that is already the case for graded Hecke algebras, see the remark to Theorem 3.4 in [Sol6].

5.3. Weakly spectrum preserving morphisms. For the statement and the proof of the Aubert-Baum-Plymen conjecture we need spectrum preserving morphisms and relaxed versions of those. These notions were developed in [BaNi, $\mathrm{Nis}$. Baum and Nistor work in the category of finite type $\mathbf{k}$-algebras, where $\mathbf{k}$ is the coordinate ring of some complex affine variety. Since we are also interested in certain Fréchet algebras, we work in a larger class of algebras.

We cannot do without some finiteness assumptions, but it suffices to impose them on representations. So, throughout this section we assume that for all our complex algebras $A$ there exists a $N \in \mathbb{N}$ such that the dimensions of irreducible $A$-modules are uniformly bounded by $N$. In particular, $\pi \mapsto \operatorname{ker} \pi$ is a bijection from $\operatorname{Irr}(A)$ to the collection of primitive ideals of $A$. A homomorphism $\phi: A \rightarrow B$ between two such algebras is called spectrum preserving if

- for every primitive ideal $J \subset B$, there is exactly one primitive ideal $I \subset A$ containing $\phi^{-1}(J)$;

- the map $J \mapsto I$ induces a bijection $\operatorname{Irr}(\phi): \operatorname{Irr}(B) \rightarrow \operatorname{Irr}(A)$.

We can relax these conditions in the following way. Suppose that there exists filtrations

$$
\begin{array}{lllllll}
A=A_{0} & \supset & A_{1} & \supset & \cdots & \supset & A_{n}=0, \\
B=B_{0} & \supset & B_{1} & \supset & \cdots & \supset & B_{n}=0
\end{array}
$$

by two sided ideals, such that $\phi\left(A_{i}\right) \subset B_{i}$ for all $i$. We call $\phi: A \rightarrow B$ weakly spectrum preserving if all the induced maps $\phi_{i}: A_{i} / A_{i+1} \rightarrow B_{i} / B_{i+1}$ are spectrum preserving. In this case there are bijections

$$
\begin{aligned}
& \bigsqcup_{i} \operatorname{Irr}\left(A_{i} / A_{i+1}\right) \rightarrow \operatorname{Irr}(A), \\
& \bigsqcup_{i} \operatorname{Irr}\left(B_{i} / B_{i+1}\right) \rightarrow \operatorname{Irr}(B), \\
& \operatorname{Irr}(\phi):=\sqcup_{i} \operatorname{Irr}\left(\phi_{i}\right): \operatorname{Irr}(B) \rightarrow \operatorname{Irr}(A) .
\end{aligned}
$$

Notice that $\operatorname{Irr}(\phi)$ depends not only on $\phi$, but also on the filtrations of $A$ and $B$.

Lemma 5.3.1. Let $\phi: A \rightarrow B$ be a weakly spectrum preserving morphism, and suppose that the dimensions of irreducible $B$-modules are uniformly bounded by $N \in \mathbb{N}$. Then $\operatorname{Irr}(\phi)^{-1}(V(I))=V\left(\phi(I)^{N}\right)$ for every two-sided ideal $I \subset A$. In particular, the bijection $\operatorname{Irr}(\phi)$ is continuous with respect to the Jacobson topology; cf. Section 3.4 .

Proof. We proceed with induction to the length $n$ of the filtration. For $n=1$ the morphism $\phi$ is spectrum preserving, so the statement reduces to [BaNi, Lemma 9]. For $n>1$ the induction hypothesis applies to the homomorphisms $\phi: A_{1} \rightarrow B_{1}$ and $\phi_{1}: A / A_{1} \rightarrow B / B_{1}$. So for $\pi \in \operatorname{Irr}\left(B / B_{1}\right) \subset \operatorname{Irr}(B)$ we have

$$
\begin{aligned}
& \pi \in \operatorname{Irr}(\phi)^{-1}(V(I)) \subset \operatorname{Irr}(B) \\
& \Longleftrightarrow \pi \in \operatorname{Irr}\left(\phi_{1}\right)^{-1}\left(V\left(I+A_{1} / A_{1}\right)\right) \subset \operatorname{Irr}\left(B / B_{1}\right) \\
&\left.\Longleftrightarrow \pi \in V\left(\phi(I)^{N}+B_{1} / B_{1}\right)\right) \subset \operatorname{Irr}\left(B / B_{1}\right) \\
&\left.\Longleftrightarrow \pi \in V\left(\phi(I)^{N}\right)\right) \subset \operatorname{Irr}(B) .
\end{aligned}
$$


A similar argument applies to $\pi \in \operatorname{Irr}\left(B_{1}\right) \subset \operatorname{Irr}(B)$.

The automatic continuity of $\operatorname{Irr}(\phi)$ enables us to extract a useful map from the Fréchet algebra morphism $\zeta_{0}$ :

Lemma 5.3.2. The morphism $\zeta_{0}: \mathcal{S}(W) \rtimes \Gamma \rightarrow \mathcal{S}(\mathcal{R}, q) \rtimes \Gamma$ is weakly spectrum preserving.

Proof. We wil make use of the proofs of Lemma 5.1 .3 and Theorem 5.1.4 There we constructed a $W_{0}^{\prime}$-equivariant triangulation of $T_{u n}$, which lead to two-sided ideals

$$
\begin{aligned}
I_{n}=C_{0}^{\infty}\left(\Sigma, \Sigma^{n-1}\right)^{W_{0}^{\prime}} & \subset C^{\infty}\left(T_{u n}\right)^{W_{0}^{\prime},}, \\
I_{n} \mathcal{S}(\mathcal{R}, q) \rtimes \Gamma & \subset \mathcal{S}(\mathcal{R}, q) \rtimes \Gamma, \\
I_{n} \mathcal{S}(W) \rtimes \Gamma & \subset \mathcal{S}(W) \rtimes \Gamma .
\end{aligned}
$$

(Here and below we regard the $n$-skeleton $\Sigma^{n}$ both as a simplicial complex and as a subset of $\left.T_{u n}\right)$. It suffices to show that the induced map

$$
\zeta_{0, n}: I_{n} \mathcal{S}(W) \rtimes \Gamma / I_{n+1} \mathcal{S}(W) \rtimes \Gamma \rightarrow I_{n} \mathcal{S}(\mathcal{R}, q) \rtimes \Gamma / I_{n+1} \mathcal{S}(\mathcal{R}, q) \rtimes \Gamma
$$

is spectrum preserving, for every $n$. Fortunately, the dual spaces of these quotient algebras are rather simple, by (5.3)

$$
\begin{aligned}
& \operatorname{Irr}\left(I_{n} \mathcal{S}(\mathcal{R}, q) \rtimes \Gamma / I_{n+1} \mathcal{S}(\mathcal{R}, q) \rtimes \Gamma\right) \\
& \quad \cong \bigsqcup_{\sigma \in \Sigma / W_{0}^{\prime}, \operatorname{dim} \sigma=n}(\sigma \backslash \delta \sigma) \times \operatorname{Irr}_{x_{\sigma}}(\mathcal{S}(\mathcal{R}, q) \rtimes \Gamma),
\end{aligned}
$$

where $x_{\sigma} \in \sigma \backslash \delta \sigma$ and $\operatorname{Irr}_{x_{\sigma}}(\mathcal{S}(\mathcal{R}, q) \rtimes \Gamma)$ denotes the dual space of the algebra

$$
\bigoplus_{\mathcal{G} \xi \in \Xi_{u n} / \mathcal{G}, \operatorname{pr}(\xi)=W_{0}^{\prime} x_{\sigma}} \mathcal{S}(\mathcal{R}, q) \rtimes \Gamma / \operatorname{ker} \pi^{\Gamma}(\xi) .
$$

By construction $\zeta_{0, n}$ is $C_{0}^{\infty}$-linear, so in particular it is linear over

$$
C_{0}^{\infty}\left(\Sigma^{n}, \Sigma^{n-1}\right):=I_{n} / I_{n+1}
$$

We know from Theorem 2.3.1. a and Corollary 4.4 .3 that $G_{\mathbb{Q}}\left(\zeta_{0, n}\right)$ is a bijection, so in particular

$$
G_{Q}\left(\zeta_{0, n}\right): G_{\mathbb{Q}}\left(\operatorname{Mod}_{x_{\sigma}}(\mathcal{S}(\mathcal{R}, q) \rtimes \Gamma)\right) \rightarrow G_{\mathbb{Q}}\left(\operatorname{Mod}_{x_{\sigma}}(\mathcal{S}(W) \rtimes \Gamma)\right)
$$

is a bijection. Any ordering $\left(\pi_{1}, \pi_{2}, \ldots, \pi_{k}\right)$ of $\operatorname{Irr}_{x_{\sigma}}(\mathcal{S}(\mathcal{R}, q) \rtimes \Gamma)$ gives rise to a filtration of (5.13) by ideals

$$
B_{i}:=\bigcap_{j=1}^{i} \operatorname{ker} \pi_{j} \quad i=0,1, \ldots, k .
$$

Since we are dealing with two finite dimensional semisimple algebras of the same rank $k$, (5.14) can be described completely with a matrix $M \in G L_{k}(\mathbb{Z})$. Order $\operatorname{Irr}_{x_{\sigma}}(\mathcal{S}(\mathcal{R}, q) \rtimes \Gamma)$ and $\operatorname{Irr}_{x_{\sigma}}(\mathcal{S}(W) \rtimes \Gamma)$ such that all the principal minors of $M$ are nonsingular. Then the corresponding ideals $B_{i}$ of (5.13) and $A_{i} \subset$ $\mathcal{S}(W) \rtimes \Gamma / \operatorname{ker} I_{x_{\sigma}}$ are such that $\zeta_{0, n}\left(x_{\sigma}\right)$ induces spectrum preserving morphisms $A_{i} / A_{i+1} \rightarrow B_{i} / B_{i+1}$. Hence $\zeta_{0, n}\left(x_{\sigma}\right)$ is weakly spectrum preserving.

It follows from this and (5.12) that for any $n$-dimensional simplex $\sigma \in \Sigma / W_{0}^{\prime}$ we can construct filtrations by two-sided ideals in

$$
C_{0}^{\infty}(\Sigma, \delta \sigma)^{W_{0}^{\prime}} \mathcal{S}(\mathcal{R}, q) \rtimes \Gamma / C_{0}^{\infty}(\Sigma, \sigma)^{W_{0}^{\prime}} \mathcal{S}(\mathcal{R}, q) \rtimes \Gamma
$$

and in

$$
C_{0}^{\infty}(\Sigma, \delta \sigma)^{W_{0}^{\prime}} \mathcal{S}(W) \rtimes \Gamma / C_{0}^{\infty}(\Sigma, \sigma)^{W_{0}^{\prime}} \mathcal{S}(W) \rtimes \Gamma
$$


with respect to which the map induced by $\zeta_{0, n}$ is weakly spectrum preserving. We do this for all such simplices $\sigma$, and then (5.3) shows that (5.11) is weakly spectrum preserving.

A related notion that we will use in the next section is geometric equivalence of algebras, as defined in ABP1, Section 4]. The basic idea is to call $A$ and $B$ geometrically equivalent if they are Morita equivalent or if there exists a weakly spectrum preserving morphism $\phi: A \rightarrow B$. Furthermore, two finite type k-algebras are geometrically equivalent if they only differ by an algebraic deformation of the k-module structure. Now one defines geometric equivalence to be the equivalence relation (on the category of finite type $\mathbf{k}$-algebras) generated by these three elementary moves.

So whenever two algebras $A$ and $B$ are geometrically equivalent, they are so by various sequences of elementary moves. Every such sequence induces a bijection between the dual spaces of $A$ and $B$, which, however, need not be continuous, since the map $\operatorname{Irr}(\phi)$ from Lemma 5.3.1 is usually not a homeomorphism. Nevertheless, by [BaNi, Theorem 8] every weakly spectrum preserving morphism of finite type algebras $\phi: A \rightarrow B$ induces an isomorphism $H P_{*}(\phi): H P_{*}(A) \rightarrow H P_{*}(B)$. The other two moves are easily seen to respect periodic cyclic homology, so geometric equivalence implies $H P$-equivalence.

5.4. The Aubert-Baum-Plymen conjecture. In a series of papers ABP1, ABP2, ABP3, ABP4 Aubert, Baum and Plymen developed a conjecture that describes the structure of Bernstein components in the smooth dual of a reductive $p$-adic group. We will rephrase this conjecture for affine Hecke algebras, and prove it in that setting.

A central role is played by extended quotients. Let $G$ be a finite group acting continuously on a topological space $T$. We endow

$$
\widetilde{T}:=\{(g, t) \in G \times T: g \cdot t=t\}
$$

with the subspace topology from $G \times T$. Then $G$ also acts continuously on $\widetilde{T}$, by

$$
g \cdot\left(g^{\prime}, t\right)=\left(g g^{\prime} g^{-1}, g \cdot t\right) .
$$

The extended quotient of $T$ by $G$ is defined as $\widetilde{T} / G$. It comes with a projection onto the normal quotient:

$$
\widetilde{T} / G \rightarrow T / G: G(g, t) \mapsto G t .
$$

The fiber over $G t \in T / G$ can be identified with the collection $\left\langle G_{t}\right\rangle$ of conjugacy classes in the isotropy group $G_{t}$.

The relevance of the extended quotient for representation theory comes from crossed product algebras. Suppose that $F(T)$ is an algebra of continuous complex valued functions on $T$, which separates the points of $T$ and is stable under the action of $G$ on $C(T)$. These conditions ensure that the crossed product $F(T) \rtimes G$ is well defined. The dual space of this algebra was determined in classical results that go back to Frobenius and Clifford (see [CuRe, Section 49]). Recall that a $F(T)$-weight of a representation $(\pi, V)$ is an element $t \in T$ such that there exists a $v \in V \backslash\{0\}$ with $\pi(f) v=f(t) v$ for all $f \in F(T)$. The collection of irreducible representations with a $F(T)$-weight $t \in T$ is in natural bijection with $\operatorname{Irr}\left(G_{t}\right)$, via the map

$$
\pi \mapsto \operatorname{Ind}_{F(T) \rtimes G_{t}}^{F(T) \rtimes G} \mathbb{C}_{t} \otimes \pi .
$$


Since $\left|\operatorname{Irr}\left(G_{t}\right)\right|=\left|\left\langle G_{t}\right\rangle\right|$, there exists a bijection

$$
\widetilde{T} / G \rightarrow \operatorname{Irr}(F(T) \rtimes G)
$$

which maps $G(g, t)$ to representation with a $F(T)$-weight $t$. With a little more work one can find a continuous bijection. However, it is not natural and a not a homeomorphism, except in very simple cases.

We return to an extended affine Hecke algebra $\mathcal{H}(\mathcal{R}, q) \rtimes \Gamma$. As described in Section 1.2, the parameter function $q$ is completely determined by its values on the quotient set $R_{n r}^{\vee} / W_{0} \rtimes \Gamma$. Let $\mathcal{Q}(\mathcal{R})$ be the complex variety of all maps $R_{n r}^{\vee} / W_{0} \rtimes$ $\Gamma \rightarrow \mathbb{C}^{\times}$. To every $v \in \mathcal{Q}(\mathcal{R})$ we associate the parameter function $q_{\alpha^{\vee}}=v\left(\alpha^{\vee}\right)^{2}$.

Conjecture 5.4.1 (ABP-conjecture for affine Hecke algebras). Suppose that the subgroup of $\mathbb{C}^{\times}$generated by $\left\{q(s)^{ \pm 1 / 2}\right\}$ contains no roots of unity (except 1 ).

(a) The algebras $\mathbb{C}[W] \rtimes \Gamma$ and $\mathcal{H}(\mathcal{R}, q) \rtimes \Gamma$ are geometrically equivalent.

(b) There exists a canonical isomorphism $H P_{*}(\mathbb{C}[W] \rtimes \Gamma) \cong H P_{*}(\mathcal{H}(\mathcal{R}, q) \rtimes \Gamma)$.

(c) There exists a continuous bijection $\mu: \widetilde{T} / W_{0}^{\prime} \rightarrow \operatorname{Irr}(\mathcal{H}(\mathcal{R}, q) \rtimes \Gamma)$ such that $\mu\left(\widetilde{T_{u n}} / W_{0}^{\prime}\right)=\operatorname{Irr}(\mathcal{S}(\mathcal{R}, q) \rtimes \Gamma)$.

(d) For every connected component $c$ of $\widetilde{T} / W_{0}^{\prime}$ there exists a smooth morphism of algebraic varieties $h_{c}: \mathcal{Q}(\mathcal{R}) \rightarrow T$ with the following properties.

For all components $c$ we have $h_{c}(1)=1$, and

$$
\operatorname{pr}_{q^{1 / 2}}\left(\widetilde{T} / W_{0}^{\prime}-T / W_{0}^{\prime}\right)=\{t \in T: M(t) \text { is reducible }\} / W_{0}^{\prime},
$$

where $\operatorname{pr}_{v}: \widetilde{T} / W_{0}^{\prime} \rightarrow T / W_{0}^{\prime}$ is defined by

$$
\operatorname{pr}_{v}\left(W_{0}^{\prime}(w, t)\right)=W_{0}^{\prime} h_{c}(v) t \quad \text { for } \quad v \in \mathcal{Q}(\mathcal{R}), W_{0}^{\prime}(w, t) \in c .
$$

Moreover, $\mu$ can be chosen such that the central character of $\mu\left(W_{0}^{\prime}(w, t)\right)$ is $W_{0}^{\prime} h_{c}\left(q^{1 / 2}\right) t$ for $W_{0}^{\prime}(w, t) \in c$.

The assumption on the roots of unity is probably stronger than necessary, but it is difficult to predict which roots of unity really cause problems. In any case, it is known that the above statements are false for some specific of roots unity, for example, $q=-1$ if $\mathcal{R}$ is of type $A_{1}^{(1)}$.

We will verify the ABP-conjecture in the following cases:

Theorem 5.4.2. Parts (b), (c) and (d) of Conjecture 5.4.1 hold for every extended affine Hecke algebra with a positive parameter function q. Part (a) holds for the Schwartz completions of the algebras in question.

Let us discuss the different parts of the ABP-conjecture. As we mentioned at the end of Section 5.3. every explicit geometric equivalence gives rise to an isomorphism on periodic cyclic homology. However, this isomorphism need not be natural, so (b) does not yet follow from (a). In [ABP1, ABP3] we see that Aubert, Baum and Plymen have a geometric equivalence via Lusztig's asymptotic Hecke algebra Lus4] in mind. The corresponding isomorphism [BaNi, Theorem 11]

$$
H P_{*}(\mathcal{H}(\mathcal{R}, q)) \cong H P_{*}(\mathbb{C}[W])
$$

can be regarded as canonical, albeit in a rather weak sense.

Unfortunately, for unequal parameter functions this asymptotic Hecke algebra exists only as a conjecture; see [Lus8, Section 18]. The author does not know any other way to construct a geometric equivalence between $\mathcal{H}(\mathcal{R}, q) \rtimes \Gamma$ and $\mathbb{C}[W] \rtimes \Gamma$, so this part of the conjecture remains open for unequal parameter functions. As a 
substitute we offer Lemma 5.3.2 which has approximately the same strength. It is weaker because it concerns only topologically completed versions of the algebras, but it is stronger in the sense that the geometric equivalence consists of only one weakly spectrum preserving morphism.

Part (b) of Conjecture 5.4.1 was already dealt with in Corollary 5.2.2.

Proof of Conjecture 5.4.1. c. Recall the definition of a (tempered) smooth family of $\mathcal{H} \rtimes \Gamma$-representations from (3.30). By (3.39) there exist tempered smooth families $\left\{\pi_{i, t}: t \in V_{i}\right\}$ which together form a basis of $G_{\mathbb{Q}}(\mathcal{S}(\mathcal{R}, q) \rtimes \Gamma)$. The parameter space of such a family is of the form $V_{i}=u_{i} \exp \left(\mathfrak{a}^{g_{i}}\right)$ for some $u_{i} \in T_{u n}, g_{i} \in W_{0}^{\prime}$. By (3.36) the number of families with parameter space of the form $g V_{i}$ for some $g \in W_{0}^{\prime}$, is precisely the number of components of $\widetilde{T_{u n}} / W_{0}^{\prime}$ whose projection onto $T_{u n} / W_{0}^{\prime}$ is $W_{0}^{\prime} V_{i}$. Hence we can find a continuous bijection

$$
\widetilde{T_{u n}} / W_{0}^{\prime} \rightarrow\left\{\pi_{i, t}: t \in V_{i}, i \in I\right\} .
$$

This will be our map $\mu$ whenever the image $\pi_{i, t}$ is irreducible, which is the case on a Zariski-dense subset of $\widetilde{T_{u n}} / W_{0}^{\prime}$. To extend $\mu$ continuously to all nongeneric points, we need to find irreducible subrepresentations $\pi_{i, t}^{\prime} \subset \pi_{i, t}$, such that $\left\{\pi_{i, t}^{\prime}\right.$ : $\left.t \in V_{i}\right\}=\operatorname{Irr}(\mathcal{S}(\mathcal{R}, q) \rtimes \Gamma)$. For 0-dimensional components all points are generic, so there is nothing to do. If we have already defined $\mu$ on all components of $\widetilde{T_{u n}} / W_{0}^{\prime}$ of dimension smaller than $d$, and $(i, t)$ corresponds to a nongeneric point $W_{0}^{\prime}(w, t)$ in a component of dimension $d$, than we choose for $\mu\left(W_{0}^{\prime}(w, t)\right)$ any irreducible subrepresentation of $\pi_{i, t}$ that we did not have yet in the image of the previously handled components. This process can be carried out completely (3.36), and yields a continuous bijection

$$
\mu: \widetilde{T_{u n}} / W_{0}^{\prime} \rightarrow \operatorname{Irr}(\mathcal{S}(\mathcal{R}, q) \rtimes \Gamma) .
$$

From this and Lemmas 5.3 .1 and 5.3 .2 we obtain continuous bijections

$$
\widetilde{T_{\text {un }}} / W_{0}^{\prime} \rightarrow \operatorname{Irr}(\mathcal{S}(\mathcal{R}, q) \rtimes \Gamma) \rightarrow \operatorname{Irr}(\mathcal{S}(W) \rtimes \Gamma) .
$$

As explained after (3.29) and (3.39), these can be extended canonically to continuous bijections

$$
\widetilde{T} / W_{0}^{\prime} \rightarrow \operatorname{Irr}(\mathcal{H}(\mathcal{R}, q) \rtimes \Gamma) \rightarrow \operatorname{Irr}(W \rtimes \Gamma) .
$$

Proof of Conjecture 5.4.1. $d$. Suppose that $W_{0}^{\prime}\left(w, t_{0}\right) \in \widetilde{T_{u n}} / W_{0}^{\prime}$ is such that the corresponding representation $\pi_{i, t}$ is irreducible. With Theorem 3.3.2 we can find an induction datum $\xi^{+}\left(\pi_{i, t}\right)=\left(P, \delta, t_{1}\right) \in \Xi_{u n}$ such that $\pi_{i, t}$ is a subquotient of $\pi^{\Gamma}\left(P, \delta, t_{1}\right)$. Then $\mu\left(W_{0}^{\prime}\left(w, t_{0} t_{2}\right)\right)$ is a subquotient of $\pi^{\Gamma}\left(P, \delta, t_{1} t_{2}\right)$ for all $t_{2} \in$ $\exp \left(\mathfrak{t}^{w}\right)$, so its central character is $W_{0}^{\prime} r t_{1} t_{2}$, where $W\left(R_{P}\right) r \in T_{P} / W\left(R_{P}\right)$ is the central character of the $\mathcal{H}_{P}$-representation $\delta$. According to Opd2, Lemma 3.31] $r \in T_{P}$ is a residual point for $\mathcal{R}_{P}$, which by Proposition 2.63 and Theorem 2.58 of OpSo2 means that the coordinates of $r$ can be expressed as an element of $T_{P, \text { un }}$ times a monomial in the variables $\left\{q(s)^{ \pm 1 / 2}: s \in S_{\text {aff }}\right\}$. Hence we can write $|r|=h_{c}\left(q^{1 / 2}\right)$, where $c$ is the component of $\widetilde{T} / W_{0}^{\prime}$ containing $W_{0}^{\prime}(w, t)$ and $h_{c}: \mathcal{Q}(\mathcal{R}) \rightarrow T_{P} \subset T$ is a smooth algebraic morphism with $h_{c}(1)=1$. Now $W_{0}^{\prime} h_{c}\left(q^{1 / 2}\right) t_{0} t_{2}$ is by construction the central character of $\mu\left(W_{0}^{\prime}\left(w, t_{0} t_{2}\right)\right)$. We note that the discrete series representation $\delta_{\emptyset}$ of $\mathcal{H}_{\emptyset}=\mathbb{C}$ has central character $1 \in T_{\emptyset}=$ $\{1\}$, so $h_{c}=1$ when $c$ has dimension $\operatorname{rank}(X)$. 
Let $\mathrm{pr}_{q^{1 / 2}}$ be as in part (d) of Conjecture 5.4.1 and temporarily denote the difference of two sets by - . Then $\operatorname{pr}_{q^{1 / 2}}\left(\widetilde{T} / W_{0}^{\prime}-T / W_{0}^{\prime}\right)$ is the set of central characters of $\mu\left(\widetilde{T} / W_{0}^{\prime}-T / W_{0}^{\prime}\right)$. Since $\mu$ parametrizes irreducible representations, and since every $\pi \in \operatorname{Irr}(\mathcal{H}(\mathcal{R}, q) \rtimes \Gamma)$ with central character $t$ is a quotient of the principal series representation $M(t)$, no element of $\operatorname{pr}_{q^{1 / 2}}\left(\widetilde{T} / W_{0}^{\prime}-T / W_{0}^{\prime}\right)$ can be the parameter of an irreducible principal series representation.

Conversely, suppose that $t \in T$ is not in the aforementioned set. In view of Lemma 3.1.7 we may assume that $t \in T^{+}$. Then there is, up to isomorphism, only one $\pi_{t} \in \operatorname{Irr}(\mathcal{H}(\mathcal{R}, q) \rtimes \Gamma)$ with central character $W_{0}^{\prime} t$. In particular, all constituents of $M(t)$ are isomorphic to $\pi_{t}$. Restricted to the finite dimensional semisimple algebra $\mathcal{H}\left(W_{0}, q\right) \rtimes \Gamma$ this means that the regular representation $M(t)$ of $\mathcal{H}\left(W_{0}, q\right) \rtimes$

$\Gamma$ is a direct sum of copies of $\left.\pi_{t}\right|_{\mathcal{H}\left(W_{0}, q\right) \rtimes \Gamma}$. But $\mathcal{H}\left(W_{0}, q\right) \rtimes \Gamma$ has irreducible representations that appear only once in the regular representation, for example, the trivial one-dimensional representation $N_{w} \gamma \mapsto q(w)^{1 / 2}$. Thus there can be only one copy of $\pi_{t}$ involved and

$$
\pi_{t} \cong \mathcal{H}\left(W_{0}, q\right) \rtimes \Gamma \cong M(t)
$$

as representations of $\mathcal{H}\left(W_{0}, q\right) \rtimes \Gamma$. Moreover, $\pi_{t}$ is a subquotient of $M(t)$ and both have finite dimension, so they are also isomorphic as $\mathcal{H} \rtimes \Gamma$-representations. Therefore $\operatorname{pr}_{q^{1 / 2}}\left(\widetilde{T} / W_{0}^{\prime}-T / W_{0}^{\prime}\right)$ is precisely the subset of $t \in T$ for which the principal series representation $M(t)$ is reducible.

By (3.7) $\operatorname{pr}_{q^{1 / 2}}\left(\widetilde{T} / W_{0}^{\prime}-T / W_{0}^{\prime}\right)$ contains all residual cosets of dimension smaller than $\operatorname{dim}_{\mathbb{C}}(T)$. However, in general it is larger, because a unitary principal series representation can be reducible. In fact, there is also a more direct criterium for irreducibility of principal series representations, in terms of the functions $c_{\alpha}$ Kat1, Theorem 2.2].

We note that by Proposition 4.1 .2 the same map $\operatorname{pr}_{v}$ also makes part (d) valid for the scaled parameter functions $q^{\epsilon}$ with $\epsilon \in \mathbb{R}$. However, for other parameter functions changes can occur.

Theorem 5.4 .2 also proves the Aubert-Baum-Plymen conjecture for many Bernstein components of reductive $p$-adic groups:

Corollary 5.4.3. Let $\mathfrak{s}$ be a Bernstein component of a reductive p-adic group $G$ such that the algebra $\mathcal{H}(G)_{\mathfrak{s}}$ is Morita equivalent to an extended affine Hecke algebra $\mathcal{H}(\mathcal{R}, q) \rtimes \Gamma$ in the way described in Section 1.6. (In particular, this applies to all Bernstein components listed at the end of that section.)

The part of the Aubert-Baum-Plymen conjecture ABP1, ABP2] corresponding to parts (b), (c) and (d) of Conjecture 5.4.1 holds for $\mathfrak{s}$. If, moreover, $\mathcal{S}(G)_{\mathfrak{s}}$ is Morita equivalent to $\mathcal{S}(\mathcal{R}, q) \rtimes \Gamma$, then it is geometrically equivalent to $\mathcal{S}(\mathcal{R}, 1) \rtimes \Gamma$.

Proof. Let us write $\mathfrak{s}=[M, \sigma]_{G}$. As discussed in (1.28), the assumed Morita equivalence between $\mathcal{H}(G)_{\mathfrak{s}}$ and $\mathcal{H}(\mathcal{R}, q) \rtimes \Gamma$ comes from an isomorphism between $(\mathcal{R}, \Gamma)$ and $\left(\mathcal{R}_{\sigma}, \Gamma_{\sigma}\right)$, the latter being naturally associated to $(M, \sigma)$. These yield isomorphisms $W_{\sigma} \rightarrow W_{0} \rtimes \Gamma$ and $X_{u r}\left(M_{\sigma}\right) \rightarrow T$, such that the latter is equivariant with respect to the former and restricts to bijections between the unitary and the positive subsets of $X_{u r}\left(M_{\sigma}\right)$ and $T$, see (3.19). Moreover, we obtain an isomorphism between $\mathcal{Q}(\mathcal{R})$ and the variety of parameter functions for $\mathcal{R}_{\sigma}$.

With these correspondences at hand, Theorem 5.4 .2 this proves parts (b), (c) and (d) of Conjecture 5.4 .1 for $\mathcal{H}(G)_{\mathfrak{s}}$. As discussed after (3.19), it is not unlikely 
that $\mathcal{S}(G)_{\mathfrak{s}}$ is Morita equivalent to $\mathcal{S}(\mathcal{R}, q) \rtimes \Gamma$. In that case Lemma 5.3 .2 provides the required geometric equivalence.

5.5. Example: type $C_{2}^{(1)}$. In the final section we illustrate what the AubertBaum-Plymen conjecture looks like for an affine Hecke algebra with $R_{0}$ of type $B_{2} / C_{2}$ and $X$ the root lattice. More general results for type $C_{n}^{(1)}$ affine Hecke algebras can be found in [Kat2, CiKa]. For other examples we refer to Sol3, Section 6].

Consider the based root datum $\mathcal{R}$ with

$$
\begin{aligned}
& X=Y=\mathbb{Z}, \\
& R_{0}=\{x \in X:\|x\|=1 \text { or }\|x\|=\sqrt{2}\}, \\
& R_{0}^{\vee}=\{y \in Y:\|x\|=2 \text { or }\|x\|=\sqrt{2}\}, \\
& F_{0}=\left\{\alpha_{1}=\left(\begin{array}{c}
1 \\
-1
\end{array}\right), \alpha_{2}=\left(\begin{array}{l}
0 \\
1
\end{array}\right)\right\} .
\end{aligned}
$$

Then $\alpha_{4}=\left(\begin{array}{c}1 \\ 1\end{array}\right)$ is the longest root and $\alpha_{3}^{\vee}=\left(\begin{array}{l}2 \\ 0\end{array}\right)$ is the longest coroot, so

$$
S_{\text {aff }}=\left\{s_{\alpha_{1}}, s_{\alpha_{2}}, t_{\alpha_{3}} s_{\alpha_{3}}\right\} \text {. }
$$

We write $s_{i}=s_{\alpha_{i}}$ for $1 \leq i \leq 4$ and $s_{0}=t_{\alpha_{3}} s_{\alpha_{3}}$. The Weyl group $W_{0}$ is isomorphic to $D_{4}$ and consists of the elements

$$
W_{0}=\left\{e, \rho_{\pi / 2}, \rho_{\pi}, \rho_{-\pi / 2}\right\} \cup\left\{s_{1}, s_{2}, s_{3}, s_{4}\right\},
$$

where $\rho_{\theta}$ denotes the rotation with angle $\theta$. The affine Weyl group of $\mathcal{R}$ is the Coxeter group

$$
W_{\text {aff }}=W=X \rtimes W_{0}=\left\langle s_{0}, s_{1}, s_{2} \mid s_{i}^{2}=\left(s_{0} s_{2}\right)^{2}=\left(s_{1} s_{2}\right)^{4}=\left(s_{0} s_{1}\right)^{4}=e\right\rangle .
$$

Furthermore, $R_{n r}=R_{0} \cup\left\{ \pm \alpha_{2}, \pm \alpha_{3}\right\}$ and $X^{+}=\left\{\left(\begin{array}{c}m \\ n\end{array}\right) \in X: m \geq n \geq 0\right\}$.

We note that $\mathcal{R}$ is the root datum of the algebraic group $S O_{5}$, while $\mathcal{R}^{\vee}$ corresponds to $S p_{4}$. Let $\mathbb{F}$ be a $p$-adic field whose residue field has $q$ elements, and let $\mathfrak{s}$ be the Iwahori-spherical component of $S p_{4}(\mathbb{F})$. Then $\operatorname{Mod}_{\mathfrak{s}}\left(S p_{4}(\mathbb{F})\right) \cong \operatorname{Mod}(\mathcal{H}(\mathcal{R}, q))$ and Kazhdan-Lusztig theory describes the irreducible representations in this category with data from $S p_{4}(\mathbb{F})$.

But there are many more parameter functions for $\mathcal{R}$. Since $s_{0}, s_{1}$ and $s_{2}$ are not conjugate in $W$, we can independently choose three parameters:

$$
q_{0}=q\left(s_{0}\right)=q_{\alpha_{2}^{\vee} / 2}, q_{1}=q\left(s_{1}\right)=q_{\alpha_{1}}, q_{2}=q\left(s_{2}\right)=q_{\alpha_{2}^{\vee}} .
$$

Several combinations of these parameters occur in Hecke algebras associated to nonsplit $p$-adic groups; see Lus7]. The $c$-functions are

$$
c_{\alpha_{1}}=\frac{\theta_{\alpha_{1}}-q_{1}^{-1}}{\theta_{\alpha_{1}}-1} \text { and } c_{\alpha_{2}}=\frac{\theta_{\alpha_{2}}+q_{2}^{-1 / 2} q_{0}^{1 / 2}}{\theta_{\alpha_{2}}+1} \frac{\theta_{\alpha_{2}}-q_{1}^{-1 / 2} q_{0}^{-1 / 2}}{\theta_{\alpha_{2}}-1} \text {. }
$$

For $q_{0}=q_{2}$ the relations from Theorem $1.2 .1 \mathrm{~d}$ simplify to

$$
f N_{s_{i}}=N_{s_{i}} s_{i}(f)=\left(q_{i}^{1 / 2}-q_{i}^{-1 / 2}\right)\left(f-s_{i}(f)\right)\left(1-\theta_{-\alpha_{i}}\right)^{-1} \quad i=1,2 .
$$

In contrast with graded Hecke algebras, $\mathcal{H}\left(\mathcal{R}=\mathcal{R}\left(S O_{5}\right), q_{1}, q_{2}=q_{0}\right)$ is not isomorphic to $\mathcal{H}\left(\mathcal{R}^{\vee}=\mathcal{R}\left(S p_{4}\right), q_{2}, q_{1}\right)$. The reason is that in $\mathcal{H}\left(\mathcal{R}^{\vee}, q_{2}, q_{1}\right)$ the relation

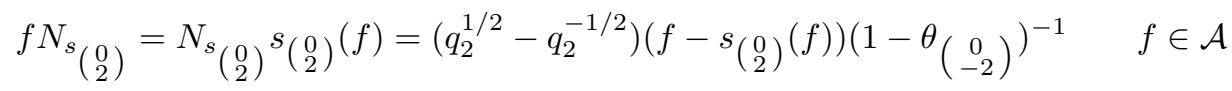

holds, which really differs from $(\underline{5.18})$ because the root lattice $\mathbb{Z}\left(\begin{array}{c}-1 \\ 1\end{array}\right)+\mathbb{Z}\left(\begin{array}{l}0 \\ 2\end{array}\right)$ does not equal $X$ for the root datum $\mathcal{R}^{\vee}$. 
We will work out the tempered dual of $\mathcal{H}(\mathcal{R}, q)$ for almost all positive parameter functions $q$. To this end we discuss for each parabolic subalgebra $\mathcal{H}_{P}$ with $P \subset$ $\left\{\alpha_{1}, \alpha_{2}\right\}$ separately. Its contribution to $\operatorname{Irr}(\mathcal{S}(\mathcal{R}, q))$ will of course depend on $q$, and can even be empty in some cases.

- $\quad P=\emptyset$

$$
\begin{aligned}
& X_{P}=\{0\}, X^{P}=X, Y_{P}=\{0\}, Y^{P}=Y, R_{P}=\emptyset, R_{P}^{\vee}=\emptyset, W\left(R_{P}\right)=\{e\}, \\
& T_{P}=\{1\}, T^{P}=T, \mathcal{G}_{P}=W_{0}, \mathcal{H}_{P}=\mathbb{C}, \mathcal{H}^{P}=\mathcal{A} \cong \mathbb{C}[X] .
\end{aligned}
$$

We must determine the reducibility of the unitary principal series representations

$$
M(t)=\operatorname{Ind}_{\mathcal{A}}^{\mathcal{H}} \mathbb{C}_{t}=\pi\left(\emptyset, \delta_{\emptyset}, t\right) \quad t \in T_{u n} .
$$

By Theorem 3.3.1 $\operatorname{End}_{\mathcal{H}}(M(t))$ is spanned by the intertwining operators $\pi\left(w, \emptyset, \delta_{\emptyset}, t\right)$ with $w \in W_{0}$ and $w(t)=t$. For a root $\alpha \in R_{0}$ with $s_{\alpha}(t)=t$, Lemma 3.1.6 tells us that $\pi\left(s_{\alpha}, \emptyset, \delta_{\emptyset}, t\right)$ is a scalar if and only if $c_{\alpha}^{-1}(t)=0$.

Let us write $t=\left(t_{3}, t_{2}\right)$ with $t_{i}=t\left(\alpha_{i}\right)$. A fundamental domain for the action of $W_{0}$ on $T_{u n}$ is $\left\{t=\left(e^{i \phi}, e^{i \psi}\right): 0 \leq \psi \leq \phi \leq \pi\right\}$ :

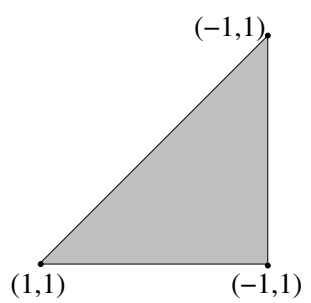

\begin{tabular}{|c|c|c|c|c|}
\hline$t$ & $W_{0, t}$ & conditions & $\operatorname{End}_{\mathcal{H}}(M(t))$ & \# irreducibles \\
\hline \multirow[t]{2}{*}{$\overline{\left(e^{i \phi}, 1\right), \phi \in(0, \pi)}$} & $\left\langle s_{2}\right\rangle$ & $q_{0} q_{2} \neq 1$ & $\mathbb{C}$ & 1 \\
\hline & & $q_{0} q_{2}=1$ & $\mathbb{C}\left[\left\langle s_{2}\right\rangle\right]$ & 2 \\
\hline \multirow[t]{2}{*}{$\left(-1, e^{i \psi}\right), \psi \in(0, \pi)$} & $\left\langle s_{3}\right\rangle$ & $q_{2} \neq q_{0}$ & $\mathbb{C}$ & 1 \\
\hline & & $q_{2}=q_{0}$ & $\mathbb{C}\left[\left\langle s_{3}\right\rangle\right]$ & 2 \\
\hline \multirow[t]{2}{*}{$\left(e^{i \phi}, e^{i \phi}\right), \phi \in(0, \pi)$} & $\left\langle s_{1}\right\rangle$ & $q_{1} \neq 1$ & $\mathbb{C}$ & 1 \\
\hline & & $q_{1}=1$ & $\mathbb{C}\left[\left\langle s_{1}\right\rangle\right]$ & 2 \\
\hline \multirow[t]{4}{*}{$(-1,1)$} & $\left\langle s_{2}, s_{3}\right\rangle$ & $q_{0} \neq q_{2}, q_{0} q_{2} \neq 1$ & $\mathbb{C}^{1}$ & 1 \\
\hline & & $q_{0}=q_{2} \neq 1$ & $\mathbb{C}\left[\left\langle s_{3}\right\rangle\right]$ & 2 \\
\hline & & $q_{0}=q_{2}^{-1} \neq 1$ & $\mathbb{C}\left[\left\langle s_{2}\right\rangle\right]$ & 2 \\
\hline & & $q_{0}=q_{2}=1$ & $\mathbb{C}\left[\left\langle s_{2}, s_{3}\right\rangle\right]$ & 4 \\
\hline \multirow[t]{4}{*}{$(-1,-1)$} & $W_{0}$ & $q_{0} \neq q_{2}, q_{1} \neq 1$ & $\mathbb{C}$ & 1 \\
\hline & & $q_{0}=q_{2}, q_{1} \neq 1$ & $\mathbb{C}\left[\left\langle s_{2}\right\rangle\right]$ & 2 \\
\hline & & $q_{1}=1, q_{0} \neq q_{2}$ & $\mathbb{C}\left[\left\langle s_{1}\right\rangle\right]$ & 2 \\
\hline & & $q_{0}=q_{2}, q_{1}=1$ & $\mathbb{C}\left[W_{0}\right]$ & 5 \\
\hline \multirow[t]{4}{*}{$(1,1)$} & $W_{0}$ & $q_{0} q_{2} \neq 1, q_{1} \neq 1$ & $\mathbb{C}$ & 1 \\
\hline & & $q_{0}=q_{2}^{-1}, q_{1} \neq 1$ & $\mathbb{C}\left[\left\langle s_{2}\right\rangle\right]$ & 2 \\
\hline & & $q_{1}=1, q_{0} \neq q_{2}^{-1}$ & $\mathbb{C}\left[\left\langle s_{3}\right\rangle\right]$ & 2 \\
\hline & & $q_{0}=q_{2}^{-1}, q_{1}=1$ & $\mathbb{C}\left[W_{0}\right]$ & 5 \\
\hline
\end{tabular}

The isotropy groups are trivial for all interior points, so $M(t)$ is irreducible for such $t$. Below we list the necessary data for all boundary points: 


$$
\begin{aligned}
& \text { - } \quad P=\left\{\alpha_{1}\right\} \\
& X_{P}=X / \mathbb{Z} \alpha_{4} \cong \mathbb{Z} \alpha_{1} / 2, X^{P}=X / \mathbb{Z} \alpha_{1}, Y_{P}=\mathbb{Z} \alpha_{1}^{\vee}, Y^{P}=\mathbb{Z} \alpha_{4}^{\vee}, R_{P}=\left\{ \pm \alpha_{1}\right\}, \\
& R_{P}^{\vee}=\left\{ \pm \alpha_{1}^{\vee}\right\}, W\left(R_{P}\right)=\left\{e, s_{1}\right\}, T^{P}=\left\{t \in T: t_{3}=t_{2}\right\}, T_{P}=\left\{t \in T: t_{2} t_{3}=1\right\}, \\
& T^{P} \cap T_{P}=\{(1,1),(-1,-1)\}, \mathcal{G}_{P}=\left\{e, s_{4}\right\} \times T^{P} \cap T_{P}, \\
& \mathcal{H}_{P}=\mathcal{H}\left(\mathcal{R}_{P}, q\left(s_{1}\right)=q_{1}=q_{\alpha_{1}^{\vee}}, \mathcal{H}^{P}=\mathcal{H}_{P} \ltimes \mathbb{C}\left[X^{P}\right] .\right.
\end{aligned}
$$

The root datum $\mathcal{R}_{P}$ is of type $C_{1}^{(1)}$, which means that $R_{0}^{\vee}$ is of type $C_{1}=A_{1}$ and generates the lattice $Y_{P}$. For $q_{1}=1$ there are no residual points, for $q_{1} \neq 1$ there are two orbits, namely $W\left(R_{P}\right)\left(q_{1}^{1 / 2}, q_{1}^{-1 / 2}\right)$ and $W\left(R_{P}\right)\left(-q_{1}^{1 / 2},-q_{1}^{-1 / 2}\right)$. Both orbits carry a unique discrete series representation, which has dimension one. The formulas for these representations are not difficult, but they depend on whether $q_{2}>1$ or $q_{2}<1$. So we obtain two families of $\mathcal{H}(\mathcal{R}, q)$-representations:

$$
\begin{array}{ll}
\pi\left(\left\{\alpha_{1}\right\}, \delta_{1},\left(t_{2}, t_{2}\right)\right)=\operatorname{Ind}_{\mathcal{H}^{P}}^{\mathcal{H}}\left(\delta_{1} \circ \phi_{\left(t_{2}, t_{2}\right)}\right) & q_{1} \neq 1, t_{2} \in S^{1}, \\
\pi\left(\left\{\alpha_{1}\right\}, \delta_{1}^{\prime},\left(t_{2}, t_{2}\right)\right)=\operatorname{Ind}_{\mathcal{H}^{P}}^{\mathcal{H}}\left(\delta_{1}^{\prime} \circ \phi_{\left(t_{2}, t_{2}\right)}\right) & q_{1} \neq 1, t_{2} \in S^{1} .
\end{array}
$$

The action of $\mathcal{G}_{P}$ on these families is such that $s_{4}\left(t_{2}, t_{2}\right)=\left(t_{2}^{-1}, t_{2}^{-1}\right)$, while $(-1,-1)$ $\in \mathcal{G}_{P}$ simultaneously exchanges $\delta_{1}$ with $\delta_{1}^{\prime}$ and $\left(t_{2}, t_{2}\right)$ with $\left(-t_{2},-t_{2}\right)$. A fundamental domain for this action is $\left\{\left(\left\{\alpha_{1}\right\}, \delta_{1},\left(e^{i \phi}, e^{i \phi}\right)\right): \phi \in[0, \pi]\right\}$. For $\phi \in(0, \pi)$ these points have trivial stabilizer in $\mathcal{G}_{P}$, so the corresponding $\mathcal{H}$-representations are irreducible. On the other hand, the element $s_{4} \in \mathcal{G}_{P}$ fixes the points with $\phi=0$ or $\phi=\pi$, so the representations $\pi\left(\left\{\alpha_{1}\right\}, \delta_{1},(1,1)\right)$ and $\pi\left(\left\{\alpha_{1}\right\}, \delta_{1},(-1,-1)\right)$ can be reducible. Whether or not this happens depends on more subtle relations between $q_{0}, q_{1}$ and $q_{2}$.

$$
\begin{aligned}
& P=\left\{\alpha_{2}\right\} \\
& X_{P}=X / \mathbb{Z} \alpha_{3} \cong \mathbb{Z} \alpha_{2}, X^{P}=X / \mathbb{Z} \alpha_{2} \cong \mathbb{Z} \alpha_{3}, Y_{P}=\mathbb{Z} \alpha_{1}^{\vee}, Y^{P}=\mathbb{Z} \alpha_{3}^{\vee} / 2, \\
& R_{0}=\left\{ \pm \alpha_{2}\right\}, R_{0}^{\vee}=\left\{ \pm \alpha_{2}^{\vee}\right\}, W\left(R_{P}\right)=\left\{e, s_{2}\right\}, \\
& T^{P}=\left\{t \in T: t_{2}=1\right\}, T_{P}=\left\{t \in T: t_{3}=1\right\}, \mathcal{G}_{P}=\left\{e, s_{3}\right\}, \\
& \mathcal{H}_{P} \cong \mathcal{H}\left(\mathcal{R}_{P}, q\left(s_{2}\right)=q_{2}, q_{\alpha_{2}^{\vee}}=q_{0}\right), \mathcal{H}^{P} \cong \mathcal{H}_{P} \otimes \mathbb{C}\left[X^{P}\right] .
\end{aligned}
$$

The root datum $\mathcal{R}_{P}$ is of type $A_{1}^{(1)}$, which differs from $C_{1}^{(1)}$ in the sense that $X_{P}$ is the root lattice. There are two orbits of residual points: $W\left(R_{P}\right)\left(1, q_{0}^{1 / 2} q_{2}^{1 / 2}\right)$ and $W\left(R_{P}\right)\left(1,-q_{0}^{1 / 2} q_{2}^{-1 / 2}\right)$. That is, these points are residual unless they equal $(1,1)$ or $(1,-1)$. Both orbits admit a unique discrete series representation, of dimension one, which denote by $\delta_{+}$or $\delta_{-}$. Like for $P=\left\{\alpha_{1}\right\}$, the explicit formulas depend on which of the $\mathcal{A}_{P}$-characters are in $T_{P}^{--}$. Again, we find two families of $\mathcal{H}$-representations:

$$
\begin{array}{ll}
\pi\left(\left\{\alpha_{2}\right\}, \delta_{+},\left(t_{3}, 1\right)\right)=\operatorname{Ind}_{\mathcal{H}^{P}}^{\mathcal{H}}\left(\delta_{+} \circ \phi_{\left(t_{3}, 1\right)}\right) & q_{0} q_{2} \neq 1, t_{3} \in S^{1}, \\
\pi\left(\left\{\alpha_{2}\right\}, \delta_{-},\left(t_{3}, 1\right)\right)=\operatorname{Ind}_{\mathcal{H}^{P}}^{\mathcal{H}}\left(\delta_{-} \circ \phi_{\left(t_{3}, 1\right)}\right) & q_{0} / q_{2} \neq 1, t_{3} \in S^{1} .
\end{array}
$$

The group $\mathcal{G}_{P}$ acts on these families by $s_{3}\left(t_{3}, 1\right)=\left(t_{3}^{-1}, 1\right)$. A fundamental domain is, for both families, given by $t \in\left\{e^{i \phi}: \phi \in[0, \pi]\right\}$. For $\phi \in(0, \pi)$ the representations $\pi\left(\left\{\alpha_{2}\right\}, \delta_{+},\left(e^{i \phi}, 1\right)\right)$ are irreducible, because the isotropy group in $\mathcal{G}_{P}$ is trivial. For $\phi \in\{0, \pi\}$ the intertwining operator associated to $s_{3} \in \mathcal{G}_{P}$ is not necessarily scalar, so we find either one or two irreducible constituents. Remarkably 
enough, this depends not only on the parameters $q_{0}$ and $q_{2}$ of $\mathcal{H}_{P}$, but also on $q_{1}$, as we will see later.

$$
P=\left\{\alpha_{2}\right\}=F_{0}
$$

Here simply $\mathcal{H}^{P}=\mathcal{H}_{P}=\mathcal{H}(\mathcal{R}, q)$. We have to determine all residual points, and how many inequivalent discrete series they carry. The former can be done by hand, but that is quite elaborate. It is more convenient to use Opd2, Theorem 7.7] and HeOp Proposition 4.2], which say that for generic $q$ there are 40 residual points. Representatives for the $5 W_{0}$-orbits are:

$$
\begin{aligned}
& r_{1}(q)=\left(q_{0}^{1 / 2} q_{2}^{1 / 2} q_{1}^{-1}, q_{0}^{1 / 2} q_{2}^{1 / 2}\right), \\
& r_{2}(q)=\left(q_{0}^{-1 / 2} q_{2}^{-1 / 2} q_{1}^{-1}, q_{0}^{-1 / 2} q_{2}^{-1 / 2}\right), \\
& r_{3}(q)=\left(-q_{0}^{1 / 2} q_{2}^{-1 / 2} q_{1}^{-1},-q_{0}^{1 / 2} q_{2}^{-1 / 2}\right), \\
& r_{4}(q)=\left(-q_{0}^{-1 / 2} q_{2}^{1 / 2} q_{1}^{-1},-q_{0}^{1 / 2} q_{2}^{-1 / 2}\right), \\
& r_{5}(q)=\left(-q_{0}^{1 / 2} q_{2}^{-1 / 2}, q_{0}^{-1 / 2} q_{2}^{-1 / 2}\right) .
\end{aligned}
$$

Since every $W_{0}$-orbit of residual points carries at least one discrete series representation,

$$
\operatorname{dim}_{\mathbb{Q}}\left(G_{\mathbb{Q}}^{0}(\mathcal{H}(\mathcal{R}, q)) / G_{\mathbb{Q}}^{1}(\mathcal{H}(\mathcal{R}, q))\right)=\operatorname{dim}_{\mathbb{Q}}\left(G_{\mathbb{Q}}^{0}(\mathcal{S}(\mathcal{R}, q)) / G_{\mathbb{Q}}^{1}(\mathcal{S}(\mathcal{R}, q))\right) \geq 5 .
$$

On the other hand one can easily check, for example, with the calculations for $P=\emptyset, q=1$, that $\operatorname{dim}_{\mathbb{Q}}\left(G_{\mathbb{Q}}^{0}(W) / G_{\mathbb{Q}}^{1}(W)\right)=5$. With 3.38) we deduce that every $W r_{i}(q)$ carries a unique discrete series representation $\delta\left(r_{i}\right)$, so far for generic parameter functions.

For nongeneric $q$ the $W r_{i}(q)$ are still the only possible residual points, but some of them may cease to be residual for certain $q$. In such cases $r_{i}(q)$ is absorbed by the tempered part $r T_{u n}^{P}$ of some one-dimensional residual coset $r T^{P}$. (If $r_{i}(q)$ is absorbed by $T_{u n}$, which is the tempered part of the two-dimensional residual coset $T$, then $r_{i}(q)$ is also absorbed by a one-dimensional residual coset.) This happens in the following cases:

\begin{tabular}{lll} 
residual point & $q$ & absorbed by \\
\hline$r_{1}(q)$ & $q_{0} q_{2}=q_{1}$ & $W_{0}\left(q_{1}^{1 / 2}, q_{1}^{-1 / 2}\right) T_{u n}^{\alpha_{1}}$ \\
& $q_{0} q_{2}=q_{1}^{2}$ & $W_{0}\left(1, q_{0}^{1 / 2} q_{2}^{1 / 2}\right) T_{u n}^{\alpha_{2}}$ \\
$r_{2}(q)$ & $q_{0} q_{2}=q_{1}^{-1}$ & $W_{0}\left(q_{1}^{1 / 2}, q_{1}^{-1 / 2}\right) T_{u n}^{\alpha_{1}}$ \\
& $q_{0} q_{2}=q_{1}^{-2}$ & $W_{0}\left(1, q_{0}^{1 / 2} q_{2}^{1 / 2}\right) T_{u n}^{\alpha_{2}}$ \\
$r_{3}(q)$ & $q_{0} / q_{2}=q_{1}$ & $W_{0}\left(q_{1}^{1 / 2}, q_{1}^{-1 / 2}\right) T_{u n}^{\alpha_{1}}$ \\
& $q_{0} / q_{2}=q_{1}^{2}$ & $W_{0}\left(1,-q_{0}^{1 / 2} q_{2}^{-1 / 2}\right) T_{u n}^{\alpha_{2}}$ \\
$r_{4}(q)$ & $q_{0} / q_{2}=q_{1}^{-1}$ & $W_{0}\left(q_{1}^{1 / 2}, q_{1}^{-1 / 2}\right) T_{u n}^{\alpha_{1}}$ \\
& $q_{0} / q_{2}=q_{1}^{-2}$ & $W_{0}\left(1,-q_{0}^{1 / 2} q_{2}^{-1 / 2}\right) T_{u n}^{\alpha_{2}}$ \\
$r_{5}(q)$ & $q_{0}=q_{2}$ & $W_{0}\left(1, q_{0}^{1 / 2} q_{2}^{1 / 2}\right) T_{u n}^{\alpha_{2}}$ \\
& $q_{0} q_{2}=1$ & $W_{0}\left(1,-q_{0}^{1 / 2} q_{2}^{-1 / 2}\right) T_{u n}^{\alpha_{2}}$
\end{tabular}

It is also possible that two orbits of residual points confluence, but stay residual. The deep result OpSo2, Theorem 3.4] says that in general situations of this type the discrete series representations with confluencing central character do not merge and remain irreducible. 
The geometric content of the Aubert-Baum-Plymen conjecture is best illustrated with some pictures of the tempered dual of $\mathcal{H}(\mathcal{R}, q)$, for various $q$. Of course $T$ has real dimension four, so we cannot draw it. But the unitary principal series can be parametrized by $T_{u n} / W_{0}$, which is simply a $45-45-90$ triangle.

The other components of $\operatorname{Irr}(\mathcal{S}(\mathcal{R}, q))$ will lie close to $T_{u n} / W_{0}$ if $q$ is close to 1 , which we will assume in our pictures. We indicate what confluence occurs when $q$ is scaled to 1 by drawing any $\pi \in \operatorname{Irr}(\mathcal{S}(\mathcal{R}, q))$ close to the unitary part of its central character. To distinguish the three one-dimensional components, we denote the series obtained from inducing $\delta_{1} / \delta_{+} / \delta_{-}$by $L_{1} / L_{+} / L_{-}$.

Finally, we have to represent graphically how many inequivalent irreducible representations a given parabolically induced representation $\pi(\xi)$ contains. By default, $\pi(\xi)$ is itself irreducible. When $\pi(\xi)$ contains two different irreducibles, we draw the corresponding point fatter. When there are more than two, we write the number of irreducibles next to it.

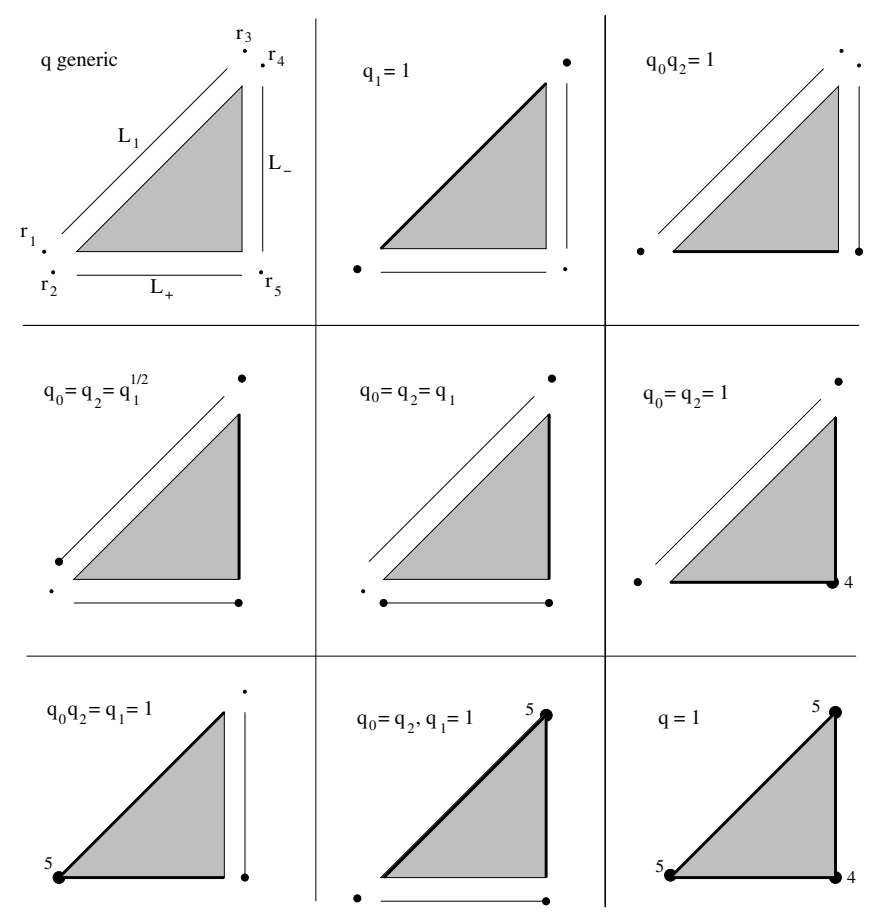

Figure 5.1. The tempered dual for various values of $q$

Almost everything in Figure 5.1 can be deduced from the above calculations, the ABP-conjecture (or rather Theorem 5.4.2) and OpSo2, Theorem 3.4]. The only thing that cannot be detected with these methods is what happens at the confluences $r_{1}(q) \rightarrow\left(q_{1}^{1 / 2}, q_{1}^{-1 / 2}\right) \in L_{1}$ for $q_{0}=q_{2}=q_{1}^{1 / 2}$ and $r_{1}(q) \rightarrow\left(1, q_{2}\right) \in L_{+}$for $q_{0}=q_{1}=q_{2}$. For these $q$ there are four unitary induction data that give rise to representations with central character in $T_{r s} / W_{0}$. So three of them are irreducible, and one contains two different irreducibles. We can see that the reducibility does not occur in the unitary principal series or in the discrete series, which leaves the 
two intermediate series. Fortunately, one can explicitly determine all subrepresentations of $\pi\left(\left\{\alpha_{1}\right\}, \delta_{1},(1,1)\right)$ (for $q_{0}=q_{2}=q_{1}^{1 / 2}$ ) and of $\pi\left(\left\{\alpha_{2}\right\}, \delta_{+},(1,1)\right)$ (for $\left.q_{0}=q_{1}=q_{2}\right)$; see Slo1, Section 8.1.2]. In fact, the graded Hecke algebras corresponding to these parameter functions are precisely the ones associated to $S p_{4}$ and to $\mathrm{SO}_{5}$. Hence one may also determine the reducibility of the aforementioned representations via the Deligne-Langlands-Kazhdan-Lusztig parametrization. Finally, it is possible to analyse these parabolically induced representations with R-groups, as in DeOp2.

The comparison of the tempered duals for different parameter functions clearly shows that this affine Hecke algebra behaves well with respect to general parameter deformations, not necessarily of the form $q \mapsto q^{\epsilon}$. We see that for small pertubations of $q$ it is always possible to find regions $U / W_{0} \subset T / W_{0}$ such that the number of tempered irreducibles with central character in $U / W_{0}$ remains stable. The $W_{0^{-}}$ types of these representations can change, however. It is reasonable to expect that something similar holds for general affine Hecke algebras, probably that would follow from the existence of an appropriate asymptotic Hecke algebra [Lus8].

\section{ACKNOWLEDGEMENTS}

The author learned a lot about affine Hecke algebras from Eric Opdam, first as a $\mathrm{PhD}$ student and later as co-author. Without that support, this article would not have been possible. The author also thanks Roger Plymen for providing background information about several conjectures, and Anne-Marie Aubert for many detailed comments, in particular, concerning Section 1.6. Finally, two anonymous referees read the paper very carefully and made many useful suggestions for improvement.

\section{REFERENCES}

[ABP1] A.-M. Aubert, P.F. Baum, R.J. Plymen, "The Hecke algebra of a reductive $p$-adic group: a view from noncommutative geometry", pp. 1-34 in: Noncommutative geometry and number theory, Aspects of Mathematics E37, Vieweg Verlag, 2006

[ABP2] A.-M. Aubert, P.F. Baum, R.J. Plymen, "Geometric structure in the representation theory of $p$-adic groups", C.R. Acad. Sci. Paris Ser. I 345 (2007), 573-578 MR.2374467 (2009b:20086)

[ABP3] A.-M. Aubert, P.F. Baum, R.J. Plymen, "Geometric structure in the principal series of the p-adic group $G_{2}$ ", Representation Theory 15 (2011), 126-169 MR.2772586

[ABP4] A.-M. Aubert, P.F. Baum, R.J. Plymen, "Geometric structure in the representation theory of $p$-adic groups II", preprint, 2010

[BaJa1] D. Ban, C. Jantzen, "The Langlands classification for non-connected $p$-adic groups", Israel J. of Math. 126 (2001), 239-261 MR1882038(2002i:22018)

[BaJa2] D. Ban, C. Jantzen, "The Langlands classification for non-connected $p$-adic groups II. Multiplicity one", Proc. Amer. Math. Soc. 131 (2003), 3297-3304 MR.1992872 (2004e:22019)

[BaCi] D. Barbasch, D. Ciubotaru, "Unitary functorial correspondences for $p$-adic groups", arxiv:0909.5241, 2010

[BaMo1] D. Barbasch, A. Moy, "A unitarity criterion for p-adic groups", Inv. Math. 98 (1989), 19-37 MR.1010153 (90m:22038)

[BaMo2] D. Barbasch, A. Moy, "Reduction to real infinitesimal character in affine Hecke algebras", J. Amer. Math. Soc. 6.3 (1993), 611-635 MR.1186959 (93k:22015) 
[BCH] P.F. Baum, A. Connes, N. Higson, "Classifying space for proper actions and K-theory of group $C^{*}$-algebras", pp. 240-291 in: $C^{*}$-algebras: 1943-1993. A fifty year celebration, Contemp. Math. 167, American Mathematical Society, 1994 MR.1292018 (96c:46070)

[BHP] P.F. Baum, N. Higson, R.J. Plymen, "Representation theory of $p$-adic groups: a view from operator algebras", Proc. Sympos. Pure. Math. 68 (2000), 111-149 MR.1767895 (2002k:22008)

[BaNi] P.F. Baum, V. Nistor, "Periodic cyclic homology of Iwahori-Hecke algebras", K-Theory 27.4 (2002), 329-357 MR,1962907 (2004b:16011)

[BeDe] J.N. Bernstein, P. Deligne, "Le "centre" de Bernstein", pp. 1-32 in: Représentations des groupes réductifs sur un corps local, Travaux en cours, Hermann, 1984 MR771671 (86e:22028)

[BeRu] J. Bernstein, K.E. Rumelhart, "Representations of p-adic groups", draft, 1993

[Bla] B. Blackadar, $K$-theory for operator algebras 2nd ed., Mathematical Sciences Research Institute Publications 5, Cambridge University Press, 1998 MR.1656031 (99g:46104)

[Blo] C. Blondel, "Propagation de paires couvrantes dans les groupes symplectiques", Representation Theory 10 (2006), 399-434 MR.2266698 (2007m:22012)

[Bor] A. Borel, "Admissible representations of a semi-simple group over a local field with vectors fixed under an Iwahori subgroup", Inv. Math. 35 (1976), 233-259 MR0444849 (56:3196)

[Bos] J.-B. Bost, "Principe d'Oka, K-théorie et systèmes dynamiques non commutatifs", Invent. Math. 101 (1990), 261-333 MR.1062964 (92j:46126)

[BuKu1] C.J. Bushnell, P.C. Kutzko, The admissible dual of $G L(N)$ via compact open subgroups, Annals of Mathematics Studies 129, Princeton University Press, 1993 MR1204652 (94h:22007)

[BuKu2] C.J. Bushnell, P.C. Kutzko, "Smooth representations of reductive $p$-adic groups: structure theory via types", Proc. London Math. Soc. 77.3 (1998), 582-634 MR.1643417 (2000c:22014)

[BuKu3] C.J. Bushnell, P.C. Kutzko, "Semisimple types in $G L_{n}$ ", Compositio Math. 119.1 (1999), 53-97 MR1711578 (2000i:20072)

[Cas] W. Casselman, "Introduction to the theory of admissible representations of $p$-adic reductive groups", draft, 1995

[ChGi] N. Chriss, V. Ginzburg, Representation theory and complex geometry, Birkhäuser, 1997 MR:1433132 (98i:22021)

[Ciu] D. Ciubotaru, "On unitary unipotent representations of $p$-adic groups and affine Hecke algebras with unequal parameters", Representation Theory 12 (2008), 453-498 MR2465803 (2010f:22015)

[CiKa] D. Ciubotaru, S. Kato, "Tempered modules in the exotic Deligne-Langlands classification", Adv. Math. 226.2 (2011), 1538-1590 MR2737793

[CiTr] D. Ciubotaru, P.E. Trapa, "Functors for unitary representations of classical real groups and affine Hecke algebras", Adv. Math. 227.4 (2011), 1585-1611. MR.2799806

[CuRe] C.W. Curtis, I. Reiner, Representation theory of finite groups and associative algebras, Pure and Applied Mathematics 11, John Wiley \& Sons, 1962 MR1013113 (90g:16001)

[DeOp1] P. Delorme, E.M. Opdam, "The Schwartz algebra of an affine Hecke algebra" J. Reine Angew. Math. 625 (2008), 59-114 MR2482216 (2010a:22013)

[DeOp2] P. Delorme, E.M. Opdam, "Analytic R-groups of affine Hecke algebras", arXiv:0909.1227, to appear in J. Reine Angew. Math.

[Eve] S. Evens, "The Langlands classification for graded Hecke algebras", Proc. Amer. Math. Soc. 124.4 (1996), 1285-1290 MR.1322921 (96g:22022)

[GoRo] D. Goldberg, A. Roche, "Hecke algebras and $S L_{n}$-types", Proc. London Math. Soc. 90.1 (2005), 87-131 MR2107039 (2005i:22017)

[HeOp] G.J. Heckman, E.M. Opdam. "Yang's system of particles and Hecke algebras", Ann. of Math. 145.1 (1997), 139-173 MR:1432038(99g:33052a)

[Hei] V. Heiermann, "Opérateurs d'entrelacement et algèbres de Hecke avec paramètres d'un groupe réductif $p$-adique - le cas des groupes classiques", Selecta Math. 17 (2011), DOI: 10.1007/s00029-011-0056-0

[Hum] J.E. Humphreys, Reflection groups and Coxeter groups, Cambridge Studies in Advanced Mathematics 29, Cambridge University Press, 1990 MR.1066460 (92h:20002) 
[IwMa] N. Iwahori, H. Matsumoto, "On some Bruhat decomposition and the structure of the Hecke rings of the $p$-adic Chevalley groups", Inst. Hautes Études Sci. Publ. Math 25 (1965), 5-48 MR0185016 (32:2486)

[Kat1] S.-I. Kato, "Irreducibility of principal series representations for Hecke algebras of affine type", J. Fac. Sci. Univ. Tokyo Sect. IA Math. 28.3 (1981), 929-943 MR656065 (84b:22029)

[Kat2] Syu Kato, "An exotic Deligne-Langlands correspondence for symplectic groups", Duke Math. J. 148.2 (2009), 305-371 MR2524498 (2010k:20013)

[KaLu1] D. Kazhdan, G. Lusztig, "Equivariant $K$-theory and representations of Hecke algebras II", Invent. Math. 80.2 (1985), 209-231. MR788408 (88f:22054b)

[KaLu2] D. Kazhdan, G. Lusztig, "Proof of the Deligne-Langlands conjecture for Hecke algebras", Invent. Math. 87 (1987), 153-215 MR862716 (88d:11121)

[Kim1] Ju-Lee Kim, "Hecke algebras of classical groups over $p$-adic fields and supercuspidal representations", Amer. J. Math. 121.5 (1999), 967-1029 MR:1713299 (2001g:11075)

[Kim2] Ju-Lee Kim, "Hecke algebras of classical groups over $p$-adic fields II" Compositio Math. 127.2 (2001), 117-167 MR1845898 (2002g:22034)

[Kon] T. Konno, "A note on the Langlands classification and irreducibility of induced representations of $p$-adic groups" Kyushu J. Math. 57 (2003), 383-409 MR:2050093 (2005b:22020)

[KrRa] C. Kriloff, A. Ram, "Representations of graded Hecke algebras", Representation Theory 6 (2002), 31-69 MR.1915086 (2003h:20013)

[Laf] V. Lafforgue, "K-théorie bivariante pour les algèbres de Banach et conjecture de BaumConnes", Invent. Math. 149.1 (2002), 1-95 MR1914617 (2003d:19008)

[Lan] R.P. Langlands, "On the classification of irreducible representations of real algebraic groups", pp. 101-170 in: Representation theory and harmonic analysis on semisimple Lie groups, Math. Surveys Monogr. 31, American Mathematical Society, 1989 MR.1011897|(91e:22017)

[Lus1] G. Lusztig, Representations of finite Chevalley groups, CBMS Regional Conference Series in Mathematics 39, American Mathematical Society, 1978 MR518617 (80f:20045)

[Lus2] G. Lusztig, "Equivariant K-theory and representations of Hecke algebras", Proc. Amer. Math. Soc. 94.2 (1985), 337-342 MR784189 (88f:22054a)

[Lus3] G. Lusztig, "Cells in affine Weyl groups II", J. Algebra 109 (1987), 536-548 MR.902967 $(88 \mathrm{~m}: 20103 \mathrm{a})$

[Lus4] G. Lusztig, "Cells in affine Weyl groups III", J. Fac. Sci. Univ. Tokyo 34.2 (1987), 223-243 MR914020 (88m:20103b)

[Lus5] G. Lusztig, "Representations of affine Hecke algebras", Astérisque 171-172 (1989), 73-84 MR $1021500(90 \mathrm{k}: 22028)$

[Lus6] G. Lusztig, "Affine Hecke algebras and their graded version", J. Amer. Math. Soc 2.3 (1989), 599-635 MR991016 (90e:16049)

[Lus7] G. Lusztig, "Classification of unipotent representations of simple p-adic groups", Int. Math. Res. Notices 11 (1995), 517-589 MR.1369407 (98b:22034)

[Lus8] G. Lusztig, Hecke algebras with unequal parameters, CRM Monograph Series 18, American Mathematical Society, 2003 MR.1974442 (2004k:20011)

[Lus9] G. Lusztig, "From conjugacy classes in the Weyl group to unipotent classes", arXiv:1003.0412, 2010

[Mor] L. Morris, "Tamely ramified intertwining algebras", Invent. Math. 114.1 (1993), 1-54 MR 1235019 (94g:22035)

[Moy1] A. Moy, "Representations of $G S p(4)$ over a $p$-adic field. I and II.", Compositio Math. 66.3 (1988), 237-284 and 285-328 MR948308 (90d:22022)

[Moy2] A. Moy, "Representations of $U(2,1)$ over a $p$-adic field", J. Reine Angew. Math. 372 (1986), 178-208 MR863523 (88a:22031)

[MoPr] A. Moy, G. Prasad, "Jacquet functors and unrefined minimal K-types", Comment. Math. Helvetici 71 (1996), 98-121 MR.1371680 (97c:22021)

[Nis] V. Nistor, "A non-commutative geometry approach to the representation theory of reductive $p$-adic groups: Homology of Hecke algebras, a survey and some new results", pp. 301-323 in: Noncommutative geometry and number theory, Aspects of Mathematics E37, Vieweg Verlag, 2006 MR2327310(2008f:22010) 
[Opd1] E.M. Opdam, "A generating function for the trace of the Iwahori-Hecke algebra", Progr. Math. 210 (2003), 301-323 MR.1985730 (2004f:20013)

[Opd2] E.M. Opdam, "On the spectral decomposition of affine Hecke algebras", J. Inst. Math. Jussieu 3.4 (2004), 531-648 MR2094450(2005i:20008)

[Opd3] E.M. Opdam, "Hecke algebras and harmonic analysis", pp. 1227-1259 of: Proceedings ICM Madrid 2006 Vol. II, European Mathematical Society Publishing House, 2006 MR2275643(2008j:22006)

[Opd4] E.M. Opdam, "The central support of the Plancherel measure of the Hecke algebra", Moscow Math. J. 7 (2007), 723-741 MR.2372211(2009e:22006)

[OpSo1] E.M. Opdam, M. Solleveld, "Homological algebra for affine Hecke algebras", Advances in Mathematics 220 (2009), 1549-1601 MR2493620 (2010h:20014)

[OpSo2] E.M. Opdam, M. Solleveld, "Discrete series characters for affine Hecke algebras and their formal dimensions", Acta Mathematica 205 (2010), 105-187 MR2736154

[Phi] N.C. Phillips, " $K$-theory for Fréchet algebras". Int. J. Math. 2.1 (1991), 77-129 MR.1082838 (92e:46143)

[Ply1] R.J. Plymen, "Noncommutative geometry: illustrations from the representation theory of GL(n)", lecture notes, 1993, http://eprints.ma.man.ac.uk/1346

[Ply2] R.J. Plymen, "Reduced $C^{*}$-algebra for reductive $p$-adic groups", J. Funct. Anal. 88.2 (1990), 251-266 MR1038441(91a:46064)

[RaRa] A. Ram, J. Ramagge, "Affine Hecke algebras, cyclotomic Hecke algebras and Clifford theory", pp. 428-466 in: A tribute to C.S. Seshadri (Chennai 2002), Trends in Mathematics, Birkhäuser, 2003 MR2017596 (2004i:20009)

[Ree1] M. Reeder "Nonstandard intertwining operators and the structure of unramified principal series representations", Forum Math. 9 (1997), 457-516 MR.1457135 (98j:22028)

[Ree2] M. Reeder, "Isogenies of Hecke algebras and a Langlands correspondence for ramified principal series representations", Representation Theory 6 (2002), 101-126 MR1915088 (2003j:22021)

[Roc1] A. Roche, "Types and Hecke algebras for principal series representations of split reductive $p$-adic groups", Ann. Sci. École Norm. Sup. 31.3 (1998), 361-413 MR.1621409 (99d:22028)

[Roc2] A. Roche, "The Bernstein decomposition and the Bernstein centre", pp. 3-53 in: Ottawa lectures on admissible representations of reductive p-adic groups, Fields Inst. Monogr. 26, American Mathetical Society, 2009 MR2508719 (2010c:22022)

[Rog] J. Rogawski "On modules over the Hecke algebra of a $p$-adic group", Inv. Math. 79 (1985), 225-249 MR782228 (86j:22028)

[Ros] J.M. Rosenberg, "Appendix to: "Crossed products of UHF algebras by product type actions" by O. Bratteli", Duke Math. J. 46.1 (1979), 25-26 MR.523599 (82a:46064)

[Slo1] K. Slooten, A combinatorial generalization of the Springer correspondence for classical type, PhD Thesis, Universiteit van Amsterdam, 2003

[Slo2] K. Slooten, "Generalized Springer correspondence and Green functions for type B/C graded Hecke algebras", Adv. Math. 203 (2005), 34-108 MR.2231067 (2007k:20015)

[Sol1] M. Solleveld, "Some Fréchet algebras for which the Chern character is an isomorphism", K-Theory 36 (2005), 275-290 MR2275008 (2007k:19005)

[Sol2] M. Solleveld, "Periodic cyclic homology of Hecke algebras and their Schwartz completions", arXiv:math.KT/0605042, 2006

[Sol3] M. Solleveld, Periodic cyclic homology of affine Hecke algebras, PhD Thesis, Universiteit van Amsterdam, 2007, arXiv:0910.1606

[Sol4] M. Solleveld, "Periodic cyclic homology of reductive $p$-adic groups", Journal of Noncommutative Geometry 3.4 (2009), 501-558 MR.2539769 (2010j:19007)

[Sol5] M. Solleveld, "Parabolically induced representations of graded Hecke algebras", Algebras and Representation Theory (2010), DOI: 10.1007/s10468-010-9240-8

[Sol6] M. Solleveld, "Homology of graded Hecke algebras" J. Algebra 323 (2010), 1622-1648 MR2588128 (2011d:20008)

[Spr] T.A. Springer, "A construction of representations of Weyl groups", Invent. Math. 44.3 (1978), 279-293 MR0491988(58:11154) 
[Wal] J.-L. Waldspurger, "La formule de Plancherel pour les groupes p-adiques (d'après Harish-Chandra)", J. Inst. Math. Jussieu 2.2 (2003), 235-333 MR.1989693 (2004d:22009)

[Xi] N. Xi, Representations of affine Hecke algebras, Lecture Notes in Mathematics 1587, Springer-Verlag, 1994 MR,1320509 (96i:20058)

Mathematisches Institut, Georg-August-Universität Göttingen, Bunsenstrasse 3-5, 37073 Göttingen, Germany

E-mail address: Maarten.Solleveld@mathematik.uni-goettingen.de 